NUREG/CR-6041

BNL-NUREG-52375

\title{
Disposal Unit Source Term (DUST) Data Input Guide
}

Manuscript Completed: April 1993

Date Published: May 1993

Prepared by

T. M. Sullivan

Brookhaven National Laboratory

Upton, NY 11973

Prepared for

Division of Low-Level Waste Management and Decommissioning

Office of Nuclear Material Safety and Safeguards

U.S. Nuclear Regulatory Commission

Washington, DC 20555

NRC FIN L1409 


\begin{abstract}
Performance assessment of a low-level waste (LLW) disposal facility begins with an estimation of the rate at which radionuclides migrate out of the facility (i.e., the source term). The focus of this work is to develop a methodology for calculating the source term. This work helps support activities to develop further guidance in the performance assessment area. In general, the source term is influenced by the radionuclide inventory, the wasteforms and containers used to dispose of the inventory, and the physical processes that lead to release from the facility (fluid flow, container degradation, wasteform leaching, and radionuclide transport). Many of these physical processes are influenced by the design of the disposal facility (e.g., infiltration of water). The complexity of the problem and the absence of appropriate data prevent development of an entirely mechanistic representation of radionuclide release from a disposal facility. Typically, a number of assumptions, based on knowledge of the disposal system, are used to simplify the problem. This has been done and the resulting models have been incorporated into the computer code DUST (Disposal Unit Source Term). This document presents the models used to calculate release from a disposal facility, verification of the model, and instructions on the use of the DUST code. In addition to DUST, a preprocessor, DUSTIN, which helps the code user create input decks for DUST and a post-processor, GRAFXT, which takes selected output files and plots them on the computer terminal have been written. Use of these codes is also described. In using DUST, as with all computer models, the validity of the predictions relies heavily on the validity of the input parameters. Often, the largest uncertainties arise from uncertainty in the input parameters. Therefore, it is crucial to document and support the use of these parameters. The DUST code, because of its flexibility and ability to compute release rates quickly, will be extremely useful for screening to determine the radionuclide released at the highest rate, parameter sensitivity analysis and, with proper choice of the input parameters, provide upper bounds to release rates.
\end{abstract}


ABSTRACT $\ldots \ldots \ldots \ldots \ldots \ldots \ldots \ldots \ldots \ldots \ldots \ldots \ldots \ldots \ldots \ldots \ldots$ iii EXECUTIVE SUMMARY $\ldots \ldots \ldots \ldots \ldots \ldots \ldots \ldots \ldots \ldots \ldots \ldots \ldots \ldots \ldots$ xv

1. INTRODUCTION $\ldots \ldots \ldots \ldots \ldots \ldots \ldots \ldots \ldots \ldots \ldots \ldots \ldots \ldots \ldots \ldots \ldots \ldots \ldots$

2. MODEL SELECTION AND GOVERNING EQUATIONS $\ldots \ldots \ldots \ldots \ldots \ldots .2-1$

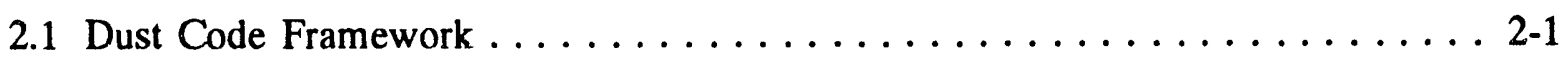

2.2 Radionuclide Transport $\ldots \ldots \ldots \ldots \ldots \ldots \ldots \ldots \ldots \ldots \ldots \ldots \ldots \ldots \ldots \ldots \ldots .2$

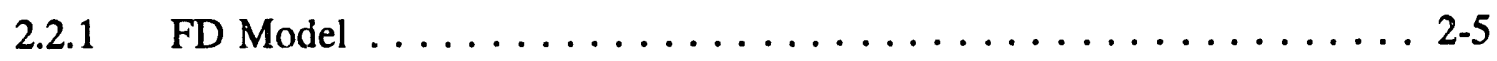

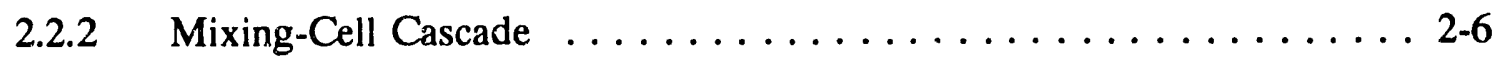

2.3 Fluid Flow $\ldots \ldots \ldots \ldots \ldots \ldots \ldots \ldots \ldots \ldots \ldots \ldots \ldots \ldots \ldots \ldots \ldots \ldots$

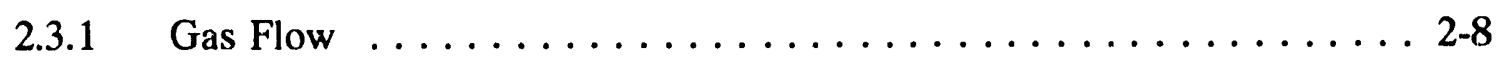

2.4 Container Degradation $\ldots \ldots \ldots \ldots \ldots \ldots \ldots \ldots \ldots \ldots \ldots \ldots \ldots \ldots \ldots$

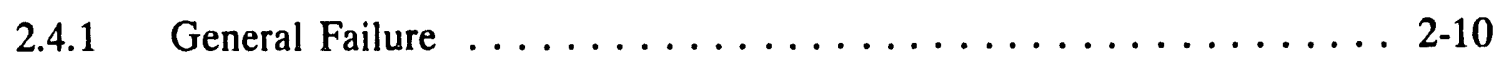

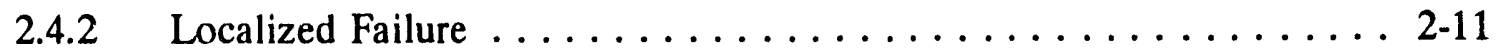

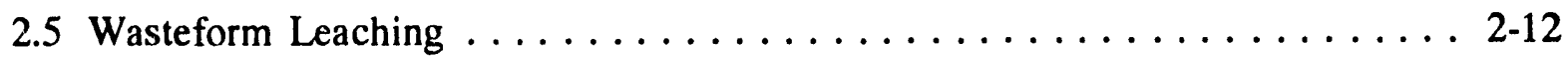

2.5.1 Solubility-limited Release $\ldots \ldots \ldots \ldots \ldots \ldots \ldots \ldots \ldots \ldots . \ldots \ldots$

2.5.2 Surface Rinse with Partitioning $\ldots \ldots \ldots \ldots \ldots \ldots \ldots \ldots \ldots \ldots \ldots \ldots$

2.5.2.1 MCMC Model . . . . . . . . . . . . . . 2-14

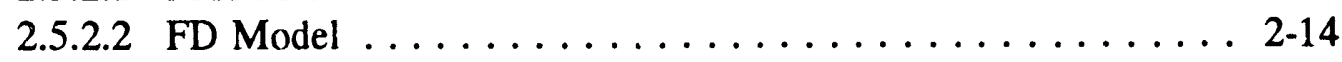

2.5.3 Diffusion Release Model . . . . . . . . . . . . . . . . . 2-16

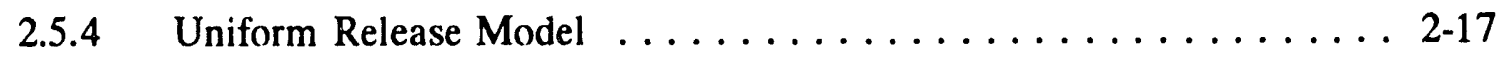

2.5.5 Influence of Localized Failure on Release . . . . . . . . . 2-18

2.5.6 Selection of Release Models $\ldots \ldots \ldots \ldots \ldots \ldots \ldots \ldots \ldots .2-19$

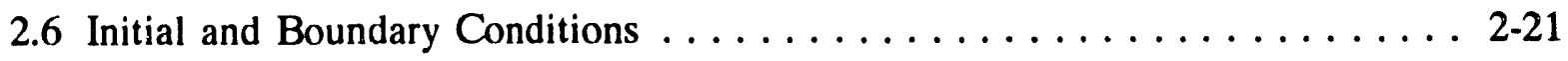




\section{TABLE OF CONTENTS}

(continued)

$\underline{\text { PAGE }}$

3. PROCEDURE FOR CALCULATING RELEASES USING DUST $\ldots \ldots \ldots \ldots$ 3-1

4. APPLICABILITY AND LIMITATIONS OF THE DUST CODE $\ldots \ldots \ldots \ldots \ldots$. . .

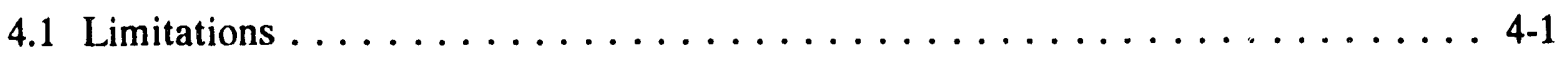

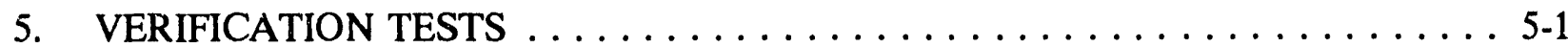

6. DUSTIN: A PREPROCESSOR THAT CREATES AN INPUT DECK FOR DUST . 6-1

6.1 General Problem Definitions $\ldots \ldots \ldots \ldots \ldots \ldots \ldots \ldots \ldots \ldots .7$

6.1.1 Problem Title $\ldots \ldots \ldots \ldots \ldots \ldots \ldots \ldots \ldots \ldots \ldots \ldots .6 \ldots \ldots$

5.1 .2 Radionuclide ........................6.7 6-7

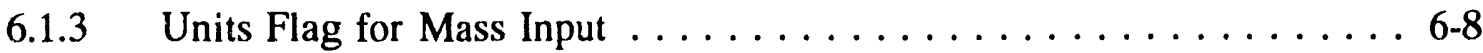

6.1 .4 Number of Computational Cells . . . . . . . . . . . . . . . 6-8

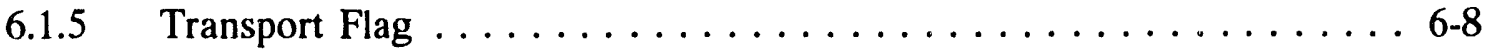

6.2 Time Parameters $\ldots \ldots \ldots \ldots \ldots \ldots \ldots \ldots \ldots \ldots \ldots \ldots \ldots .6 \ldots \ldots$

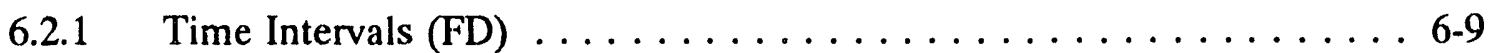

6.2.2 Number of Output (MCMC) or Time Step Changes (FD) . . . . . . . 6-9

6.2.3 Output Times or Time Step Changes $\ldots \ldots \ldots \ldots \ldots \ldots \ldots \ldots .6 .9$

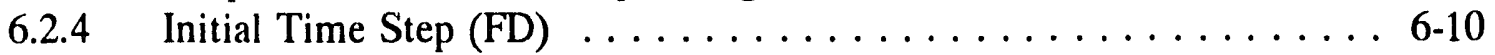

$6.2 .5 \quad$ Fractional Time Step (FD) $\ldots \ldots \ldots \ldots \ldots \ldots \ldots \ldots \ldots$ 6-10

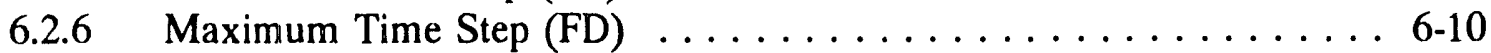

6.2 .7 Maximum Simulation Time (FD) $\ldots \ldots \ldots \ldots \ldots \ldots \ldots \ldots .6 \ldots \ldots$

6.3 Material Assignments/Properties . . . . . . . . . . . . . . 6-11

6.3.1 Number of Different Materials . . . . . . . . . . . . . 6-12

6.3.2 Material Type Assignments . . . . . . . . . . . . . . . 6-12

6.3.3 Material Properties ...................... 6-14 


\section{TABLE OF CONTENTS}

(continued)

$\underline{\text { PAGE }}$

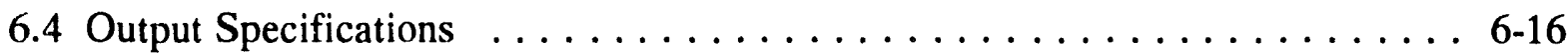

6.4.1 Printer Control Parameters $\ldots \ldots \ldots \ldots \ldots \ldots \ldots \ldots \ldots . \ldots .6 .16$

6.4.2 Number of Concentration Traces $\ldots \ldots \ldots \ldots \ldots \ldots \ldots \ldots .6 .18$

6.4.3 Location of Concentration Traces . . . . . . . . . . . . . 6-18

6.4 .4 Number of Flux Traces . . . . . . . . . . . . . . . . 6-19

6.4 .5 Location of Flux Traces ..................... 6-19

6.4.6 Number of Time Steps Between Traces $\ldots \ldots \ldots \ldots \ldots \ldots .6$ 6-19

6.5 Facility Dimensions and Coordinates $\ldots \ldots \ldots \ldots \ldots \ldots \ldots \ldots \ldots 6-20$

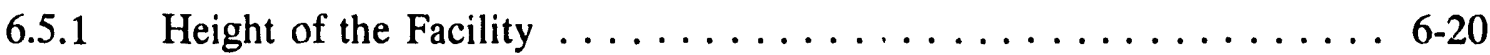

6.5.2 Cross Sectional Area . . . . . . . . . . . . . . . . . 6-20

6.5.3 Node Coordinates (FD Model) . . . . . . . . . . . . 6-20

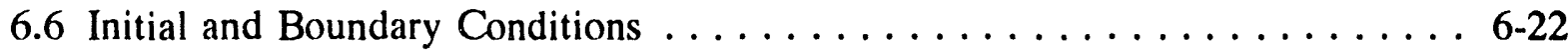

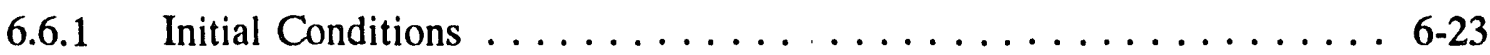

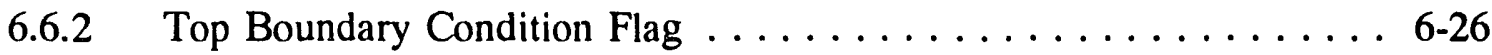

6.6.3 Bottom Boundary Condition Flag . . . . . . . . . . . . 6-26

6.6.4 Number of Boundary Condition Data Points . . . . . . . . . . 6-26

6.6.5 Boundary Condition Table for the Top Boundary . . . . . . . . 6-26

6.6.6 Boundary Condition Table for the Bottom Boundary ........ 6-27

6.7 Water Flow and Moisture Content $\ldots \ldots \ldots \ldots \ldots \ldots \ldots \ldots \ldots .6 .27$

6.7.1 Darcy Velocity . . . . . . . . . . . . . . . . . 6

6.7.2 Moisture Content Values . . . . . . . . . . . . . . . 6 6-29

6.8 Container Parameters $\ldots \ldots \ldots \ldots \ldots \ldots \ldots \ldots \ldots \ldots \ldots \ldots .32$

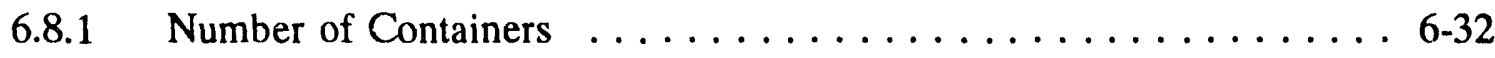

6.8.2 Time of General Failure $\ldots \ldots \ldots \ldots \ldots \ldots \ldots \ldots \ldots \ldots .6 \ldots \ldots .6 \ldots$

6.8.3 Location of the Containers $\ldots \ldots \ldots \ldots \ldots \ldots \ldots \ldots \ldots .6 \ldots \ldots$

6.8 .4 Number of Failure Types (FD) $\ldots \ldots \ldots \ldots \ldots \ldots \ldots \ldots .35$ 


\section{TABLE OF CONTENTS \\ (continued)}

$\underline{\text { PAGE }}$

6.8 .5 Corrosion Model Flag $\ldots \ldots \ldots \ldots \ldots \ldots \ldots \ldots \ldots \ldots .6 .36$

6.8.6 Localized Failure Parameters . . . . . . . . . . . . . 6 6-36

6.8.7 Assignment of Localized Failure Parameters to Each Container ... . 6 6-38

6.9 Wasteform Parameters $\ldots \ldots \ldots \ldots \ldots \ldots \ldots \ldots \ldots \ldots \ldots \ldots \ldots .6 .41$

6.9.1 Number of Waste Types . . . . . . . . . . . . . . 6-41

6.9.2 Finite Difference Model Release Rate Parameters . . . . . . . . 6-41

6.9.3 Mixing Cell Model Release Rate Parameters ............. 6-46

6.9 .4 Initial Inventory . . . . . . . . . . . . . . . . . 6-47

6.9.5 Waste Type Assignment to Each Container . . . . . . . . . . . 6-48

6.10 External Source/Sink Terms $\ldots \ldots \ldots \ldots \ldots \ldots \ldots \ldots \ldots \ldots .6 .49$

6.10 .1 Number of Source Nodes . . . . . . . . . . . . . . . 6-50

6.10 .2 Number of Source Profiles . . . . . . . . . . . . . . . 6-50

6.10 .3 Number of Source Data Points . . . . . . . . . . . . . . 6-50

6.10 .4 Times and Values of the Sources $\ldots \ldots \ldots \ldots \ldots \ldots \ldots \ldots 6-50$

6.10 .5 Source Locations . . . . . . . . . . . . . . . . 6-51

6.10 .6 Source Type Assignments $\ldots \ldots \ldots \ldots \ldots \ldots \ldots \ldots \ldots \ldots 6.52$

6.11 Create an Input Deck for Use by the DUST Code $\ldots \ldots \ldots \ldots \ldots \ldots 63$

6.12 Store Partial Input in a BLOKXXX File . . . . . . . . . . . . . 6-53

6.13 Exit the Program ........................... $6-54$

7. STRUCTURE OF A DUST INPUT DECK $\ldots \ldots \ldots \ldots \ldots \ldots \ldots \ldots \ldots \ldots$

7.1 DATA SET 1: Title and General Problem Definition (Menu 1) $\ldots \ldots \ldots$ 7-10

7.2 DATA SET 2: Time Parameters (Menu 2) .............. 7-11

7.3 DATA SET 3: Material Assignments/Properties (Menu 3) . . . . . . . 7-12

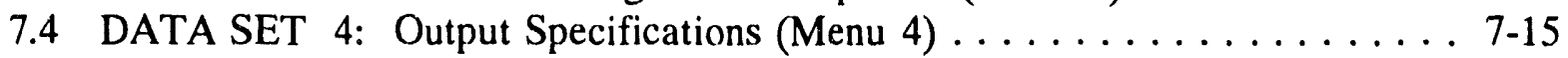

7.5 DATA SET 5: Facility Co-ordinate Data (Menu 5) $\ldots \ldots \ldots \ldots \ldots \ldots$ 7-16

7.6 DATA SET 6: Initial and Boundary Conditions (Menu 6) . . . . . . . 7-17

7.7 DATA SET 7: Water Flow Parameters (Menu 7) . . . . . . . . . . 7 7-19

7.8 DATA SET 8: Container Parameters (Menu 8) ............ 7-20

7.9 DATA SET 9: Wasteform Leaching Parameters (Menu 9) $\ldots \ldots \ldots \ldots .7-23$

7.10 DATA SET 10: External Sources (FD Model only) $\ldots \ldots \ldots \ldots \ldots \ldots$ 7-26 


\section{TABLE OF CONTENTS}

(continued)

PAGE

8. DUST OUTPUT FILES $\ldots \ldots \ldots \ldots \ldots \ldots \ldots \ldots \ldots \ldots \ldots \ldots \ldots$ 8-1

8.1 Primary Output File $\ldots \ldots \ldots \ldots \ldots \ldots \ldots \ldots \ldots \ldots \ldots \ldots$ 8-1

8.2 Concentration Trace File (TRACECND.DAT) $\ldots \ldots \ldots \ldots \ldots \ldots \ldots \ldots .8 .9$

8.3 Flux Trace File (TRACEFXD.DAT) $\ldots \ldots \ldots \ldots \ldots \ldots \ldots \ldots \ldots \ldots 8-11$

8.4 Concentration File (CONCNT.DAT) $\ldots \ldots \ldots \ldots \ldots \ldots \ldots \ldots \ldots \ldots .6 \ldots \ldots$

8.5 Wasteform Release Data (LEACHRL.DAT) . . . . . . . . . . . 8-16

9. INSTRUCTIONS FOR USE OF GRAFXT $\ldots \ldots \ldots \ldots \ldots \ldots \ldots \ldots \ldots .9 .1$

9.1 Selection of the File for Plotting $\ldots \ldots \ldots \ldots \ldots \ldots \ldots \ldots \ldots \ldots .1$

9.2 Selection of the Plot Variables $\ldots \ldots \ldots \ldots \ldots \ldots \ldots \ldots \ldots \ldots \ldots .9 .1$

9.2.1 Number of plots per graph - Independent variable is time $\ldots \ldots \ldots .9-2$

9.2.2 Number of plots per graph - Independent variable is distance ...... 9-3

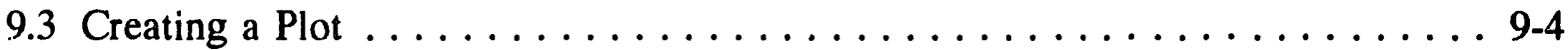

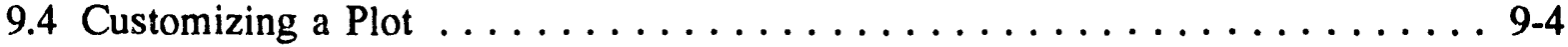

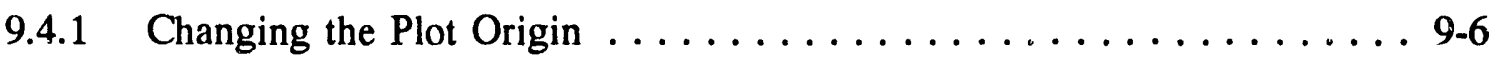

9.4.2 Changing the Axis Labels $\ldots \ldots \ldots \ldots \ldots \ldots \ldots \ldots \ldots \ldots . \ldots \ldots$

9.4.3 Limiting the range of the data $\ldots \ldots \ldots \ldots \ldots \ldots \ldots \ldots .6 .6$

9.5 Creating Additional Plots $\ldots \ldots \ldots \ldots \ldots \ldots \ldots \ldots \ldots \ldots \ldots . . \ldots \ldots$

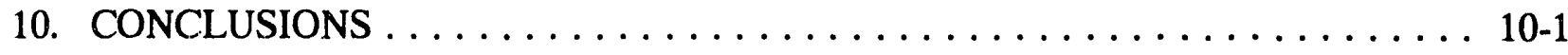

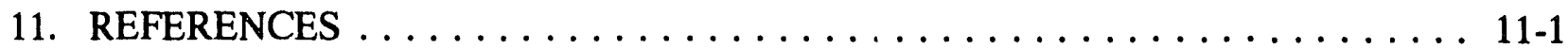

APPENDIX A: Mathematical Description of the Models Selected for Source Term Analysis . . . . . . . . . . . . . . . . A-1

APPENDIX B: Flowchart for the DUST Code $\ldots \ldots \ldots \ldots \ldots \ldots \ldots \ldots \ldots$ B-1

APPENDIX C: Data Requirements for the Pitting Model in Subroutine Breach $\ldots \ldots \ldots$ C-1 


\section{LIST OF FIGURES}

PAGE

Figure 1.1 Schematic diagram of a LLW Disposal Unit $\ldots \ldots \ldots \ldots \ldots \ldots \ldots \ldots$

Figure 2.1 Schematic representation of the mixing cell cascade approach.

(a) a single mixing cell, (b) multiple mixing cells $\ldots \ldots \ldots \ldots \ldots .2-3$

Figure 3.1 Flowchart of the procedure used to take waste stream/wasteform/ container inventory data and define the necessary input parameters to estimate the release rate from a disposal facility $\ldots \ldots \ldots \ldots \ldots .3-2$

Figure 3.2 Schematic outline of the procedure used to analyze release from a LLW disposal facility using the DUST code package . . . . . . 3-4

Figure 5.1 Test case 1, comparison of the DUST finite difference model predictions with the analytical solution for a pulse source at $\mathrm{x}=0$ for 5 days. Retardation and decay are modeled. . . . . . . 5-3

Figure 5.2 Test case 2, comparison of the DUST finite difference model predictions with the analytical solution for a pulse source at $\mathrm{x}=0$ for 5 days. The decay term is zero in this simulation $\ldots \ldots \ldots 5-4$

Figure 5.3 Test case 3, comparison of the DUST finite difference model predictions with the analytical solution for a uniform initial concentration, zero total flux at the boundary $\mathrm{x}=0$, and an external uniform source. ...................... 5-5

Figure 5.4 Test case 4, comparison of the DUST finite difference and mixing cell cascade model at three locations. There are 12 wasteforms, each one meter in length separated by one meter of soil beginning at $x=0$. The container failure time differs for the wasteforms as described in Table 5.2.

Release and transport parameters can also be found in

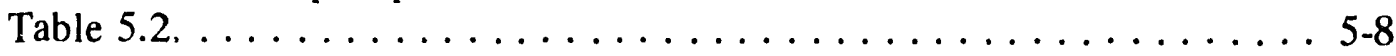

Figure 7.1 Plot of concentration versus time 3 and $23 \mathrm{~cm}$ beneath the last wasteform for the test problems in Table 7.1 (FD)

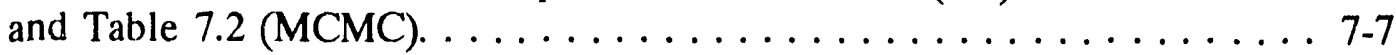

Figure 9.1 Typical graph created by the program GRAFXT $\ldots \ldots \ldots \ldots \ldots .5$ 


\section{LIST OF TABLES}

$\underline{\text { PAGE }}$

Table $2.1 \quad$ Model Selection Summary $\ldots \ldots \ldots \ldots \ldots \ldots \ldots \ldots \ldots \ldots .2-20$

Table 5.1 Parameters used to verify the finite difference transport model $\ldots \ldots \ldots$ 5-2

Table 5.2 Parameters used in test case 4: Comparison of the mixing cell and finite difference model results $\ldots \ldots \ldots \ldots \ldots \ldots \ldots \ldots \ldots, 5-6$

Table 7.1 Sample finite difference input deck for a single material with containers failing at either 5 or 6 years. Release mechanisms include rinse and uniform dissolution. Blank cards are denoted by the word BLANK in columns $1-5 \ldots \ldots \ldots \ldots \ldots \ldots \ldots \ldots \ldots$

Table 7.2 Sample multi-cell mixing cascade input deck for a single material with containers failing at either 5 or 6 years. Release mechanisms include rinse and uniform dissolution. Blank cards are denoted by the word BLANK in columns $1-5 \ldots \ldots .7-5$

Table 7.3 Input cards required to specify an external source $\ldots \ldots \ldots \ldots \ldots .7-28$

Table 8.1 Typical primary output file when the FD transport model is used $\ldots \ldots$ 8-2

Table 8.2 Typical primary output during the computation when the MCMC transport model is used $\ldots \ldots \ldots \ldots \ldots \ldots \ldots \ldots \ldots \ldots . . \ldots$ 8-10

Table 8.3 Typical concentration trace file, TRACECND.DAT $\ldots \ldots \ldots \ldots \ldots$ 8-12

Table 8.4 Typical flux trace file, TRACEFXD.DAT $\ldots \ldots \ldots \ldots \ldots \ldots \ldots$ 8-14

Table 8.5 Typical concentration versus location file at fixed times, CONCNT.DAT . 8-15

Table 8.6 Typical wasteform release output file, LEACHRL.DAT . . . . . . . 8-17 


\section{EXECUTIVE SUMMARY}

The U.S. Nuclear Regulatory Commission (NRC) Regulation 10 CFR Part 61, "Licensing Requirements for Land Disposal of Radioactive Waste," requires that after disposal of low-level wastes (LLW) there is reasonable assurance that the general public will not receive annual off-site doses in excess of 25 millirems to the whole body, 75 millirems to the thyroid, and 25 millirems to any other organ.

Demonstration that these regulatory limits are not exceeded requires the quantitative assessment of the potential radiological impact of a LLW disposal facility on the surrounding environment. Evaluation of these impacts is accomplished through a performance assessment which includes estimates of the following processes for each radionuclide: (a) the rate of release from the disposal unit (i.e., the source term); (b) the transport from the disposal unit to the accessible environment; and (c) the conversion of the radionuclide concentration at the receptor site into an equivalent dose.

The objective of this project is to provide a computer model that estimates the radionuclide release rate from the disposal facility, that is, the source term. General guidelines used while developing the computer model included: use of a modular structure to allow further refinements, limiting the complexity of the models to permit the code to be capable of running quickly on a desktop computer system, and including the flexibility to handle a wide variety of situations typically encountered in LLW disposal.

In general, the source term is influenced by the radionuclide inventory and its origin (i.e., waste stream), the wasteforms and containers used to dispose of the inventory, and the physical processes that lead to release from the facility. The complexity of the problem and the absence of appropriate data prevent development of an entirely mechanistic representation of radionuclide release from a disposal facility. Typically, a number of assumptions, based on knowledge of the disposal system, are used to simplify the problem. The assumptions used while selecting the models to represent radionuclide release from the disposal facility and the rationale for these assumptions was presented in a previous report in this program [Sullivan, 1991a].

The models selected to represent the four major processes (fluid flow, container degradation, wasteform leaching, and radionuclide transport) influencing release have been incorporated into the computer code DUST, Disposal Unit $\underline{\text { Source }} \underline{\mathrm{T}} \mathrm{erm}$, and are described in this report.

The DUST code permits the user to select from two different methods of calculating the transport of radionuclides through the facility, the Multi-Cell Mixing Cascade (MCMC) model and the one-dimensional finite difference (FD) model. The MCMC model is an analytical solution of the advective transport equation with radioactive decay and chemical retardation for constant flow and material properties. The model does permit a unique time to container failure 
and wasteform release rate for each mixing cell having a container. The MCMC model requires relatively little computer time to operate. The FD model solves the transport equation with the processes of advection, dispersion, retardation, and radioactive decay. It is capable of modeling a wider range of conditions than the MCMC model as it permits non-uniform flow and material properties, however, it requires substantially more input and computer time. A complete discussion of the differences between the two models is presented in this report.

The DUST code models fluid flow through tabular input of the flow velocity versus time when using the FD model. For the MCMC model, a time-independent flow velocity must be specified.

Container degradation is modeled in both transport models through a unique container failure time. The value for this parameter should be selected based on the materials and expected environment. In addition, the FD model permits localized container failure. In the localized failure model, a fraction of the container becomes breached prior to total failure. This permits an earlier release of contaminants from the wasteform.

Wasteform release is modeled through three release mechanismis: a surface rinse process in which radionuclides are released upon contact with the solution, partitioning between the wasteform and solution can be modeled when using the FD transport model; diffusion controlled release from the wasteform (FD transport model only); and uniform release in which a fixed fraction of the inventory is released every year. All of these release mechanisms account for radioactive decay of the source. In addition, for the uniform and surface rinse mechanisms a check is performed to insure that releases do not cause concentrations to exceed a user defined solubility limit.

To ease the burden of creating an input deck a pre-processor, DUSTIN, was written. DUSTIN is a menu driven program that guides the user through all of the necessary steps of creating an input deck. Use of DUSTIN permits the user to create an input deck without knowing the formatted structure used by the DUST code. A complete description of DUSTIN and its operation is provided in this report. To allow direct modification of an input deck, a complete description of the formatted structure of a DUST input deck is also provided.

To facilitate the analysis of the output from the code, the program GRAFXT was written. GRAFXT is a menu driven program that reads files created by DUST and plots the data on a video display unit. Graphs of the concentration, flux, and total mass that has passed through a region can be displayed as a function of time or spatial location.

The DUST code has received extensive testing and verification. DUST code predictions have been compared to known analytical solutions as well as other code predictions. A discussion of several of these verification tests is provided. 
The DUST code improves upon existing models in that more flexibility is allowed in modeling the various waste stream/wasteform/container systems while still retaining relatively simple models that do not require extensive computer time or provide an undue burden on the code user in terms of input requirements. However, the models used within the DUST code are based on a number of assumptions. A complete discussion of the limitations that result from the assumptions is presented.

In using DUST, as with all computer models, the validity of the predictions relies heavily on the validity of the input parameters. Often, the largest uncertainties arise from uncertainty in the input parameters. Therefore, it is crucial to document and support the use of these parameters.

The DUST code, because of its ability to compute release rates quickly, will be extremely useful for screening to determine the radionuclides released at the highest rate, parameter sensitivity analyses, and, with proper choice of the input parameters, provide upper bounds to release rates. 


\section{INTRODUCTION}

The U.S. Nuclear Regulatory Commission (NRC) issued the regulation 10 CFR Part 61, "Licensing Requirements for Land Disposal of Radioactive Waste" [FR, 1982]. 10 CFR Part 61 requires, in part, that any "proposed disposal site, disposal facility design, land disposal facility operations (including equipment, facilities and procedures), disposal site closure, and postclosure institutional control are adequate to protect the public health and safety..." Protection of the public is judged by requiring that releases which may occur must be demonstrated with reasonable assurance not to exceed an equivalent dose of "25 millirem to the whole body, 75 millirems to the thyroid, and 25 to any other organ of any member of the public." This requires the quantitative; assessment of the potential impacts of a low-level radioactive waste (LLW) disposal facility on the surrounding environment. In particular, estimation of the dose to the maximally exposed individual is required.

Estimation of the dose to man is accomplished through a performance assessment. A proposed strategy for conducting such an assessment has been presented by the NRC [Starmer, 1988]. Further guidance will be provided by the NRC in their Branch Technical Position on Performance Assessment, preparation of which is in progress. Work :nder this project supports activities in the development of additional guidance. In the proposed performance assessment strategy, performance assessments are conducted through combining a series of separate calculations. These calculations include estimating the rate of radionuclide release from a disposal unit, (i.e., the source term), the transport of the radionuclide from the disposal unit to the accessible environment, and, finally, the conversion of the radionuclide concentration at the receptor site into an equivalent dose. Examples of this approach san be found in the reports produced for the NRC by the staff at Sandia National Laboratory [Kozak, 1989; Kozak, 1990: Chu, 1991].

The objective of this project is to provide computer models that estimate the radionuclide release rate from the disposal facility. A disposal facility is a complex, heterogeneous collection of wastes/wasteforms/containers, soils, and engineered structures (concrete vaults, backfill, vault covers, drains, etc.). For most radionuclides, release from this disposal unit is controlled by access of water to the wasteform, release from the wasteform, and transport to the disposal unit boundary. These processes are influenced by the design of the disposal unit, hydrology, geochemistry and wasteform/container characteristics. A disposal unit is schematically depicied in Fig. 1.1 and typically contains a multi-layered cover to divert water away from the waste; an engineered barrier to further reduce water flow to the wastes (for trench disposal there is no engineered barrier); and metallic, concrete, or HDPE waste containers. The waste comes in many forms including solidified in cement, dewatered resins, activated metals, dry solids (e.g. contaminated paper, cloth, rubber, plastic, glass, etc.). 


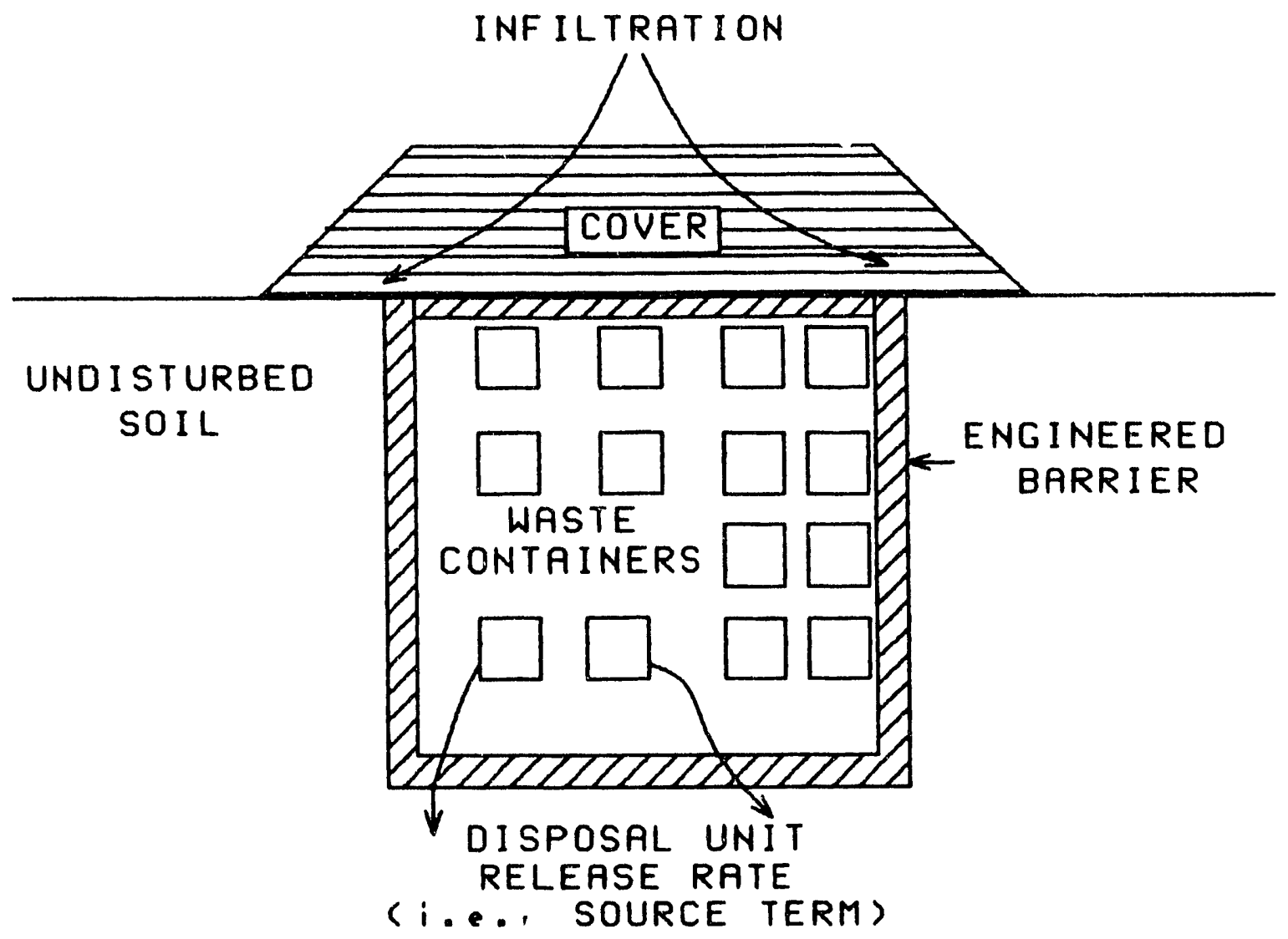

Figure 1.1 Schematic diagram of a LLW Disposal Unit. 
The heterogeneity and complexity of the disposal facility make development of a threedimensional time-dependent model that covers all possible physical and chemical conditions an extremely cumbersome and difficult task. Even if one could develop models for all conditions, their applicability would be restricted by data limitations and the extensive computer time that would be needed.

Therefore, simplifications are justified. The simplified models should account for the most important physical processes and parameters influencing release while retaining as much accuracy as possible. Further, the models should be flexible enough to simulate the wide range of anticipated conditions and not be overly conservative.

Previous reports on this program, [Sullivan, 1991b; Cowgill, 1992; Cowgill, 1992a], presented a review of waste disposal practices, physical and chemical processes that influence release from the facility, reviewed other source term modeling efforts, and recommended models for incorporation into a source term computer model. These models have been embodied into the computer code DUST, Disposal Unit Source Term.

Chapter 2 provides a description of the mathematical models and governing equations used to represent water "iow, container degradation, wasteform leaching, and contaminant transport. A discussion of the range of the parameters in the various models is also provided.

Chapter 3 discusses the procedure used to operate the DUST code. In addition to DUST, a pre-processor, DUSTIN, was written to assist the code user in creating an input deck and a post-processor, GRAFXT, was written to take output files generated by DUST and plot them on the video display unit.

Chapter 4 discusses the limitations of the models.

Several test problems with known analytical solutions have been simulated with the DUST code. The results of these verification tests are presented in Chapter 5.

Chapter 6 presents detailed instructions on how to use the pre-processor DUSTIN. This code is menu driven and asks the user for all of the variables needed to run DUST. DUSTIN will take this information and write it to a file in the form required by DUST, thereby, eliminating the need to know the exact structure of an input file. This chapter also discusses the input parameters and provides guidance on their selection.

Chapter 7 discusses the format of the input required by DUST. This permits the user to create or modify an input deck without using DUSTIN. Sample input decks are provided and discussed. 
Chapter 8 discusses the various output files created by DUST. In addition to the main output file, which contains the problem definition and the requested output (concentrations, fluxes, and wasteform mass release rates), DUST creates files containing concentration, flux, or total mass that has moved past a given location for use with GRAFXT, and files containing information on the wasteform release rates.

Chapter 9 discusses the use of GRAFXT, the graphics program for DUST output files, TRACECND.DAT, TRACEFXD.DAT, and CONCNT.DAT.

Chapter 10 provides a brief summary and conclusion for this report. 


\section{MODEL SELECTION AND GOVERNING EQUATIONS}

A LLW disposal unit is a complex, heterogeneous collection of wastes/ wasteforms/containers, soils, and engineered structures (clay caps, concrete vaults, drains, etc.). Aqueous release of radionuclides from this disposal unit is controlled by water flow, access of the water to the wasteform, release of the radionuclide from the wasteform, and transport to the disposal unit boundary. These processes are influenced by the design of the disposal unit, precipitation, hydrology, geochemistry, and wasteform/container characteristics. To model the complete disposal unit, including every waste container individually would require a three dimensional model that considered all of these processes simultaneously. Such a model does not exist today. Even if such a model did exist, its use would require extensive computing times and the accuracy of the predictions would be questionable due to limitations in the data.

Therefore, simplifications from a fully descriptive three-dimensional model are justified. These "simplified" models are a necessary step in developing predictions of the behavior of a LLW disposal site.

The "simplified" models incorporated into DUST account for the most important physical processes and parameters influencing release. Further, the models are flexible enough to simulate a wide range of conditions. For example, multiple container failure and wasteform release rates can be modeled.

The DUST code has been developed in a general manner which allows simulation of the majority of situations expected to occur. However, to account for the possibility of special cases and allow easy modifications of the models within the code to reflect new and better information, the code structure is modular.

In the following sections, the framework for the DUST code is presented. Within that framework, the models for the four processes (water flow, container degradation, wasteform release, and transport) that influence release are discussed separately. This chapter contains the basic equations that govern the release and transport within the facility. However, the detailed equations describing the analytical solutions used in modeling these processes are presented in Appendix A.

\subsection{DUST Code Framework}

The ultimate objective of the DUST code is to predict the rate of release of radionuclides from a shallow land disposal facility. As discussed earlier, this will be accomplished through use of computer models. These models should be simple enough to allow simulation of a large number of cases and flexible enough to allow simulation of a wide range of situations. To achieve this one can use analytical or numerical solution procedures. Analytical solutions often have the advantage of being easier to compute than numerical solutions. Numerical solutions offer the flexibility to model a wider range of conditions. For these reasons both are incorporated into the DUST code. 
The DUST code was written in an attempt to achieve a balance between the use of extremely simple but conservative assumptions which lead to high predicted release rates and complicated models that include all of the known physical and chemical processes that influence release but require r:xtensive computer time and expertise to define the problem (select the input variables). To strike this balance, assumptions regarding which are the most important physical parameters and the level of detail needed to calculate these parameters have been made. These are discussed in detail in the model selection report [Sullivan, 1991]. Also, a pre-processor, DUSTIN, has been written to ease the burden of creating an input deck. DUSTIN is discussed in detail in Chapter 6.

The primary equation for predicting release from the disposal facility is the contaminant transport equation. In addition, there are models that supply information on fluid flow, container degradation and wasteform release. For calculating transport, two models are permitted, the Multi-Cell Mixing Cascade (MCMC) model and the Finite Difference (FD) model. A flow chart of the various subroutines that comprise the DUST code is presented in Appendix B.

The MCMC model divides the modeled domain into mixing cells. Each mixing cell has the same size and transport properties. However, container performance and wasteform release may vary between mixing cells. Using this approach, an analytical solution of concentration versus time may be obtained for any mixing cell [Sullivan, 1991]. This solution is presented in Appendix $\mathrm{A}$.

The FD model divides the modeled domain into finite regions called control volumes. The FD model is a generalization of the MCMC model and permits more flexibility in modeling different situations. In particular, the FD model permits different transport properties in each node, diffusive/dispersive transport, and more general wasteform release models.

The differences between the two transport models leads to substantial differences in selection of the container degradation and wasteform release models. Therefore, the transport models are discussed first.

\subsection{Radionuclide Transport}

Two alternative methods have been selected to model transport within the disposal facility. The multi-cell mixing cascade (MCMC) models [Fig. 2.1] used in the PAGAN performance assessment code [Kozak, 1990; Chu, 1991] have been generalized to allow mnre realistic estimation of the releases from the disposal facility while still retaining an analytical solution procedure. In cases where the assumptions used in obtaining the analytical solutions are not appropriate, a one-dimensional finite difference (FD) model is provided. 

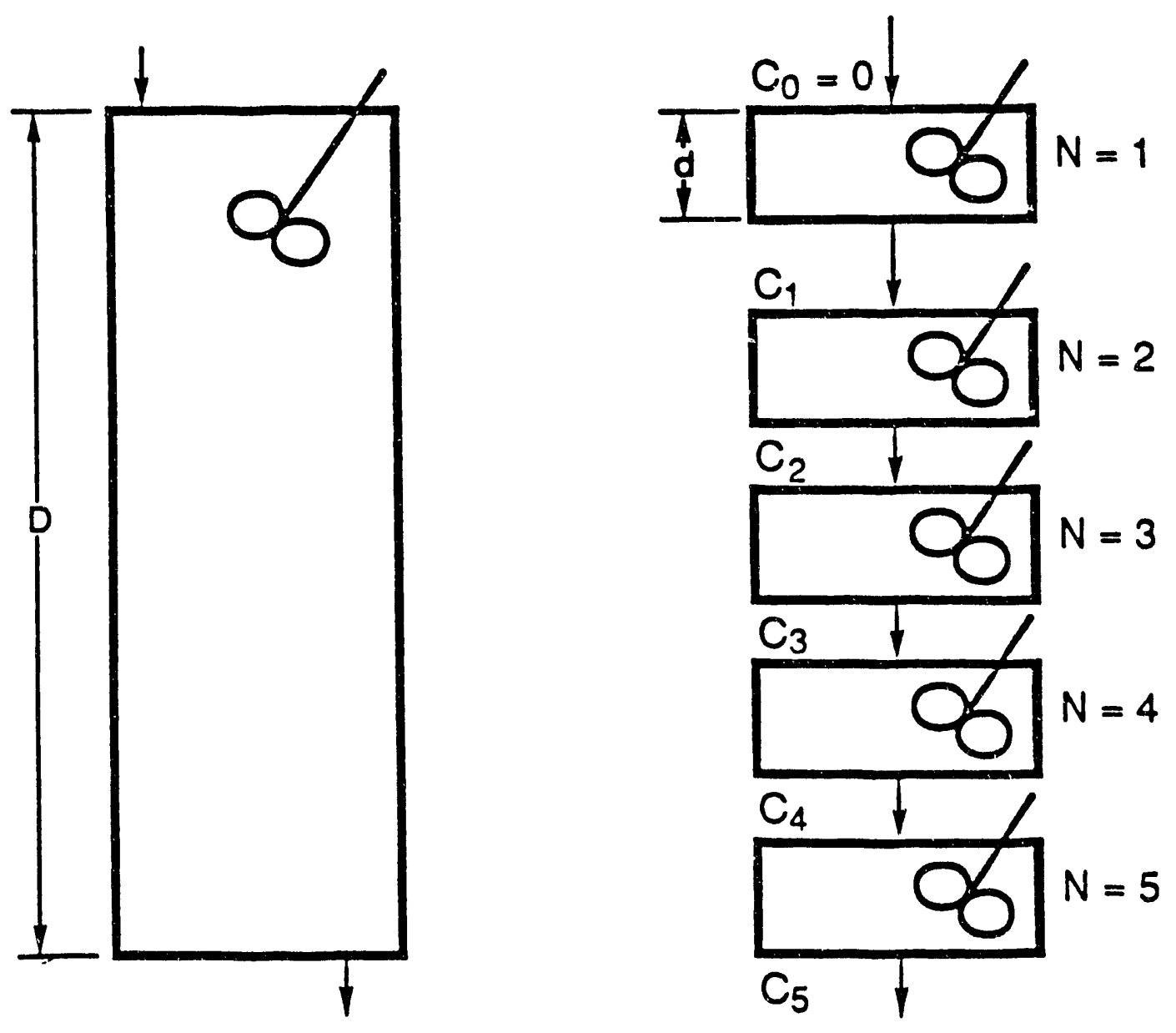

Figure 2.1 Schematic representation of the mixing cell cascade approach. (a) a single mixing cell, (b) multiple mixing cells. [From Kozak, 1990] 
Both models begin with the one-dimensional advection-dispersion transport equation:

$$
\begin{aligned}
\frac{\partial}{\partial t}(\theta C)= & \frac{\partial}{\partial x} \theta D \frac{\partial C}{\partial x}-\frac{\partial}{\partial x} V_{D} C \\
& -\lambda(\theta C+\rho S)-\frac{\partial}{\partial t}(\rho S)
\end{aligned}
$$

where:

$\mathrm{C} \quad=$ solution concentration;

$\theta \quad=$ the volumetric moisture content of the region (dimensionless);

$\mathrm{D} \quad=$ the diffusion-dispersion coefficient,

$$
D=D_{\text {eff }}+\frac{a_{t}\left|V_{D}\right|}{\theta}
$$

$\mathrm{D}_{\text {eff }} \quad=$ effective diffusion coefficient;

$\mathrm{a}_{\mathrm{t}} \quad=$ dispersivity coefficient;

$\mathrm{V}_{\mathrm{i}} \quad$ = Darcy velocity;

$\lambda \quad=$ radioactive decay constant;

$\mathrm{S} \quad=$ adsorbed concentration, the mass adsorbed per unit mass of the solid;

$\rho \quad=$ bulk density of the solid; and

$\mathrm{q} \quad=$ source/sink term used to model release from the wasteform and external sources, e.g., production due to ridioactive decay.

In Eqn. (2.1) we assume that the mass adsorbed on the solid surfaces is in equilibrium with the mass in solution. Further, we assume that this equilibrium can be described using a concentration independent distribution coefficient, $\mathrm{K}_{\mathrm{d}}$, as follows:

$$
S=K_{d} C
$$

Using the above relationship for $\mathrm{S}$, assuming that the bulk density remains constant within the disposal facility and rearranging Eqn. (2.1), the following equation is obtained: 


$$
\frac{\partial}{\partial t}(R \theta C)=\frac{\partial}{\partial x}\left(\theta D \frac{\partial C}{\partial x}\right)-\frac{\partial}{\partial x}\left(V_{D} C\right)-\lambda \theta R C+q
$$

where:

$$
R=1+\frac{\rho K_{d}}{\theta}
$$

$R$ is known as the retardation coefficient. Values of the distribution coefficient $\left(K_{d}\right)$ are known to change by urders of magnitude under different chemical conditions. Therefore, the value selected for $\mathrm{K}_{d}$ must be well supported. Typical $\mathrm{K}_{d}$ values for a range of conditions have been compiled in several reports [Thibault, 1990; Looney, 1987; Baes, 1983].

\subsubsection{FD Model}

The FD model takes Eqn. (2.3) and transforms it into a set of coupled algebraic equations using a control volume approach. In this method, the modeled domain is divided into control volumes. A mass balance is performed for each control volume through approximation of the derivative terms in Eqn. (2.3) using finite differences. The time derivative is estimated using a backward difference procedure. The diffusion/dispersion term is estimated using central differences. The advection term is modeled using an upwind difference. A complete description of the control volume approach and the resulting system of equations is presented in Appendix A.

The FD model is quite general in that in principle each control volume could have a different retardation coefficient, diffusion/dispersion term, moisture content, and source/sink term. (In practice, DUST permits only 10 different material types per calculation.) This flexibility permits simulating the effects of caps, engineered barriers, and backfill using different parameters. In addition, the source/sink term contains two components, a wasteform term and an external term. The wasteform term is calculated using the release models described in Section 2.5.

In the MCMC model, migration is permitted only through advection altered through the effects of retardation in the downward direction. As stated previously, the mixing cell cascade model does not model diffusional or dispersive transport and does not allow $f$ or migration up towards the surface. Diffusional transport may be an important process during the time period when the engineered barriers provide protection against water flowing through the waste containing region of the trench. Dispersion may be important in causing early releases and in spreading the contaminant causing lower peak concentrations. 


\subsubsection{Mixing-Cell Cascade}

The mixing cell cascade model divides the disposal facility into a number of uniform size mixing cells as depicted in Figure 2.1. Within each cell it is assumed that the contaminant released from the waste form is uniformly mixed thereby giving a uniform solution concentration. In order to obtain an analytical solution the following assumptions are made:

a) Migration is dominated by advective flow and therefore diffusion and dispersion can be ignored.

b) The advection velocity, moisture content, and the retardation coefficient are constant throughout the disposal facility. Although these parameters will show variations due to different materials in the facility, they should be selected to provide a representative average for the entire facility. Obtaining a timeindependent average value for the retardation coefficient in a multi-layered system is not a trivial task. Examining Eqn. (2.5) it is seen that during a transient, the importance of the retardation coefficient depends on the gradient in concentration which changes in time. As an example, consider a problem that has a source at the boundary and two different material regions. At times prior to the contaminant arriving in region 2 , the effective system retardation coefficient is that of material 1. As contaminant enters region 2 , its transport characteristics play a role in determining migration and the effective retardation coefficient becomes a function of the region 1 and 2 values. At later times, if region 1 obtains a quasi-steady state $(\partial \mathrm{c} / \partial \mathrm{t}-0)$, the retardation coefficient in this region becomes unimportant and the system retardation coefficient would be close to that in region 2 . In general, if the retardation coefficient within the disposal facility varies greatly from region to region, it is recommended to use the FD transport model. becomes:

Using these assumptions in Eqn. (2.3) the transport equation for the $\mathrm{i}^{\text {th }}$ mixing cell

$$
\frac{\partial C_{i}}{\partial t}=-\frac{V_{D}}{\theta R} \frac{\partial C_{i}}{\partial x}-\lambda C_{i}+\frac{q_{i}}{\theta R}
$$

where the subscript $i$ refers to the $i^{\text {th }}$ mixing cell.

For a disposal facility of height $H$, there are $N$ mixing cells of height $h(h=H / N)$. Using this definition of the size of the mixing cell and upwind differencing (because migration is assumed to be controlled by advection) to estimate the spatial derivative, Eqn. (2.5) becomes: 


$$
\frac{d C_{i}}{d t}=-\alpha N\left(C_{i}-C_{i-1}\right)-\lambda C_{i}+\beta N Q_{i}
$$

where:

$$
\begin{aligned}
& \mathrm{Q}=\text { the total release rate from the wasteform; } \\
& \alpha=\mathrm{V}_{\mathrm{d}} /(\theta \mathrm{RH}) ; \\
& \beta=1 /\left(\theta \mathrm{RHA} \mathrm{A}_{\mathrm{f}}\right) ; \text { and } \\
& \mathrm{A}_{\mathrm{f}}=\text { the area of the facility. }
\end{aligned}
$$

In this description, $\mathrm{HA}_{\mathrm{f}} / \mathrm{N}$ is the volume of a single mixing cell.

Equation (2.6) applies to each mixing cell. For the first cell, $\mathrm{C}_{\mathrm{i}-1}$ is set to zero. This is equivalent to assuming that no contaminant enters through the top of the facility. Therefore, we have a system of $\mathbf{N}$ coupled linear differential equations. This system of equations has been solved to provide an analytical solution for arbitrary wasteform sources, $Q_{i}$, within each mixing cell. The results are presented in Appendix A.

A control volume in the FD approach is equivalent to a mixing cell in the MCMC approach. Often throughout the remainder of this document, the term node is used. This is a generic term for the region of computational interest, i.e., a node can refer to either a mixing cell or control volume. Where possible, the term control volume will be used for cases that apply only to the FD model, mixing cell will be used only for cases involving the MCMC model, and node will be used in cases that apply to both models.

\subsection{Fluid Flow}

Although setting a disposal facility beneath the water table is permitted, it is expected that new facilities will be located above the water table in the unsaturated zone. In either case, the most likely pathway for release will be through the water. Infiltration of water into a facility will involve many processes including precipitation, evapotranspiration, and surface run-off. Water flow in the unsaturated zone is difficult to predict due to the non-linearity of the unsaturated soil flow properties. This is further complicated by the barriers (trench cap, concrete structure, etc) any disposal facility will have to minimize infiltration into the waste containing region.

A simple model for infiltration is needed. In addition, predicting infiltration into soils in arid sites under all conditions is difficult [Gee, 1988]. To calculate flow into a disposal facility would require at least a two-dimensional simulation. Further, the flow rate will vary with time on a short time scale (hours) due to precipitation events and evapotranspiration and on a long time scale (years) due to changes caused by degradation of the infiltration barrier. To 
follow the evolution of water flow with time would require an extensive computing expense. Thus, this is not appropriate for the source term model.

In the DUST code, for the FD transport model, water infiltration is calculated as a function of time through tabular input.

$$
V_{d}=F(t)
$$

where $V_{d}$ is the volumetric flow velocity (Darcy velocity) of the water and $F(t)$ is defined in an input table. If the MCMC transport model is used, the Darcy velocity must be a constant.

This flow rate should be the yearly average based on the expected conditions. For advection driven transport it has been shown that the average rate of contaminant transport depends on the average flow rate [Sullivan, 1988a].

The choice of the value for the flow rate should be conservatively chosen or supported by more detailed computer simulations such as VAM2D [Huyakorn, 1989], FEMWATER [Yeh, 1987], TRACR3D ['Travis, 1991], etc. If a computer simulation is not performed, an upper bound for the flow rate is the annual precipitation rate. If the evapotranspiration rate is accurately known this could be subtracted from the precipitation rate at humid sites. At arid sites this may lead to large errors in predicted recharge [Gee, 1988]. Alternatively, if the recharge rate through the disposal facility is known due to measurement at the site, this value could be used.

In the actual situation, infiltration may be very low until significant degradation of the cap occurs. If one accounts for degradation of the cap, this will require additional modeling. At this time, there is no widely accepted model for the degradation of earthen materials or engineered (i.e., concrete) caps. This is due in part to the need to predict performance over hundreds of years based on experience and data that have been collected over a period of years.

However, work is being performed to determine the degradation mechanisms of underground concrete structures. Models based on these studies are under development [Clifton, 1989; Walton, 1990; Shuman, 1991] and should help in estimating the rate of degradation. This information could then be used to calculate water flow through the degraded barrier and into the waste containing region of the disposal facility.

\subsubsection{Gas Flow}

If it is determined that the gaseous pathway may be significant, the flow of gas through the facility will need to be modeled. Conceptually, the DUST code would handle the problem in an identical fashion to that for water flow, i.e., a table of volumetric gas flow rate versus time could be used for the FD model and a constant value for volumetric gas flow rate could be used 
by the MCMC model. Models for the production and/or release of gaseous phase radionuclides would have to be developed.

The transport of gaseous radionuclides within the disposal facility requires special attention. For gases, flow may be up and out of the disposal facility or out of the bottom through drains. Upward migration will involve advection due to pressure variations that will vary seasonally and diurnally, as well as diffusion. As revealed in the review of existing source term models [Sullivan, 1991a], very little work has been performed in modeling gaseous release for performance assessment calculations.

As with water flow, prediction of the upward migration of gas is a complicated problem. Due to the length of time to be considered in a performance assessment, it is impractical to model daily or seasonal variations in gas flow rate. Therefore, it will be necessary for the code user to supply an average gas advection velocity in order to calculate gaseous release. This flow rate should be estimated using state-of-the-art computer codes or, as a minimum, chosen to permit conservative predictions of gas release.

\subsection{Container Degradation}

Waste containers in the early days of LLW disposal ranged from cardboard and wooden boxes to carbon steel drums and boxes. Since the passage of 10 CFR Part 61, cardboard and wooden boxes are no longer used. As of 1988, carbon steel drums and boxes were widely used to dispose of Class A wastes, the largest volume of wastes. Most Class B and C wastes are disposed of in high-integrity containers (HIC) but a small fraction have been stabilized in cement and placed in 55 gallon drums [Sullivan, 1989]. Recently, there has been a trend to rely more and more exclusively on HIC's for Class B and C wastes due to their ease of use, lack of need for processing equipment, reduced worker exposure, problems encountered with solidification of some waste streams, and their approval by $\mathrm{NRC}$ as a means of demonstrating structural stability.

A waste generator has a number of different HIC's from which to choose. These include HIC's made from Ferralium 255, from stainless steels, from polymer-impregnated concrete, and high-density polyethylene (HDPE). A HIC may also have an internal lining to isolate the waste from the external barrier to water flow. The liner materials are typically polyethylene. HIC's should be designed to maintain their structural stability and maintain a positive seal for 300 years as indicated in the NRC Technical Position on Wasteform [Higginbotham, 1983; Lohaus, 1991]. Structural stability does not imply that the HIC's will remain water tight. In time, water may enter through the passive gas vents required on HIC's or through small cracks and localized failures that may occur.

Currently, most waste containers are metallic. The use of HDPE alone is suspect due to the potential of long term creep affecting its stability and it is no longer on the NRC-approved list of HIC's. HDPE is used as a liner in metallic containers and within a concrete caisson which is backfilled with soil. The Richland site received five of these concrete caisson/HDPE HIC's in 1988 [Sullivan, 1989]. 
Modeling of metallic corrosion on a mechanistic scale is strongly dependent on the local chemistry and quite complicated. For the source term model, the work required to perform such a calculation is not justified. Rather, in the DUST code metallic container degradation models will be semi-empirical and rely on the existing corrosion in soil data base. If internal corrosion is expected to be important, this can also be included in the empirical model, however, data in this area is lacking. In the DUST code, two types of failure are modeled: general failure, and localized failure.

\subsubsection{General Failure}

In the DUST code general failure is modeled through a user-specified time of failure. In this model, the container prevents water ingress to the waste until failure, at which time the container no longer provides a barrier to water flow. For metallic containers, the time to failure could be estimated as the thickness of the container divided by the time-averaged corrosion rate.

Corrosion rates should be obtained from site specific data whenever possible. When this is not possible, the data base generated by the National Bureau of Standards, NBS (currently, National Institute for Standards and Technology) [Romanoff, 1957; Gerhold, 1981] for carbon steels and stainless steels could be used for these materials. There is no data base for the corrosion of Ferralium in soil systems. However, Ferralium, a duplex stainless steel, has shown superior corrosion performance as compared to 304 and 316 stainless steels in a wide range of environments. If this trend holds for soil systems, use of the NBS data for stainless steels should be conservative.

The NBS studies of carbon steels covered a period of 17 years and 47 different soils [Romanoff, 1957]. Uniform corrosion rates in this study of carbon steels ranged from $8 \times 10^{-4}$ $2 \times 10^{-2} \mathrm{~cm} / \mathrm{yr}$, with the mean value being $5.7 \times 10^{-3} \mathrm{~cm} / \mathrm{yr}$. In the LLW Updated Impacts Analysis [Otzunali, 1986] the recommended value for carbon steel corrosion was $4 \mathrm{mils} / \mathrm{yr}\left(1 \times 10^{-2} \mathrm{~cm} / \mathrm{yr}\right)$. For typical 55 gallon carbon steel drum thicknesses, $50 \mathrm{mil}$, container lifetimes would be expected to range from 6 to 160 years with a mean lifetime of 23 years based on the above data.

The NBS studies of 304 and 316 stainless steels were conducted over 14 years in 15 soils. General corrosion rates for 304 stainless steel ranged from $1.7 \times 10^{-5}-1.1 \times 10^{-7} \mathrm{~cm} / \mathrm{yr}$, with the mean value being $5 \times 10^{-6} \mathrm{~cm} / \mathrm{yr}$ [Gerhold, 1981]. Corrosion rates for 316 stainless steel ranged from $5.7 \times 10^{-6}-2.8 \times 10^{-8} \mathrm{~cm} / \mathrm{yr}$, with the mean value being $1.3 \times 10^{-7} \mathrm{~cm} / \mathrm{yr}$ [Gerhold, 1981]. Otzunali recommended a value of $0.3 \mathrm{mils} / \mathrm{yr}\left(7.6 \times 10^{-4} \mathrm{~cm} / \mathrm{yr}\right)$ [Otzunali, 1986]. For a $3 / 8$ inch thick stainless steel container, the lifetime based on the corrosion rate recommended by Otzunali would be 1250 years.

In the soil corrosion experiments, it was noted that corrosion rates typically decreased over time [Romanoff, 1957, Gerhold, 1981]. Provided that there is no change in the degradation mechanism, the experimentally measured decreasing corrosion rate indicates a constant rate based on short term data is likely to overpredict the total amount of corrosion. 
For cortainers with non-metallic components (HDPE containers, polyethylene lining in metrl, concrete caissons, etc.) there aie few data on their long term performance. It is recommended that the general failure rate be selected in a conservative manner based on expert judgement.

Both transport models permit a unique time to failure for every container.

\subsubsection{Localized Failure}

The FD transport model also permits containers to partially fail prior to general failure. The analytical solution for the MCMC model does not accommodate partial container failures.

If localized failure occuis, water will contact the wasteform causing the release of radioactivity bufore the genera! corrosion allowance is reached. This solute may be released immediately after local failure or it may be stored within the container causing a large pulse type release when general failure occurs. In either event it may have a significant impact on predicted releases from the disposal facility.

For metallic HIC's localized failure can occur due to pitting, stress corrosion cracking, failure of the passive gas vents, or other mechanisms. In these failure scenarios, only a small portion of the container will permit water access to the wasteform. The reduced water flow impacts on the amount released and available for transport. This is accounted for in the leaching model.

Iocalized failure is modcled similariy to the approach used the BLT code [Sullivan, 1989]. The model was originally developed for pitting of carbon steel drums. The breached area is estimated from the following equation:

$$
A_{b}=N_{p} A_{c} \pi\left(h^{2}-T^{2}\right)
$$

where:

$N_{F}$ is the number of localized failures pei unit area of the container;

$A_{c}$ is the total container area;

$T$ is the thickness of the metal; and

$h$ is the penetration depth.

If the penetration depth, $h$, is less than the metal thickness, the container has not been penetrated and the breached area is set to zero.

The penetration depth is estimated from the expression:

$$
h=k t^{n}
$$


where $\mathrm{t}$ is time in years, and $\mathrm{k}$ and $\mathrm{n}$ have been determined for carbon steel [Mughabghab, 1988] based on the NBS corrosion data in soils [Romanoff, 1957; Gerhold, 1981]. For carbon steel, the parameter $\mathbf{k ~ w}$.Js found to depend on the soil-water $\mathrm{pH}$ and the parameter $\mathrm{n}$ depends on the degree of soil aeration, moisture content, and clay content. The value for $n$ is always less than 1 and is higher for poorly aerated soils (poor drainage) as compared to well aerated soiis.

A detailed discussion of the choice of values for $N_{p}, k$ and $n$ can be found in the BLT data input guides [Sullivan, 1989], which is reproduced in Appendix C. For carbon steels, $\mathrm{N}_{\mathrm{p}}$ was found to range from 0.05 to 0.5 per $\mathrm{cm}^{2}$, the average value for $\mathrm{k}$ was $0.0457 \mathrm{~cm} / \mathrm{yr}$ and $\mathrm{n}$ ranged from 0.01 to 0.93 . Typical values for these parameters are discussed in Section 6.8.6.

For stainless steels, even though there are 14 years of corrosion data in 15 different soils [Romanoff, 1957; Gerhold, 1981], the data are insufficient to support estimation of the necessary parameters. Thus, for these and other container materials, the parameters will have to be estimated using engineering judgement if localized corrosion is modeled.

Through proper choice of the localized corrosion parameters, failure of the passive gas vents required on HIC's may also be modeled. For example, by setting $\mathrm{n}$ to zero and appropriate choice of the parameters $h$ and $N_{p}$, a constant area of failure may be estimated. This failure area may be particularly important if gaseous release is being modeled.

Consideration should also be given to internal corrosion. HIC's may store wastes without the waste undergoing a solidification process. In this case, the wastes may directly contact the lining or, in the absence of a lining, the container material. Many LLW wastes contain corrosive agents that could possibly lead to penetration via pitting (localized failure). For example, it has been shown that resin beads in contact with stainless steel led to discoloration and pitting in short term tests [Soo, 1990].

\subsection{Wasteform Leaching}

Radionuclide release from the wasteform commences upon container failure. In a LLW facility there will be several different wasteforms, a partial list of which includes: wastes solidified by one of several processes (cement, VES, bitumen); activated metals; compacted lab trash; dewatered resins; liquids contained in an absorbent; and adsorbed gases [Roles, 1990]. The disposal data must be analyzed in order to determine the most likely release mechanisms for each radionuclide. After analyzing the data, each of the major categories of wasteforms should be grouped in terms of release mechanism. Review of the disposal data shows the following major waste streams:
a) activated metals;
b) dry active waster;
c) resins; and
d) filter media 
Approximately $70 \%$ of the activity is in activated metals, $15 \%$ of the activity is solidified in cement, and most of the rest is dry active wastes [Sullivan, 1991b]. However, the distribution by radionuclide changes markedly from one radionuclide to the next. For example, over $80 \%$ of the Th-232 is disposed of with sorbents, over $50 \%$ of the $\mathrm{C}-14$ is in cement, etc [Cowgill, 1992]. Therefore, the user must determine the distribution on a radionuclide specific basis.

Based on the above groups the following release mechanisms will be modeled:
a) Solubility limited;
b) Surface wash-off subject to partitioning;
c) Diffusion; and
d) Uniform (e.g., Dissolution).

In general, a wasteform may release radionuclides by more than one mechanism. This will be allowed through user-supplied input. In particular, the user will be allowed to specify the fractional amount of mass released by each mechanism. For examplt the user could specify that for $10 \%$ of the mass, release is controlled by partitioning, while the other $90 \%$ is controlled by diffusion. This flexibility may prove to be important when homogenizing the number of waste streams/wasteforms or in modeling large boxes containing many wasteforms.

\subsubsection{Solubility-limited Release}

Solubility-limited release will be modeled by allowing an instantaneous release of radionuclides into solution until the limit is reached or the entire inventory is released. Further, if a solubility limit is specified and other release mechanisms are used to predict release, the amount released will be constrained such that the solubility limit is not exceeded. In general, the chemistry that occurs within a disposal facility is complex and changes in time due to the degradation of the waste containers and wasteforms. Obtaining reliable solubility limits in this environment is a difficult task. Any choice of solubility limits must be justified as conservative under all of the potential conditions. For this reason, the default solubility limit in the DUST code is arbitrarily set to $10 \mathrm{gm} / \mathrm{cm}^{3}$. This value is high enough to insure that solubility limits do not influence release. Representative ranges of solubility limits have been compiled and are presented in [Looney, 1987].

\subsubsection{Surface Rinse with Partitioning}

The surface rinse model assumes that the radionuclides in the wasteforms with this release characteristic are available for release as soon as water contact occurs. Prior to container failure the radionuclides may be held on the wastes by adsorption, chemisorption, adhesion, and ionexchange among other factors. To account for these factors a partition factor, which is an equilibrium ratio relating the amount on the wasteform to that in solution, can be used. This partition factor depends on the properties of the wasteform and the local chemistry. The partition factor is a lumped parameter that covers many physical processes. Therefore, obtaining reliable estimates may be difficult. 


\subsubsection{MCMC Model}

Use of the MCMC transport model requires a single retardation coefficient representative of the entire disposal facility. Therefore, the partition coefficient in the rinse model is identical to the system distribution coetficient. It is a global parameter that reflects a system average value, accounting for the different materials within the disposal facility (soil, wasteforms, containers, engineered barriers, etc.).

Equilibrium between the solution and the solids is assumed to occur instantly after container breach at time $\tau_{b}$. One method for expressing this release rate, $Q$, in Eqn. (2.6) is:

$$
Q=M(t) \delta\left(t-\tau_{b}\right)
$$

where:

$$
\begin{array}{ll}
\mathbf{M}(t) & =\mathbf{M}_{r} \mathrm{e}^{-(\lambda t)} / \mathbf{R} ; \\
\mathbf{M}_{r} & =\text { rinse mass available at } t=0 ; \\
\lambda & =\text { radioactive decay constant; } \\
\mathbf{R} & =\text { retardation coefficient }=1+\rho \mathrm{K}_{\mathrm{d}} / \theta ; \\
\mathbf{K}_{\mathrm{d}} & =\text { distribution coefficient; } \\
\boldsymbol{\theta} & =\text { moisture content; and } \\
\rho & =\text { bulk density of the solids. }
\end{array}
$$

The model assumes that all of the rinse mass is released upon breach. Equation (2.10) calculates the amount that enters solution (the rest of the mass is adsorbed on the solids). As mass in solution is removed due to transport or decay, mass adsorbed to the solids is released to solution to maintain the local equilibrium. In the present form, Eqn. (2.10) is useful in the mixing cell cascade model which analytically integrates the release term. However, due to the $\delta$ function, it is not useful for the finite difference model.

\subsubsection{FD Model}

For the finite difference model, the mass released to solution is calculated by requiring equilibrium between the solid and solution to be maintained at the beginning of each time step. As mass is transported away over the numerical integration time step, equilibrium is no longer maintained. Therefore, the procedure is repeated at the beginning of each time step. This following expression for the mass release rate to solution arises: 


$$
q(t)=\frac{M(t)\left\lceil 1-\frac{C}{C_{s a t}}\right]}{\theta V \Delta t}
$$

where:

$$
\begin{array}{ll}
\mathrm{q}(\mathrm{t}) & =\text { release rate per unit volume; } \\
\Delta \mathrm{M}(\mathrm{t}) & =\text { mass released at time } \mathrm{t} \text { to maintain equilibrium; } \\
\mathrm{C}(\mathrm{t}) & =\text { solution concentration at time } \mathrm{t} ; \\
\mathrm{V} & =\text { volume of the finite difference node; and } \\
\Delta \mathrm{t} & =\text { time step size. }
\end{array}
$$

After performing the mass balance at the beginning of the time step, $M(t)$ can be estimated from [Sullivan, 1991]:

$$
\Delta M(t)=\frac{\left[M_{r}(t)-\frac{\rho K_{p}}{\theta} M_{s}(t)\right]}{\left[1+\frac{\rho \cdot K_{p}}{\theta}\right]}
$$

where:

$\mathrm{K}_{\mathrm{p}} \quad=$ the wasteform partitioning coefficient. In the FD Model the soil distribution coefficient may differ from the wasteform partition coefficient. Also, each wasteform may have a unique partition coefficient.

$M_{r}(t)=$ the rinse mass available at time $t$, which is the original rinse mass minus any mass that has been released or lost to radioactive decay.

Also,

$$
\mathrm{M}_{\mathrm{s}}(\mathrm{t})=\mathrm{C}(\mathrm{t}) \theta \mathrm{V}=\text { mass in solution at time } \mathrm{t} .
$$




\subsubsection{Diffusion Release Model (FD Model Only)}

Experimental leaching data from solidified wastes often indicate that diffusion is the rate controlling process. In fact, the ANS 16.1 standard leach test interprets the data in terms of diffusion [ANS, 1986].

Diffusion-controlled release is characterized by relatively high leach rates at early times which continually decrease over time. In fact, analytically, the release rate, although it is integrable, approaches infinity as time approaches zero. For this reason, a release model based on a constant release rate may prove to be difficult to justify for diffusion controlled release. Choosing a constant release rate based on short term releases may be overly conservative while choosing the rate based on some type of average value may underpredict early releases.

The diffusion model will consider the two geometries used most widely in LLW disposal: cylindrical (drums) and rectangular (boxes). To simplify the situation, it will be assumed that the concentration in the contacting solution is zero. That is, solution feedback effects are ignored. This assumption leads to the highest predicted release rates and permits an analytical solution to ke obtained.

In both models, we analytically solve the diffusion equation corrected for decay.

$$
\frac{\partial C}{\partial t}=\nabla \cdot D \nabla C-\lambda C
$$

where $D=$ the effective diffusion coefficient and all other parameters have been previously defined.

The initial condition assumes a uniform concentration throughout the wasteform:

$$
C(x, y, z, 0)=C_{\text {o }}
$$

The boundary conditions assume symmetry about the midplane of the wasteform and zero concentration at the outer edge.

$$
\begin{aligned}
& C\left(x_{b}, y, z, t\right)=0 \\
& C\left(x, y_{b}, z, t\right)=0 \\
& C\left(x, y, z_{b}, t\right)=0
\end{aligned}
$$


where the subscript b denotes a boundary.

Solution of Eqn. (2.13) subject to the initial and boundary conditions gives the concentration at any location within the wasteform. However, the quantity of interest is the release rate, which is the mass flux integrated over the surface area.

$$
Q(t)=\int d S \cdot J_{s}
$$

where:

$Q(t)$ is the mass release per unit time; and

$\mathrm{J}_{\mathrm{s}}$ is the mass flux at the surface.

For one-dimensional diffusion-controlled release,

$$
J_{s}=-D \frac{\partial C\left(x_{s}\right)}{\partial x}
$$

where $x_{s}$ denotes a surface of the wasteform.

Equation (2.13) is solved analytically and used to evaluate the flux as prescribed by Eqn. (2.17). This expression is placed in Eqn. (2.16) and the release rate is determined. The detailed expressions for release rate for both geometries can be found in Appendix A.

\subsubsection{Uniform Release Model}

The uniform release model assumes that the wasteform release rate decreases in time due only to radioactive decay and solubility constraints. In the Source Term Model Selection report [Sullivan, 1991a] (and in the computer code BLT), this model is named the dissolution model and was meant to represent releases from activated metals which undergo corrosion. Due to the wide variety of waste streams/wasteforms which this model may be used to represent and to avoid confusion with the term dissolution which has a specific definition, the name has been generalized to uniform release model. 
In the uniform release model, the release rate is:

$$
Q=Q_{f} M_{w f} e^{-\lambda t}\left[1-\frac{C}{C_{s e x}}\right]
$$

where:

$\mathrm{Q}_{\mathrm{f}}=$ fractional release rate, and

$\mathrm{C}=$ solution concentration at time $\mathrm{t}$.

$\mathbf{M}_{\mathrm{wf}}=$ initial mass of the contaminant in the wasteform.

and all other variables have been previously defined. Equation (2.18) is analytically integrated over a time step and the resulting expression provides the total mass release in that time step.

The fractional release rate is the fraction of the initial mass in the wasteform that is released per unit time. In the BLT code, the dissolution release model is:

$$
Q=\left(\frac{u M_{w f} S}{V_{w f}}\right)\left(1-\frac{C_{s}}{C_{s a t}}\right) e^{-\lambda t}
$$

where $u$ is the dissolution velocity, $S$ is the surface area, and $V_{w f}$ is the volume of the wasteform. Comparing the two expressions, it can be seen that for dissolution release, the fractional release rate is:

$$
Q_{f}=\frac{u S}{V_{w f}}
$$

If dissolution release is the appropriate model, Eqn (2.20) can be used to estimate the fractional release rate.

\subsubsection{Influence of Localized Failure on Release}

If there is localized failure, the intact portion of the container still provides a barrier to release from the wasteform/container system. In this case, the release rates discussed above must be modified to take this into account. 
As water enters through the breached area, it might be stored within the container until a bathtub forms and the height of the bathtub reaches the lowest region of failure. At this time, water would begin to flow out of the container. Accurately predicting the location of failures around a container is beyond the state-of-the-art and will not be attempted here. Instead, it will be assumed that, once a container is breached, there will be steady flow of water into and out of the container. The container flow rate will be the Darcy velocity multiplied by the ratio of the breached area to the total area.

The partially-failed container will be treated as a mixing cell in which radionuclides released from the wasteform are uniformly mixed within the container. The release rate from the container will be the product of the container flow rate and the mixing cell concentration as calculated based on the various release mechanisms. This is identical to the approach used in the BLT computer code [Sullivan, 1989].

\subsubsection{Selection of Release Models}

As part of this program an evaluation of disposal data has been made [Cowgill, 1992a; Sullivan, 1991; Cowgill, 1992]. These reports discuss the distribution of radioactivity by waste class, waste stream, and wasteform and provide a starting point for selection of the appropriate leaching model.

A major finding of these studies is that in general, there is limited data on releases from most low-level waste streams or wasteforms. The lack of data covers the major waste streams: dry active wastes, dewatered resins, and activated metals. There is substantial data on releases of $\mathrm{Cs}, \mathrm{Sr}$, and $\mathrm{Co}$ in cement solidified wastes. Unfortunately, only a small fraction of the activity of these radionuclides is in cement. For radionuclides that do exhibit substantial quantities in cement, e.g., C-14, Tc-99, U-238, and Ra-226, there is also limited data.

Due to the lack of data, it is recommended that the surface rinse model be used for all dry active wastes and dewatered resins. If a partition coefficient is available and can be justified it should be used. For cement solidified wastes, leaching data indicate that diffusion controlled release should be used. The choice of diffusion coefficient should be supported by data. If data does not exist, a conservative estimate for the diffusion coefficient is $10^{-6} \mathrm{~cm}^{2} / \mathrm{s}$. This is equivalent to a Leach Index of 6 , the minimum allowed by the wasteform technical position [Lohaus, 1991]. For activated metals, a fractional release rate based on typical dissolution rates of the metals may be possible to justify.

For convenience, Table 2.1 presents a summary of the models selected for each of the four processes that influence release from the disposal facility. 


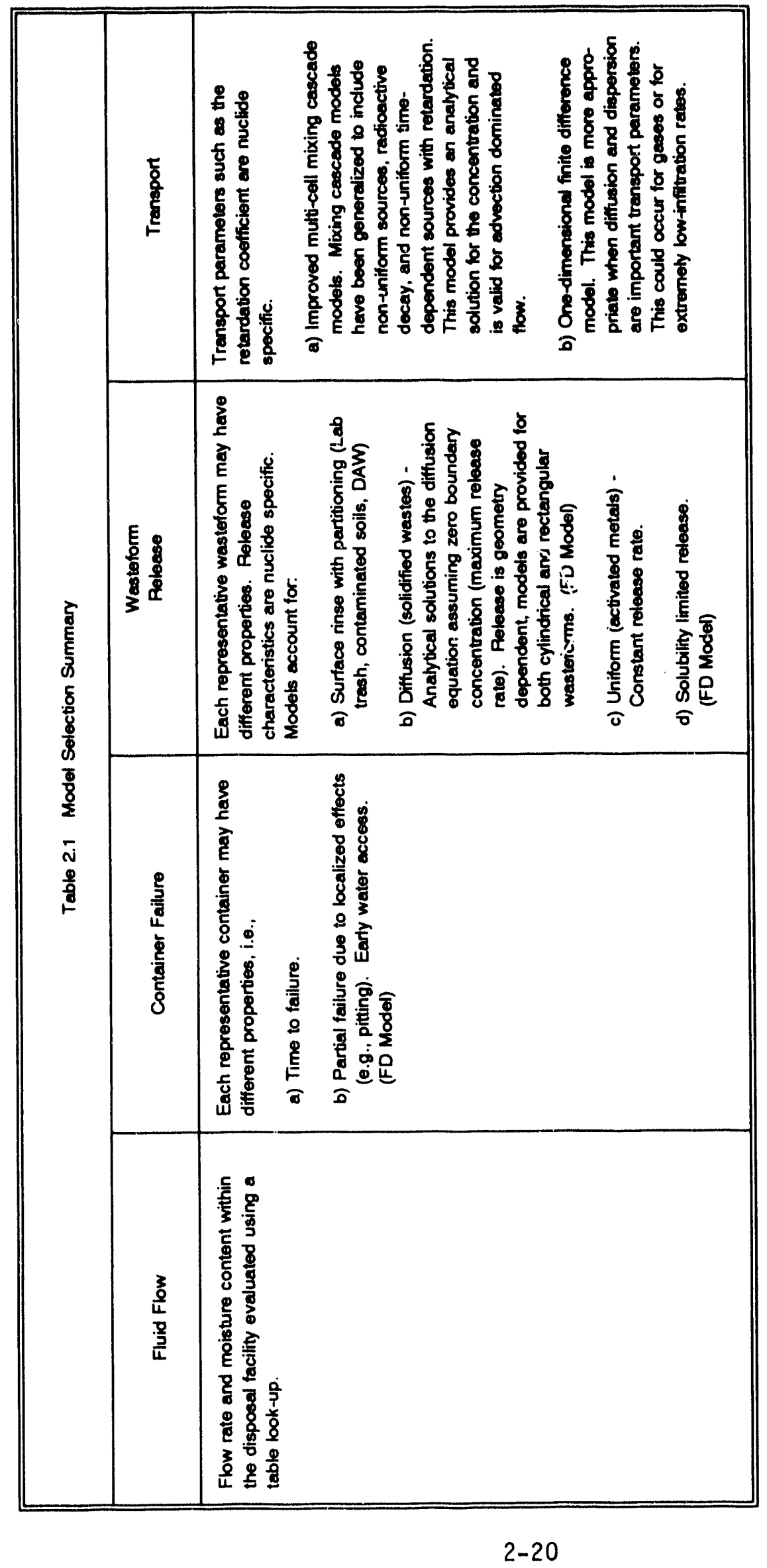




\subsection{Initial and Boundary Conditions}

In both the MCMC and FD transport models, the user must supply the initial concentration at every computational point.

In the MCMC model, boundary conditions are not necessary. The analytical solution assumes that the incoming concentration at the top boundary is zero. The bottom boundary condition is not needed due to the unidirectional flow and the absence of dispersion. That is, the concentration in the last node is independent of processes at the boundary.

In the FD model, both the top and bottom boundary conditions must be defined. One of four conditions are permitted to be specified at each boundary. These conditions are specified: concentration; total flux; advective flux; or dispersive flux. The description of the numerical implementation into the FD equations is presented in Appendix A.

In DUST, boundary conditions are defined through tabular input of the value for the boundary condition at a given time. Interpolation is used to determine the boundary condition value at times not in the table.

The boundary condition of zero concentration leads to the highest releases from the system. This condition implies that processes at the boundary are high enough to remove all material as soon as it exits the system.

The boundary condition of zero total flux prevents mass from leaving the system and causes concentration levels to be at a maximum. This condition is useful at a plane of symmetry or at the top boundary if zero release is desired. The total flux is the sum of the advective flux and the dispersive flux.

The advective flux is the Darcy velocity multiplied by the concentration. The Darcy velocity is known through input, therefore, specifying the advective flux is mathematically equivalent to specifying the concentration. The use of this condition is provided as a convenience to the user.

The dispersive flux is the diffusion/dispersion term multiplied by the concentration gradient. Setting this boundary condition to zero is useful when advection out of the system is the only mechanism for release, for example flow into a lysimeter drain. 


\section{PROCEDURE FOR CALCULATING RELEASES USING DUST}

The preceding chapter describes the models selected for estimating the source term but does not provide a clear indication of the steps needed to take the raw data, transform the data into the form required by the models, input the data and estimate the source term. Figure 3.1 is a schematic diagram that outlines this procedure.

The first step in this process is to compile the inventory data for the radionuclides of interest. Three factors that are determined before the waste is emplaced in a disposal facility figure prominently in determining release. These are the radionuclide's waste stream, wasteform, and container. A radionuclide contained in an activated metal will be released at a much different rate than the same radionuclide that exists as a surface contaminant on lab trash. Similar rernarks apply for releases from different wasteforms. The container will control the time that release begins and for localized failure, the amount of water that accesses the waste.

Work to determine the feasibility of characterizing the radionuclide inventory based on the three parameters listed above has been done. This effort, based on commercial disposal data from 1987 through 1989 [Roles, 1990], will determine the activity fraction of the waste streams contained in various wasteform and container types (e.g. HIC's, carbon steel, etc.). Discussion of the most important waste stream/wasteform/container systems in terms of activity are provided in [Sullivan, 1991b; Cowgill, 1992; Cowgill, 1992a].

Due to the large number of possible waste stream/wasteform/container combinations it will not be possible, nor even desirable, to model each of these systems individually. Therefore, many of the combinations that do occur should be lumped together to form a "representative" wasteform/container systems. This grouping should be performed to handle the most important wasteforms in terms of release. After the "representative" systems have been identified, appropriate container degradation and wasteform release models and parameters need to be determined.

Using the flexibility of the source term model, it will be possible to specify different release models and parameters for each waste stream/wasteform combination. For example, ion exchange resins solidified in cement may be assumed to follow diffusion-controlled release with one diffusion coefficient while evaporator bottoms solidified in cement may be given another, and activated metals may be assumed to follow dissolution controlled release with a constant release rate. Similarly, for two identical waste stream/wasteforms in two separate containers, the predicted release can be different due to different container properties.

The inventory, container degradation and wasteform release parameters are input into the source term model as schematically depicted in Fig 3.1. These parameters along with the radionuclide specific parameters (e.g. half-life, solubility limit, etc.), transport parameters, water f'ow parameters (velocity and moisture content), initial conditions, and boundary conditions (finite difference model only) fully describe the problem. 


\section{SOURCE TERM CALCULATION}

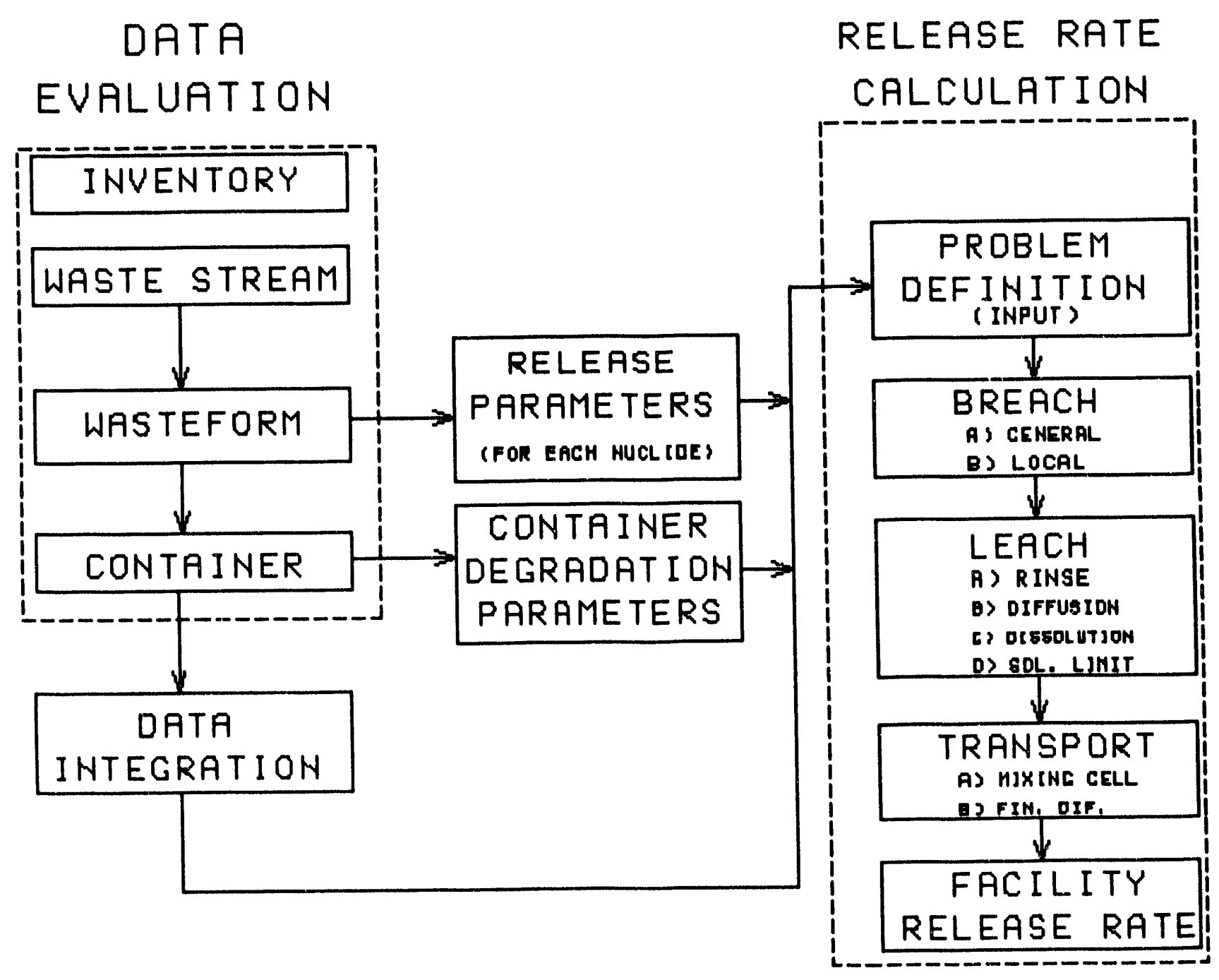

Figure 3.1 Flowchart of the procedure used to take waste stream/wasteform/container inventory data and define the necessary input parameters to estimate the release rate from a disposal facility. 
In general, the choice of input parameters used by the DUS'T code must be justified. Justification can come from experimental data, that is, use measured diffusion coefficients to predict wasteform release, from the use of more sophisticated computer codes, or welldocumented expert judgement. For example, the MCMC model in the DUST code assumes a constant flow rate through the disposal unit. In selecting the flow rate, a two-dimensional computer code that predicts unsaturated flow such as VAM2D could be used as a basis for determining this parameter. In any case documentation of the basis for the use of an input variable should be supplied with the resiilts of any simulation. The potential for misusing the simple models through improper choice of input data is large.

Once the data has been selected, an input deck for the DUST code must be created. To facilitate this process, the program DUSTIN was written. DUSTIN is a menu-driven code that prompts the user for the input required by DUST. DUSTIN allows the user to create an entire input deck or, modify an existing input deck. Modifications can be individually made to every single input parameter. Use of DUSTIN is described in Chapter 6.

After creating an input deck, the calculation is ready to proceed. For the MCMC model which relies on an analytical solution, the predicted release is calculated at the times specified through input. For the finite difference model, the predicted release is obtained through solving the differential equation describing release and transport through the disposal facility at a fixed time, incrementing the time and repeating the procedure until the problem is finished.

The output of these models will be the release rate from the disposal unit as a function of time. This output will be stored in tabular form for use with performance assessment codes, such as PAGAN [Chu, 1991], that predict the transport of radionuclides through the unsaturated zone to the aquifer and ultimately to a receptor.

If requested by the code user, DUST creates output files named TRACECND.DAT and TRACEFXD.DAT. These files contain the concentration at specified locations and the flux and mass release at specified locations as a function of time. If the FD transport model is used, the file CONCNT.DAT is written. This file contains the concentration at every location at the times requested in the primary output file. The program GRAFXT.EXE can plot each of these three files on a video display unit. The use of GRAFXT is described in Chapter 9. The procedure to be followed in creating an input deck, running the DUST code and analyzing the output is presented in Figure 3.2. 


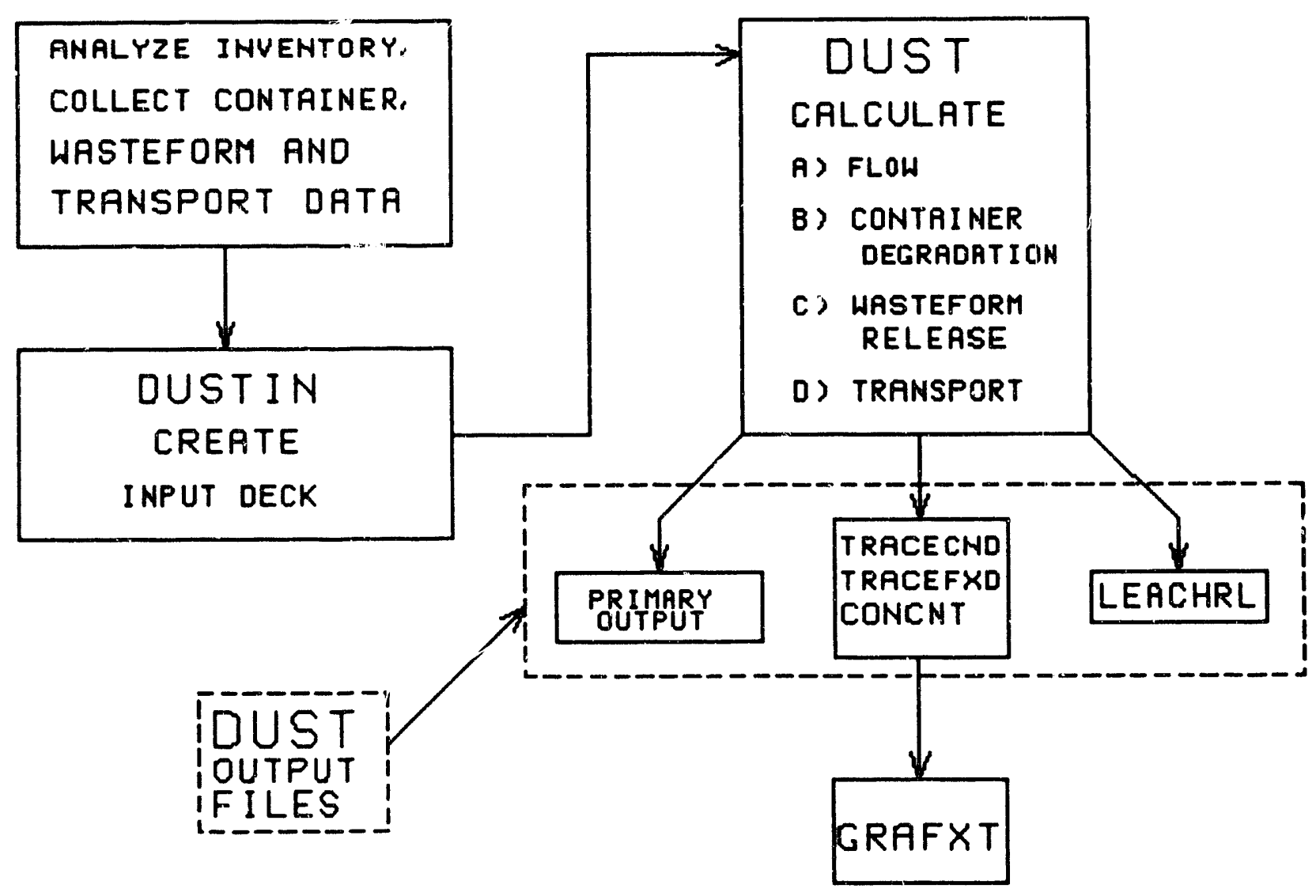

Figure 3.2 Schematic outline of the procedure used to analyze release from a LLW disposal facility using the DUST code package. 


\section{APPLICABILITY AND LIMITATIONS OF THE DUST CODE}

The DUST code models release and transport of a single radioactive contaminant through a low-level waste disposal facility in one spatial dimension. The source for transport is the contained radioactive wastes emplaced in the facility. Releases are a function of the container performance (time to failure) and wasteform performance (release rate). Each container may be assigned a unique time to failure and wasteform release rate parameters. This permits the flexibility to model a wide range of problems related to a waste disposal facility. These include determining the effects of the following parameters on release from the facility:
a) infiltration (flow rate);
b) container performance;
c) wasteform release rates; and
d) soil transport properties (retardation).

The DUST code can be applied to a wide range of problems pertaining to low-level waste disposal ranging from lysimeter studies in which there is only a single soil and no waste container to below ground vaults with caps, multiple containers and wasteforms, engineered structures and backfill. However, due to the simplifications used in developing the models, the validity of the predicted results depends quite heavily on the input data, as discussed in Chapter 3. Many parameters in DUST (for example, water flow rate and container failure time) are determined within the code directly from user supplied input and not from first principles. Therefore, justification of the choice of input parameters is a critical aspect in developing confidence in DUST predictions.

\subsection{Limitations}

In developing the DUST code, due to lack of mechanistic data and in order to limit the problem size to make the code executable on small desktop systems within a reasonable time, a number of assumptions were invoked. These assumptions may make use of the code inappropriate under certain conditions. In developing the $\mathrm{MCMC}$ model, a number of additional assumptions are used as compared to the FD model. The implication of these assumptions are discussed separately below. A list of the limitations follows.

(1) The DUST code simulates only one spatial dimension. It therefore assumes a uniform geometry in the other two dimensions. This approximation will be best near the center plane of the facility where edge effects are minimized. The lack of spatial resolution may cause problems near special features such as drains or fractures in engineering structures. Modeling the entire facility such that the bottom boundary has the properties of a drain will overestimate release, while modeling the facility as being completely intact may underestimate releases. 
(2) The DUST code models flow through a porous medium. Fracture flow, which may become significant at high relative moisture contents, through engineered barriers is not considered.

(3) Water flow and container failure time are not calculated from first principles in DUST. They are determined through input. The user must justify the values used for these parameters.

(4) DUST models only a single species. This influences three major areas: ingrowth due to radioactive decay, production or removal of species due to biodegradation, and interpreting solubility limits.

Ingrowth due to radioactive decay is not modeled. Ad hoc suggestions for modeling ingrowth were provided in the model selection report [Sullivan, 1991a]. For short lived radionuclides in secular equilibrium with the parent, it was recommended that a production term be added to the inventory. In the DUST code, this production term could be included in every container through use of an external source.

Biodegradation can produce radioactive gases, e.g. tritiated methane, ${ }^{14} \mathrm{CO}_{2}$, or ${ }^{14} \mathrm{CH}_{4}$. As a single species code, release in the gaseous and aqueous phase can not be handled simultaneously. An ad hoc procedure which partitions the inventory into the gaseous and aqueous phases may be used provided the code is run twice, once with the appropriate inventories and flow parameters for gaseous release and once for aqueous release. Work on estimating the partitioning between the gaseous and aqueous phases has been recently initiated.

In modeling only a single nuclide, care must be taken to insure that solubility limits are not exceeded if other species containing that element exist. The solubility limit used in the DUST code applies to the modeled nuclide only. If the element exists in more than one nuclide, the solubility limit must be decreased to account for this problem. For example, if the DUST code were used to model U-238 and solubility limits are used, this solubility limit should reflect the presence of other uranium species.

(5) DUST does not model changes in chemistry. The chemistry of the disposal facility is modeled through the distribution coefficient. Changes in this parameter due to changes in $\mathrm{pH}$, Eh and competition with other ions for sorption sites are not considered.

(6) In the FD model, the diffusion release subroutine is independent of the concentration in solution and solubility limits. This is a result of using the analytical solution based on a boundary condition of zero concentration at the wasteform-solute interface. 
(7) In the MCMC model, the Darcy velocity must remain constant at all times. In the FD model, the Darcy velocity is calculated from a table of velocity versus time.

(8) In the MCMC model localized container failure is not modeled.

(9) In the MCMC model the distribution coefficient and moisture content must be constant throughout the facility and $i_{i}$ time. The FD model permits these parameters to vary spatially.

(10) In the MCMC model release mechanisms are limited to the uniform release and rinse release without a wasteform partitioning factor. The FD model allows a partitioning factor and models diffusion controlled release.

(11) As shown in the development of the equations, both the MCMC and FD models use upwind differencing to model the advection term. Upwind differencing insures that information is only advected downstream, however, it leads to numerical dispersion. The value of the numerical dispersion coefficient, $D_{n}$, in the FD model is [Roache, 1976]:

$$
D_{n}=\frac{V_{d} \Delta x}{2}(1-C)
$$

where $V_{d}$ is the Darcy velocity, $\Delta x$ is the size of the finite difference node, $C$ is the dimensionless Courant number, $V_{d} \Delta t / \Delta x$, and $\Delta t$ is the time step size. In the limit as the time step size approaches 0 , the solution to the FD equation approaches the solution of the original differential equation modified due to numerical dispersion. In this case, the Courant number goes to zero and the numerical dispersion coefficient becomes:

$$
D_{n}=\frac{V_{d} \Delta x}{2}
$$

Heuristically, this can be viewed as the numerical dispersion coefficient for the MCMC model which uses an analytical solution. Numerical studies comparing the FD model with zero mechanical dispersion and diffusion with the MCMC model support this heuristic estimate of numerical dispersion for the MCMC model. 
Recalling that the mechanical dispersion term from Eqn (2.1) is:

$$
D=V_{d} a_{t},
$$

it is seen that the numerical dispersivity is $\Delta x / 2$. Therefore, if the mesh size is more than twice as large is the mechanical dispersivity value, numerical dispersion will dominate. From the numerical dispersion expression it is clear that it can be minimized through taking small nodes. As a rule of thumb, the mechanical dispersion is typically $1 / 10$ to $1 / 100$ of the scale of the modeled domain. Therefore, $\Delta \mathbf{x}$ should be much smaller than $1 / 5$ to $1 / 50$ of the modeled domain to insure that numerical dispersion is unimportant.

(12) Although conceptually, modeling gas flow through the facility is similar to modeling water flow, there is extremely little data on production of radioactive gases or expected flow rates. Therefore, the user must be extremely careful when selecting these values.

Radionuclides disposed of in gaseous form are expected to release quickly after container failure. For adsorbing gases it is expected that release would be controlled by a partitioning factor. Gases can also be formed by biodegradation, for example, tritiated methane, ${ }^{14} \mathrm{CO}_{2}$, or in the case of radon, through radioactive decay of radium. Few data exists on the formation of radioactive gases in a disposal facility. However, tritiated methane, ${ }^{14} \mathrm{CO}_{2},{ }^{14} \mathrm{CO}$, and other radioactive gases have been detected at the closed disposal sites at Sheffield [Streigel, 1985] and West Valley [Kunz, 1982; Matuszek, 1983]. While it is likely that the better disposal techniques (concrete vaults, no wooden or cardboard containers, solidification of the wastes in cement, etc.) planned for the new facilities may lead to less organic material, many of the wastes contain significant amounts of organics. This is particularly true for ${ }^{14} \mathrm{C}$ wastes [Gruhlke, 1986]. Therefore, releases caused by biodegradation cannot be dismissed at this time.

As a final caveat, uncertainties in the input data are often the most significant limitation in many models and codes. Frequently, many input parameters are not accurately known or available. Users of the DUST code should be constantly aware of the limitation imposed by the quality of the input data. 


\section{VERIFICATION TESTS}

A number of studies have been conducted to verify that the computer code, DUST, correctly calculates the properties of interest (time of container breach, wasteform release rates, solution concentration, and mass flux). Independent testing of the container degradation models (general and local failure) and the wasteform release models (diffusion, uniform, surface rinse with partitioning, and solubility limited) was performed for problems with known analytical solutions.

The FD and MCMC transport models received extensive testing through comparison of predicted results to analytical solutions and through comparison of the MCMC results and FD results on identical problems.

For verification testing, Eqn (2.3) has been solved for a semi-infinite medium and the following initial and boundary conditions:

$$
C(x, 0)=C_{i}
$$

The boundary condition at $\mathrm{x}=0$ is:

$$
\left(-\theta D \frac{\partial C}{\partial x}+V_{d} C\right)=\left\{\begin{array}{lr}
V_{d} C & 0<t \leq t_{0} \\
0 & t>t_{0}
\end{array}\right.
$$

where $\mathrm{C}_{\mathrm{o}}$ and $\mathrm{C}_{\mathrm{i}}$ are constant. The analytical solution to this problem is presented in [Van Genuchten, 1978].

The analytical solution requires a non-zero value for the diffusion/dispersion term. Therefore, the FD model was tested against the analytical solution for three problems. In these problems, advection, dispersion, retardation, and decay are modeled. Three test cases were run. In these cases, the parameters chosen for the model were selected to correspond to the values used by Van Genuchten and can be found in Table 5.1. 


\begin{tabular}{||l|l|l|l||}
\hline \multicolumn{4}{|c|}{ Table 5.1 Parameters used to verify the finite difference transport model } \\
\hline & Case 1 & Case 2 & Case 3 \\
\hline & & & \\
Darcy Velocity $(\mathrm{cm} / \mathrm{s})$ & $2.89 \mathrm{E}-4$ & $2.89 \mathrm{E}-4$ & $2.89 \mathrm{E}-4$ \\
Dispersion/Diffusion $\left(\mathrm{cm}^{2} / \mathrm{s}\right)$ & $4.34 \mathrm{E}-4$ & $4.34 \mathrm{E}-4$ & $4.34 \mathrm{E}-4$ \\
Decay constant $(1 / \mathrm{s})$ & $2.51 \mathrm{E}-6$ & 0.0 & $2.51 \mathrm{E}-6$ \\
Source $\left(\right.$ curies $/\left(\mathrm{cm}^{3}\right.$-s) $)$ & 0.0 & 0.0 & $1.16 \mathrm{E}-5$ \\
Moisture content & 0.3 & 0.3 & 0.3 \\
Retardation coefficient & 3.3 & 3.3 & 3.3 \\
& & & \\
\hline
\end{tabular}

The semi-infinite domain was modeled using $300 \mathrm{~cm}$. and comparing the semi-infinite analytical solution to the numerical solution over the first $100 \mathrm{~cm}$. for the first 10 days. Numerical studies were performed with the DUST code to show that, for this time frame and distance, the boundary conditions at the $300 \mathrm{~cm}$. boundary do not influence the results.

In the first problem, case 1 , solute was injected into the boundary at $x=0$ for 5 days such that the incoming concentration was $1 \mathrm{Ci} / \mathrm{cm}^{3}$. After the fifth day, the flux at $x=0$ is zero. At the boundary away from the injection source, the boundary concentration is set to zero. The initial condition is zero concentration throughout the modeled domain. Advection, dispersion, retardation and radioactive decay were modeled.

Figure 5.1 presents a comparison of the analytical and numerical solution predicted by the DUST code at 5 and 10 days. The agreement between the two is excellent. The maximum difference between the two solutions is less than $2 \%$.

Test case 2 was identical to case 1 with the exception that the decay term was set to zero. This is a slightly more difficult numerical problem because the concentration gradients are larger than in case 1. Again, the agreement between the analytical and numerical solutions at 5 and 10 days is excellent, Figure 5.2.

Test case 3 has the same transport properties as test case 1, Table 5.1, however, the problem begins with a uniform initial concentration of $10 \mathrm{Ci} / \mathrm{cm}^{3}$, a uniform source of $1.16 \mathrm{E}-5$ $\mathrm{Ci} / \mathrm{cm}^{3} / \mathrm{s}$, and zero total flux at the $\mathrm{x}=0$ boundary. This problem tests the proper use of the external source (i.e., wasteform release) term and use of non-zero initial conditions.

Figure 5.3 contains a plot of concentration versus distance at 2,5 , and 10 days. The results indicate that the DUST code is capable of reproducing the analytical solution with a high degree of accuracy. 


\section{Comparison of DUST Predictions with analytical solution}

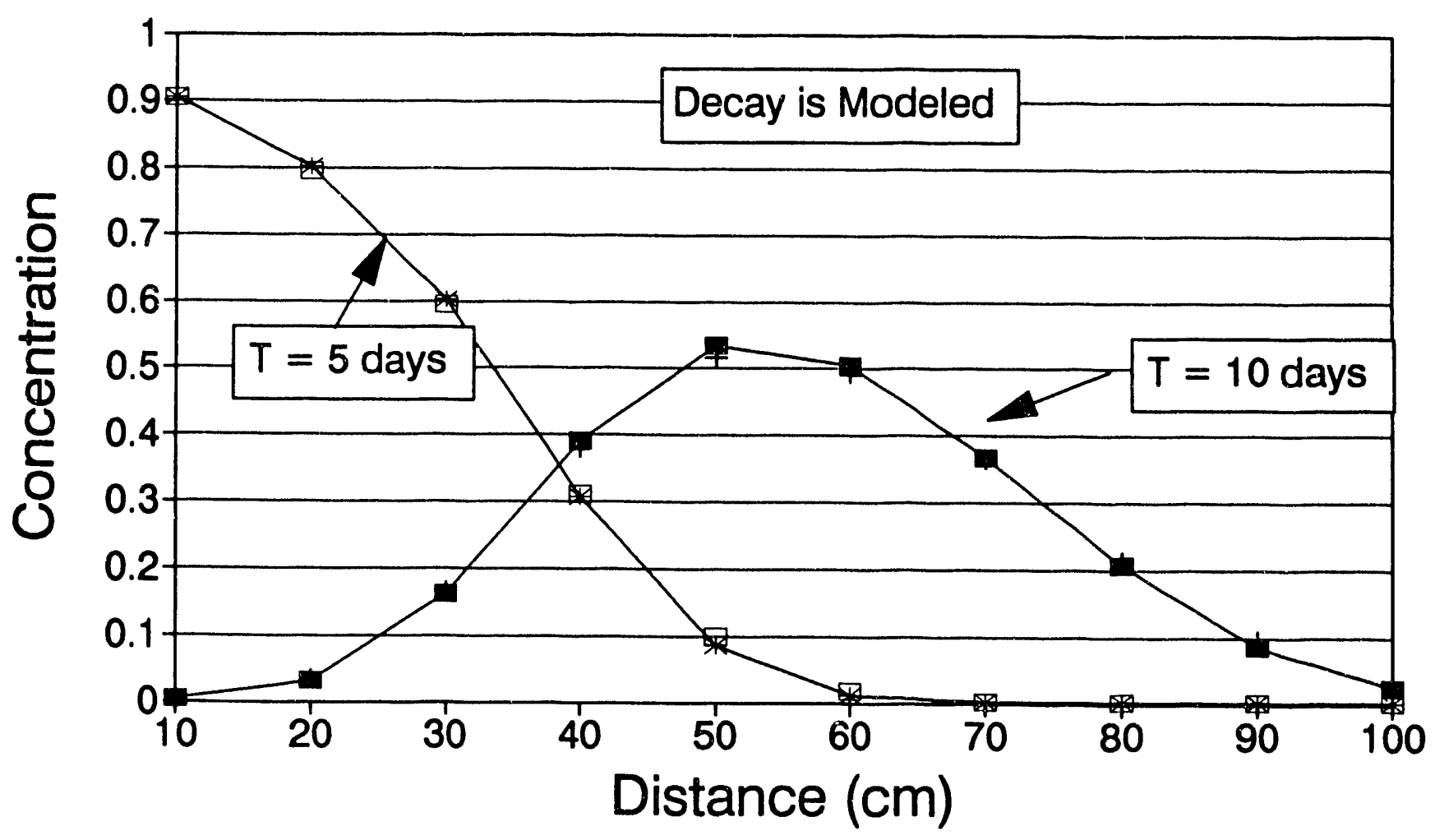

$\rightarrow-t=10 d+t=10 d-$ DUST $\rightarrow t=5 d \quad \square \quad t=5 d-$ DUST

Figure 5.1 Test case 1, comparison of the DUST finite difference model predictions with the analytical solution for a pulse source at $\mathrm{x}=0$ for 5 days. Retardation and decay are modeled. See Table 5.1 for the model parameters. 


\section{Comparison of DUST Predictions with the Analytical Solution}

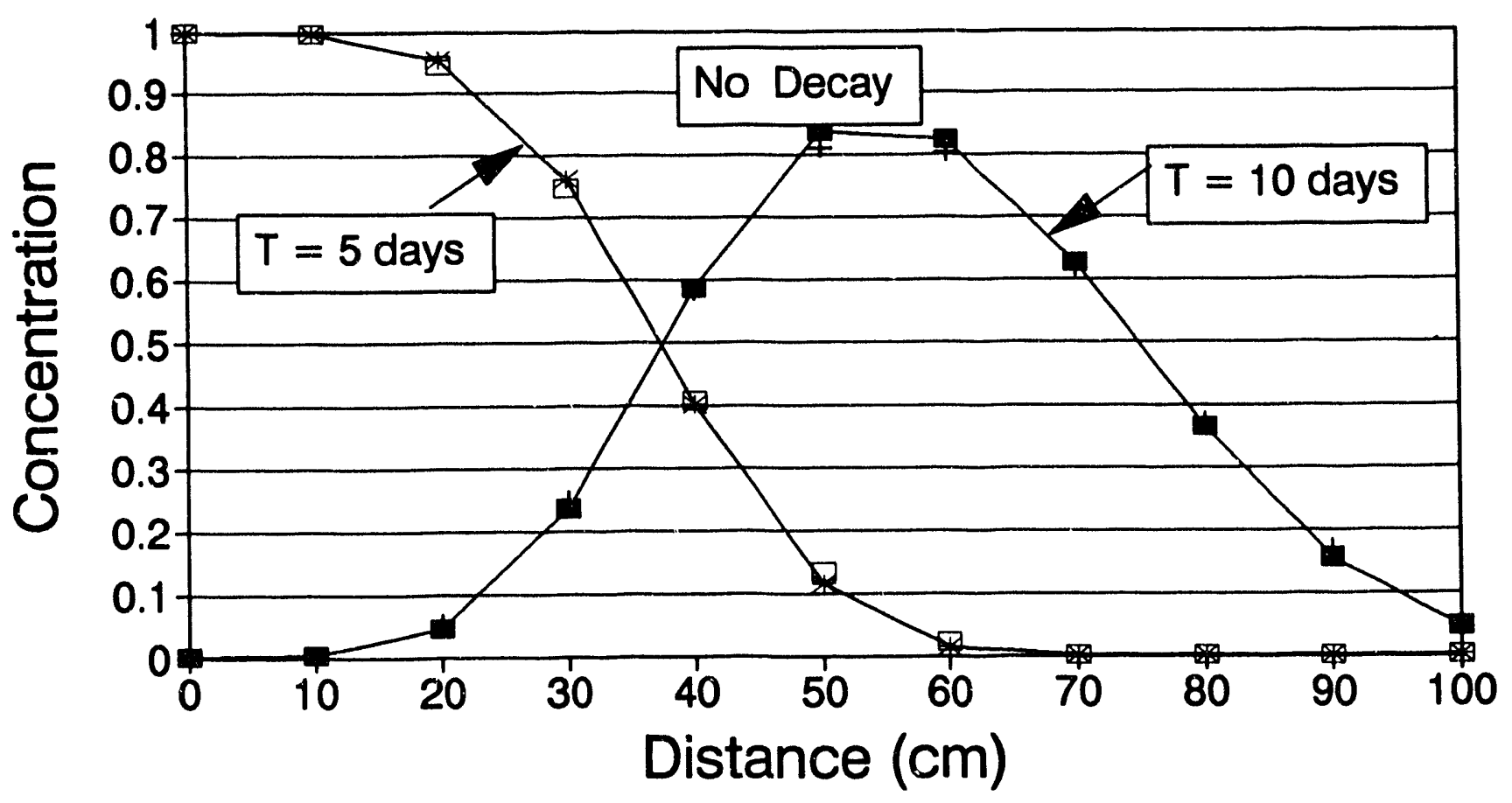

$\rightarrow-t=10 d+t=10 d-$ DUST $\rightarrow-t=5 d \quad \square t=5 d-$ DUST

Figure 5.2 Test case 2, comparison of the DUST finite difference model predictions with the analytical solution for a pulse source at $x=0$ for 5 days. The decay term is zero in this simulation. See Table 5.1 for the model parameters. 


\section{DUST Predictions versus Analytical Solutions}

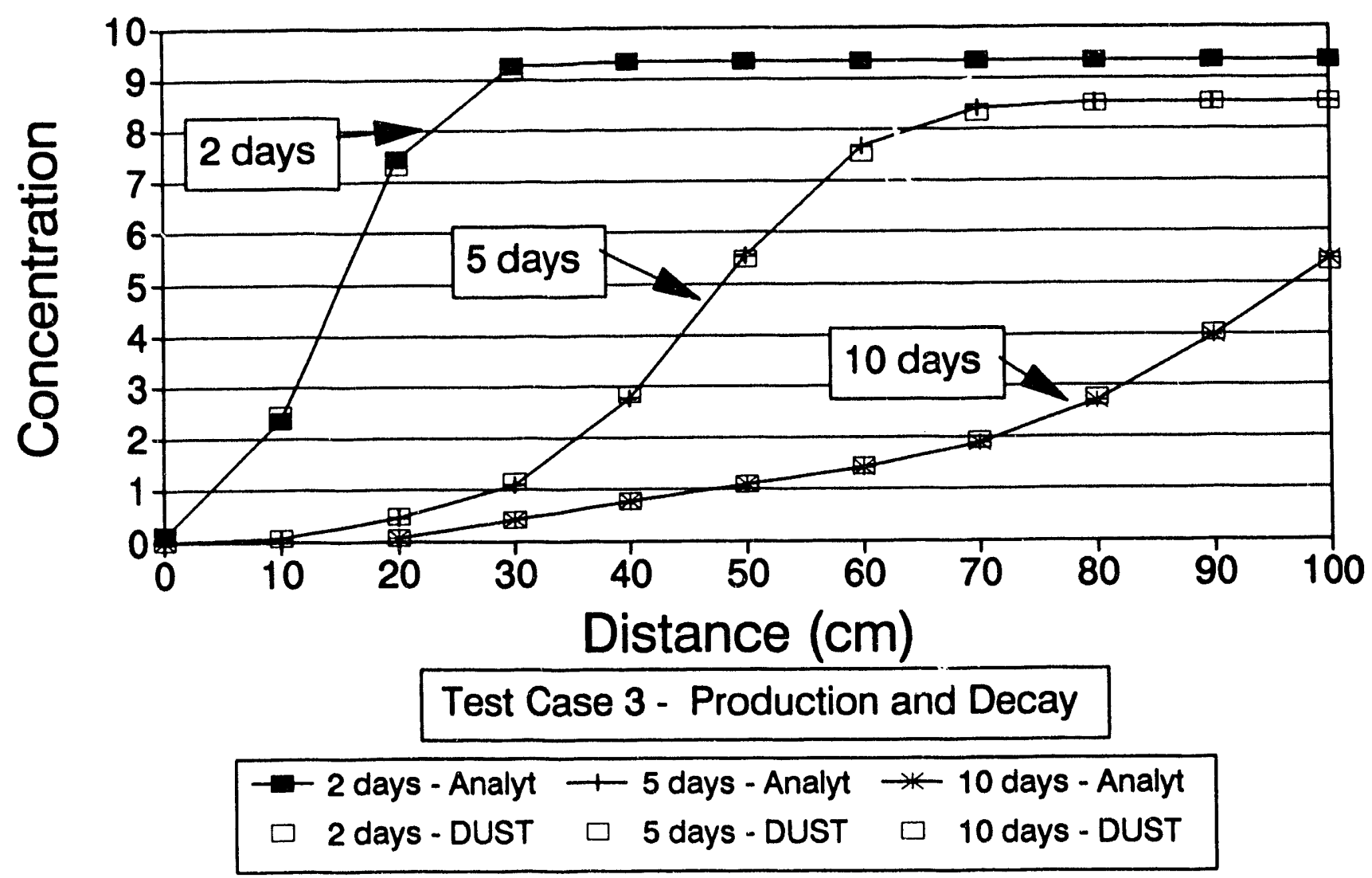

Figure 5.3 Test case 3, comparison of the DUST finite difference model predictions with the analytical solution for a uniform initial concentration, zero total flux at the boundary $\mathrm{x}=0$, and an external uniform source. See Table 5.1 for the model parameters. 
In the preceding problems, diffusion/dispersion plays an important role in the transport of the radionuclide and therefore, the analytical mixing-cell cascade model cannot be used. The mixing-cell cascade model was compared directly with hand calculations of the analytical solution [Appendix A] for several simple problems (few cells, all containers fail simultaneously, constant release rates). In addition, a number of test cases were run in which the diffusion/dispersion term was set to zero in the FD model. This allowed comparison of the predictions of the FD and MCMC models directly.

Test case 4 is an example of one of these problems. In test case 4, the domain for the finite difference model is $\mathbf{5 0}$ meters. For this simulation, the domain was subdivided into 50 regions, each 1 meter in length. The first 10 meters contain only soil. In the next 24 meters there is a wasteform every other meter for a total of twelve wasteforms. The container failure times range from 0 to 40 years as presented in Table 5.2. Release from the wasteforms is modeled using the uniform release model. The parameters for this model were chosen such that once water contacts the wasteform the fractional release rate is $5 \%$ per year, all other parameters are presented in Table 5.2. The problem considers radioactive decay, retardation, and advection.

\begin{tabular}{|c|c|c|c|c|c|c|c|c|c|c|c|c|}
\hline Table 5.2 & \multicolumn{6}{|c|}{$\begin{array}{l}\text { Parameters used in test case } 4 \text { : } \\
\text { finite difference model results }\end{array}$} & \multicolumn{6}{|c|}{ Comparison of the mixing cell and } \\
\hline \multicolumn{13}{|c|}{ Container failure times: } \\
\hline Location (m) & 1 & 3 & 5 & 7 & 9 & 11 & 13 & 15 & 17 & 19 & 21 & 23 \\
\hline Time (yrs) & 0 & 10 & 20 & 30 & 40 & 0 & 10 & 10 & 20 & 20 & 0 & 30 \\
\hline \multicolumn{13}{|c|}{ Release and Transport Parameters: } \\
\hline \multicolumn{5}{|c|}{ Darcy Velocity $(\mathrm{cm} / \mathrm{s}):$} & & $1.59 \mathrm{I}$ & & & & & & \\
\hline \multicolumn{5}{|c|}{ Moisture Content: } & & 0.2 & & & & & & \\
\hline \multicolumn{5}{|c|}{ Retardation coefficient } & & 9.0 & & & & & & \\
\hline \multicolumn{5}{|c|}{ Release rate $(1 / \mathrm{yr})$} & & 0.05 & & & & & & \\
\hline \multicolumn{5}{|c|}{ Half-Life (yrs) } & & 12.33 & & & & & & \\
\hline
\end{tabular}

The mixing cell simulation was identical to the finite difference model with the exception that only 40 meters was modeled. For advection driven flow, the 10 meters upstream from the wasteform do not receive any contaminant. Thus, the two test cases are identical except that the distance from the top of the simulation domain is offset by 10 meters. The difference is necessary to insure that the boundary condition of the finite difference solution does not influence the results. 
Figure 5.4 presents a comparison of the results of the two simulation techniques at three different locations for a 90 year period. Location 1 is 7 meters from the top of the first wasteform, this cell has a container that fails after 30 years. Location 2 is 12 meters from the top of the first wasteform. It does not contain a wasteform. However, the adjacent upstream cell has a waste container that fails instantly. Location 3 is 23 meters from the top of the first wasteform. It has a container that fails after 30 years. Two meters upstream from this wasteform is a waste container that fails instantly.

In all locations and at all times, the mixing-cell model and the finite difference model show excellent agreement. At location $1, x=7 \mathrm{~m}$ in Fig. 5.4, there is a gradual buildup of the concentration in time due to container failures upstream. At 30 years, there is a slight jump in concentration due to the failure of the container in the cell. At location $2, x=12 \mathrm{~m}$ in Fig. 5.4, there is a rapid rise in concentration at early times due to the failure of the container 1 meter upstream. The concentration peaks after about 10 years and begins to decline for approximately 30 years. At this time, other containers upstream have failed and begun to release contaminants. The concentration shows a local maximum after about 50 years as the containers 3 and 5 meters upstream failed after 40 and 30 years, respectively. At location $3, x=23 \mathrm{~m}$ in Fig. 5.4, there is a peak after 12 years due to failure of the container $2 \mathrm{~m}$ upstream at emplacement. The concentration decreases until 30 years where a sharp increase occurs due to the failure of the container in this cell. 


\section{Comparison of MCMC and FD Models Multiple Container Fallure Times}

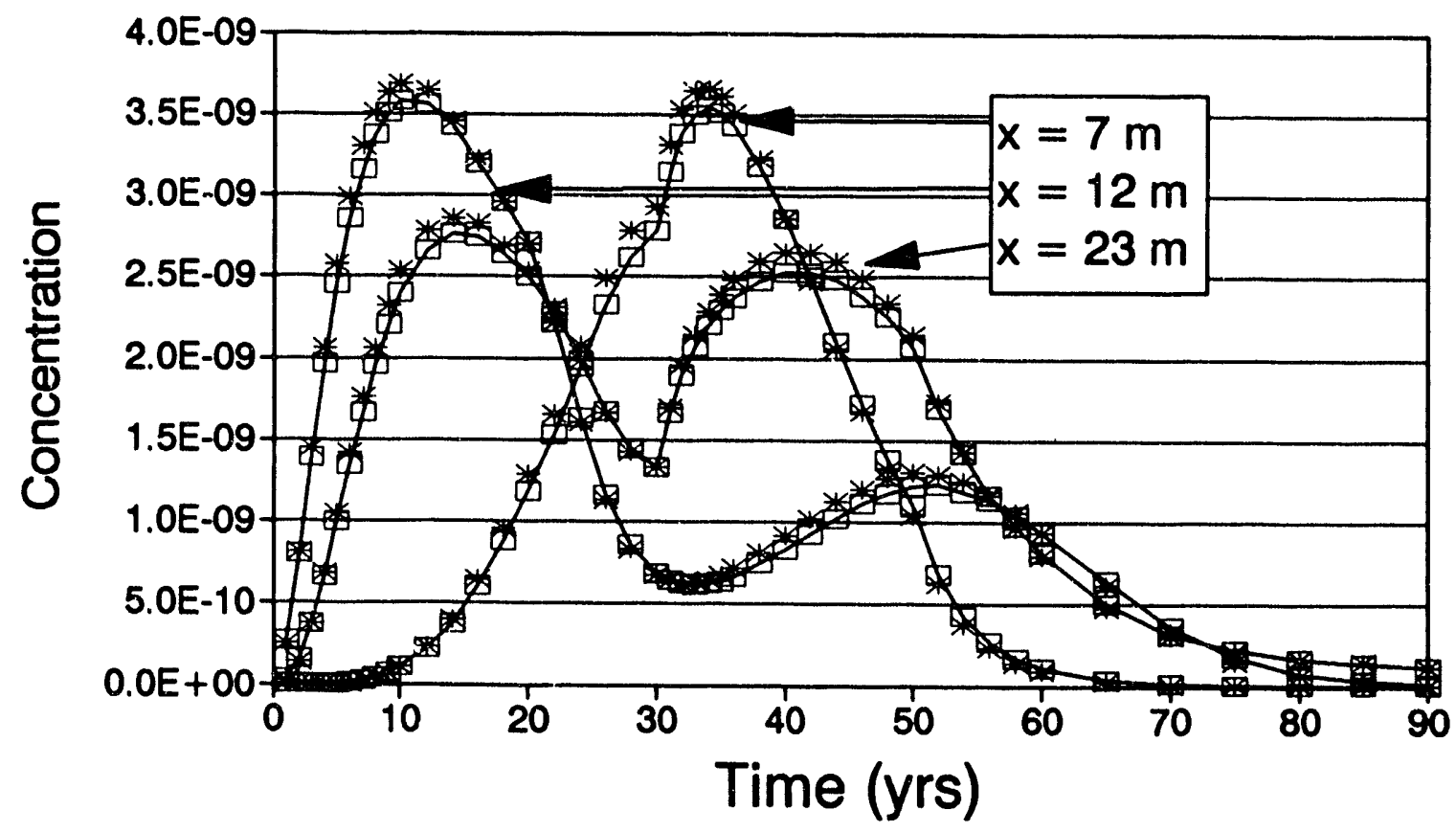

$\varpi$ MCMC $\quad$ * Finite Difference

Figure 5.4 Test case 4, comparison of the DUST finite difference and mixing cell cascade model at three locations. There are 12 wasteforms, each one meter in length separated by one meter of soil beginning at $x=0$. The container failure time differs for the wasteforms as described in Table 5.2. Release and transport parameters can also be found in Table 5.2 


\section{DUSTIN: A PREPROCESSOR THAT CREATES AN INPUT DECK FOR DUST}

In order to facilitate ease of use of the DUST computer code, a pre-processor which takes the user through all of the steps necessary to create an input deck has been written. This preprocessor, DUSTIN, relieves the code user of knowing the exact format and structure of an input deck and is menu driven. The menus present a series of choices and generally request a numeric response. In the few cases when an alpha-numeric response is required, e.g., defining titles, this is clearly noted.

DUSTIN has the flexibility to independently alter any sing!e parameter required by the DUST code. DUSTIN has the capability of creating an entirely new input deck or reading a partially or fully completed input deck which can then be modified. A major advantage in using the DUSTIN code is that it provides an annotated input file for DUST. This greatly facilitates direct modification of the input deck, Chapter 7.

The first menu asks the user if a completely new input deck is to be created or if an existing input deck is to be modified. After this decision has been made, the code proceeds to the main menu. The main menu permits the user to modify any variable required for input to the DUST code independently through access to sub-menus. After the input variables have been defined, DUSTIN allows the user to create a trial input deck or create a "partial" input deck. The flexibility allowed in being able to independently modify any single variable within the code makes it nearly impossible to guarantee that a consistent input deck is created by the DUSTIN user. Although, there are many checks within the code to prevent obvious problems, it is possible to create an invalid input deck. For example, the user could define the number of containers to be 20 and not specify any failure parameters for the containers. If this occurs, DUSTIN will try to make an input deck if requested, but obviously the input deck will not be valid. For this reason, DUSTIN permits the user to create an output file that is identical to the output file that would be obtained if the DUST code were used. This is a useful aid in debugging the trial input deck.

In addition, DUSTIN permits the user to create a "partial" input deck which can be read in later by the DUSTIN code and modified as necessary. Use of this feature is strongly recommended. A "partial" input deck is any set of input data created by DUSTIN using the namelist procedure described later in this section. The advantage to this approach is that the check to determine if the data forms a valid input file is not made when reading the data. In contrast, if the user attempts to read in a completed input deck that is not valid, an error message is printed and control is returned to the operating system. That is, data on an invalid completed input deck cannot he modified by DUSTIN.

The remainder of this chapter takes the reader through the various menus that appear when running the DUSTIN computer code. All sections that have a double border and are highlighted in boldface print are the screens that appear on the console. Italic characters are examples of the code users response to the query. For convenience in referencing and to enhance the ease of locating discussions of the parameters, the numbering system for this chapter will correlate to the 
nuinbers in the main and secondary menus. For example, Section 6.4 .3 will refer to the fourth item on the main menu and the third item on the secondary menu. For this reason, the input selections menu and the main menu will appear in this section.

In general, far fewer variables are needed to use the Multi-cell Mixing Cascade (MCMC) transport model as compared to the Finite Difference (FD) transport model. These differences are explicitly and piominently mentioned in the DUSTIN ode. For example, if the user attempts to define a variable that is not required by the MCMC model, the DUSTIN codle will print a message indicating such. If a variable is required by only one model, the designator FD or MCMC will be displayed at the top of the section describing the variable.

This chapter will serve as the most detailed reference on the input variables required for DUST. In addition to describing the operation of the DUSTIN code, selection of the appropriate values for the physical parameters, instructions on the operations of the models, and recommendations on when to use certain models will be primarily provided in this chapter.

INPUT SELECTIONS:

When running the DUSTIN code, the first menu is:

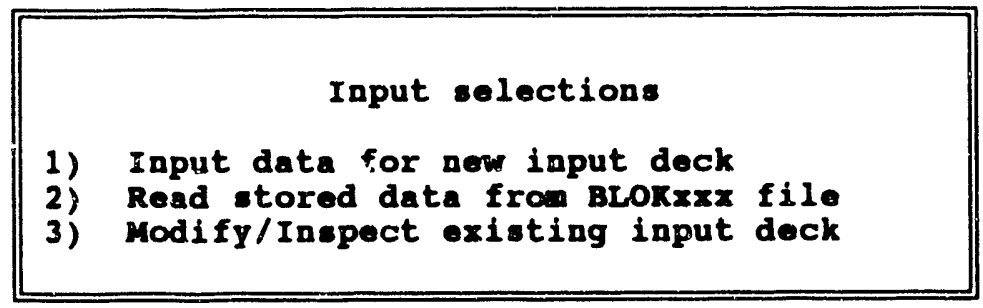

Choice $=1:$

The code user $w$ :Il create an entirely new input deck. Default values are specified for some parameters but generally these need to be redefined. After choosing this value, the main menu is displayed.

Choice $=2$ :

Upon entering a value of 2 , the following screen is displayed: 


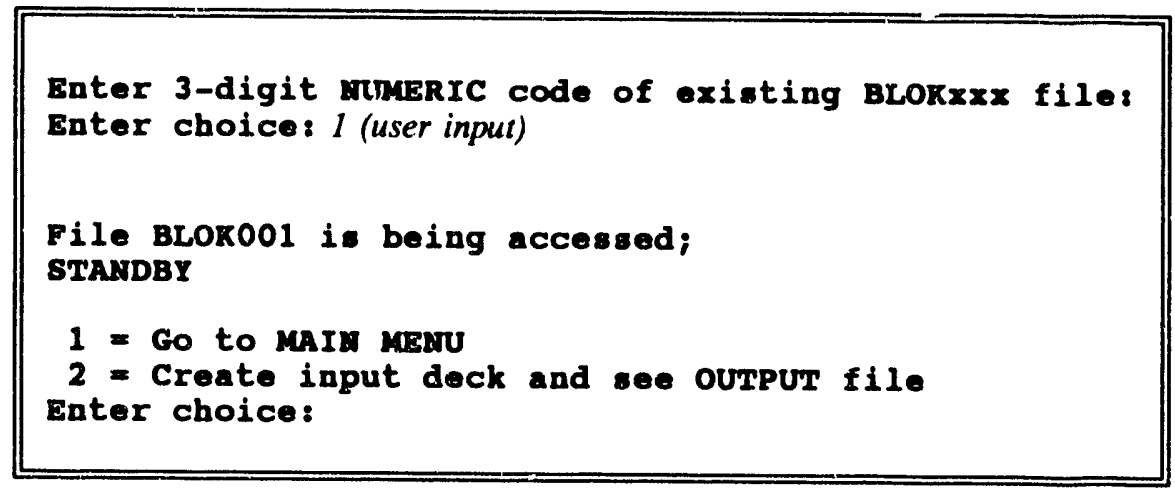

DUSTIN will read a file previously prepared by DUSTIN called BLOKxxx, where $\mathbf{x x x}$ is a three digit number. In the example, the 1 digit number is translated by the code to 001 and the file BLOK001 is read. BLOKxxx is a file created by using FORTRAN namelists. As such, it is an exact copy of all the input variables needed by the DUST code at the time that the file was written. There are no checks on whether this set of variables forms a valid input deck. Therefore, the BLOKxxx file is useful when the code user is uncertain if a valid input deck has been created or if a partial input deck has been created.

The code checks to determine if the BLOKxxx file exists. If it does not exist, the user is returned to the Input Selections Menu. After successfully reading the file, the code user is allowed to attempt to make an input deck or proceed to the main menu.

Choice $=3$ :

The code requests the name of the input file, a name for the output file to be created, and if the output file exists, the code asks if the file should be overwritten. The completed screen for this procedure is displayed:

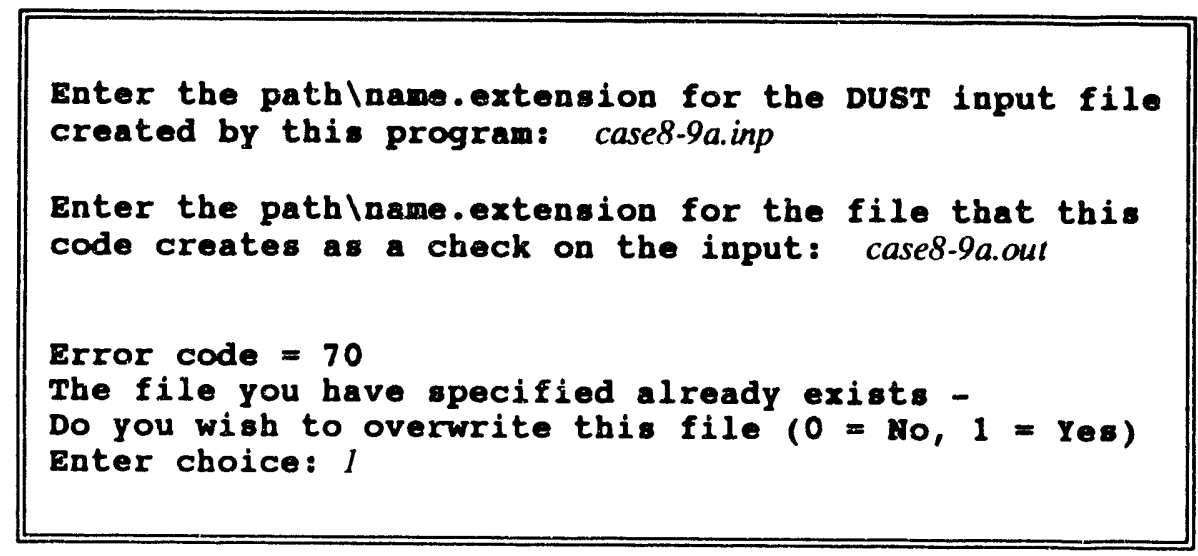


The DUSTIN code then proceeds to the Main Menu. If the file case8-9a.inp did not exist or if the file does not contain a valid input deck, the code fails and control is returned to the operating system.

\section{MAIN MENU:}

The main menu consists of a list of general categories that comprise the groups of input parameters needed by the DUST code. The main menu provides access to the sub-menus defined by the grouping scheme. Definition of the input variables occurs in the sub-menus. This section will provide a description of the main menu and the variables contained under the grouping scheme. Detailed discussions on the variables and how they impinge on model predictions will be presented when discussing the sub-menus. The main menu for DUSTIN follows:

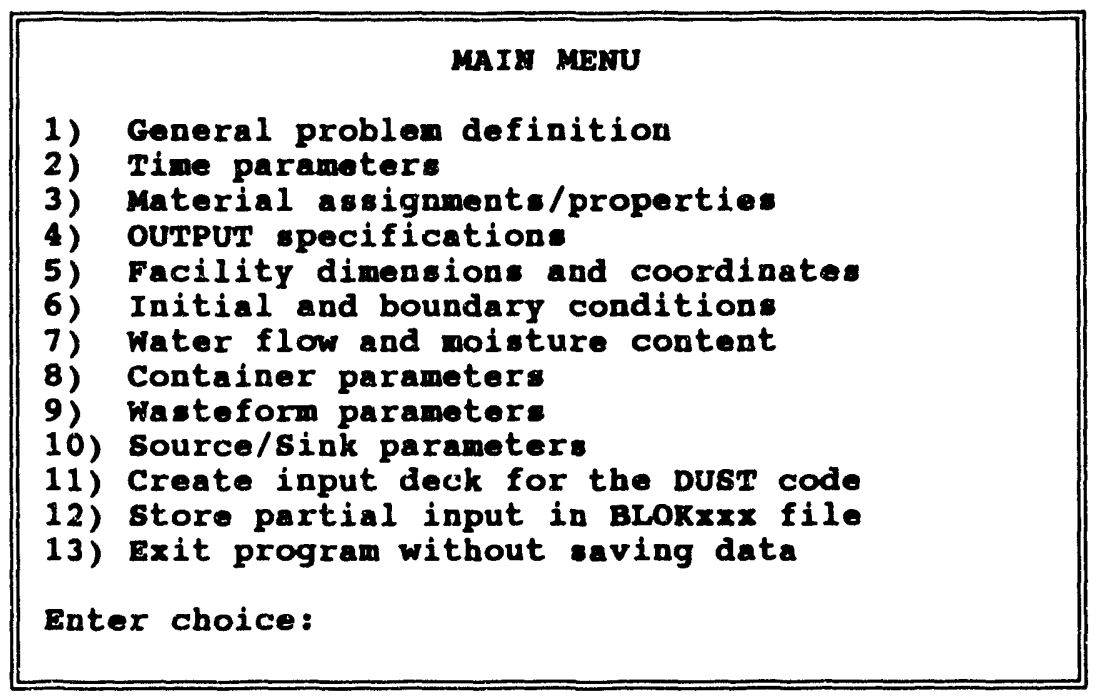

Choice $=1:$ General problem definition

The general problem definition includes variables which define the following: title, name of the radionuclide, half-life, atomic mass, solubility limit, a flag to specify if the input is in mass units of grams or curies (if the input is in curies, the code internally translates this to grams for consistency with the units on the distribution coefficient and solubility limits, upon output, the mass is translated back into the original input units), number of control volumes or mixing cells in the computation, and the flag for selecting the multi-cell mixing cascade or the finite difference transport model.

Choice $=2:$ Time parameters

Time parameters include the number of output times and their values for the MCMC transport model and the number of time steps, the number of time step changes, the ir itial time 
step, fractional change in time step, maximum time step, and maximum problem time for the FD transport model.

Choice $=3:$ Material assignments/properties

Material properties for the MCMC model include the bulk density and soil distribution coefficient. The MCMC allows only one material type. The FD allows multiple material types and requires the number of materials, the material type for each control volume, the bulk density and the soil distribution, dispersion, and diffusion coefficients.

Choice $=4:$ OUTPUT specifications

Output specifications include printer control variables which determine if output occurs at each time step, the number and location of concentration and flux traces, and the number of time steps between writing values to the trace files.

Choice $=5:$ Facility dimensions and coordinates

Facility dimensions and nodal coordinates include the surface area and height of the facility for the MCMC transport model. The MCMC model assumes uniform spacing and, therefore, the thickness of each cell is the height of the facility divided by the number of cells. The FD transport model allows variable thickness cells and, therefore, the thickness of each cell must be specified along with the facility surface area. The height of the facility is calculated from the input when the FD transport model is specified. The surface area of the facility is a normalizing factor to account for the volume of the 3-D facility in a 1-D model. It is an important parameter in determining concentrations of radionuclides in solution. For example, if the mass inventory is released instantly and uniformly across the facility, the concentration is determined from the inventory, height, surface area, and moisture content. Since dose is proportional to concentration, the normalization by surface area is required.

Choice $=6:$ Initial and boundary conditions

Initial and boundary conditions are required for the FD model. The boundary conditions for the MCMC model are fixed (zero incoming concentration) by the analytical solution of the model and are not required. The FD model allows specified concentration, total flux, advective flux, or dispersive flux as a boundary condition.

Choice $=7:$ Water flow and moisture content

The Darcy velocity and moisture content are required by both transport models. The MCMC model permits only a time-invariant uniform Darcy velocity and moisture content in the modeled domain. The FD model permits a spatially uniform, time-varying Darcy velocity, and a spatially varying, time invariant moisture content. A time varying Darcy velocity could be used to model degradation in the ability of the cap to prevent water ingress. 
Choice $=8:$ Container parameters

Container parameters in the MCMC model include the number of containers, their location in the modeled domain and their failure time. In addition, the FD model allows localized (pitting) failure and, therefore, requires information on the localized failure rate parameters as well as assignment of these parameters to each container.

Choice $=9:$ Wasteform parameters

Wasteform release parameters include the inventory, the number of different sets of release rate parameters, a flag to indicate which release rate parameters are to be used on each waste form, and the release rate parameters. The MCMC model permits only rinse release subject to partitioning and an exponentially decaying release rate where the exponent accounts for radioactive decay. In addition, the $F D$ model permits diffusion controlled release from either cylindrical or rectangular finite sized waste forms. Therefore, input is required to define the diffusion coefficients and the dimensions of the waste form.

Choice $=10:$ Source/Sink parameters $($ FD Model Only)

In addition to the waste forms releasing contaminants into the system, external sources or sinks can be specified by the code user if the FD transport model is specified. These sources are defined through tabular input which provides the source strength as a function of time, as well as input specifying the number of sources, the number of different types of sources (e.g., number of source strength versus time tables), the location of the sources, and the assignment of a source type to a location.

Choice $=11:$ Create input deck for the DUST code

This selection creates the input deck to be used by the computer code DUST. It first asks for the file name for the input file. If it exists, it asks the user if it should overwrite this file. It then asks if an attempt to create an output file should be made. If so, it requests the name of the output file. After completing this task, control is returned to the Main Menu. This can be useful when creating multiple decks in which only a few parameters change.

Choice $=12:$ Store partial input in BLOKxxx file

This option writes the file BLOKxxx where the values for $\mathrm{xxx}$ are determined through input. After completing this task, control is returned to the Main Menu.

Choice $=13:$ Exit program without saving data

Selection of this value exits the program. Control is returned to the operating system. 


\subsection{General Problem Definitions}

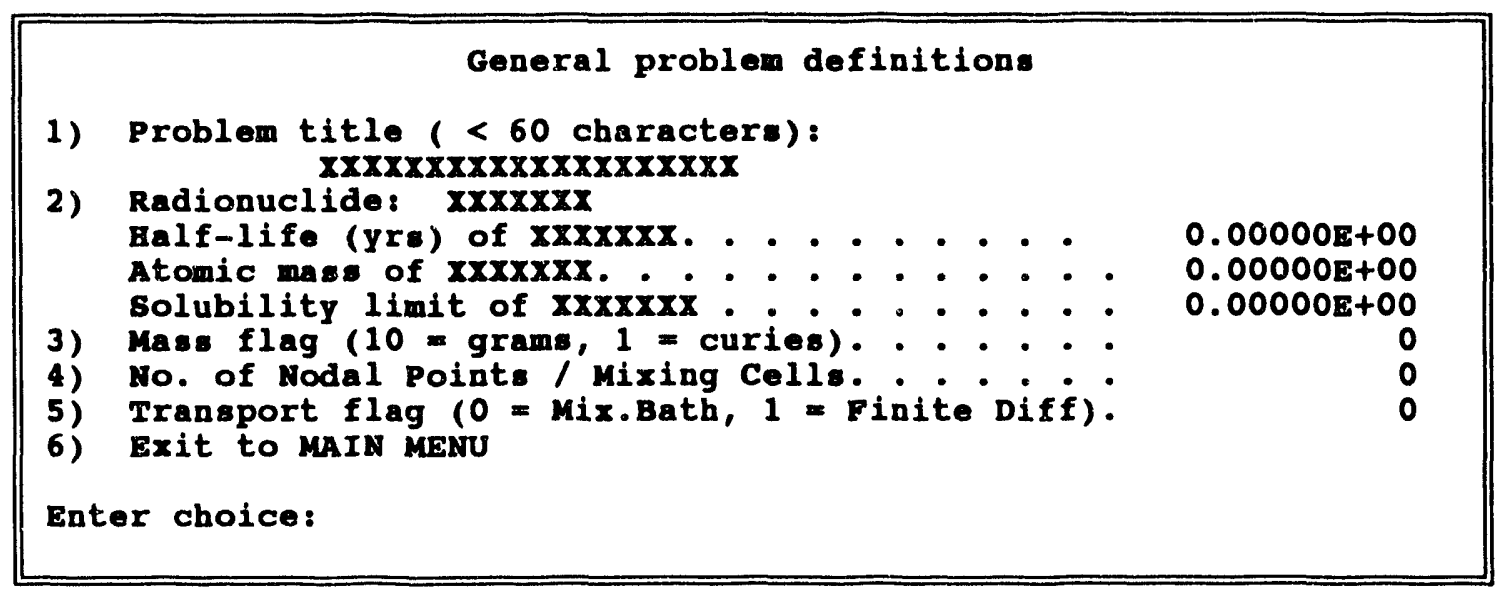

On every sub-menu the most recent values for the various parameters are printed when the menu is accessed. The XXXX values for alpha-numeric characters indicate input has not been specified. In this example, the user is creating a new deck and nothing has been specified. If the user had read in an existing input file, the values would be those found in that file.

\subsubsection{Problem Title}

An alpha-numeric string up to 60 characters in length that describes the problem. Use of the character "/" causes problems when creating BLOKxxx files because the namelist procedure takes this as a variable delimiter. Therefore, use of $" / "$ is not recommended.

\subsubsection{Radionuclide}

DUSTIN has an auxiliary file called RNUCL.DAT that contains over 200 radionuclides, their half-life in years, atomic mass, and a default solubility limit of $10 \mathrm{gm} / \mathrm{cm}^{3}$. The atomic mass is used when converting mass between curies and grams. The solubility limit is used in controlling release. It is well known that solubility values are highly dependent on the environment. The default value has been selected to be large enough that solubility limits will not influence release. If better values for this number are defensible, they can be input to the code. When choice 2 is selected, the following menu is displayed: 


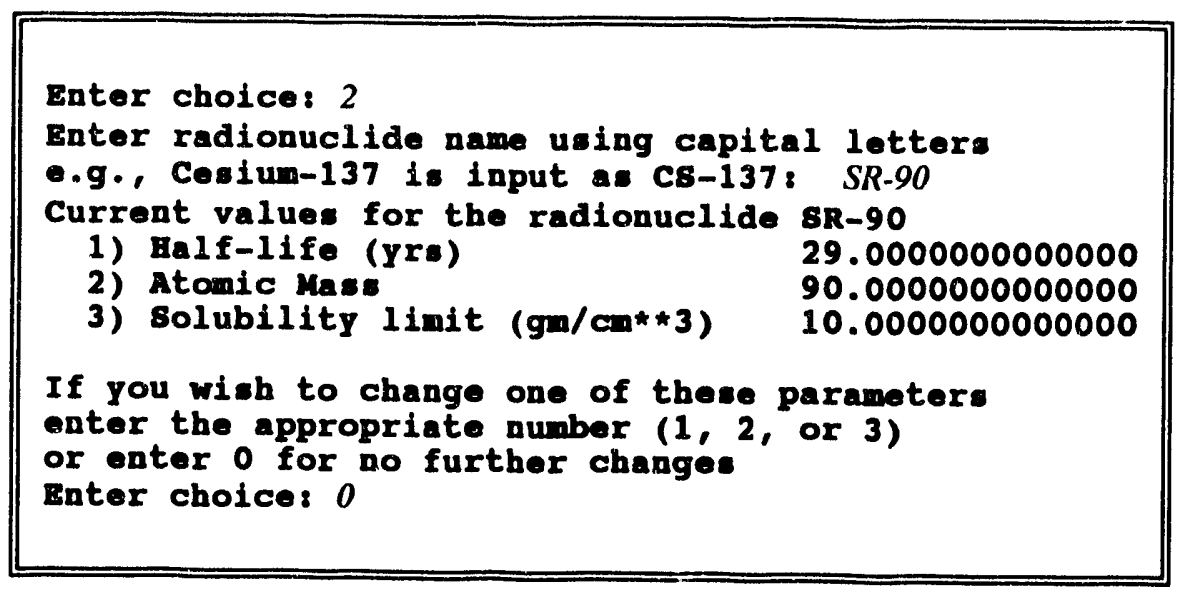

Capital letters are required for the radionuclide name because the computer code takes the input name and performs a string comparison with the radionuclides on the file RNUCL.DAT. If the radionuclide name does not match any of those on file, the code allows the user to either try another name, or enter the values for half-life, atomic mass and solubility limit.

\subsubsection{Units Flag for Mass Input}

Mass can be input in units of curies or grams. If the input is in curies, the code uses the half-life and atomic mass to convert to units of grams. This is done for consistency with $\mathrm{Kd}$ values, wasteform partition coefficients, and solubility limits. For user convenience all output is converted back to the original input units. That is, if the input is in curies, the output is in curies.

\subsubsection{Number of Computational Cells}

The number of nodal points (FD method) or mixing cells (MCMC method) are input. Currently, the maximum value is dimensioned at 500 .

\subsubsection{Transport Flag}

Flag that selects the method of solution for the transport equation. Enter a value of 1 for the finite difference method. The MCMC method is the default. 


\subsection{Time Parameters}

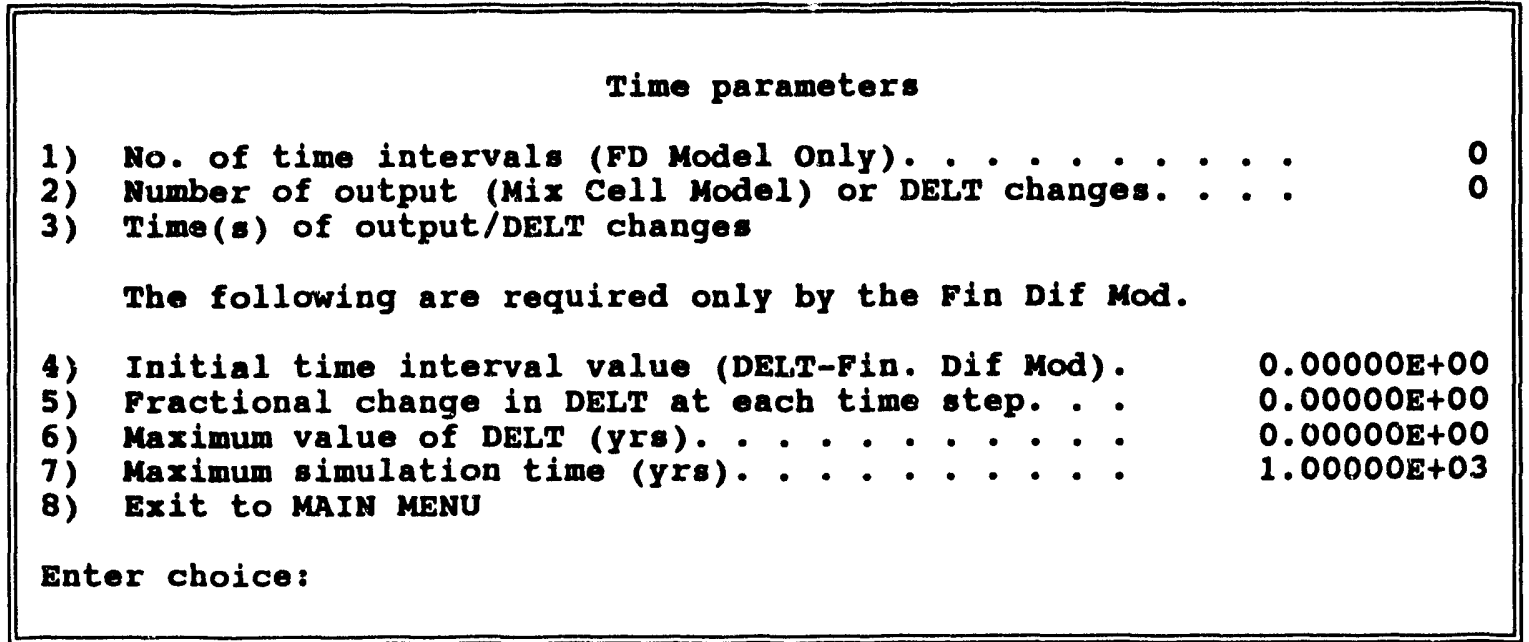

\subsubsection{Time Intervals (FD)}

The maximum number of time steps permitted in solution of the finite difference transport equations. Maximum value is 1000 . Unused in the MCMC model.

\subsubsection{Number of Output (MCMC) or Time Step Changes (FD)}

This input variable, (NDTCHG within the code), serves a dual role depending on the choice of transport model. If the MCMC model is selected, the user must supply the number of times at which a calculation is performed.

If the FD model is selected, the time step size is determined from the initial time step and a multiplier applied at the end of each time step. The size of the time step is limited by a maximum value of the time step, (DELMAX). For example, if the time step is 1 , and the fractional multiplier is 0.1 . The second time step is 1.1 , the third is 1.21 , etc. The increase is applied until the maximum value is reached, Sections 6.2.4 - 6.2.7. Time step logic within the code permits the time step to be reset to the initial time step at user specified times. This can be useful if it is known a priori that an event that will change the movement of contaminants will occur at a specific time. For example, if it is known that containers fail at 20 and 50 years, the user may want to have relatively small time steps around the times of container failure. This can be achieved through resetting the time step at these two times.

\subsubsection{Output Times or Time Step Changes}

In the sub-menu, it should be noted that a value is not listed for this parameter. This occurs because this parameter requires an array of values. Upon selection of this sub-menu item, 
the current values in the array are printed. All parameters that require an array are presented in this fashion.

Again, this is a dual role variable. For the MCMC model this input specifies the times (in years) at which output is requested, with one value for each output time requested in 6.2.2.

For the FD model, this input specifies the time at which the time step is to be reset to its initial value. If this value is greater than the maximum problem time, the time step is never reset.

\subsubsection{Initial Time Step (FD)}

Initial time step size in years.

\subsubsection{Fractional Time Step (FD)}

The fractional change in time step is input in this section. Assuming that the maximum time step has not been reached and the time step has not been reset, the time step size at the N-th calculation is:

$$
\mathrm{DT}_{\mathrm{N}}=\mathrm{DT}_{\mathrm{i}}^{*}(1+\mathrm{FRX})^{(\mathrm{N}-1)}
$$

where $\mathrm{DT}_{\mathrm{N}}=$ the $\mathrm{N}$-th time step.

$\mathrm{DT}_{\mathrm{i}}=$ initial time step, and

FRX = fractional change in time step.

At the time at which the time step is reset to its initial value, the value of $\mathrm{N}$ is reset to 0 .

\subsubsection{Maximum Time Step (FD)}

Maximum value for the time step (years). This value should be selected based on the problem being modeled and the degree of accuracy required in the solution.

Although the solution procedure used is fully implicit, as a rule of thumb, the contaminant should not move more than the width of a computational cell in one time step. Therefore, the following relationship should be maintained:

$$
\frac{V_{D} \Delta t}{R \Delta x}<1.0
$$

where $V_{D}$ is the Darcy Velocity, $\Delta t$ is the time step size, $R$ is the retardation coefficient and $\Delta x$ is the cell width. The above expression can be used to obtain a maximum time step. 


\subsubsection{Maximum Simulation Time (FD)}

Maximum problem time (years), the default value is 1000 years. In the MCMC model, this is automatically set to the last value for the output time.

\subsection{Material Assignments/Properties}

If the MCMC model has been selected, the analytical solution permits only one material type in the facility. Therefore, the DUSTIN code does not permit the user to redefine material types and sends the user to the material property definition menu. The following screen appears in this case.

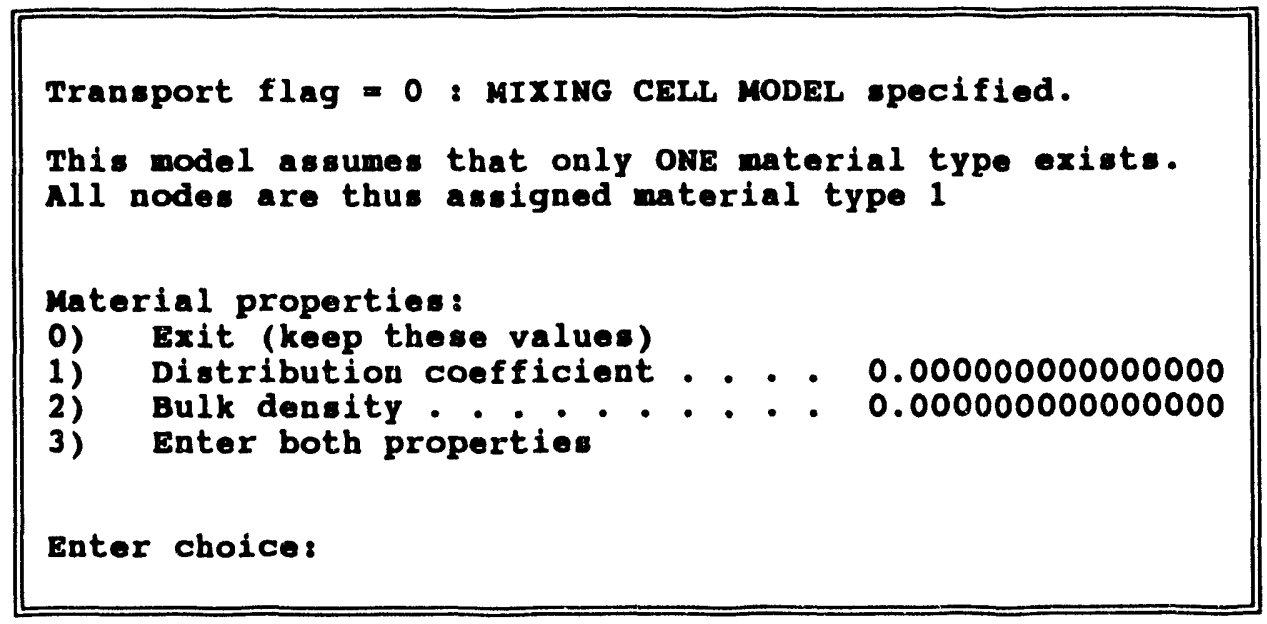

The user can then modify the two material properties used in the MCMC model, the distribution coefficient $\left(\mathrm{cm}^{3} / \mathrm{gm}\right)$ and the soil bulk density $\left(\mathrm{gm} / \mathrm{cm}^{3}\right)$. These values are used to calculate the retardation coefficient.

If the FD transport model is used, up to 10 different material types can be specified throughout the facility. This additional flexibility requires that each control volume is assigned a material type. The code automatically defines all control volumes as material type 1 . Therefore, definition of material type is required only for control volumes that are not material type 1. The FD model material properties menu is:

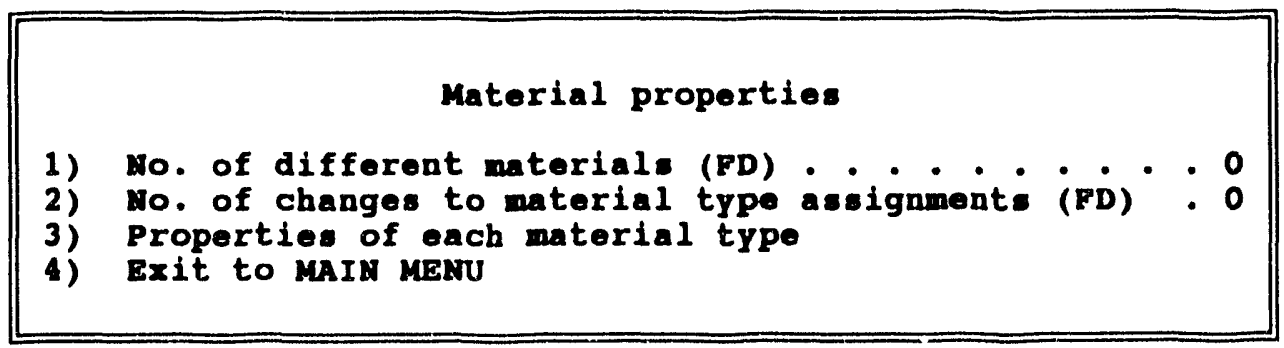




\subsubsection{Number of Different Materials}

The variable specifies the number of different materials in the facility. The maximum value is 10 .

\subsubsection{Material Type Assignments}

Material type reassignment is achieved through the following routine. Explicit instructions and variable definitions are provided when running DUSTIN as in the example that follows.

In this example, the code asks for the number of material type reassignments. To minimize input errors, the DUSTIN code uses a routine that requires the user to input values within a specified range. The maximum and minimum of the range are determined internally by the code and are based on consistincy with previously defined values. In this example, the maximum value of 100 is determined as the maximum number of control volumes as specified in Menu 1.4.

In this case, the code user asked to reassign 10 nodes (control volumes). The code then prints the instructions and definitions for the input variables. The first of which is the node number for the first node in this sequence. Again, Min and Max values are calculated by the code. The user selected a value of 90 .

The code asks for the number of nodes to be reassigned in this sequence. The user requested 10 , the total number previously specified. If the first node in the sequence was 95 , the code would permit only 6 nodes (nodes 95 - 100 inclusive) to be redefined. For the increment between nodes, the code calculated that the maximum increment was 1 and forces the code user to input a value of 1 . If for example, the user asked to redefine 2 nodes starting at node 90 , the maximum increment would be 10 (i.e., nodes 90 and 100 could be defined on this card) and any value between 1 and 10 would be acceptable.

The code asks for the material type to assign to the first node in this sequence. The maximum value of 3 was determined from the value specified in Menu 3.1. The code user selected material type 3 .

The increment in material type to each node was determined to be zero (there are only 3 materials). In a 1-D simulation most often, the materials will occur in layers covering several nodes and zero will be the appropriate choice. However, if the material types change in a regular fashion, this feature may be used.

Upon completing this sequence of input, the code prints out the values and asks if they are acceptable. In the example, we have defined nodes 90 - 99 to be material type 3 . Note, node number 100 is still material type 1. 
Ho. of material corrections (MIMm0,MAX=100):

(Number of nodes whose material type aseignoents

will NOT have the default value of 1)

Enter cholce: 10

Material type re-assignment is achieved through a

sequence of five integer numbers. These numbers ares

1) The location (node () of the firet node in the neq.

2) The number of nodes to be reaseigned.

3) The increment of the nodes in this equence.

4) The naterial type for the firet node in the seq.

5) The increment of material type for the noder in the eeq.

For example, to assign even number nodes between $10-24$

to have material type 2 use the following values:

$\begin{array}{ll}1)=108 & 2)=(24-10) / 2+1=88\end{array}$

$\begin{array}{lll}3)=28 & 4)=28 & \text { 5) }=0\end{array}$

Hode of the firat node of sequence 1

to be re-assignnd: (MIN=1, MAX $=100)$

Enter choice: 90

Ho. of nodes that will be re-aseigned in this

equence: (MIN $=1, \operatorname{MAX}=10$ )

Enter choice: 10

Increment of nodes that will be re-assigned:

(MIN = $1 \operatorname{MAX}=1$ )

Enter choice: 1

Material type of first node in sequence 1:

(MIX $=1, \operatorname{MAX}=3$ )

Enter choice: 3

Increment of the material type index for the subsequent nodes in the sequences (Normally this would be zero, 1.0 , all nodes in the sequence belong to the same material type. It is non-zero if they change in a linear manner.)

(MIA $=0, \quad$ MAX $=0$ )

Enter choice: 0

Values for this card (sequence) are:

$\begin{array}{ccccc}\text { First } & \text { Number } & \text { Increment } & \text { Material } & \text { Increment } \\ \text { Node } & \text { in seq } & \text { Node } & \text { Type } & \text { Mat'l Type } \\ 90 & 10 & 1 & 3 & 0\end{array}$

0 - Re-enter TaIs sequence

1 = Continue

Enter choice: 1

Material type re-assignment complete.

$\begin{array}{ccccc}\text { First } & \text { Number } & \text { Increment } & \text { Material } & \text { Increment } \\ \text { Node } & \text { in seq } & \text { Mode } & \text { Type } & \text { Mat' } 1 \text { Type } \\ 90 & 10 & 1 & 3 & 0\end{array}$


The code checks to determine if the number of material type reassignments equals the total number specified in Menu 3.1, if it does, the sequence of numbers that define material type are printed. If it does not, the code returns to the beginning of this section and asks for more reassignments. Although there is a check on the total number of reassignments, there is no check on whether a node has been reassigned twice. For example, the user could specify that nodes 1 - 5 are material type 2 in the first sequence and then specify nodes $3-7$ are material type 3 in the second sequence. The code would then only define 7 nodes having material properties different than material 1.

\subsubsection{Material Properties}

For the FD model, four soil material properties (distribution coefficient, bulk density, dispersivity, and diffusion coefficient) are required. The following example provides a typical input session for the first material. First, the code prints out the existing values for all material types (specified in Menu 3.1). If the user decides to change some of these properties, the code asks for the material type to change, prints out the existing values for that material and allows the user to change any one of the values independently or all of the values simultaneously. In this example, all of the values are changed. The units required for the various parameters are printed to remind the user that cgs units are used for material properties. After the user has input the parameters, the code prints these values and allows the user another chance to change the input. If the input is correct, the code returns to the materials properties menu and asks for changes in other materials. 


\section{Material properties:}

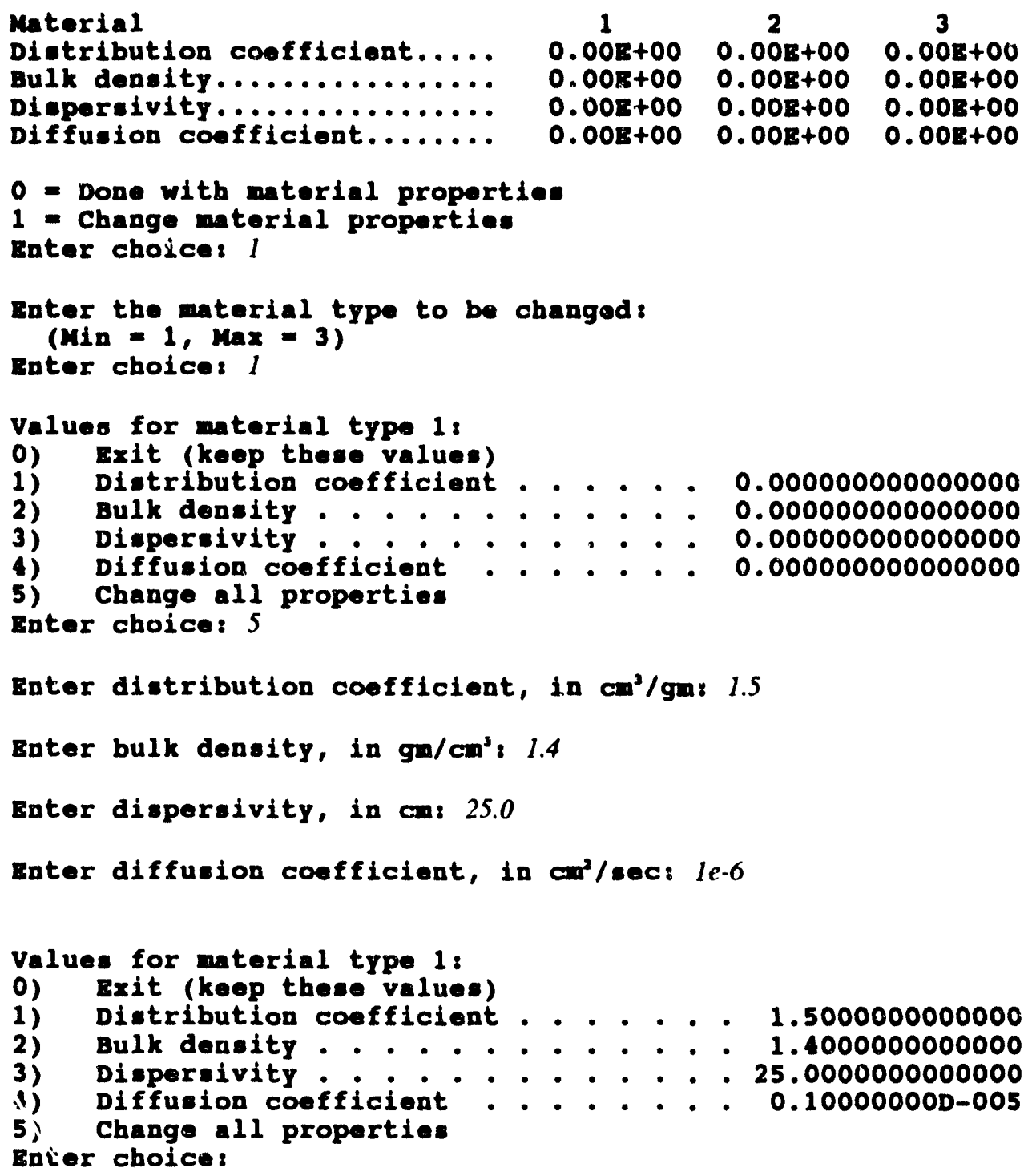


This sub-menu controls output produced by the DUST code.

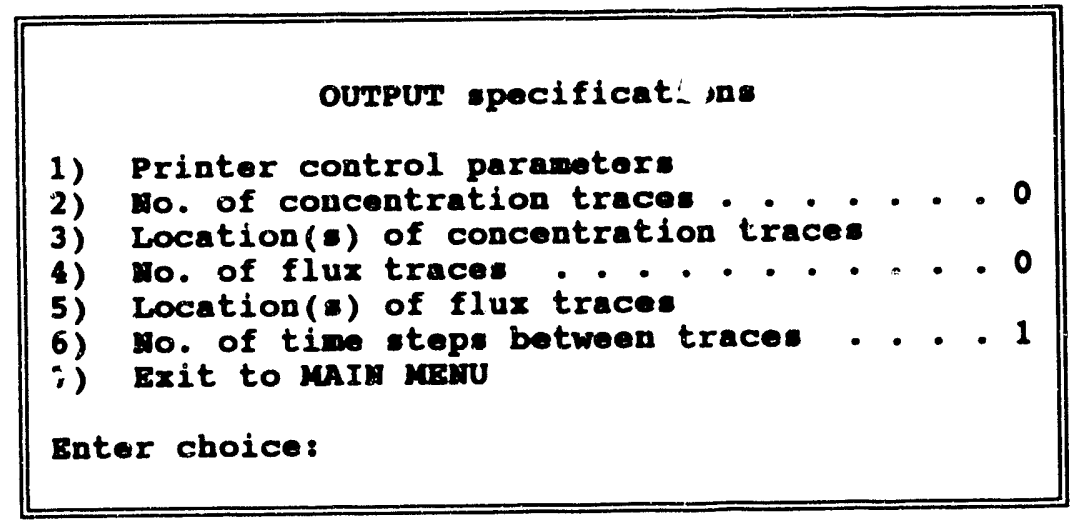

\subsubsection{Printer Control Parameters}

The first menu item controls the main print out. In DUST ther is a variable KPR(I) where I ranges from 1 to the number of time steps in the FD model and to the number of output times in the MCMC model.

For the FD model, if $\operatorname{KPR}(I)=4$, nothing is printed at the I-th time step. If $\operatorname{KPR}(I)=1$, the concentration at each finite difference point is printed. If $K P R(I)=2$, the concentration and flux are printed. If $\operatorname{KPR}(I)=3$, container breach and wasteform release parameters are printed. When using the FD model, often many intermediate calculations are performed to enhance numerical accuracy, therefore, zero is the default value for all items in KPR. Thus, in order to ottain the main output, some of the values for KPR must be specified. At every time step in which KPR $>0$, the concentrations at every location are written to the file CONCNT.DAT. This file can be used by the program GRAFXT to graph the data on the video console.

For the MCMC model, output is slightly different. In the MCMC model predictions are obtained from an analytical solution which is calculated only at the times and locations specified through input. The times for output are specified in Menu 2.3 and the locations are specified through the trace files, Menu 4.3 below. Again $K P R(I)=0$ prints nothing. However, KPR(I) $=1$ prints the concentration and fluxes at the trace locations specified. The MCMC model does not have any temporal output pertaining to release from the wasteform or container breach. In this model, container breach is specified via input as the time to failure and wasteform releases are either exponentially decaying in time, in which case, the amount released can be easily determined, or release is instantaneous due to surface rinse. The default value for $\mathrm{KPR}(\mathrm{I})$ is one since calculations are only performed at requested times. 


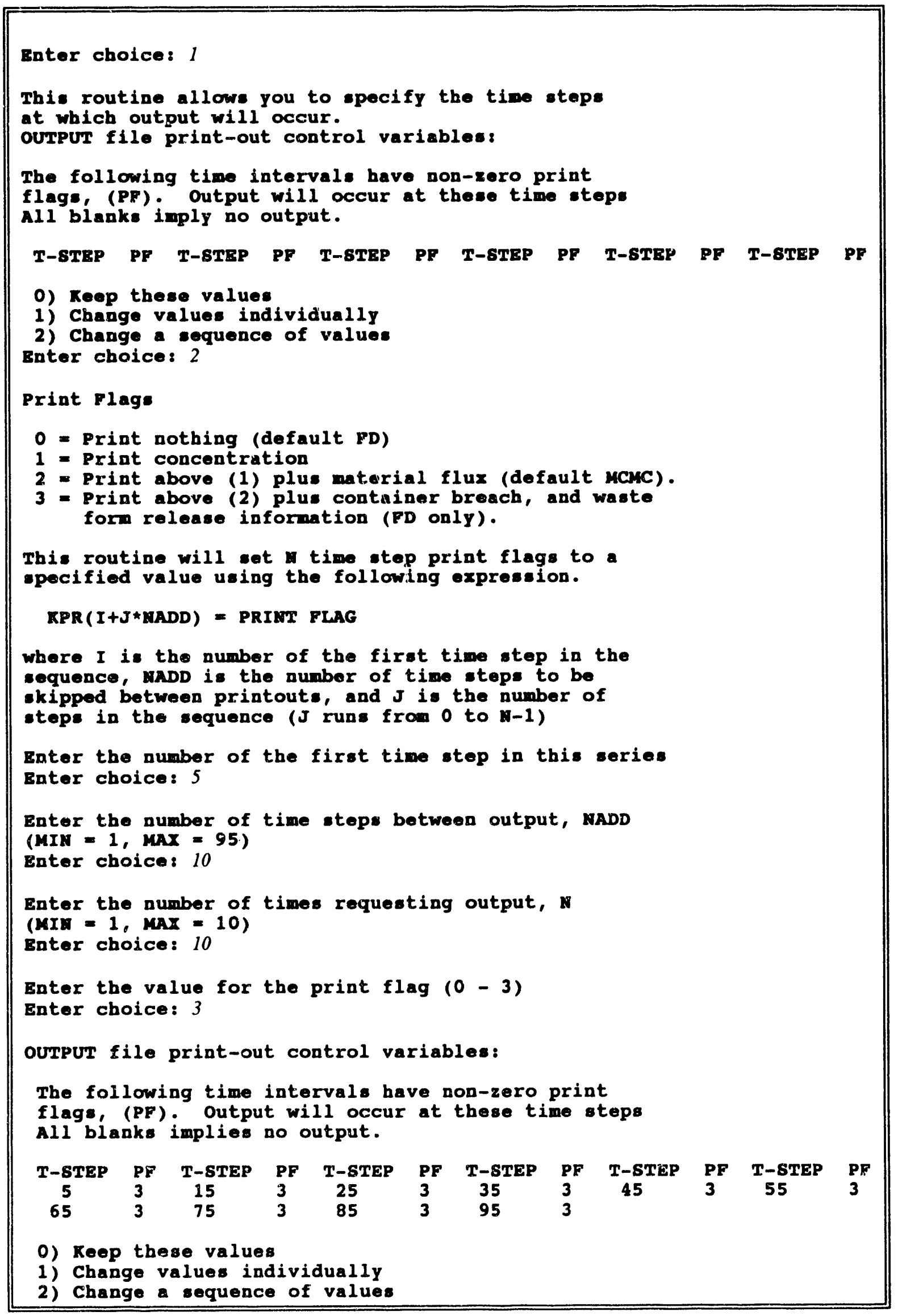


In the preceding example, the FD model has been specified and initial values for KPR are zero. The user selects to obtain the full printout, $K P R=3$, starting at time step 5 and for every 10-th time step after this. Instructions on how to input this information are provided while running the code as shown. After specification is complete, the code returns to the output specifications menu.

If option 1, change values individually, were selected the code asks for the number of values that are to be changed and then uses this number to repeat a sequence of questions asking for the time step and print flag to be specified. Again, instructions are provided during operation of DUSTIN.

\subsubsection{Number of Concentration Traces}

Requesting a concentration trace requires the DUST computer code to write a file containing concentrations at a specified location as a function of time. Up to twenty locations can be specified. The output file created by DUST is called TRACECND.DAT. This file can be used directly by the graphics program GRAFXT.EXE and a plot of concentration versus time can be viewed immediately after running the DUST code.

In the MCMC model the number of concentration and flux traces as well as their locations must be identical. The reason for this is calculations are performed only at the trace locations. The DUST code performs a check to force identical flux and concentration trace locations when the MCMC model is used.

\subsubsection{Location of Concentration Traces}

The location of the concentration traces are determined by specifying the node (or mixing cell) at which a trace should be made. In DUSTIN the user is given the option of redefining a single trace node, or all of the trace nodes. In the following example, trace nodes had been previously specified and the user decided to change the last trace node from 50 to 60 . After this, the code would print out the new values for trace nodes and ask if any further changes were desired. 


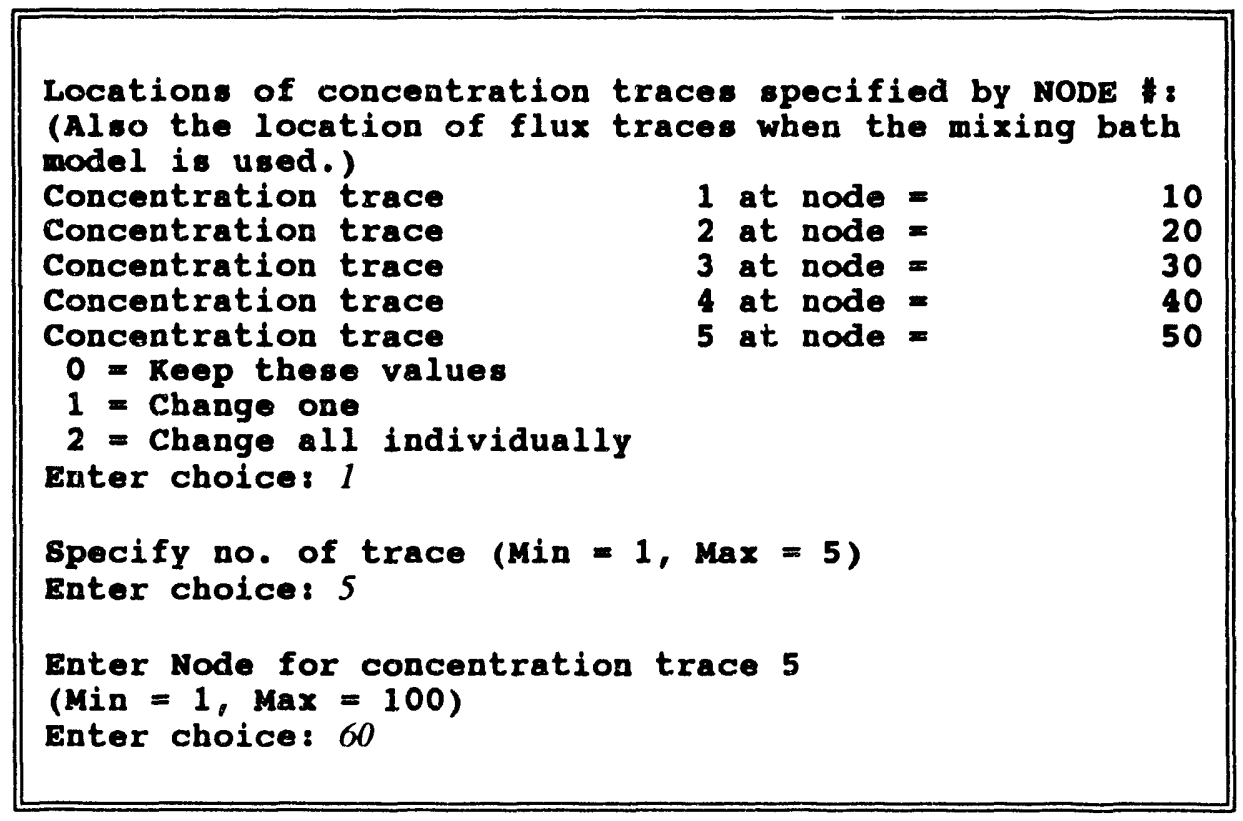

\subsubsection{Number of Flux Traces}

Requesting a flux trace requires DUST to create a file containing the instantaneous mass flux, total mass (flux integrated over time multiplied by the surface area of the facility (Menu 5.2)) that has passed through that point, and mass release rate (flux multiplied by the surface area). The total mass is calculated numerically by summing the product of the flux, facility area, and time step. This estimate may be subject to large errors in the MCMC method if the time between computations is large.

If the MCMC model is specified, DUSTIN does not allow the user to input this variable and refers the user to the concentration trace section. The number of flux traces is automatically set equal to the number of concentration traces.

\subsubsection{Location of Flux Traces}

The location of flux traces are determined by specifying the node (or mixing cell) at which a trace is requested. If the MCMC model is used, DUST requires that the locations of the flux and concentration traces be identical. Therefore, DUSTIN refers the user to the section on specifying concentration trace locations (Menu 4.3). The input procedure for specifying location for flux traces is identical to that of concentration traces.

\subsubsection{Number of Time Steps between Traces}

This value specifies how frequently the trace files are updated. If the default value of 1 is used, traces are written at every time step. If a value of $\mathrm{N}$ is used, traces are written every $\mathrm{N}$-th time step. 


\subsection{Facility Dimensions and Coordinates}

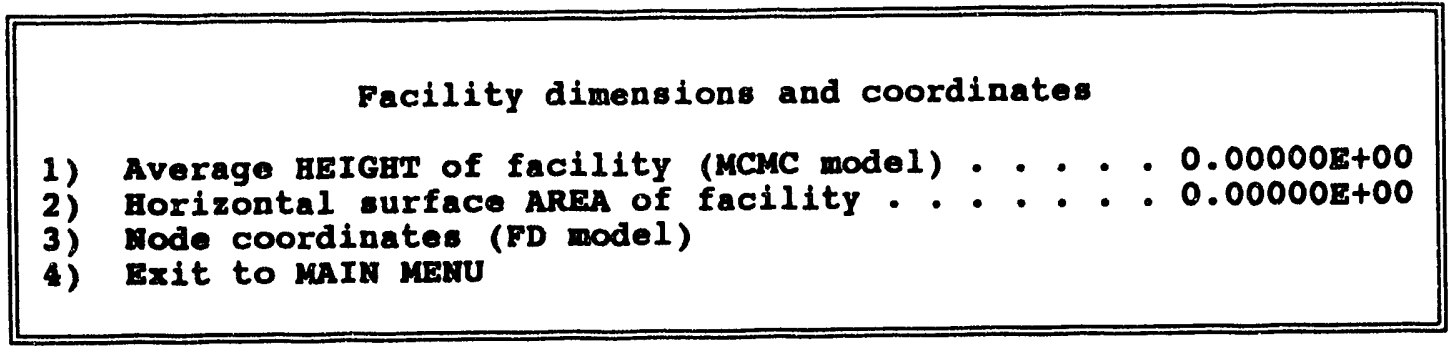

\subsubsection{Height of the Facility}

The MCMC model requires the total height of the facility. The size of each mixing cell is the total height divided by the number of mixing cells. The height must be input in centimeters.

The FD model calculates the facility height directly from the nodal coordinate input and this input is not required.

\subsubsection{Surface Area}

The surface area is a normalization factor that permits scaling of the concentrations (mass per unit volume) to account for the 3-D facility in a 1-D model. The volume of a compu-tational cell is the height of the cell multiplied by the surface area. Obtaining the correct concentration is important as the dose to man scales linearly with concentration. The area must be input in units of square centimeters.

\subsubsection{Node Coordinates (FD model)}

The FD model permits non-uniform mesh spacing. This is useful when modeling different regions which exhibit different transport characteristics. The values for the node coordinates represent depth and can be positive or negative. However, Node 1 is always the highest node (least deep) and the last node is the lowest node (greatest depth). When entering coordinate values, the coordinates must increase with depth and the user must input the coordinates in order, beginning with Node 1 . (The DUSTIN code forces the user to begin with Node 1 and continue sequentially to the last node.) The distance must be in units of $\mathrm{cm}$. For regularly spaced nodes, automatic generation of the nodes can be specified through the formula in the following example.

Upon entering this menu, the following description of the input requirements is presented. 


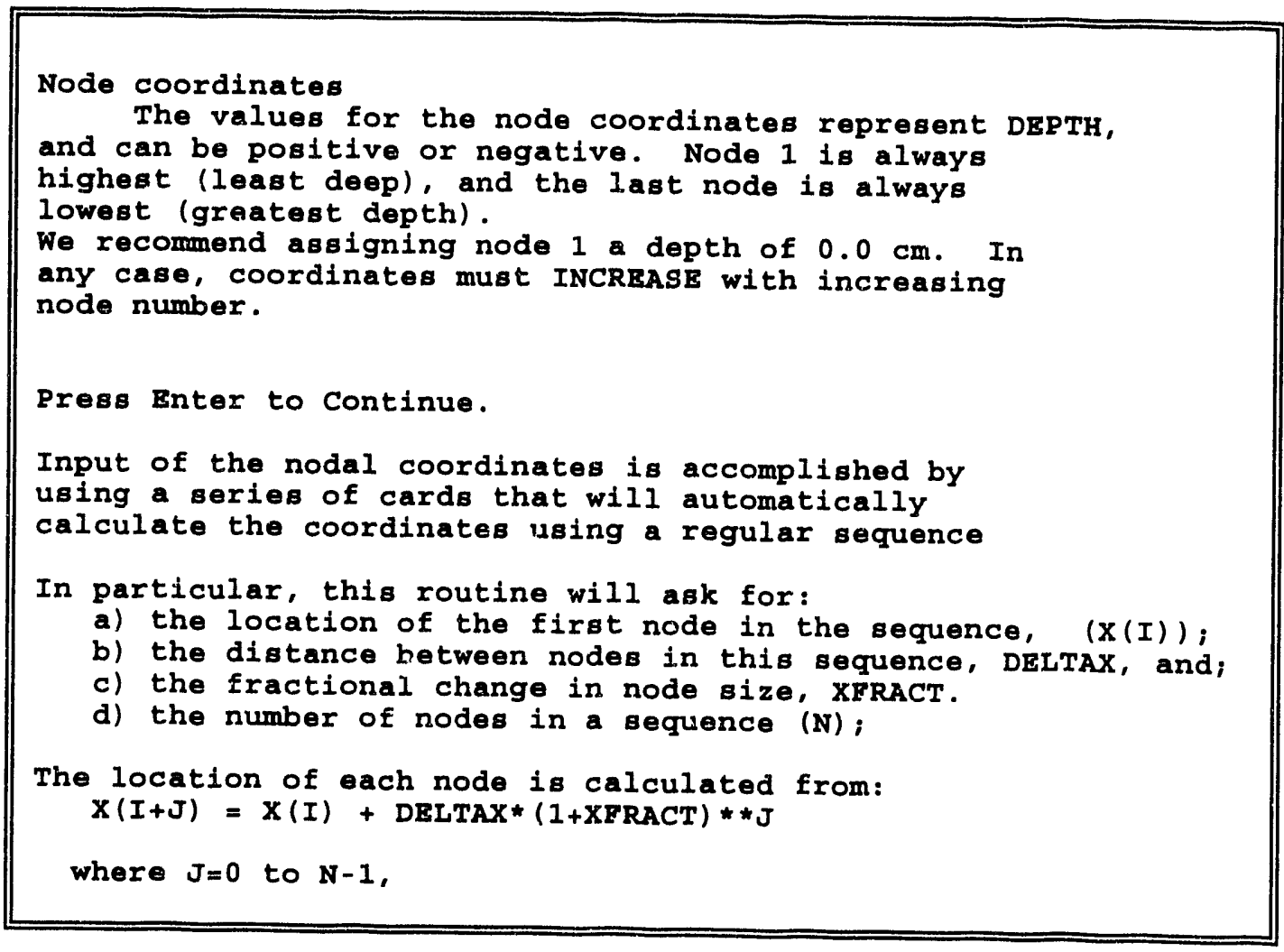

The example below defines nodes $1-50$ having a mesh spacing of $10 \mathrm{~cm}$ beginning with node 1 at $x=0$.

Coordinate of node 1 (cm): 0.0
Distance between each node for the nodes in this
first sequence (cm): 10.0
Fractional change of the distance between nodes over
the preceding distance: 0.0
No. of nodes that will be assigned coordinates in
this first sequence, including node 1
(MIN = 1 , MAX $=$ NNe $=100)$
Enter choice: 50
$0=$ Re-enter this sequence
$1=$ Continue
Enter choice: $l$


The DUSTIN code permits the user to re-enter the sequence if an error occurred. If the sequence is correct, the code automatically checks to make sure that every node receives a coordinate value. In this case, more nodes need to be defined and the code calculates the value of the last node and the number of nodes remaining to be defined, and asks for more input. In this example, nodes $51-100$ have a mesh spacing of $20 \mathrm{~cm}$ beginning with $X(51)=500 \mathrm{~cm}$.

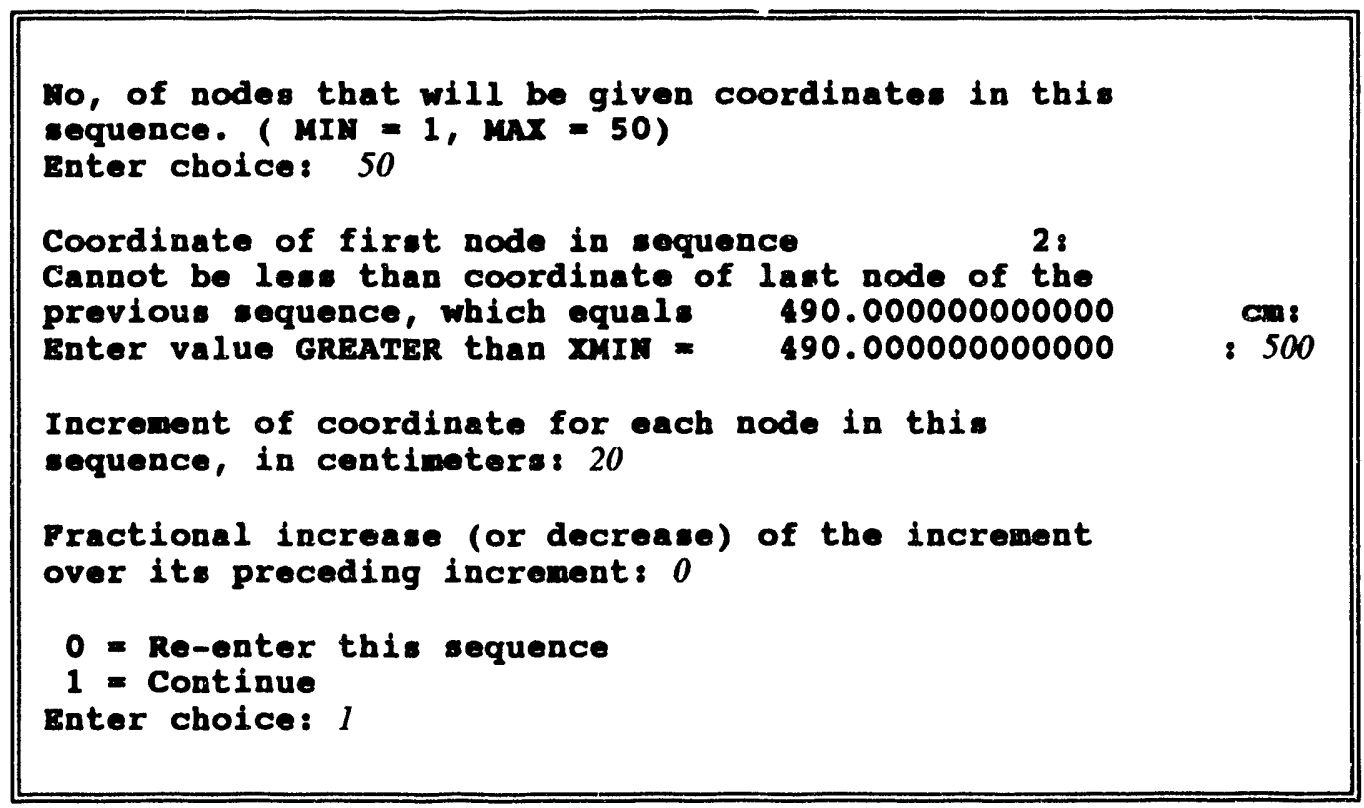

After completion of the input, the code prints the values requested in the form used by the DUST code.

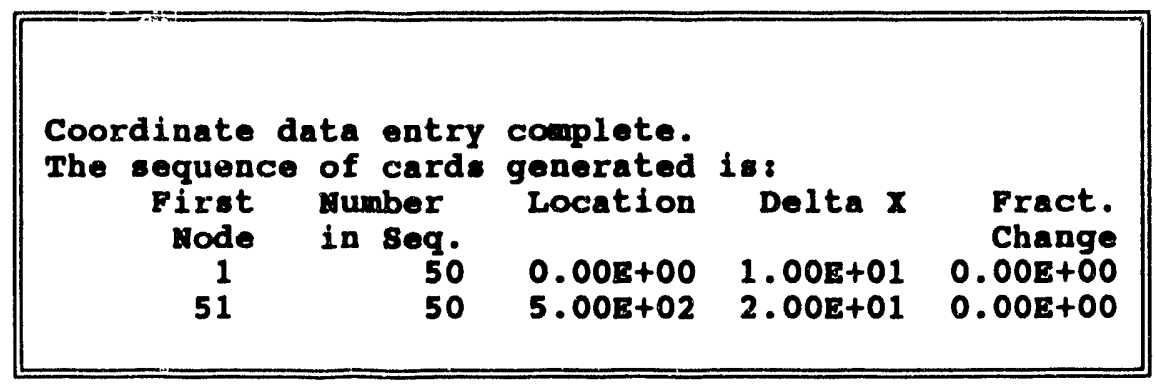

\subsection{Initial and Boundary Conditions}

The MCMC model assumes that the concentration entering the top of the modeled region is zero and because of the uni-directionality of flow, does not require a bottom boundary condition. Therefore, only the initial conditions are required. If the initial condition is zero concentration everywhere, this is the default and the code will automatically generate this when requested. If there are non-zero initial concentrations, the user specifies these through three input 
parameters, the number of locations to be given a value, the value, and the amount to add to that value when incrementing the location counter by 1 . This procedure will be explained in detail under the FD model initial conditions section.

The following is a typical example of the Boundary and Initial Conditions session, if the transport flag indicates that the MCMC model is to be used.

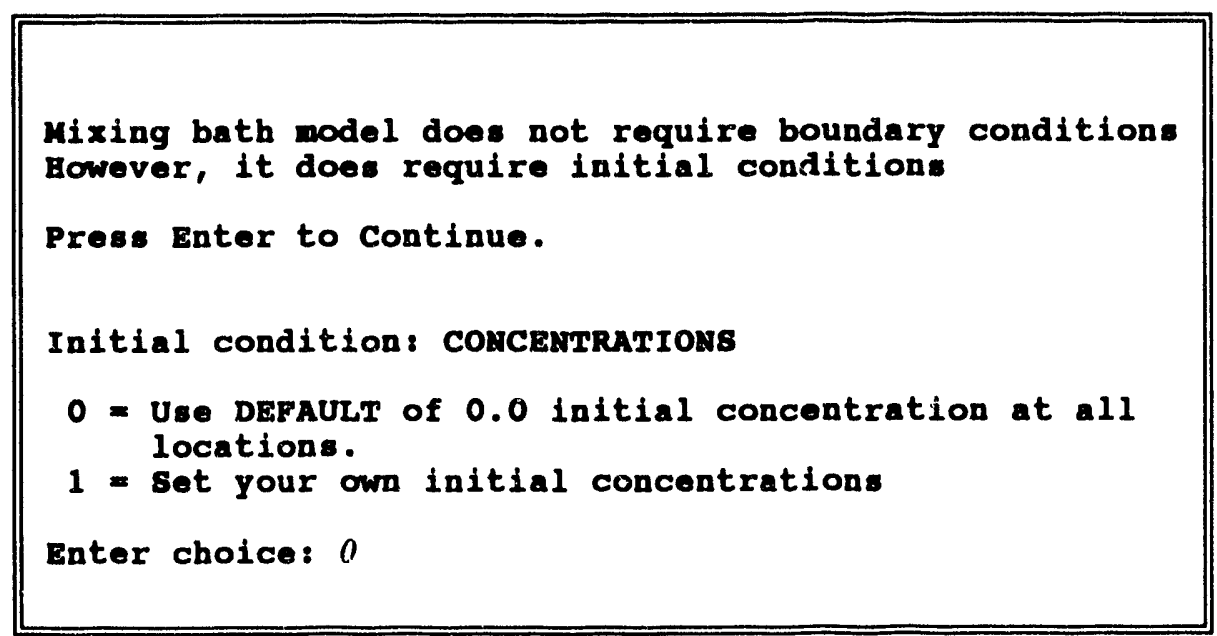

If the FD transport model is being used, the following menu appears.

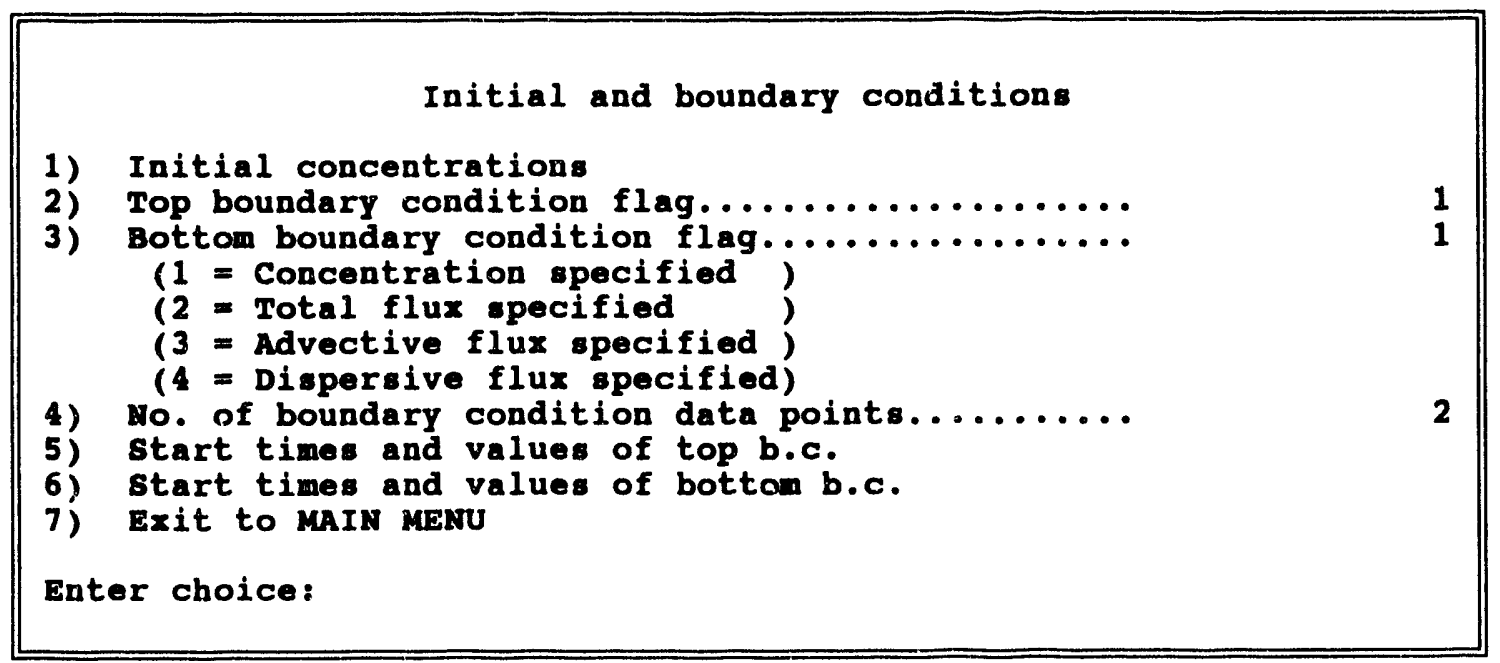

\subsubsection{Initial Conditions}

The initial concentration at every finite difference point must be specified. If a number of concentrations are equal or change in a regular manner, the input can be greatly simplified by 
entering a sequence of three numbers which represent the number of nodes (locations) at which the concentration is to be defined, the value for the concentration at the first of these nodes, and the amount to add to each successive node. DUSTIN requires that the initial conditions be specified beginning with Node 1 continuing sequentially to the last nodal point. Upon completion of specifying all concentrations, the sequence of values are printed and control is returned to the Boundary and Initial Conditions submenu. If the initial condition is zero everywhere, the user can request the code to generate the appropriate input automatically.

The instructions printed by DUSTIN when entering this menu are:

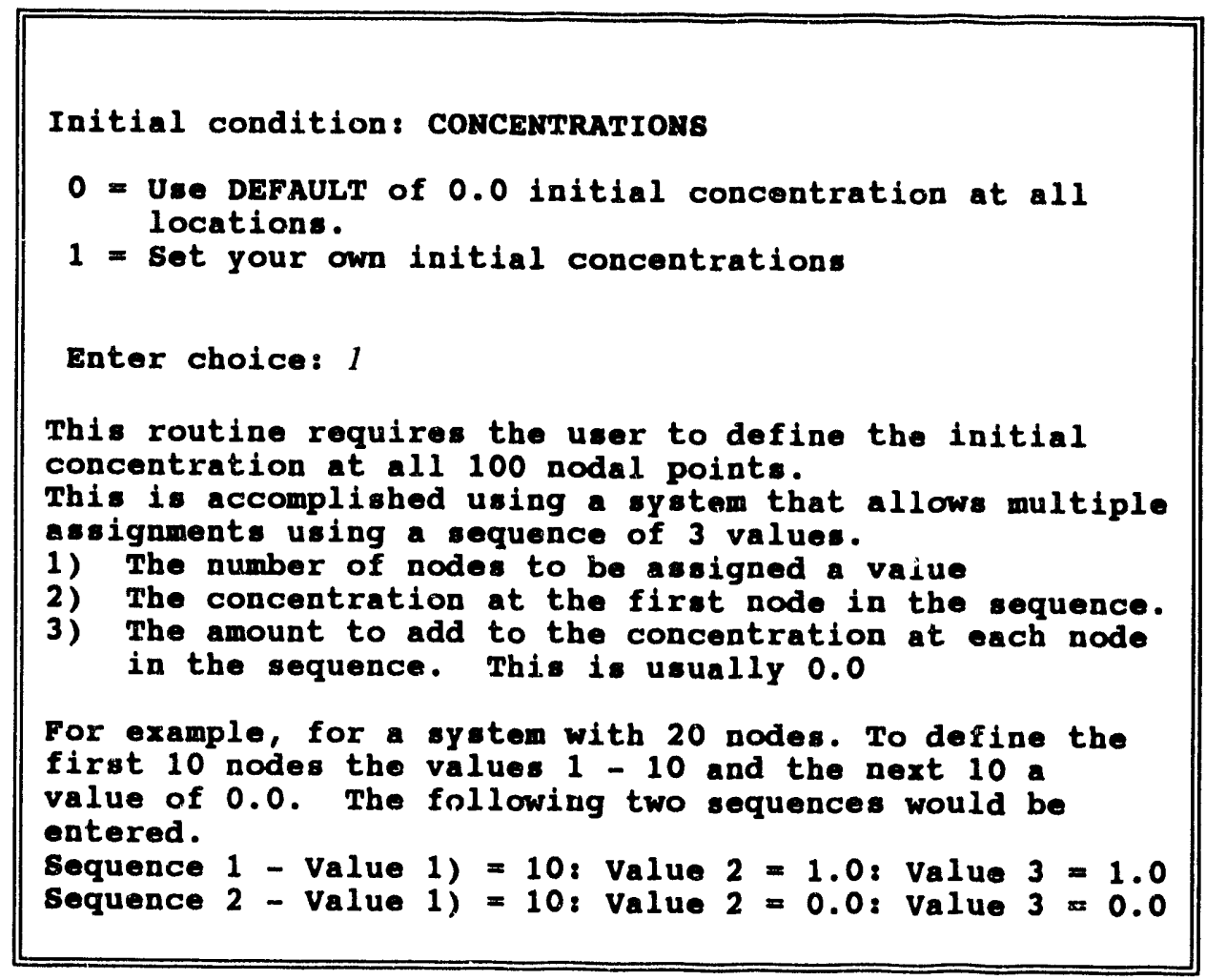

An example in which 100 nodal points have been requested follows. The first 50 nodes have an initial concentration of one. The units of concentration are either $\mathrm{gms} / \mathrm{cm}^{3}$ or $\mathrm{Ci} / \mathrm{cm}^{3}$ depending on whether the mass inventory is input in units of grams or curies (Menu 1.3 Mass Flag). 


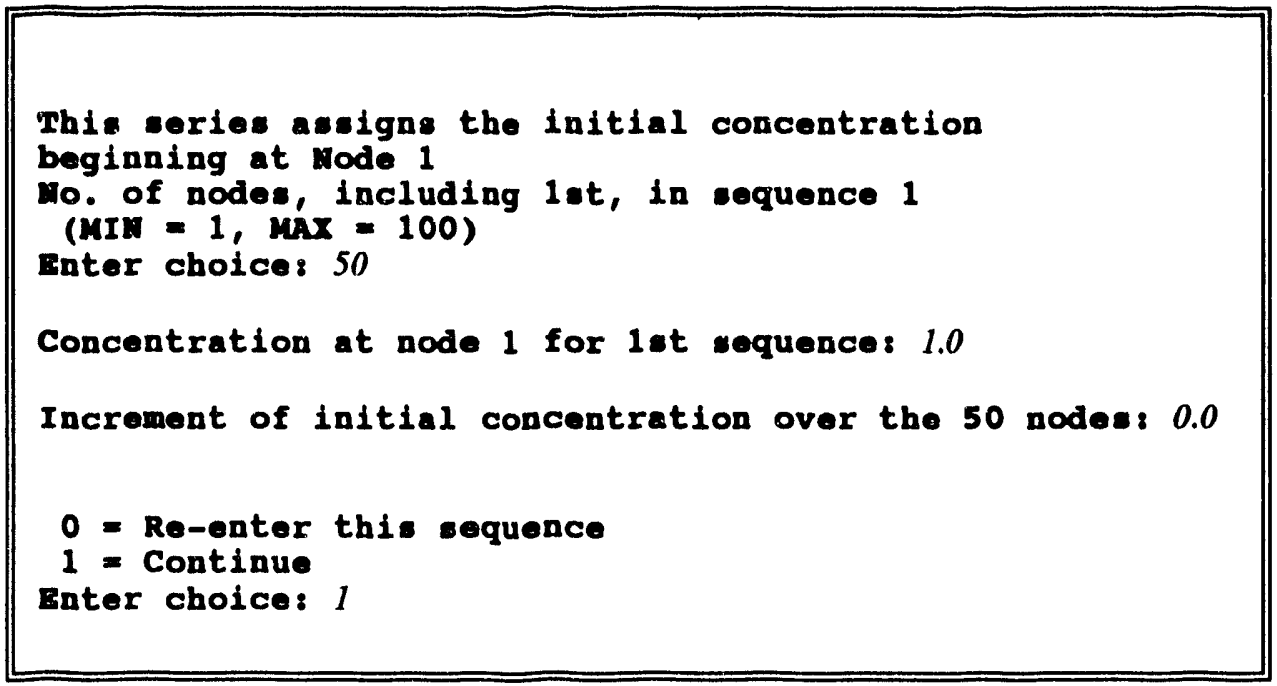

At this point, the code recognizes that not all 100 nodes have been given an initial concentration and more data is requested.

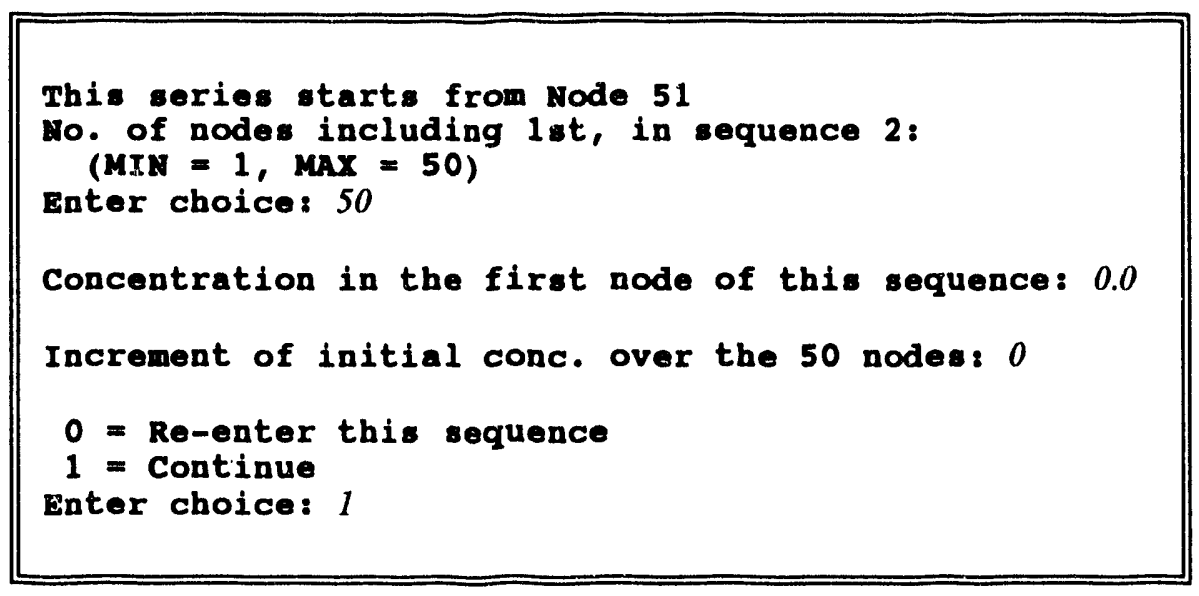

At this point, all nodal points have a specified initial condition and the code prints out the input values in the form required by the DUST code.

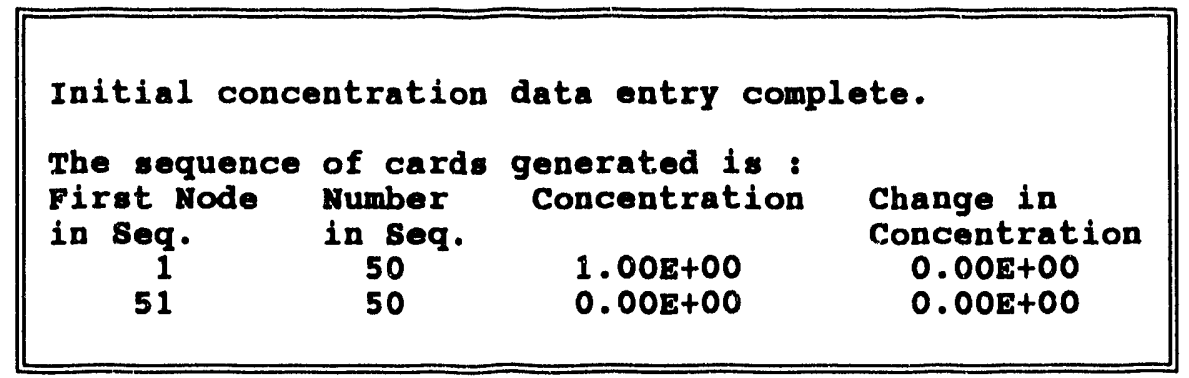




\subsubsection{Top Boundary Condition Flag}

Four choices for boundary conditions are allowed within the DUST code. The concentration, total flux, advective flux, or diffusive/dispersive flux can all be specified as a function of time. These are described in detail in Section 2.6.

This input value selects the boundary condition from among these choices. The default value requires the concentration to be specified.

\subsubsection{Bottom Boundary Condition Flag}

The choices are identical to those at the top boundary as described in 6.6.2.

\subsubsection{Number of Boundary Condition Data Points}

All boundary conditions are specified as a function of time through a table containing the value of the boundary condition and the time in years. At any given simulation time, the value of the boundary condition is determined through interpolation of this table. The last time in the boundary condition table should be greater than or equal to the maximum simulation time. If this is not done, unpredictable results may occur. The minimum number of data points required to specify the interpolation table is 2 . The maximum number allowed by the code is 10 .

\subsubsection{Boundary Condition Table for the Top Boundary}

Boundary conditions (BC) are input as ordered pairs of time (years) and $\mathrm{BC}$ value (where the units are consistent with previous input, e.g., if mass is input in curies, mass units for the BC are in curies).

The following example has 4 data points in the table (specified in Menu 6.4), a maximum simulation time of 100 years (specified in Menu 2.7) and sets the concentration to 1.0 between 0 and 10 years and 0.0 after 10.01 years. Between 10 and 10.01 years the concentration varies linearly between 1 and 0 . Upon entering this submenu, the code prints out the existing values for the $\mathrm{BC}$ (all zero by default in the example). After a decision has been made to change the values, the code prompts the user for the first concentration value and forces the first time in the table to 0.0 years. After this, the code prompts the user for the time and boundary value. These

are input on a single line separated by a space. After all of the boundary points are specified, the table is printed for review by the user. 


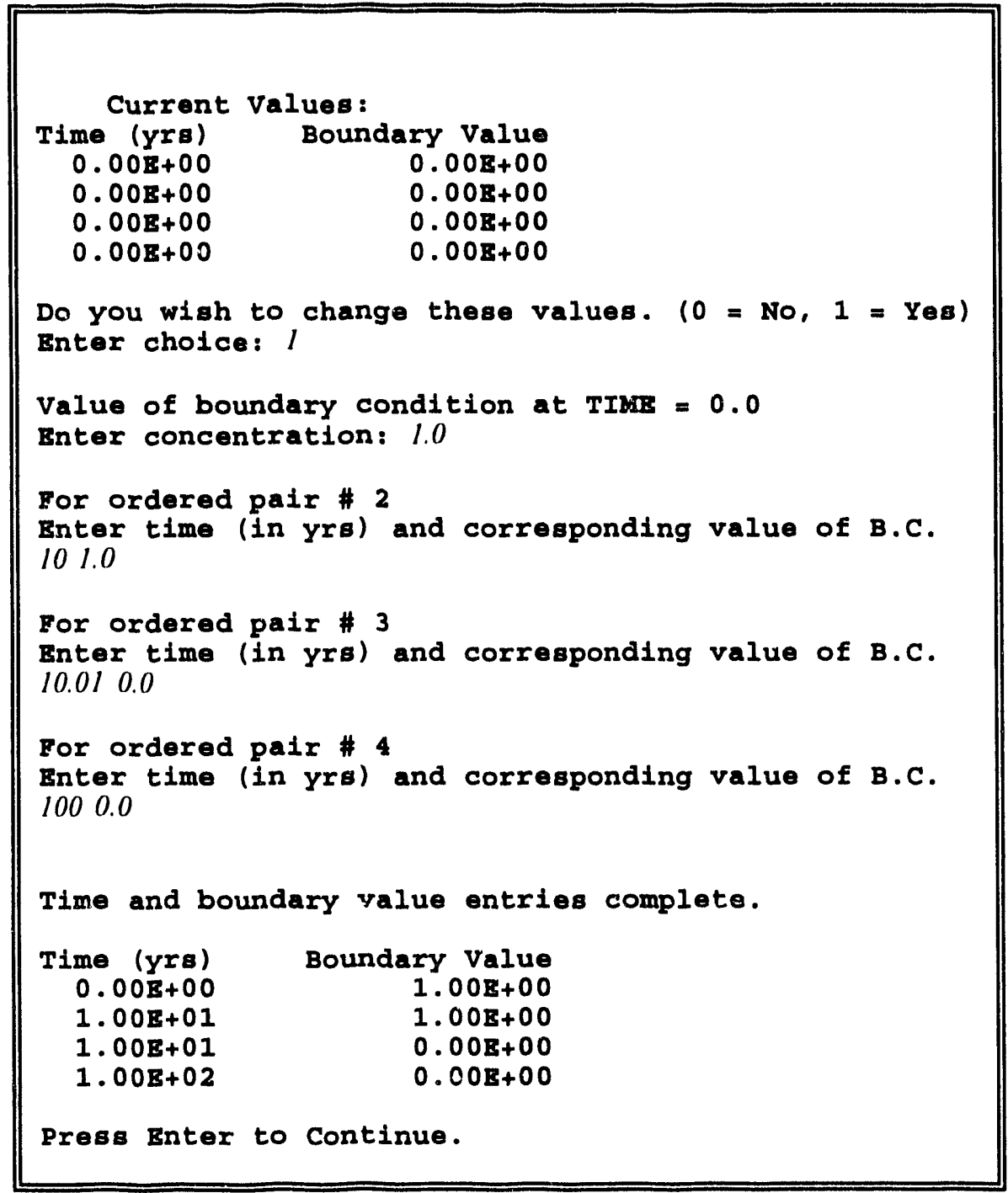

6.6.6 Boundary Condition Table for the Bottom Boundary

This procedure is identical to that of the top boundary. For details see Section 6.6.5.

\subsection{Water Flow and Moisture Content}

For the MCMC model, the user is permitted to input a single value for the Darcy velocity and the moisture content. The FD model permits the Darcy velocity to be obtained as a function of time through tabular input in an approach similar to that of specifying the boundary conditions. The moisture content is permitted to vary spatially. The values for the Darcy velocity and moisture content should be obtained from numerical simulations with site specific data. The menu is: 


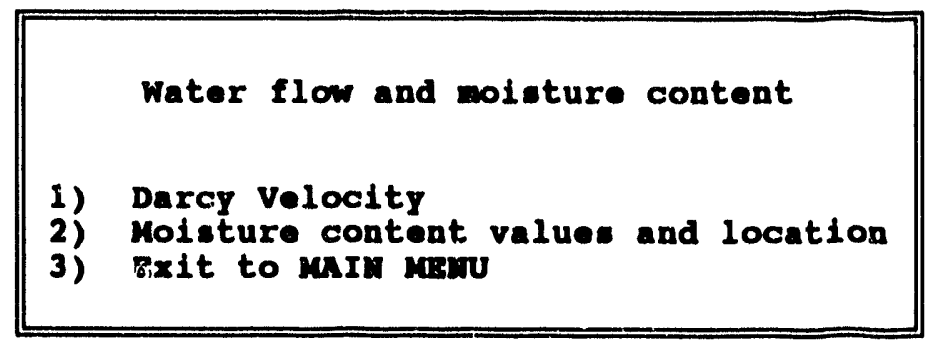

\subsubsection{Darcy Velocity}

For the MCMC model, the DUSTIN code prints the current value for the Darcy velocity, asks if a change is needed, and if so, asks for the value of the Darcy velocity $(\mathrm{cm} / \mathrm{s})$.

For the FD model, a table of velocity versus time may be input or a single value for the Darcy velocity may be used. If a single value is used, the code asks the user for that value and creates the input table by specifying this value at $t=0$ and $t=$ TMAX (Menu 2.7). If a table is input, the velocity at any simulation time is calculated through linear interpolation using the table. If the last time value in the table is less than the maximum simulation time, unpredictable results may occur.

In the following example, the user has decided to specify an increase in velocity over time between 0 and 1000 years. DUSTIN prints current values for the table ( 0 by default) and asks if a change is requested.

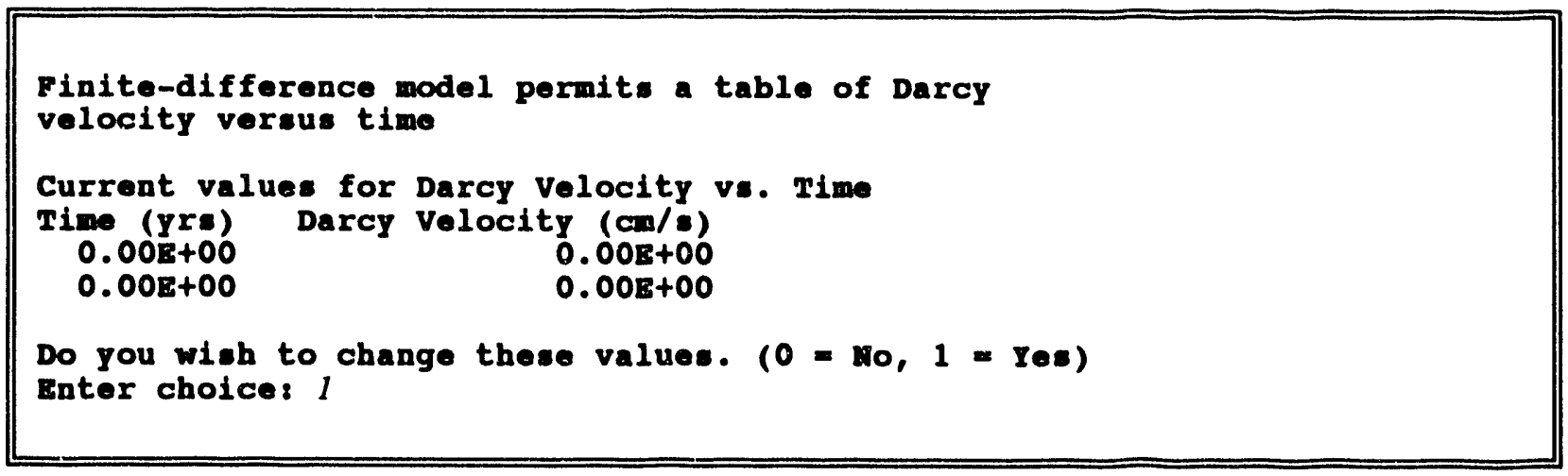

At this point, the user is provided a choice of using a single value or inputting a table of values. If a table of values is desired, the user inputs the number of points in the table and the code then prompts the user for the values. The code forces the first time value to be at zero years. 


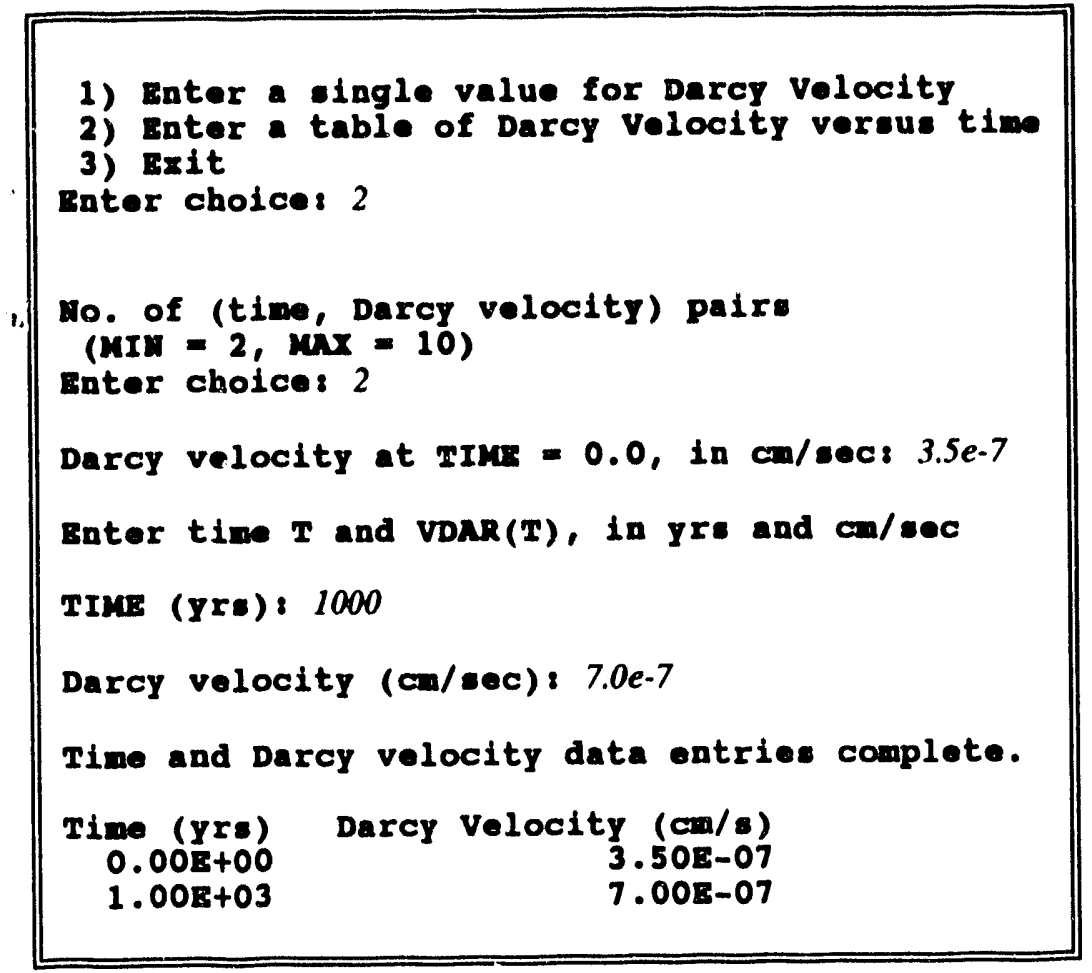

\subsubsection{Moisture Content Values}

For the MCMC model, the DUSTIN code prints the current value for the moisture content, asks if a change is needed, and if so, asks for the value of the moisture content. The code permits the moisture content, volume of water per volume of the system, to range between 0.0 and 1.0. Typically values fall within a more narrow range, i.e., from 0.05 in very arid sites to 0.5 in saturated sites.

For the FD model, the moisture content may vary with position. The values for moisture content should be determined from detailed simulations of the flow around the facility. In assigning moisture content values, the user must specify a value for every control volume. If the moisture content values remain constant or change linearly between control volumes for a region of the modeled domain, automatic generation of the moisture content can be prescribed.

The DUSTIN code forces the user to enter the moisture content for each control volume (node), beginning with Node 1 and continuing sequentially until all nodes are specified. When entering this menu, existing values are printed in the form required as input by DUST and the user is asked if a change is desired. 


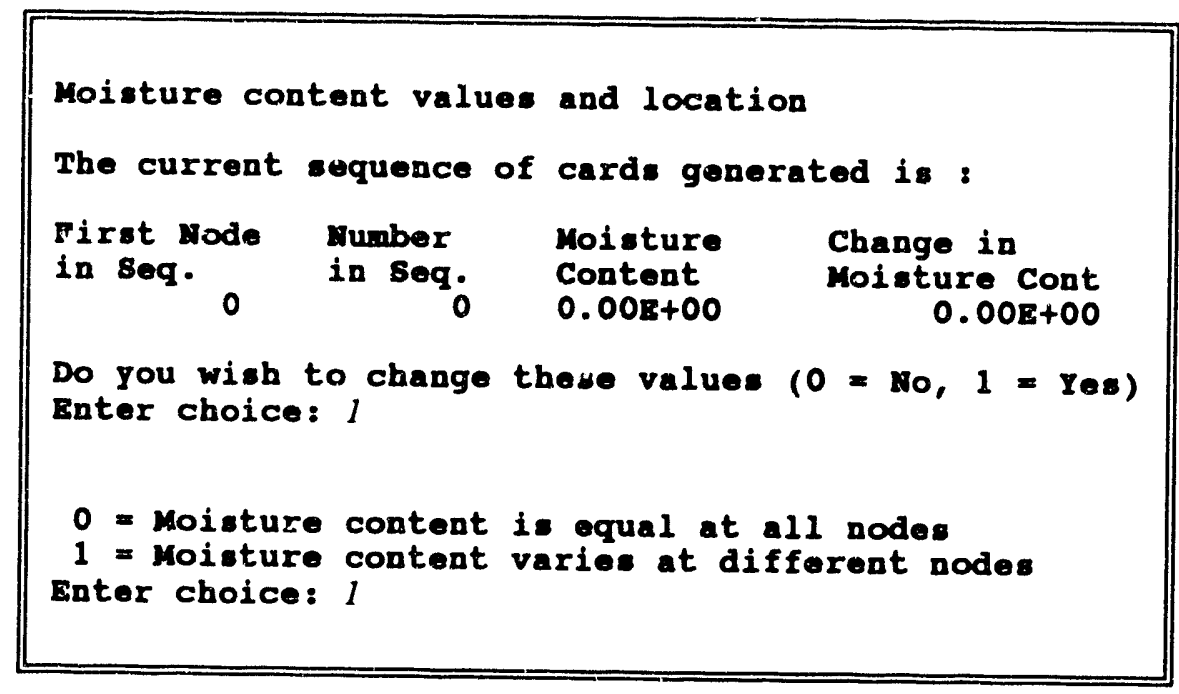

If the moisture content is constant ( 0 entered on the previous menu), the code asks for the value. If the moisture content changes with location, the following instructions are printed.

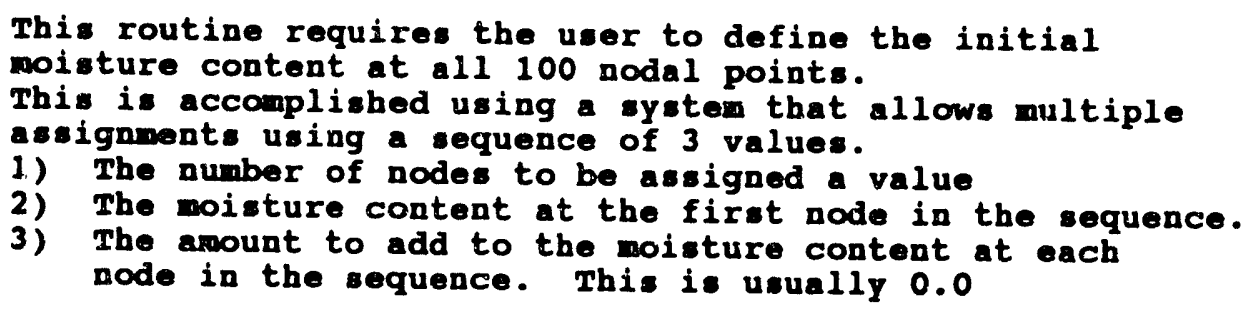

In the following example, the number of nodes has been specified to be 100 and the moisture content is set to 0.25 for the first 50 nodes and 0.3 for the last 50 nodes. 


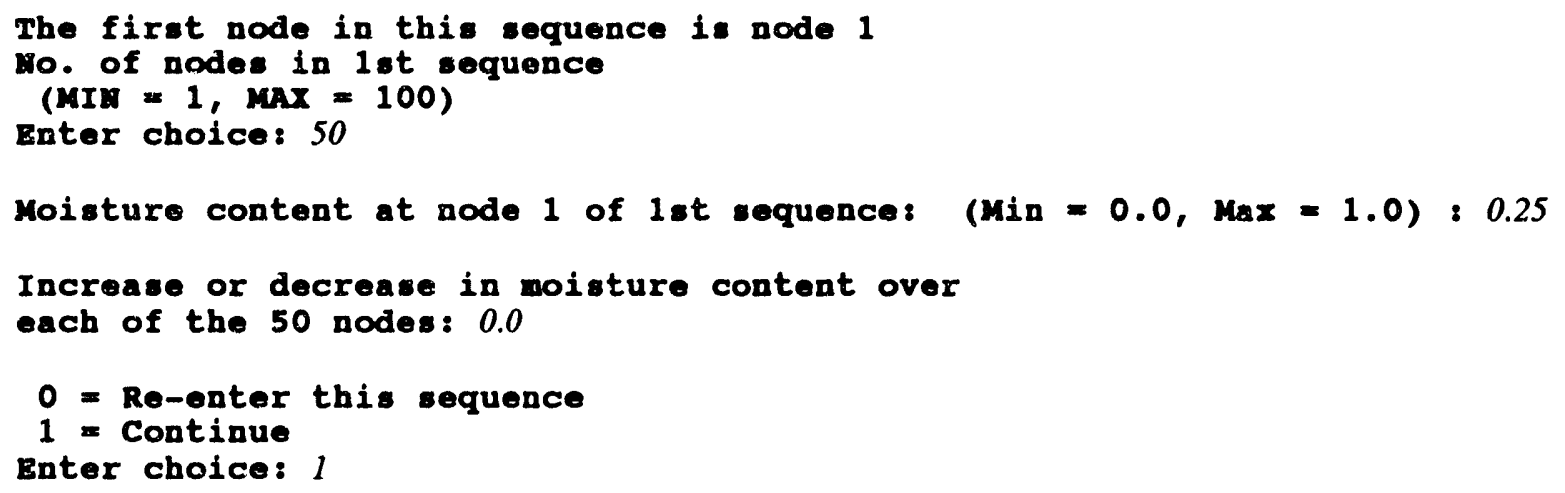

At this point, the code recognizes that only 50 of the 100 nodes have been given a value for moisture content. Therefore, it prints the instructions out and requests more input. Notice that the code begins this sequence at Node 51, the first undefined node.

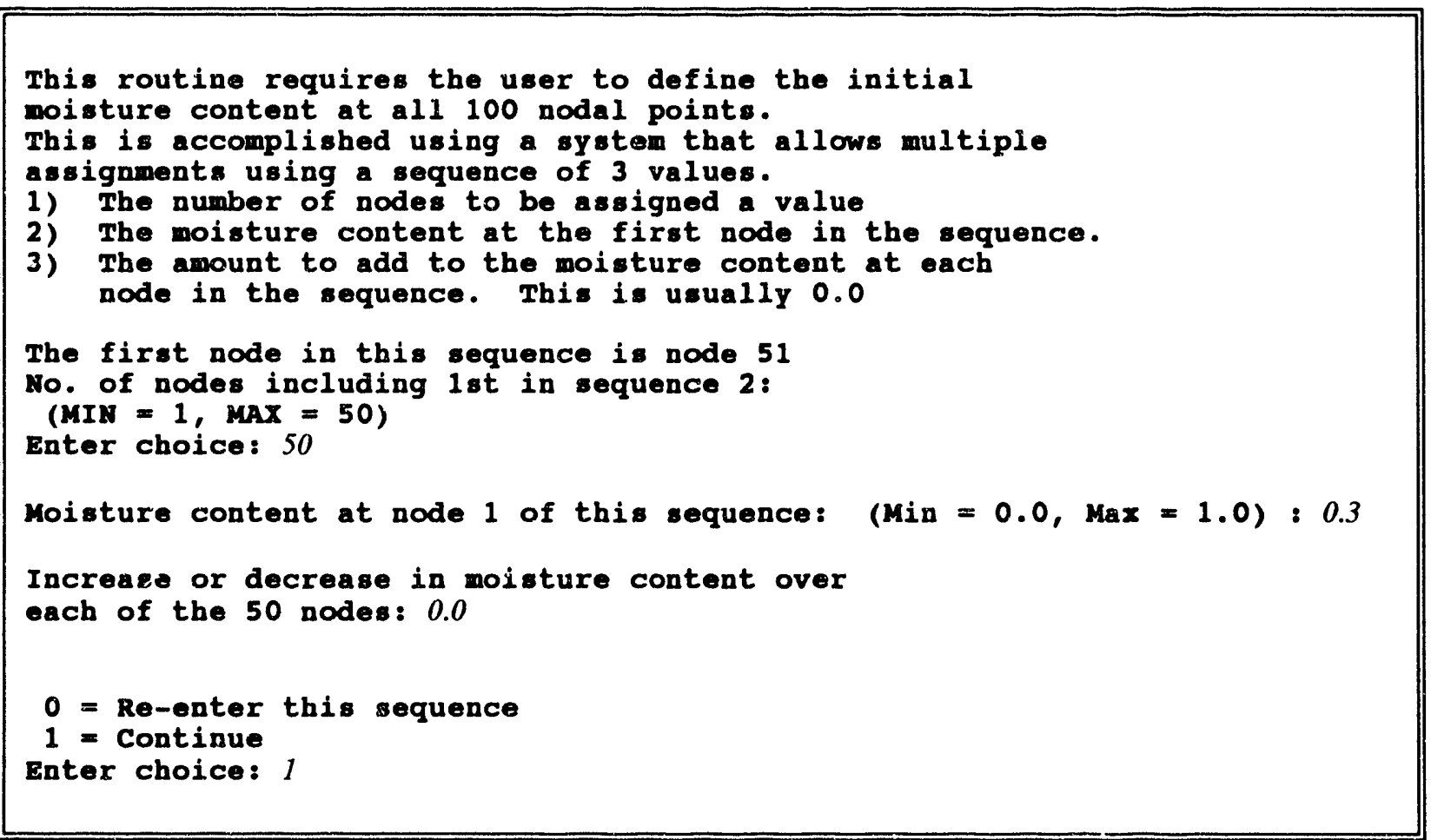

Upon specifying all moisture content values the code prints out the input cards needed by the DUST code. 


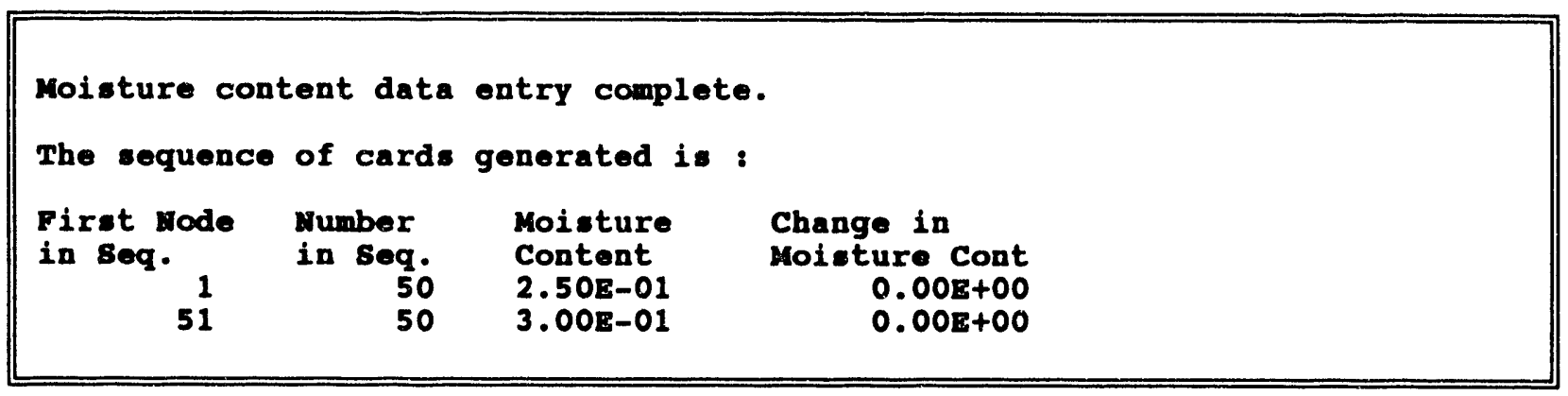

\subsection{Container Parameters}

The DUST computer program allows up to 300 different containers each with a unique time to failure. The MCMC model does not allow localized failure to occur. Therefore, it requires only the number of containers, the time to failure and location of each container. The FD model permits up to 20 different localized container failure rates to be applied to the containers. Therefore, it requires input on the rate parameters, and integer flags that specify which set of rate parameters should be used for each container. The container menu is:

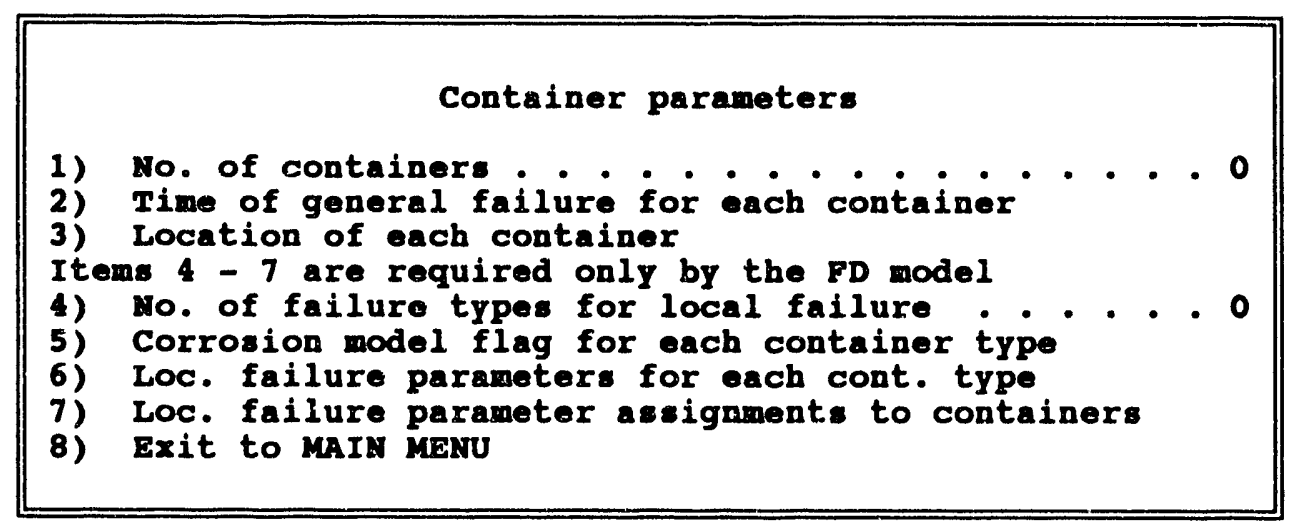

\subsubsection{Number of Containers}

This input variable provides the total number of mixing cells or control volumes that have a wasteform source.

\subsubsection{Time of General Failure}

Each container has a time of general failure after which the container no longer provides a barrier to release from the wasteform. These times should be estimated based on the material, thickness, and expected degradation rates. Information is available on corrosion rates for carbon and stainless steels in soil systems [Romanoff, 1957; Gerhold, 1981; Sullivan, 1989]. 
The failure time must be input for each container in units of years. Upon entering this submenu, the code prints the current values for failure times and allows the user to change each one of these times independently or set a series to a single value.

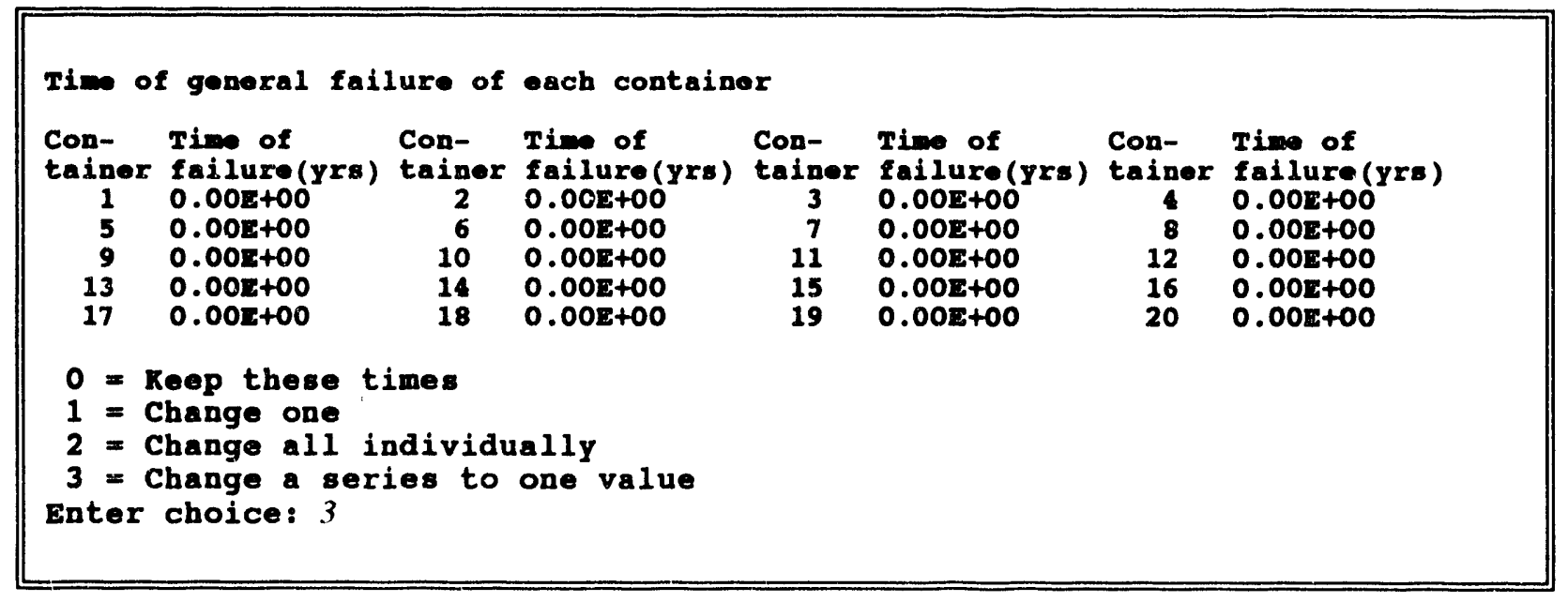

In changing a single value, the code asks for the node number and the failure time. When changing a series to one value, the following instructions are provided on the screen when running DUSTIN. In the example, the user has previously specified that there are 20 containers and then sets the failure time of the first 10 containers at 10 years and the last 10 containers at 20 years. This is achieved through input of three values: a) the value for the first container in this sequence; $b$ ) the total number of containers in the sequence; and c) the failure time. After a sequence has been completed, the current failure times are printed and the user is asked if more changes are required.

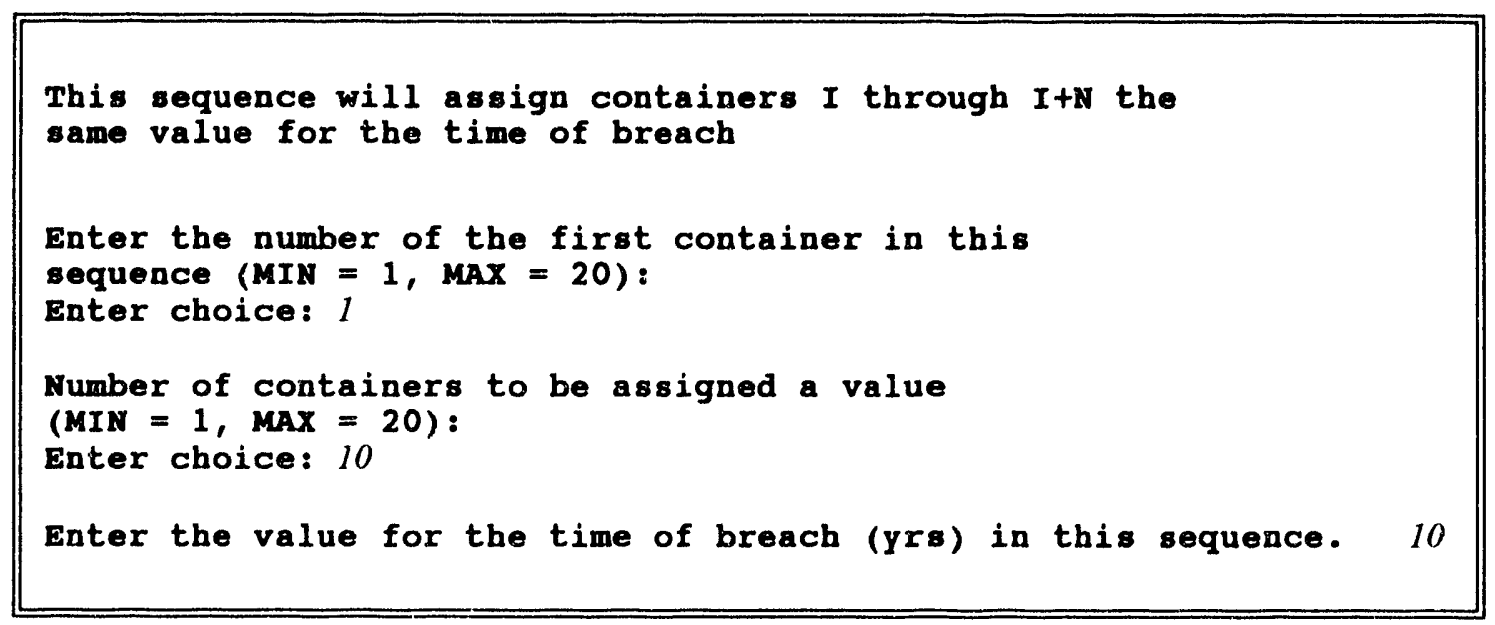


The process was continued for the remaining containers. Output of the current values for failure times follows:

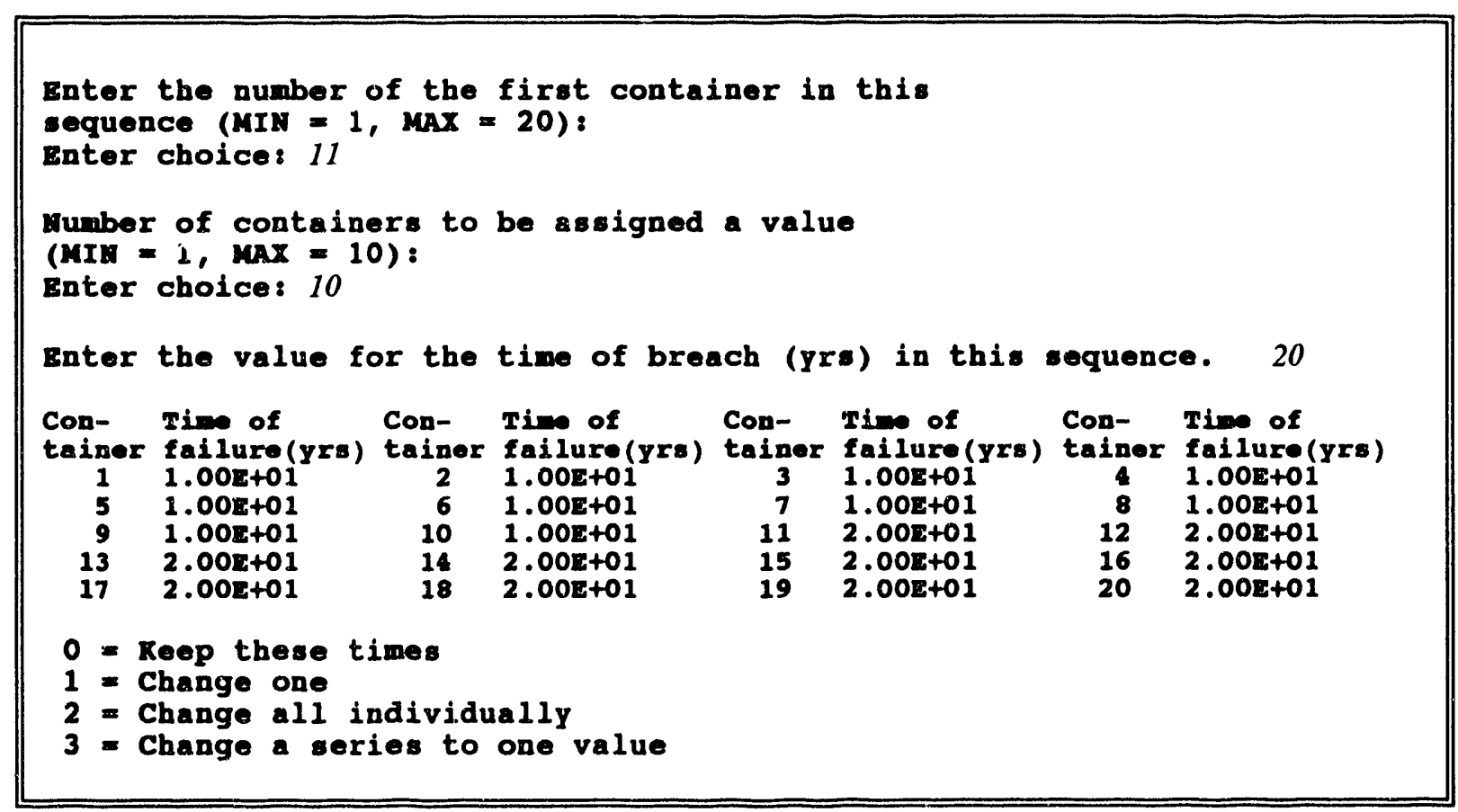

\subsubsection{Location of the Containers}

Each container must be assigned to a unique location. This is accomplished by DUS'TIN in a similar fashion as specifying the failurc times. The user is permitted to change each value independently, or change a sequence of values. If a sequence of values are to be created, four values are needed: a) the number of the first container in the sequence; b) the location (cell number) of the first member of the sequence; $c$ ) the distance between (number of cells) adjacent containers; and d) the number of values to be assigned in the sequence. The default value for container location is zero. Failure to define this number will cause the DUST code to fail.

The equation used to generate this sequence is presented in the menu as shown below. In the example problem, the user has specified that the first container is in node 20 and the remaining containers are in the 19 adjacent cells. The DUSTIN code requires that each container location be defined. If the user has not defined all locations the code returns to the location specification menu and requires further input. 


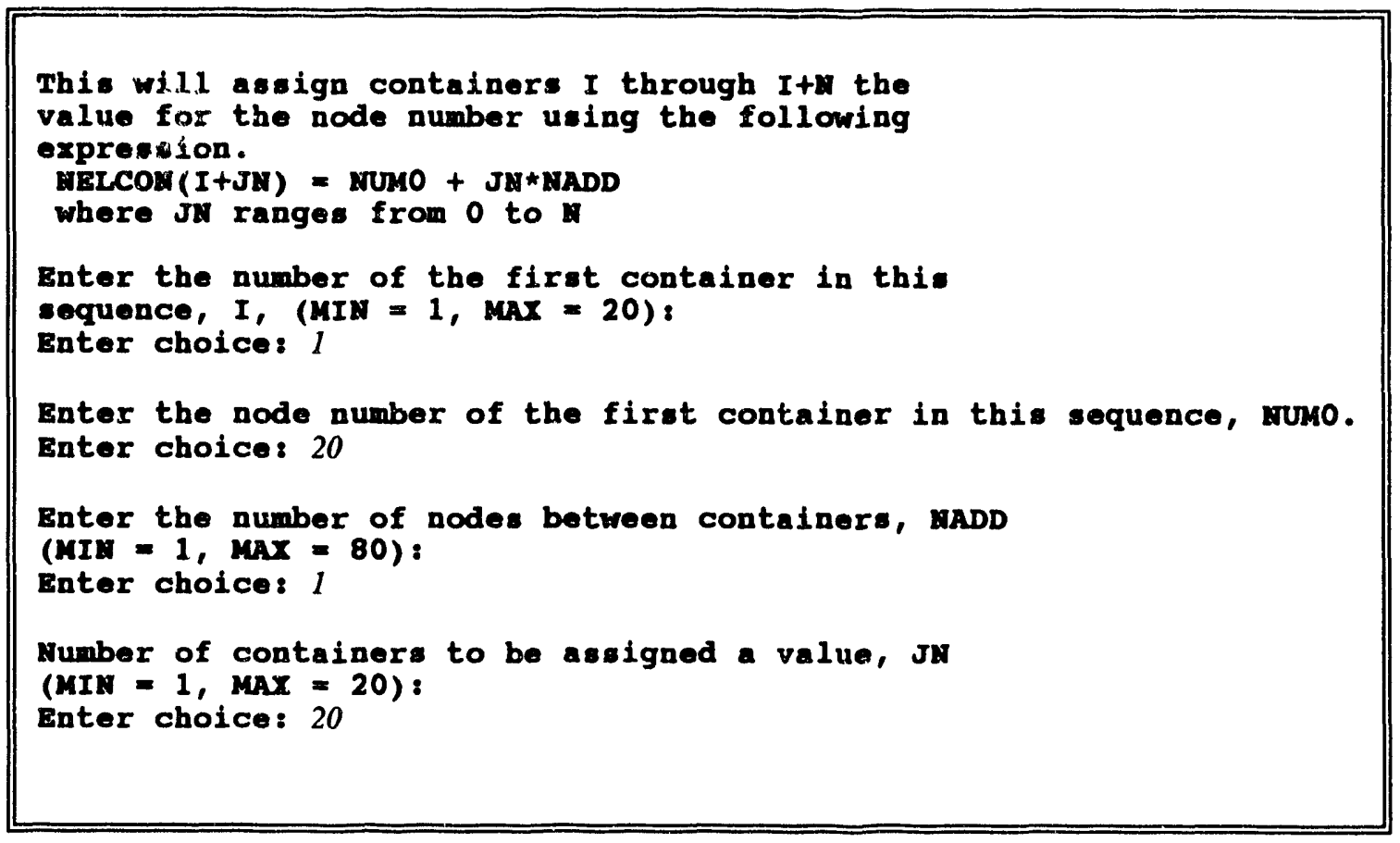

Upon completion of the input, the values are translated into cell (node) numbers and the results are printed for review.

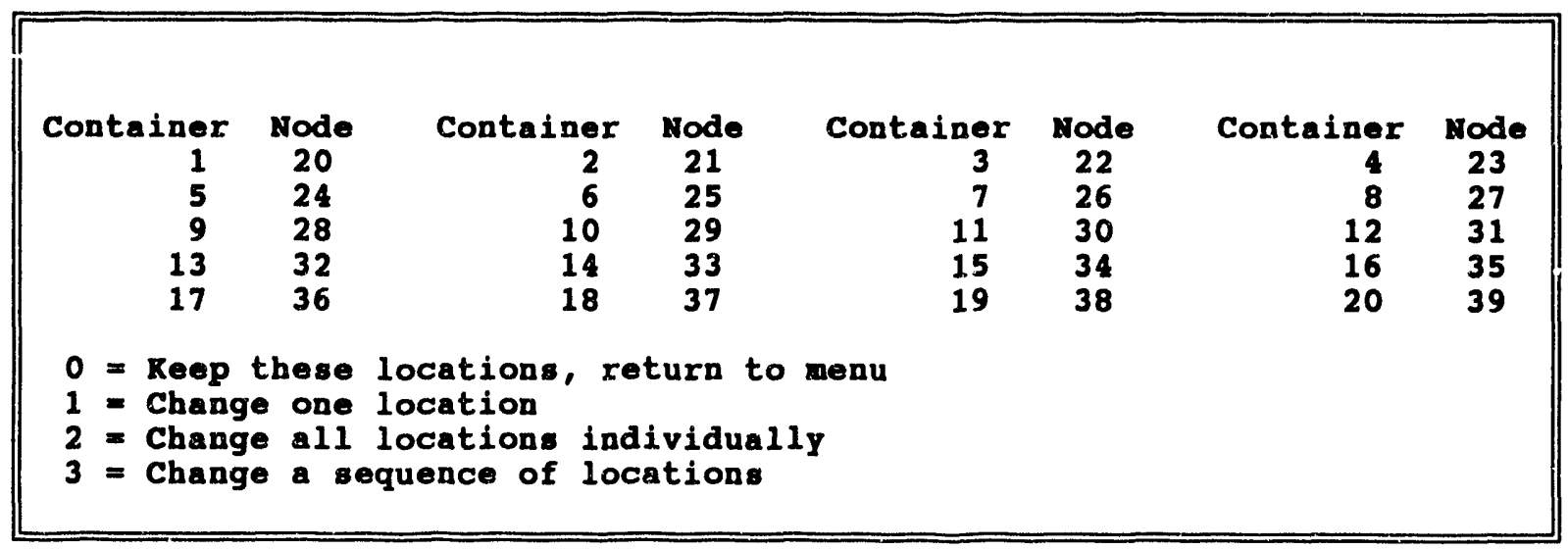

\subsubsection{Number of Failure Types (FD)}

The FD model permits localized failure. The data in the model has been specialized to pitting corrosion but it may be generalized to provide an early localized failure for any process. Up to 20 different localized failure rates may be specified. If localized failure is not desired leave this value equal to the default value, 0 . 


\subsubsection{Corrosion Model Flag}

All containers must have a specified time to failure. However, each container type may also fail due to local corrosion. The choice is specified through setting this flag to 1 for local corrosion. This is used only if the number of failure types is non-zero. The structure of the input routine for this value is identical to that for specifying the time to failure and will not be repeated. This is useful when the user desires to model a portion of the containers failing by local corrosion and the remainder by a general failure time. The code user could specify more than one container type and require that one container type does not undergo local corrosion by setting this flag to zero.

\subsubsection{Localized Failure Parameters}

The model for localized failure has been presented in Section 2.4.2. The model requires six parameters (the number of penetrating pits per container, the area of the container, the area scaling factor, the container thickness, and the two empirically determined rate parameters used to describe pit growth). Suggested values for these parameters are presented in Appendix C. The DUSTIN code permits the user to change every single variable independently through the use of the submenus. Upon entering this submenu, the following instructions are printed.

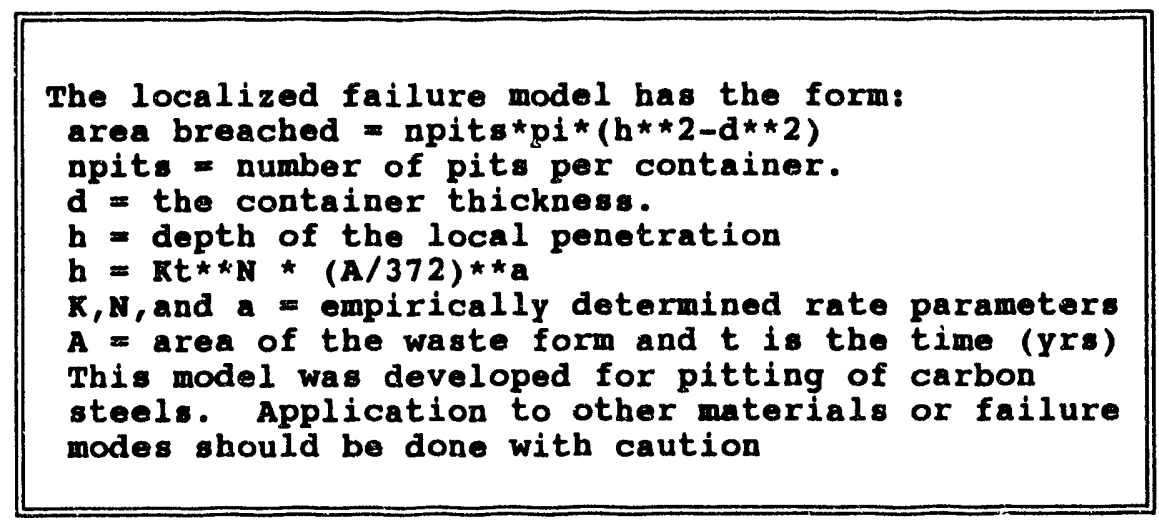

The current values for these parameters are then printed and the user is asked if a change is requested.

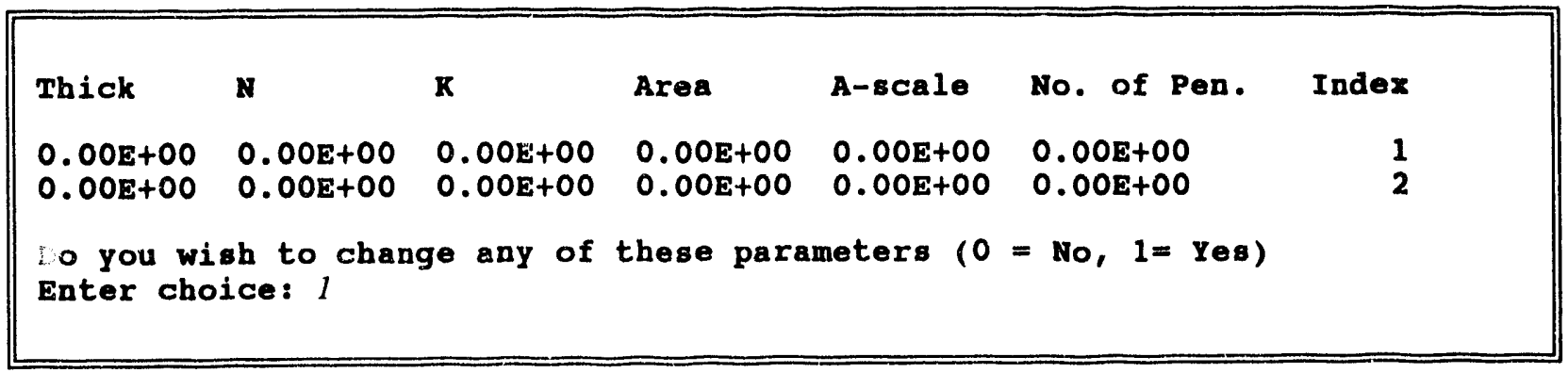


The code then prints out the container types that require localized corrosion parameters and asks which container type will receive changes.

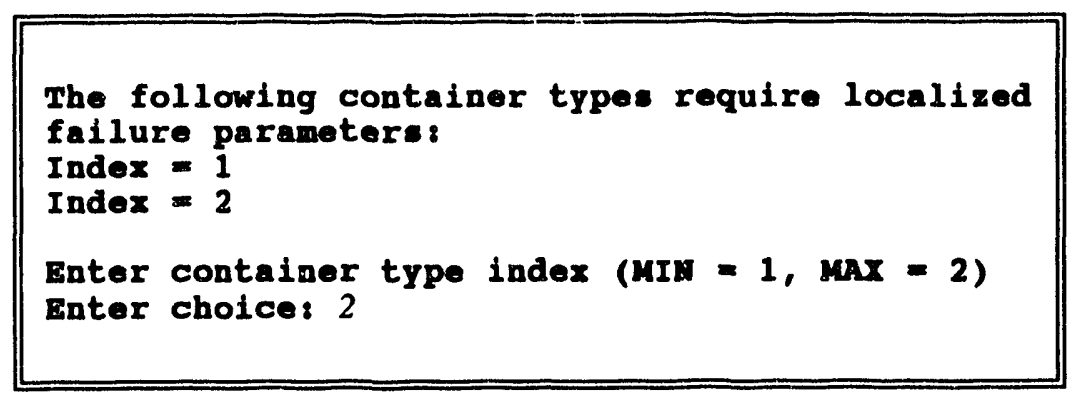

Here container 2 receives the changes. The code then prints out information on the input variables in the failure parameter menu. A full discussion of these variables can be found in [Sullivan, 1989].

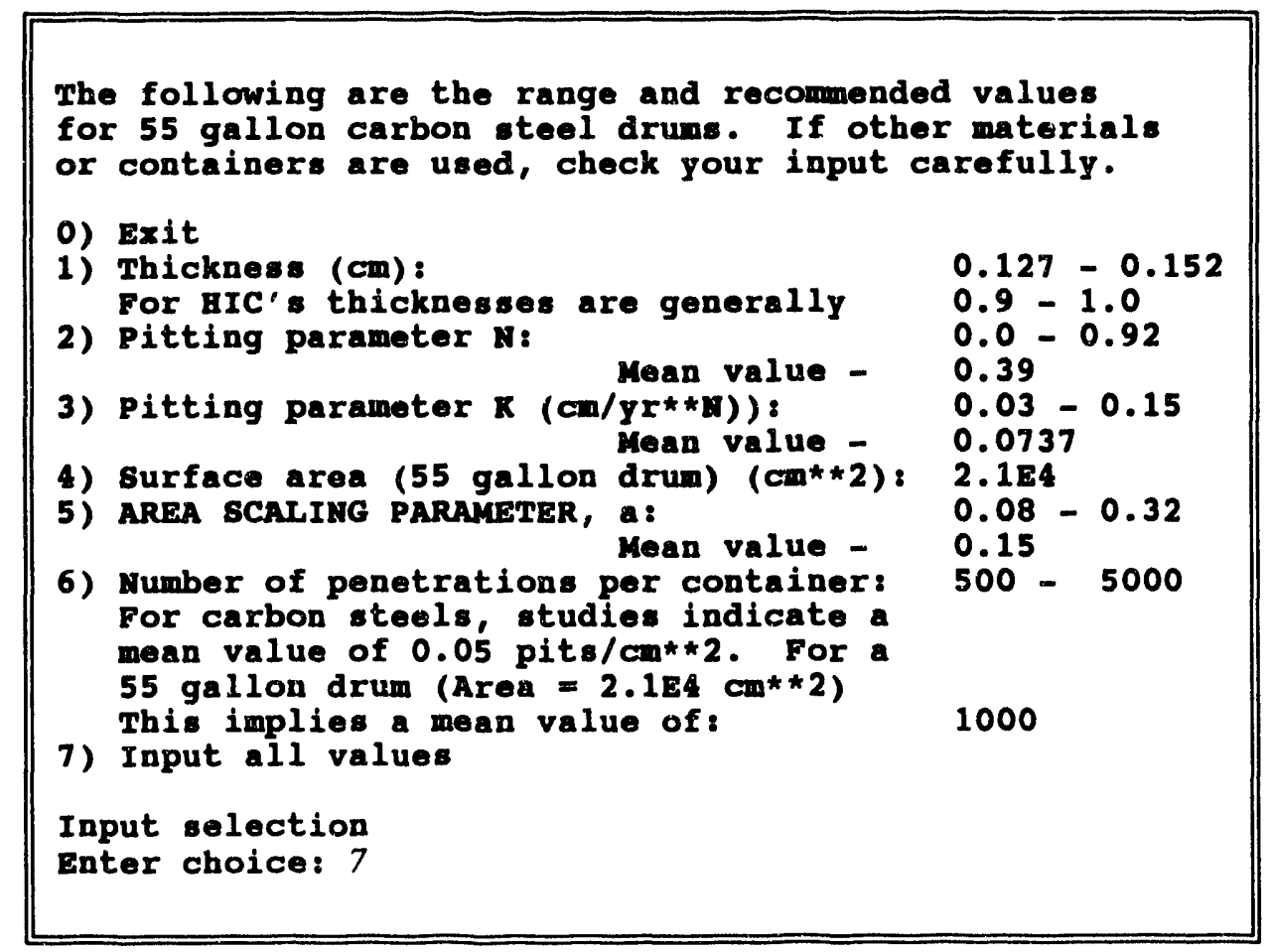

In this case, all parameters will be changed. The code then prints the following and requests the input for all parameters on one line separated by spaces. 


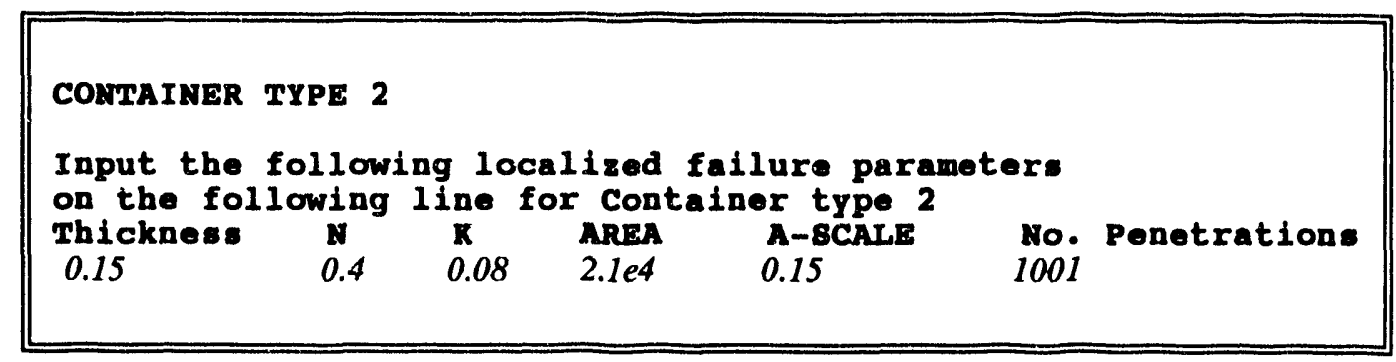

If the change was to be made only to a single parameter, the code prints the parameter name and asks for the input value. It then returns to the parameter menu and asks if another parameter is to be changed. In the example, the code asks if the user wants to continue data entry. If further input is requested, the code asks which material is to receive changes. If input of local failure parameters is complete, control is returned to the container parameter menu (i.e., the menu containing information on number of containers, time of failure, etc.). Upon returning to that menu, the values for the rate parameters are printed.

\begin{tabular}{|llllllr||}
\hline Thick & $\mathrm{N}$ & $\mathrm{K}$ & Area & A-scale & No. of Pen. & Index \\
$1.50 \mathrm{E}-01$ & $3.90 \mathrm{E}-01$ & $1.00 \mathrm{E}-01$ & $2.10 \mathrm{E}+04$ & $1.50 \mathrm{E}-01$ & $1.00 \mathrm{E}+03$ & 1 \\
$1.50 \mathrm{E}-01$ & $4.00 \mathrm{E}-01$ & $\mathbf{8 . 0 0 E - 0 2}$ & $2.10 \mathrm{E}+04$ & $1.50 \mathrm{E}-01$ & $1.00 \mathrm{E}+03$ & 2 \\
\hline
\end{tabular}

\subsubsection{Assignment of Localized Failure Parameters to Each Container}

If more than one set of container types is requested in Menu 8.4 , the user must specify the container type for each container. This assigns local container failure parameters to each container. The procedure is similar to that used in specifying times to failure, the user can change any single container type or a sequence of container types. Upon entering this menu, the code prints out the existing values and requires reassignment to non-ze $\mathrm{i}_{\mathrm{O}} \mathrm{v}$ values. 


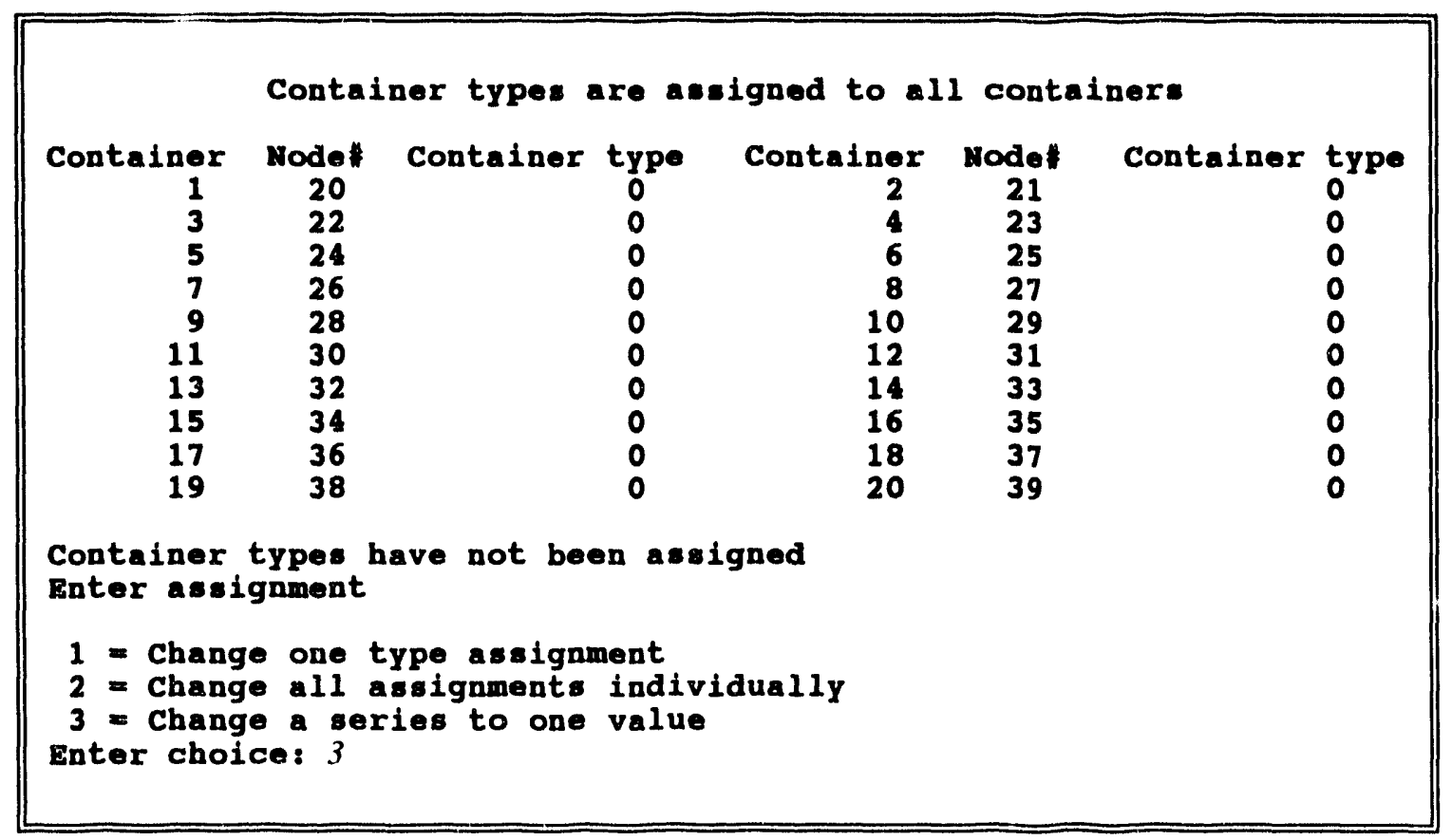

To reassign a series of container types three input variables must be supplied, the container number of the first container in the series, the number of containers in the series, and the container type for the series. In this example, containers $1-10$ are given type 1 failure parameters and containers $11-20$ are specified as type 2 containers.

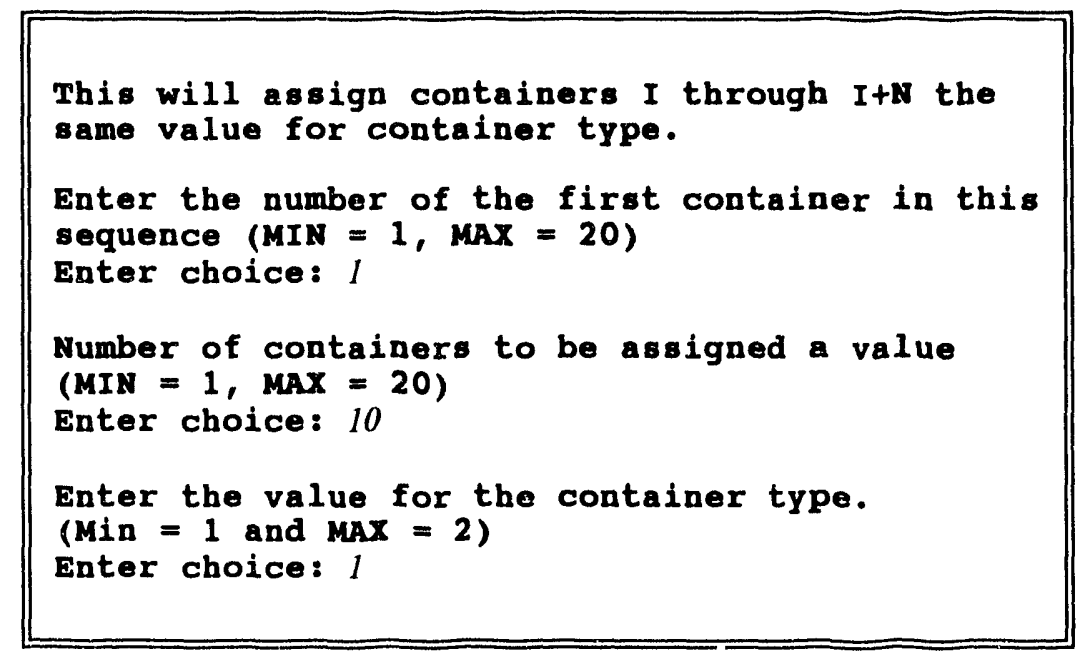

At this point, only 10 of the 20 containers have been reassigned. The DUSTIN code auromatically asks for a continuation until all 20 containers are assigned a non-zero value. 


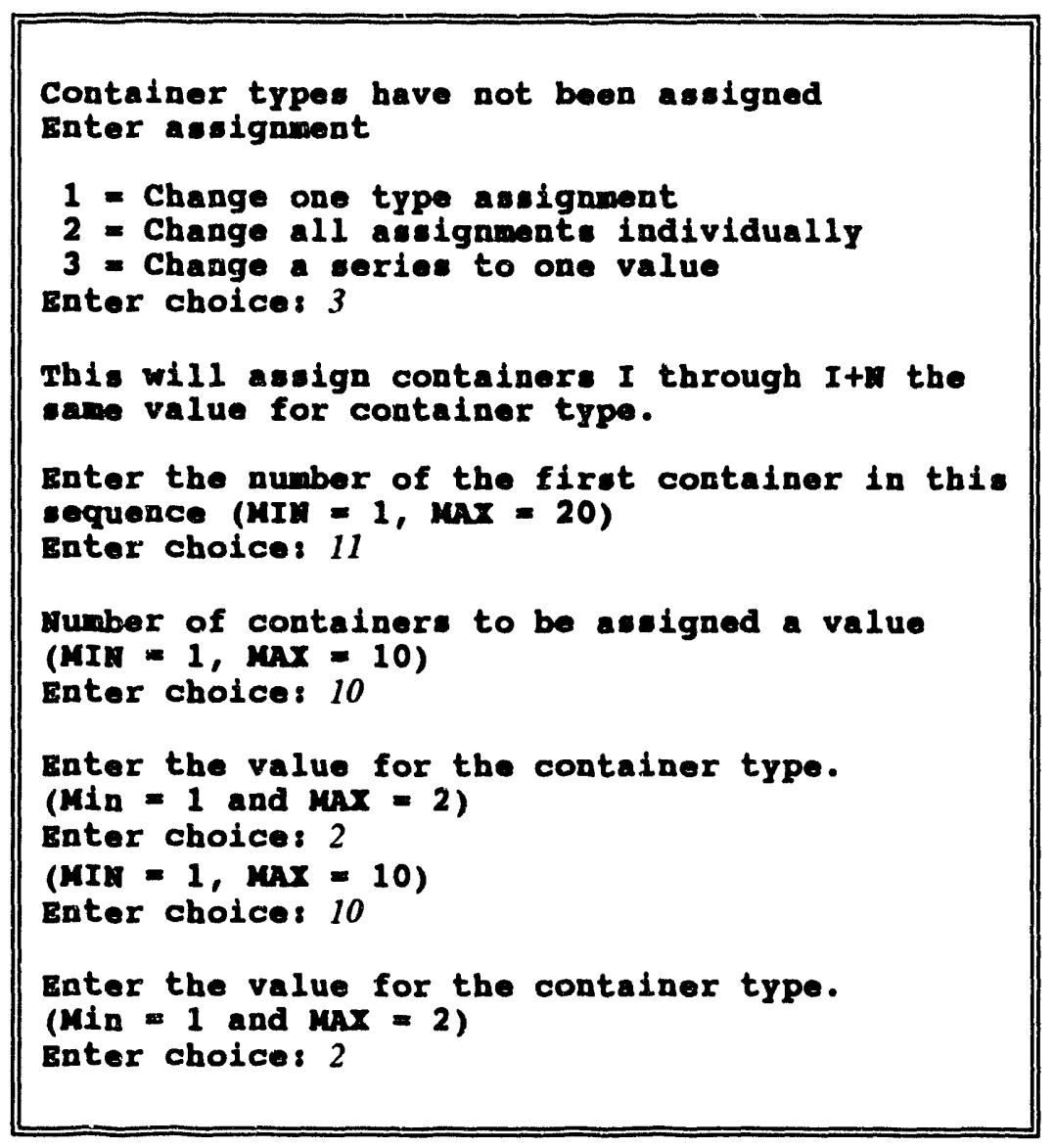

After the container assignments are complete, the values are printed for review.

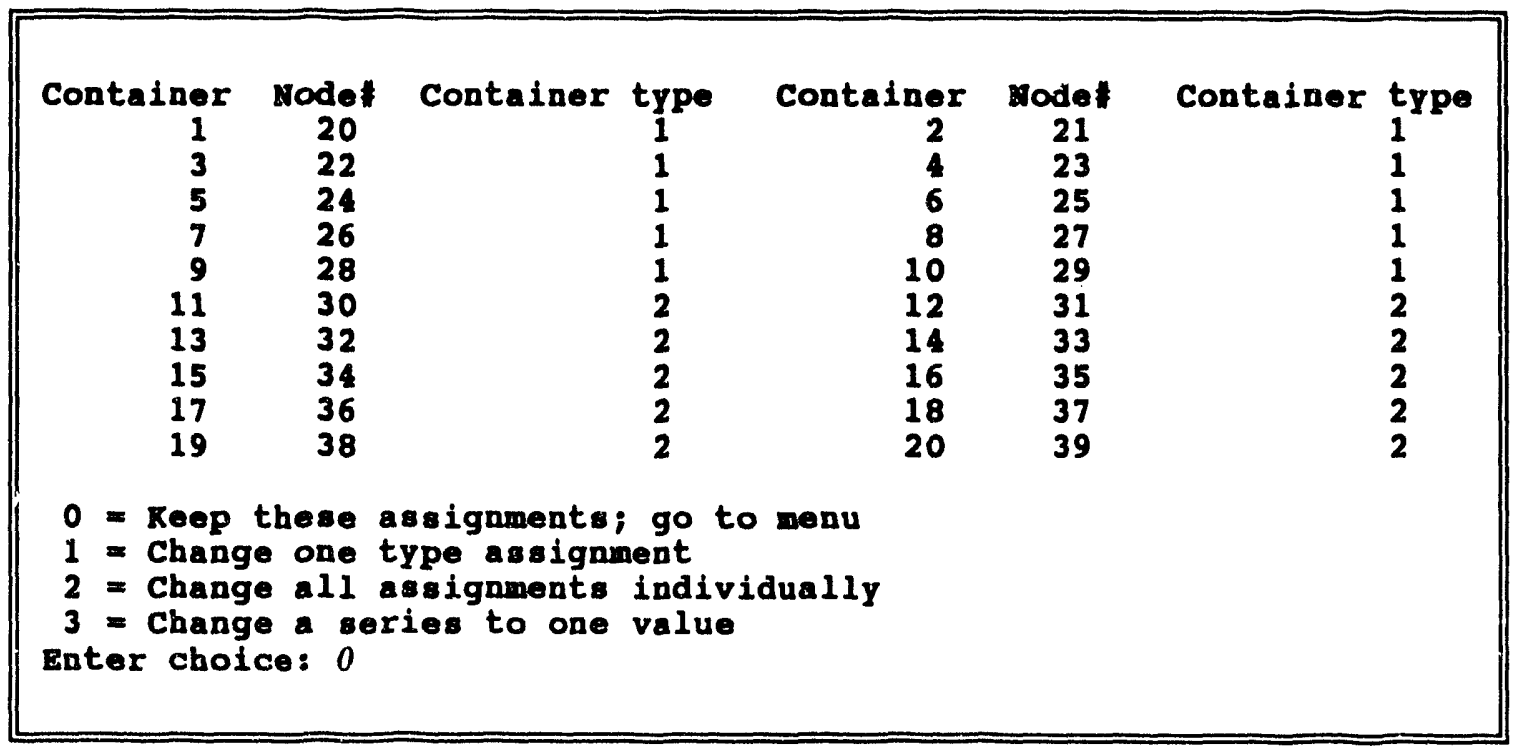




\subsection{Wasteform Parameters}

The DUST computer code simulates a wasteform in every control volume (or mixing cell) that has a container (the maximum number of containers is 300). Each wasteform is permitted to have a unique inventory. However, the code permits only twenty different wasteform types to be modeled. Each wasteform type is represented by a unique set of release rate parameters.

Again, the choice of the transport model has a large impact on the amount and type of input required. The MCMC model allows an exponentially decaying release rate or a rinse release. The FD model allows these two release mechanisms plus a diffusion release mechanism. Diffusive releases are dependent on the geometry and dimensions of the wasteform. This requires additional input. Either rectangular or cylindrical geometry can be modeled. The models have been presented in detail in Section 2.5 and will not be repeated.

The wasteform parameters menu is:

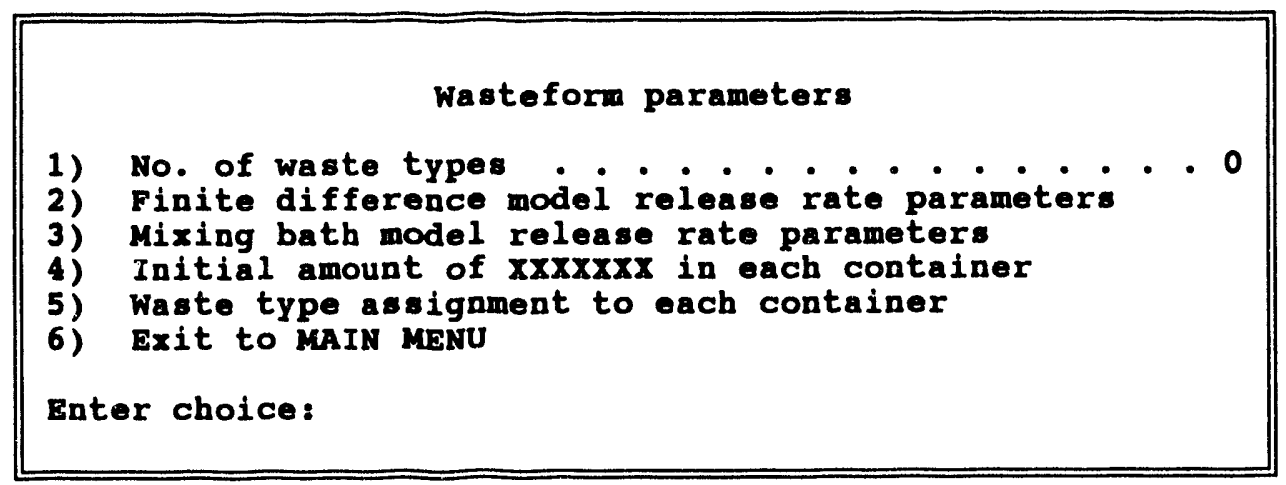

\subsubsection{Number of Waste Types}

The number of waste types is the number of wasteforms having unique release rate parameters. The maximum value permitted in DUST is 20.

\subsubsection{Finite Difference Model Release Rate Parameters} is printed.

Upon requesting to alter the finite difference release rate parameters, the following menu 


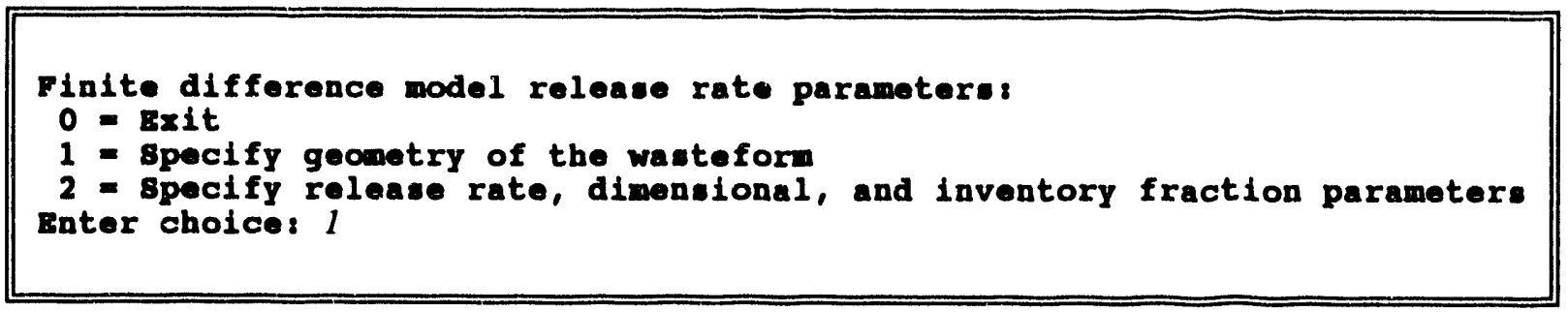

For diffusion controlled release the code contains analytical solutions for release from cylindrical or rectangular wasteforms [Section 2.5.3]. Therefore, the geometry must be specified. The default choice is cylindrical geometry. If diffusional release from a rectangular wasteform is modeled, the geometry flags need to be redefined.

If the geometry is to be redefined, the code prints out existing values for the flags, defines the flags, and asks if a change is desired.

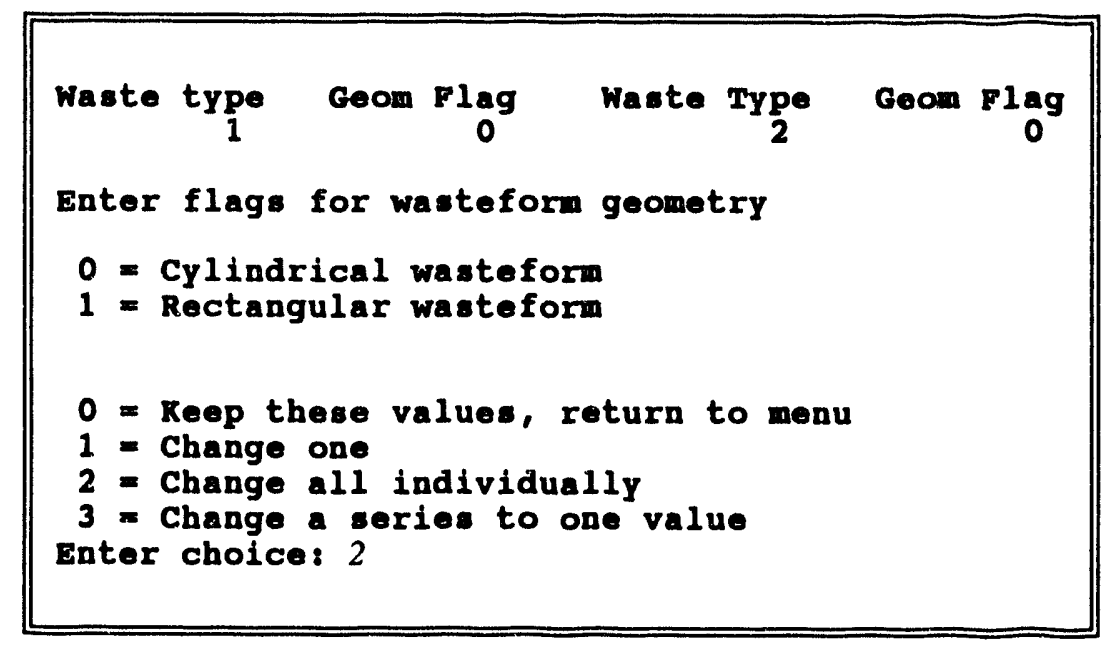

In this example, the user has decided to change each flag individually. The code then prompts the user for the flag values.

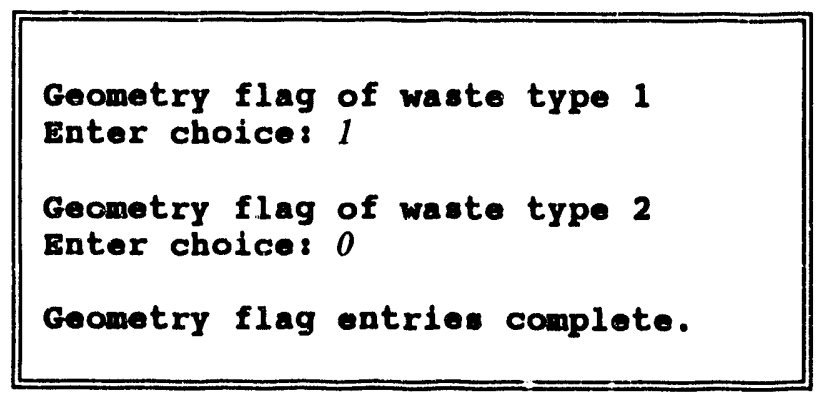


At this point, the code prints the values for the flags and provides the user with an opportunity to make further changes or return to the menu which accesses the release rate parameter menu below. At this time, the example below indicates that release rate parameters require definition.

Pinite difference model release rate parameters

0 - Exit

1 = specify geometry of the wasteform

2 = specify release rate, dimensional, and inventory fraction parameter Enter choice: 2

The release rate parameter menu follows. Again, the user can change all parameters independently through the menu. Upon entering this menu, the current values are printed and the user is asked if changes are desired for one or all waste types. In this example, two waste types (Menu 9.1) have been requested and the FD transport model is specified.

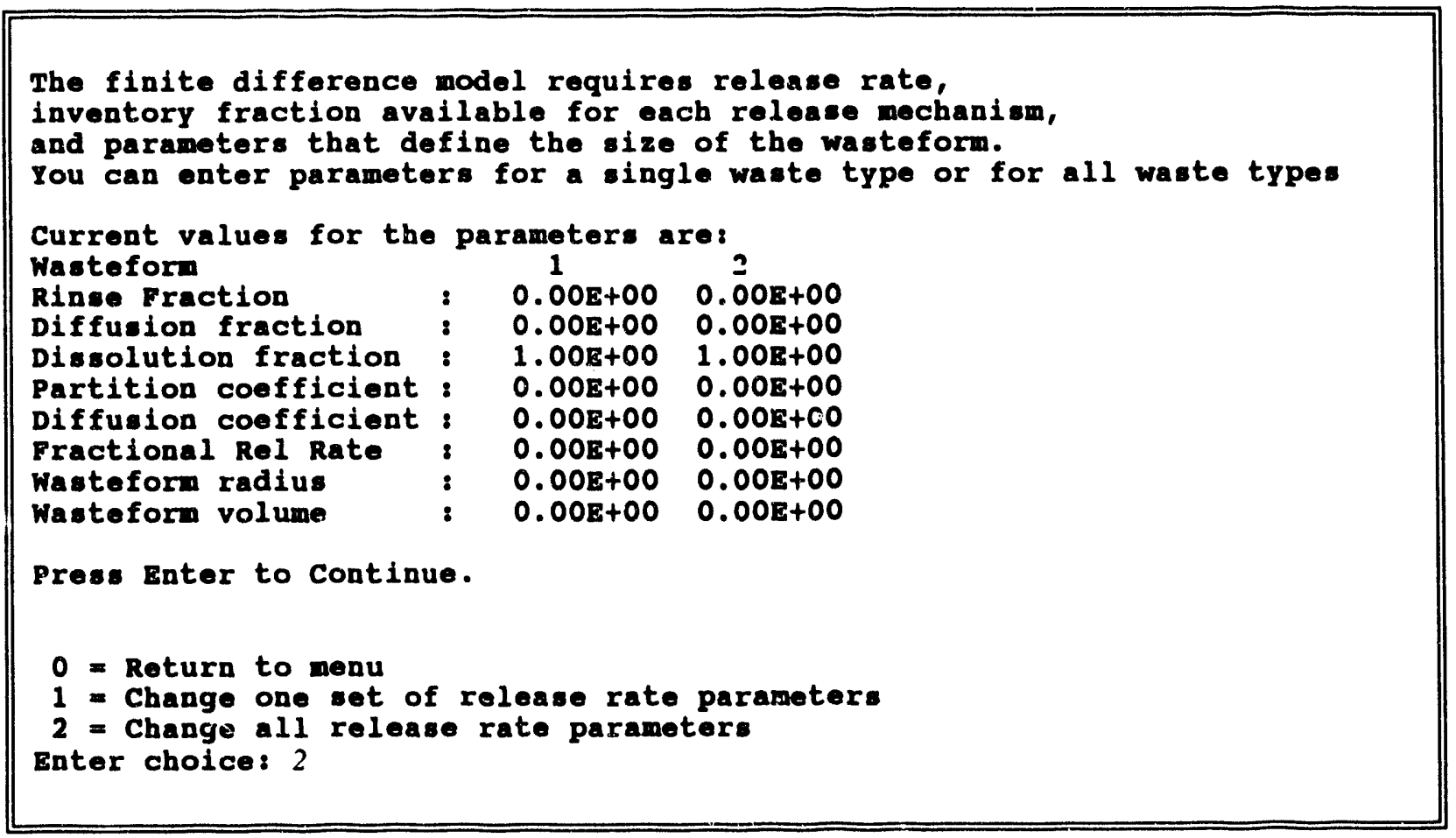

The code will cycle through all of the different waste types asking for changes to the release parameters. In this example, waste type 1 has been defined and the code asks for changes to waste type 2 . 


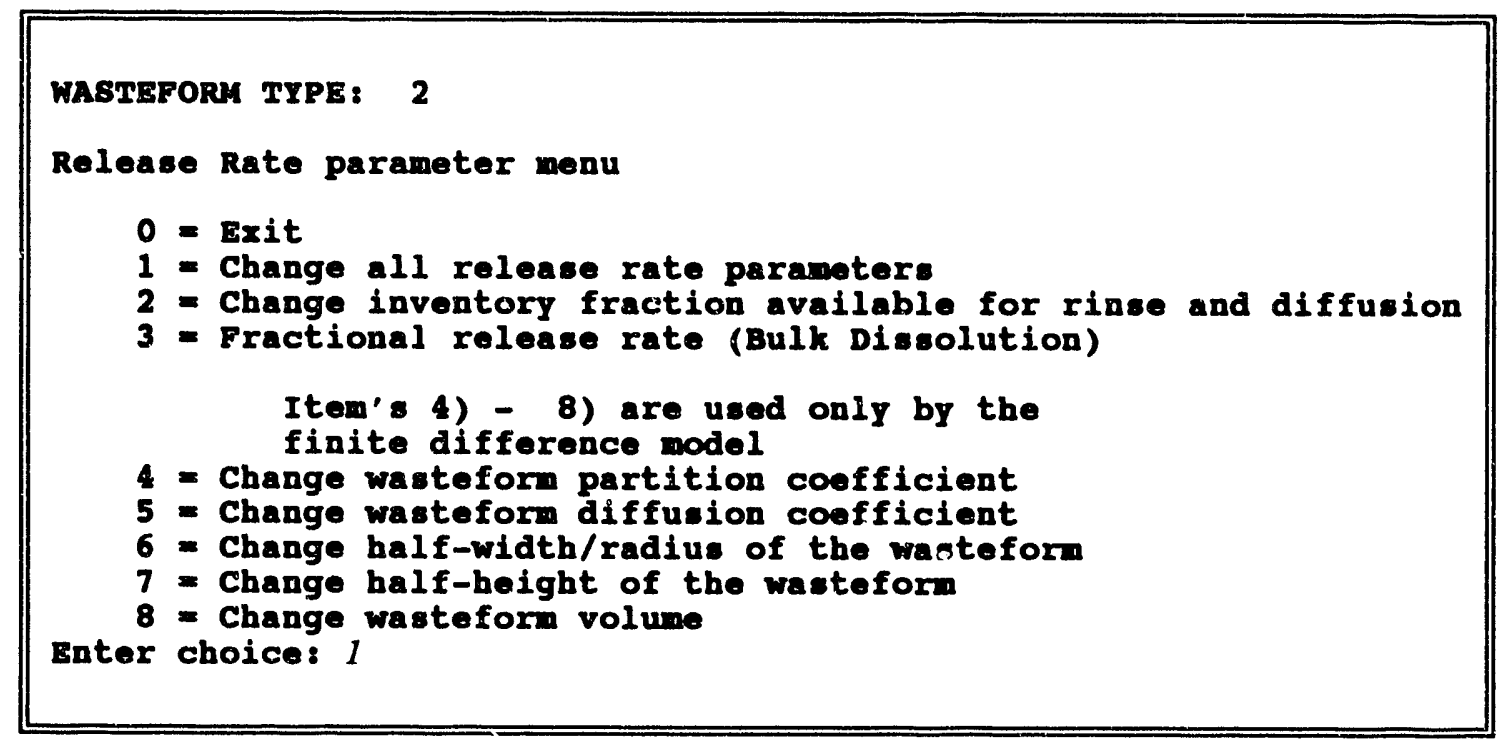

The first time through this menu it is necessary to define all of the parameters. For the finite difference model, there are three release mechanisms: rinse, diffusion, and uniform. Each of these is independent. The amount of mass available for release by each mechanism is the total mass in the wasteform (Menu 9.5) multiplied by the fraction of mass available for each mechanism. These are input as the fraction available for rinse and the fraction available for diffusion. The fraction available for uniform release is determined by requiring that all three sum to 1 .

Item 3 on the menu, the fractional release rate, is the fraction of the uniform release fraction that is released per year. For example, if a wasteform had an initial inventory of 1 Curie and $10 \%$ of the inventory was available for uniform release, a fractional release rate of 0.05 per year would release $0.005 \mathrm{Ci} / \mathrm{yr}$ (this value would be adjusted to account for decay) due to uniform release. Additional releases would occur due to the diffusion and rinse mechanisms. This parameter is also used by the MCMC model.

Item 4, the wasteform partition coefficient, can be used to represent control of release by reversible chemical interactions with the wasteform, for example sorption on dewatered ion exchange resins. If this value is zero, the entire rinse mass (total mass multiplied by rinse fraction) is released into solution upon breach of the container subject to solubility limits. If the partition coefficient is non-zero, releases from this wasteform can be negative. The model performs a mass balance and determines the distribution of contaminants subject to the partition coefficient. Therefore, contaminants in solution may be removed causing a negative release for this wasteform. When using the partition coefficient the code user must be extremely careful so as not to account for sorption effects twice as there is also a distribution coefficient in the transport model. This is discussed in detail in Section 2.5.2. 
Item 5, wasteform diffusion coefficient, is used when the diffusive release fraction is nonzero. The code has analytical solutions for finite-sized cylindrical and rectangular wasteforms, see Section 2.5.3. Values for the diffusion coefficient depend on the waste stream and solidification agent. The Technical Position on Wasteform [Lohaus, 1991] requires that the diffusion coefficient be smaller than $10^{-6} \mathrm{~cm}^{2} / \mathrm{s}$ for solidified wastes. A collection of diffusion coefficient values is presented in [Sullivan, 1989].

Diffusive releases depend strongly on the wasteform dimensions. For a fixed value of the diffusion coefficient, larger wasteforms will have a lower cumulative fractional release as compared to smaller ones. Therefore, it is crucial to input the proper dimensions. In many cases, in order to improve the numerical accuracy of the FD model, it may be necessary to model a single wasteform using several control volumes. In this case, the wasteform dimensions must be input as the true dimensions. To properly model the total release, the wasteform mass should be evenly divided among all control volumes representing the wasteform. For example, if 10 control volumes were used to model a cylindrical wasteform having a height of $50 \mathrm{~cm}$, a radius of $25 \mathrm{~cm}$ and with an initial inventory of 40 curies, the proper way to model this would define 10 wasteforms all with the same release mechanisms, i.e., all the same wasteform type. The radius of this wasteform type is $25 \mathrm{~cm}$ and the volume is $9.82 \mathrm{E} 4 \mathrm{~cm}^{2}$. The initial inventory for each of these 10 wasteforms is 4 curies.

For rinse and uniform release, the dimensions are unimportant in estimating wasteform release. Therefore, if a wasteform is divided, the initial mass of the contaminant should be divided uniformly between the control volumes.

Item 6, is a dual purpose variable. For cylindrical wasteforms, it is the radius. For rectangular wasteforms it is the half-width. This variable is only used for predicting diffusion release. The analytical release models require the dimensions of the wasteform. In DUST, these are specified by supplying the radius and volume for cylindrical wasteforms. The half-width, half-height, and the volume combine to define the geometry of rectangular wasteforms.

Item 7 , is the half-height of a rectangular wasteform. This variable is not used for cylindrical wasteforms.

Item 8 , is the volume of the wasteform.

If the mass fraction available for diffus on is zero for a waste type, items 5 - 8 are not required as input.

The following example requests the properties for waste type 2 which was previously defined as having cylindrical geometry. The user has requested to change all variables. The code prompts the user for each variable and gives the required units. 


\begin{tabular}{|c|}
\hline $\begin{array}{l}\text { WASTEFORM TYPE: } 2 \\
\text { Fraction of waste's inventory available for SURFACE RINSE: } 0.1 \\
\text { Fraction of waste's inventory available for DIFFUsiow: } 0.8 \\
\text { Fractional Release Rate }(1 / y r): 1 e-3 \\
\text { Wasteform partition coefficient }(\mathrm{cm} * * 3 / \mathrm{gm}): 0.0 \\
\text { Diffusion coefficient inside wasteform }(\mathrm{cm} * \star 2 / 8): 1 e-9 \\
\text { Radius of cylindrical waste (cm): } 25 \\
\text { Volume of wasteform (cm^3): } 1.67 e 5 \\
\text { Wasteform type parameters complete. }\end{array}$ \\
\hline
\end{tabular}

Upon completing the input routine, the code prints out the current values for the release rate parameters and asks if further changes are requested.

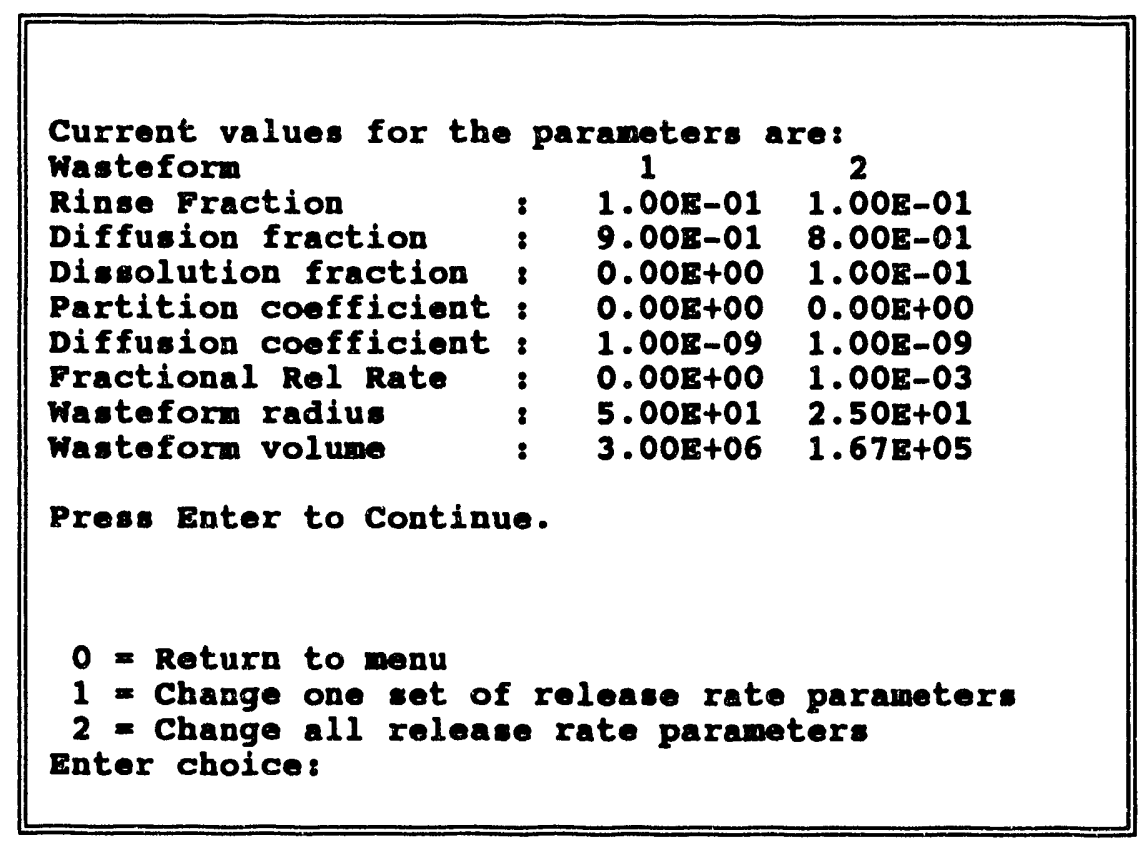

\subsubsection{Mixing Cell Model Release Rate Parameters}

The MCMC model permits only two release mechanisms: rinse and uniform release. In this rinse model, there is no wasteform partitioning coefficient. Upon container failure, all mass 
is released into solution. If the soil distribution coefficient is non-zero, the mass is distributed between the soil and water instantaneously. The uniform release parameter has the same definition as in the FD model. Again, the release rate for this model decreases exponentially due to radioactive decay.

Two release rate parameters, the fraction available for rinse and the fractional release rate, completely describe the release for the MCMC model. The input routine is similar to that for the FD model. When using the Release Rate Parameter Menu shown above, the code prevents the user from defining variables that are not needed for this model.

\subsubsection{Initial Inventory}

The user must supply the initial inventory for each waste container. The units for inventory are specified by menu item 1.3. Input can be cione for each individual container or a series of wasteforms may be assigned an identical inventory. In the following example, the user has previously requested 20 waste containers (Menu 8.1) with the inventory units of curies. The code prints the current inventory (all zero by default) and asks for changes. In this case, a series of inventories will be set to a single value.

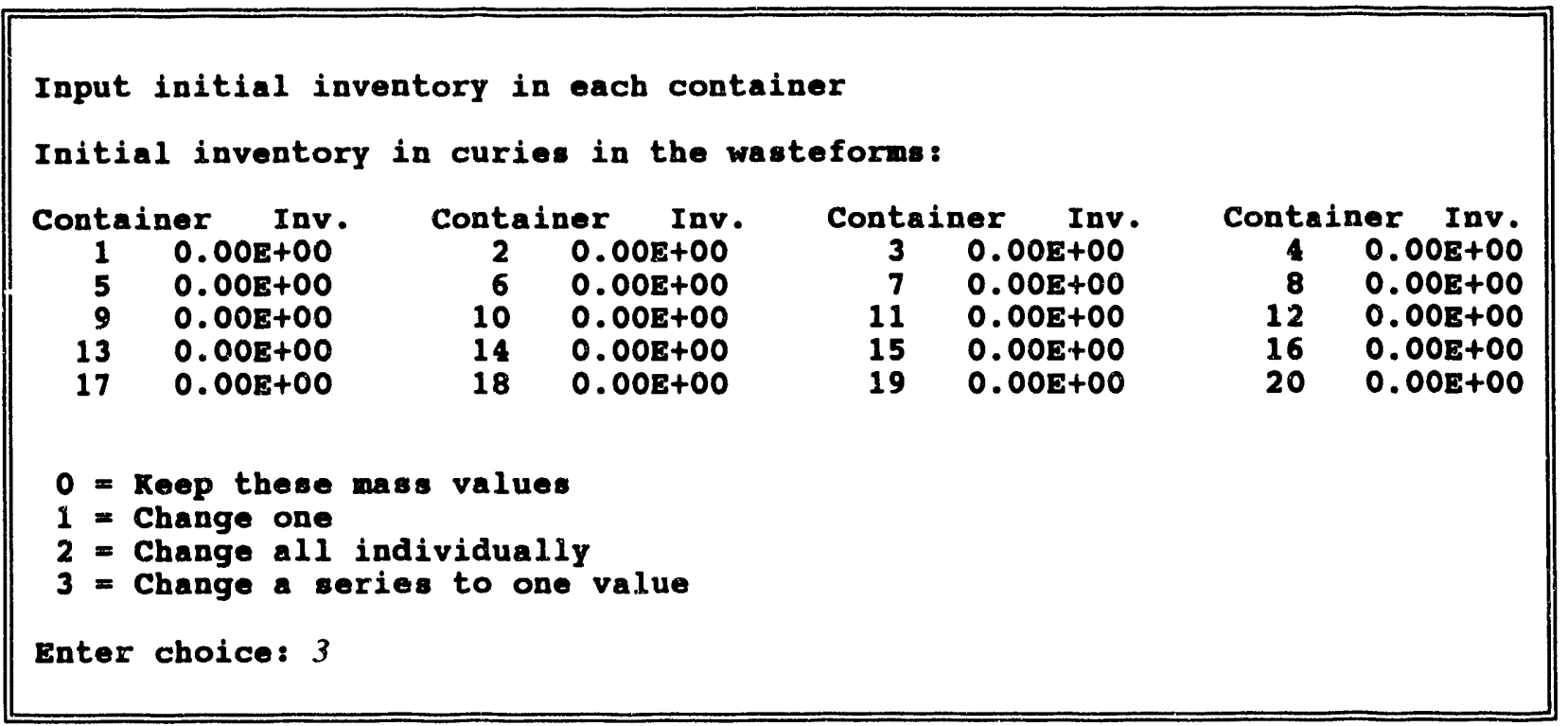

The next few lines define waste containers $1-20$ as having an initial inventory of 10 Curies. 


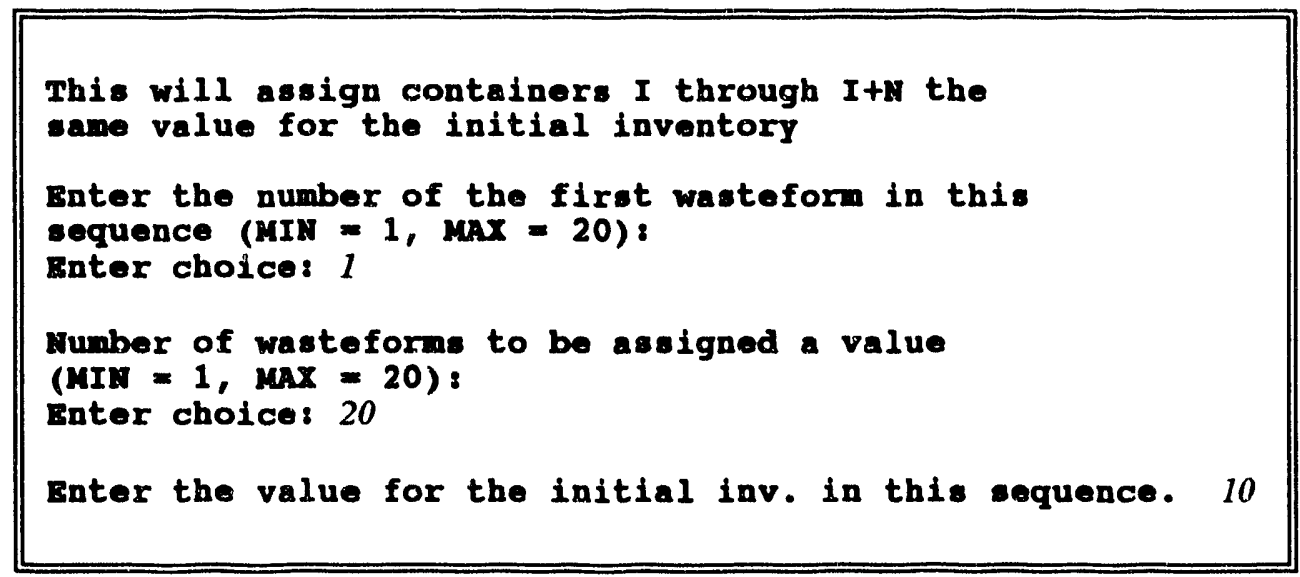

After completing the input, the current values for the inventory are printed and the user is asked if further changes are required.

\begin{tabular}{|c|c|c|c|}
\hline $\begin{array}{rc}\text { Container Inv. } \\
1 & 1.00 \mathrm{E}+01 \\
5 & 1.00 \mathrm{E}+01 \\
9 & 1.00 \mathrm{E}+01 \\
13 & 1.00 \mathrm{E}+01 \\
17 & 1.00 \mathrm{z}+01\end{array}$ & $\begin{array}{rr}\text { Container } & \text { Inv. } \\
2 & 1.00 \mathrm{E}+01 \\
6 & 1.00 \mathrm{E}+01 \\
10 & 1.00 \mathrm{E}+01 \\
14 & 1.00 \mathrm{E}+01 \\
18 & 1.00 \mathrm{E}+01\end{array}$ & $\begin{array}{cc}\text { Container Iny. } \\
3 & 1.00 \mathrm{E}+01 \\
7 & 1.00 \mathrm{E}+01 \\
11 & 1.00 \mathrm{0}+01 \\
15 & 1.00 \mathrm{0}+01 \\
19 & 1.00 \mathrm{E}+01\end{array}$ & $\begin{array}{cc}\text { Container } & \text { Inv } \\
4 & 1.00 \mathrm{E}+01 \\
8 & 1.00 \mathrm{E}+01 \\
12 & 1.00 \mathrm{E}+01 \\
16 & 1.00 \mathrm{E}+01 \\
20 & 1.00 \mathrm{E}+01\end{array}$ \\
\hline $\begin{aligned} & 0=\text { Keep these } \\
& 1=\text { Change one } \\
& 2=\text { Change all } \\
& 3=\text { Change a se } \\
& \text { Enter choice: }\end{aligned}$ & $\begin{array}{l}\text { values } \\
\text { ividually } \\
\text { to one value }\end{array}$ & & \\
\hline
\end{tabular}

\subsubsection{Waste Type Assignment to Each Container}

This variable acts as a flag that links a set of wasteform release rate parameters to a specific container and is required only if more than one set of release parameters are defined. The input routine is similar to that used for inventory assignment. The user has the option of changing a single waste type assignment, changing all waste type assignments individually, or changing a sequence of assignments to a single value.

Upon entering this menu, the code prints the current values and asks the user if further changes are required. In this example, the first 10 containers are assigned waste type 1 . 


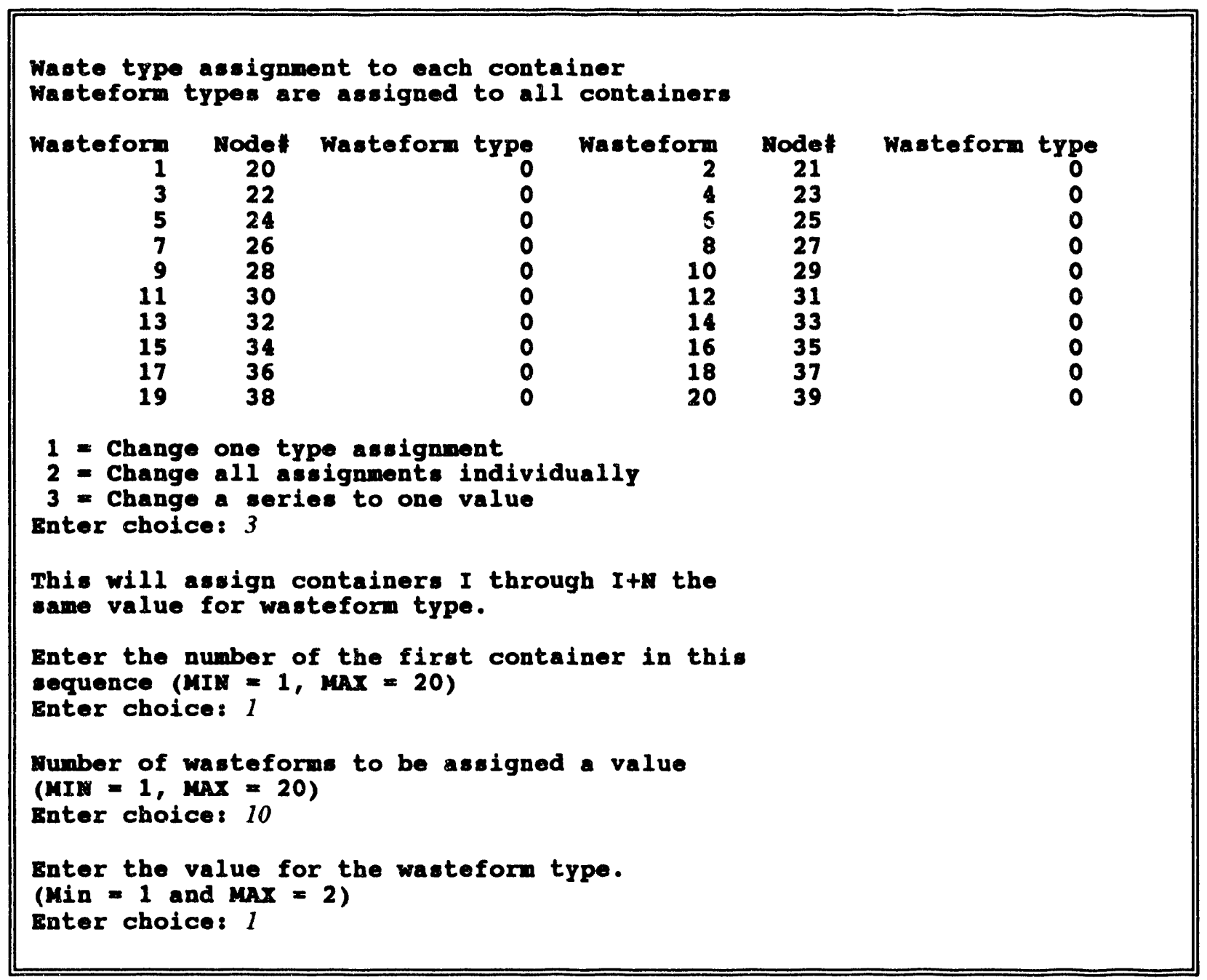

At this point, the program would print out the existing values for waste type assignment and require the user to input more values. The code requires that all waste type assignments are non-zero before allowing the user to return to the wasteform menu.

\subsection{External Source/Sink Terms}

The FD transport model permits the user to supply an external volumetric source/sink term. This term represents the rate of injection/removal of mass into the volume represented by one finite difference control volume in the system. The external source term is specified as a function of time through tabular input. Values are obtained through interpolation using the table. In the DUST code this term has units of mass $/ \mathrm{cm}^{3} / \mathrm{s}$. The mass units are determined by the activity flag, Menu 1.3. If the mass units are curies, the DUST code internally transforms this into grams to maintain consistency. The main menu for Source/Sink parameters follows. 


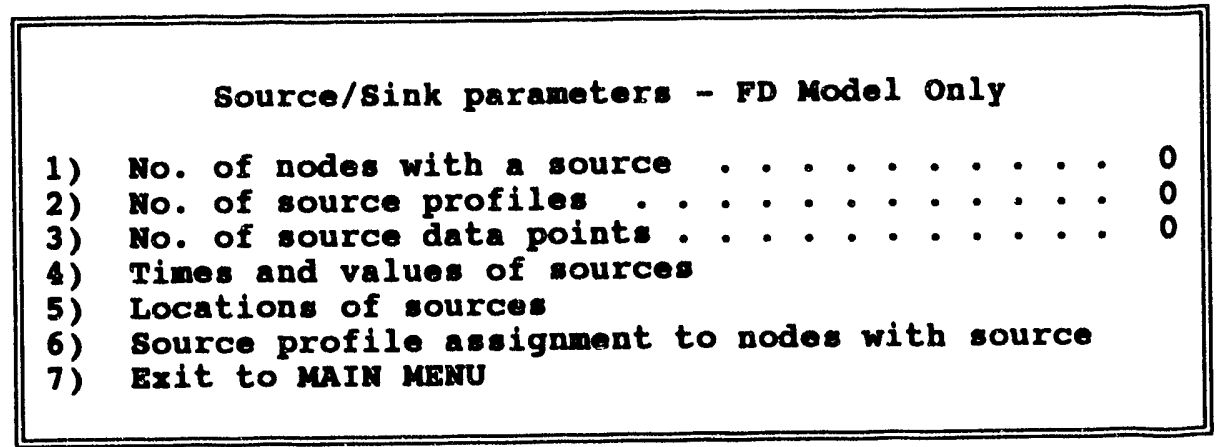

If there are no external sources, items $4-6$ o. the menu are not used by the DUST code. DUSTIN prevents the user from inputting values for these items when the number of source nodes is zero.

\subsubsection{Number of Source Nodes}

The total number of locations where a source exists ranges up to 500 , however, it is not to exceed the number of nodal (control volume) points specified in Menu 1.4.

\subsubsection{Number of Source Profiles}

The DUST code accepts up to 8 different source profiles. This value must be non-zero if the number of source nodes is non-zero.

\subsubsection{Number of Source Data Points}

Each source profile table must contain at least two points and no more than 8 points. The specified number of data points applies to every profile.

\subsubsection{Times and Values of the Sources}

The magnitude of the source is specified in a table which coniains a set of ordered pairs of time (years) and source strength (mass $/ \mathrm{cm}^{3} / \mathrm{s}$ ). The code prompts the user for the ordered pairs to be entered on a single line.

In the following example, the user previously requested 2 source profiles with 4 data points. The code prints the current values for the table and then asks if further changes are requested. In this case, profile number 1 has a concentration of $1 \mathrm{E}-5 \mathrm{Ci} / \mathrm{cm}^{3} / \mathrm{s}$ between 0 and 10 years. This decreases to zero at 10.01 years and remains there until 1000 years. Interpolation is used to determine the calculation at times not in the table. Therefore, the first time should be the problem start time (normally 0 ) and the last time should be greater than the maximum problem time, Menu 2.7. 


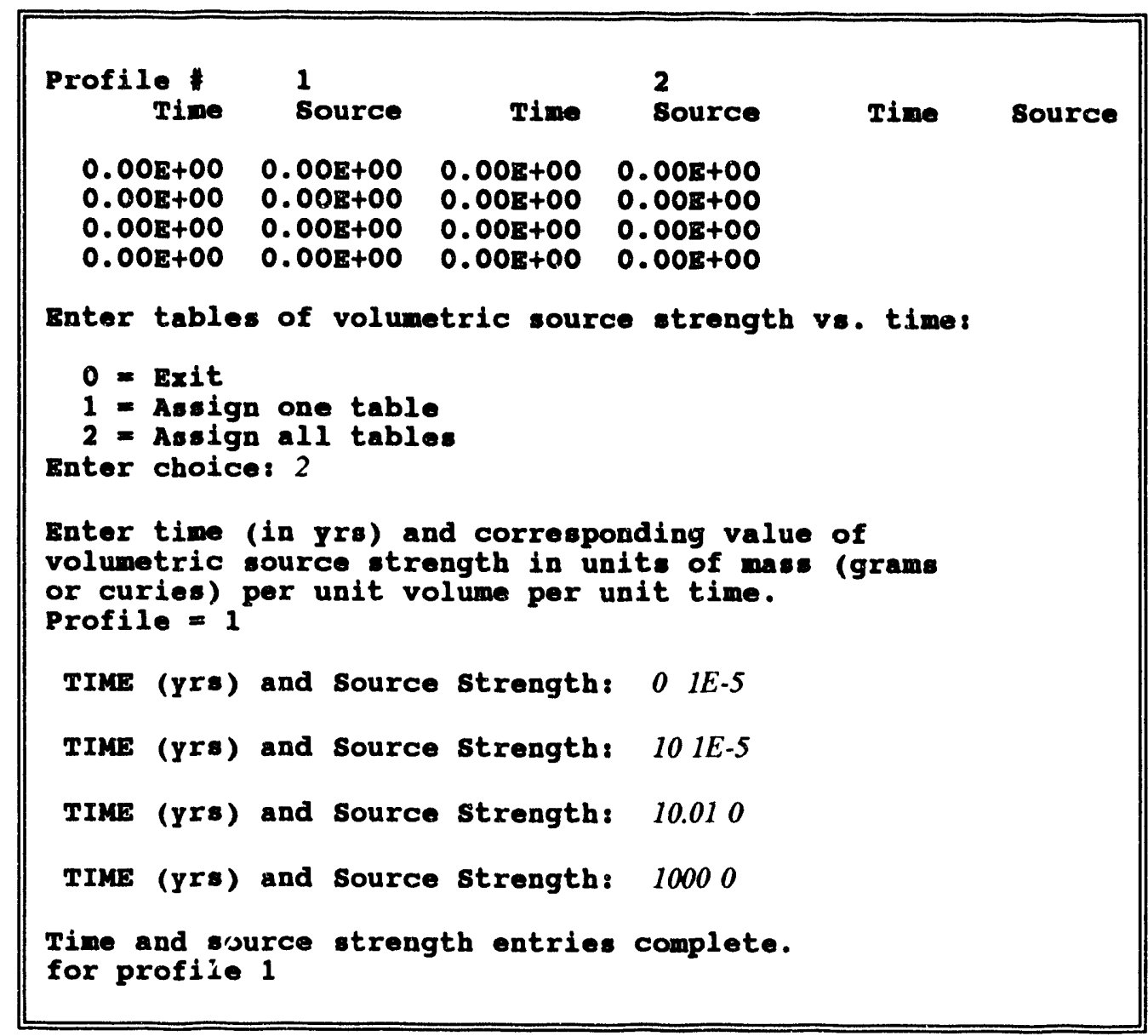

At this point, the code would prompt the user for the second source profile. Upon completion of this input, the current values are printed for review.

\subsubsection{Source Locations}

Each source must be assigned to a specific nodal location within the modeled domain. In the following example, the user requested only two source points. These are specified as occurring in Node 30 and 40. 


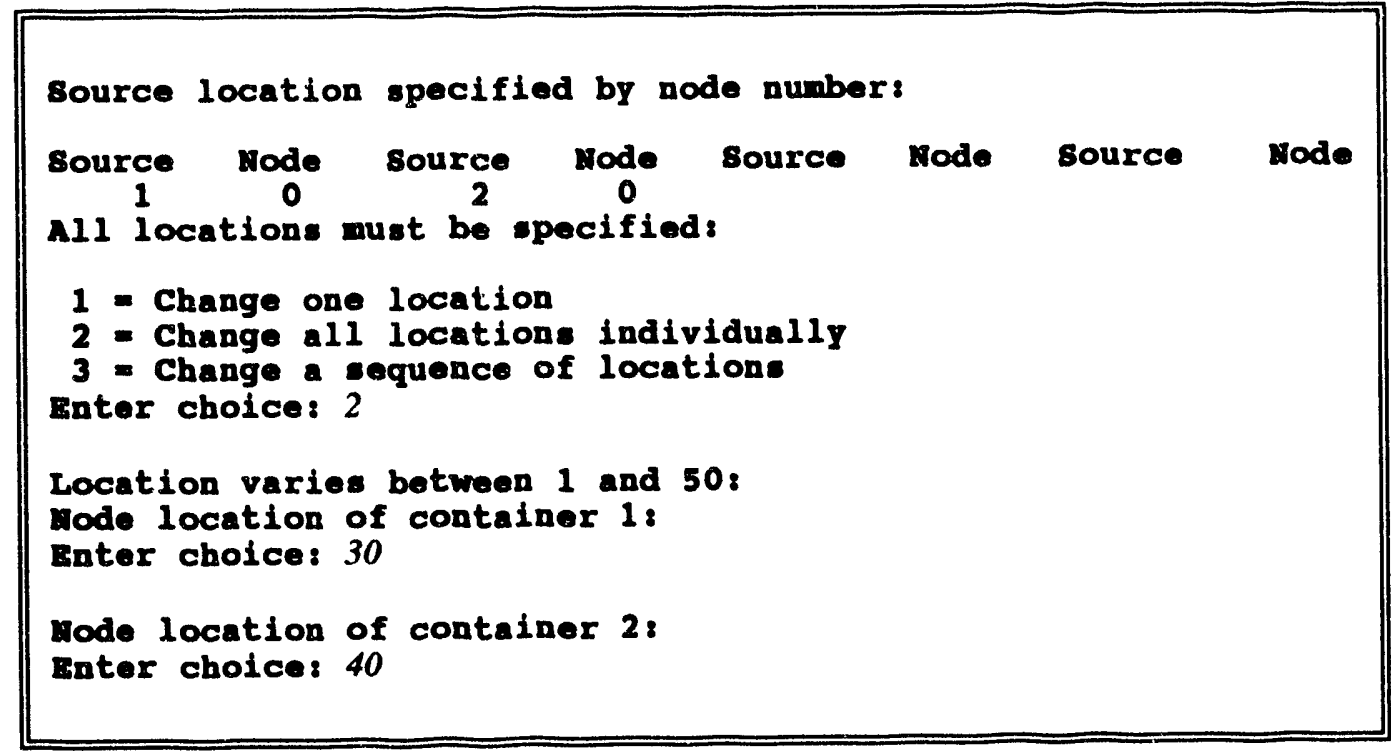

\subsubsection{Source Type Assignments}

Each of the different source nodes must be assigned a source type. In this example, there are two source nodes and two source types. Source type 1 is assigned to source 1 in node 30 . Source type 2 is assigned to source 2 .

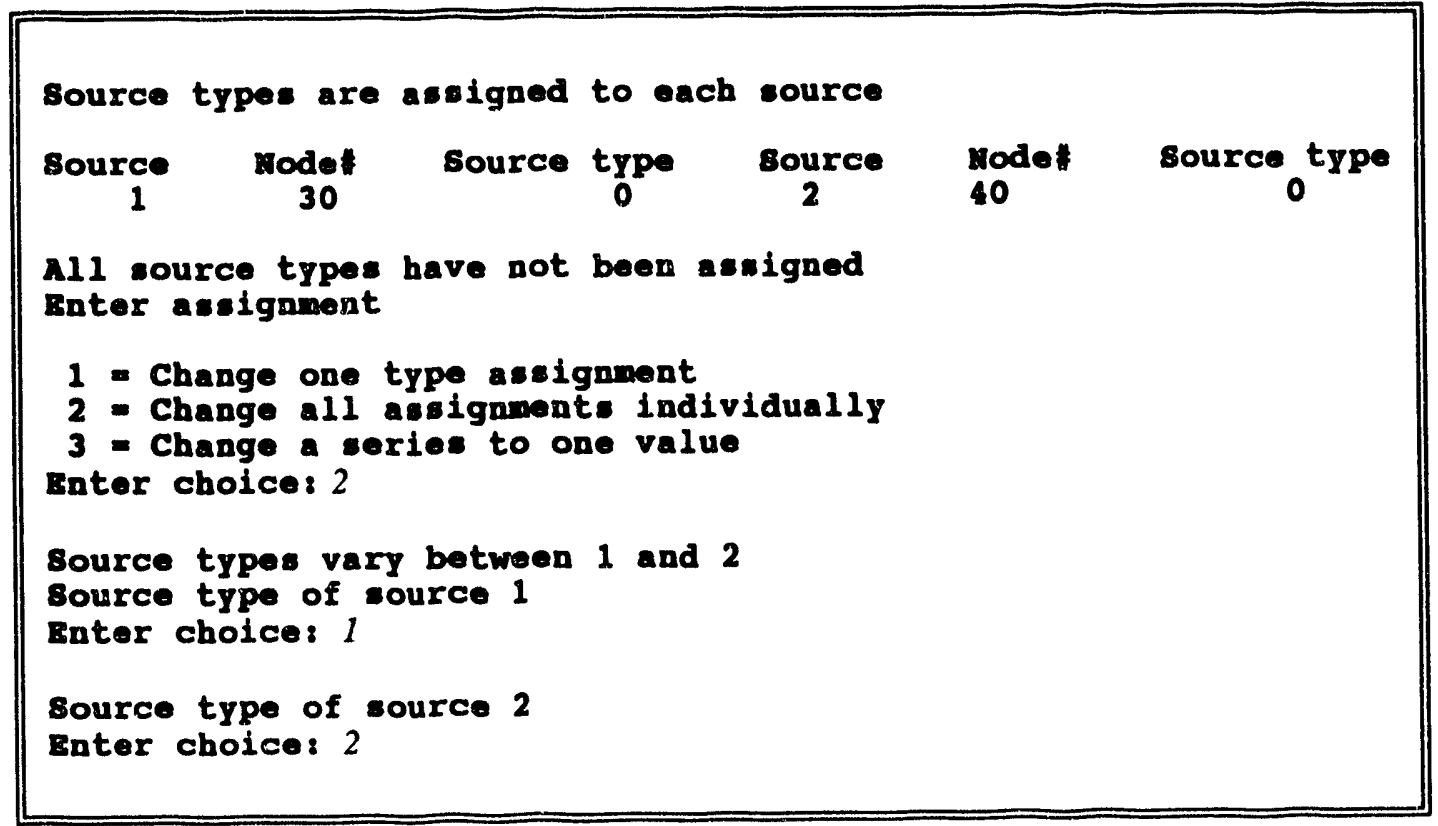




\subsection{Create an Input Deck for Use by the DUST Code}

After specifying all of the input variables, the user may attempt to create a DUST input deck. Upon entering this menu, the code prompts the user for the file name to give to the input deck. This file name may include the path. If the path is omitted, the file is stored in the current directory. The user is also asked if an attempt should be made to read the input file using the routine found in the DUST code. If this is not done, or the input deck is valid, control is returned to the main menu. If an attempt is made to read an input deck that is not valid, execution of DUSTIN is terminated and control is returned to the operating system. A typical input session follows.

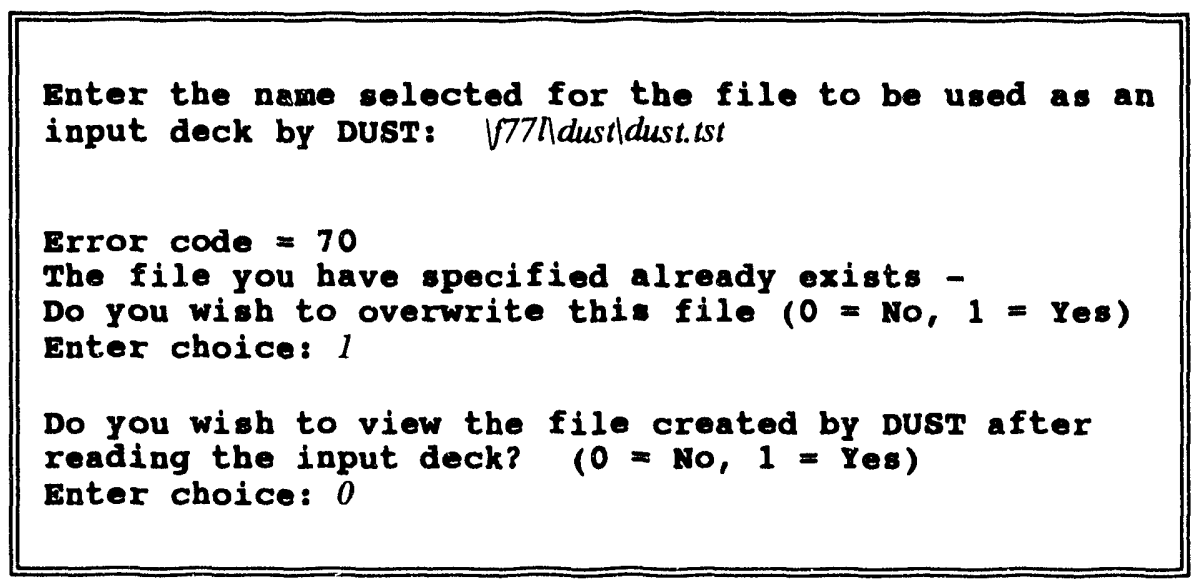

If the input file created by this process is not valid, attempts to use the DUST code will cause the program to "bomb." Also, attempts to read this file back in to DUSTIN will cause DUSTIN to "bomb" because the input routines are identical. For this reason, it is strongly recommended to create a BLOKXXX file prior to creating an input deck.

\subsection{Store Partial Input in a BLOKXXX File}

If the code user has not completed the process of creating an input deck, or if a backup copy of all specified input is desired, a BLOKXXX file may be created. All input variables required by the DUST code are part of a NAMELIST. This NAMELIST is written to the BLOKXXX file when using this routine. The XXX in the file name is a three-digit indicator that can be used to uniquely identify separate files.

A typical input routine using this menu follows. If the file exists, the code asks the user if the file is to be overwritten. 


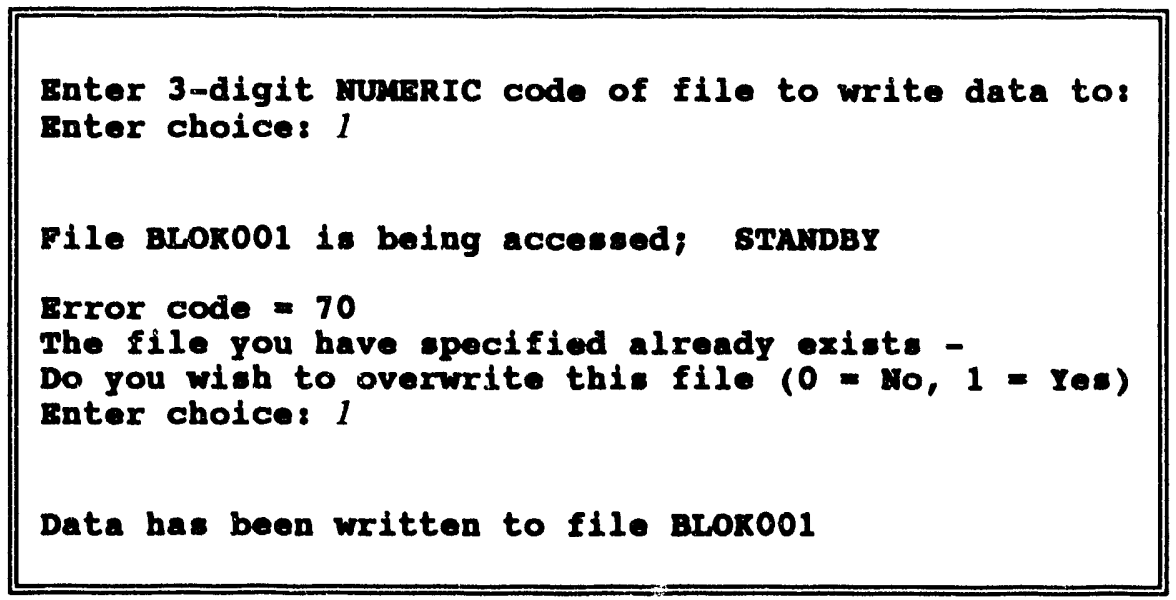

\subsection{Exit the Program}

This selection exits the program without saving data and returns control to the operating system. 


\section{STRUCTURE OF A DUST INPUT DECK}

Chapter 6 provides a detailed description of the procedure used when creating an input deck for the DUST code using the pre-processor DUSTIN. Although DUSTIN permits the modification of any single variable used by the DUST code, often times, when performing multiple runs that it would be quicker and easier to change the input file directly. For this reason, a complete description of the structure of the input decks is provided. There are substantial differences between the input required for the FD and MCMC transport models. Therefore, caution must be used when modifying the input decks directly to switch between these models.

A DUST input deck is composed of a series of Data Sets which are groupings of data covering a particular topic. There are 10 Data Sets. These Data Sets correspond to the first 10 menu items on the main menu in the code DUSTIN and are:

1) General problem definition;

2) Time parameters;

3) Material assignments/properties;

4) OUTPUT specifications;

5) Facility dimensions and coordinates;

6) Initial and boundary conditions;

7) Water flow and moisture content;

8) Container parameters;

9) Wasteform parameters; and

10) Source/Sink parameters (FD Model Only).

The first nine Data Sets are required for every problem, however, the content of the Data Sets varies depending on the choice of transport model (FD or MCMC) and the problem. For example, if wasteforms are not modeled, Data Set 8 would contain one data input card telling the code that the number of containers is zero and Data Set 9 would contain a similar card indicating that there are no wasteforms. Data Set 10 is used only when the finite difference transport model is used.

Within a DUST input deck, there are two major categories of input cards, label cards and data input cards (for notational convenience, each line of the input deck is referred to as a card). The beginning of each Data Set within DUST is denoted with a label card. If the input deck was created using DUSTIN, this card will contain the name of the Data Set and a brief description of its contents, e.g., "Data Set 8 - Container Parameters." If the code user creates the deck without DUSTIN or modifies the DUSTIN deck, the label can be any alpha-numeric string with a length of 79 characters or less. For example, the user may want to put more information on the identifier, e.g., "DS-8 - Container Parameters - Multiple Failure Times," would be an acceptable label. Following the Data Set label card are the data input cards. In the data input cards, the first 10 columns on each card is reserved for comments supplied by DUSTIN or the code user. The comments are used to identify the variables contained on the card and can be 
any alpha-numeric string of up to 10 characters. For example, for the container parameter TIMEB, which gives the time of general failure of the container, the comment included by DUSTIN is "TIME FAIL." As with the label cards, DUSTIN provides a comment for each data input card. DUSTIN writes a blank label card to indicate the end of the data set.

Label cards are also used to identify the major property input parameters which are input several to a card. These include parameters describing transport in soil, container degradation, and wasteform release. For example, as many as seven wasteform release parameters will appear on a single card. DUSTIN creates a label card that identifies each of these parameters. These label cards will be discussed in the appropriate sections of this chapter.

A typical finite difference input deck is displayed in Table 7.1. The counterpart for the MCMC model is in Table 7.2. The input deck follows the order displayed in the main menu of the pre-processor DUSTIN. These two input decks model the same problem. Any differences that arise are due to the different data requirements of the transport models.

The modeled problem has 106 mixing cells or 112 control volumes. The additional control volumes are needed to move the source away from the boundary. There is only one material type. There are 53 containers, the first 20 fail after 5 years, the remainder after 6 years. There are two wasteform types, both release half the mass via the rinse mechanism and the other half through uniform release. The fractional release rate varies between the two wasteform types. The first 20 wasteforms are type 1, the remainder are type 2. A plot of concentration versus time at the two trace locations is given in Figure 7.1.

The remainder of this chapter provides the structure of the DUST input deck. All input is read into the code through formatted read statements. For clarity, each of the formatted read statements will be supplied along with the FORMAT statement. These are supplied in the single line boxes in this chapter. Often at the top of the box, a few lines in italics will appear. These are the actual input lines found in Tables 7.1 and 7.2. For general input, Table 7.1 will be used in the examples. If the input is specific to the MCMC transport model, data from Table 7.2 will be used. A blank line is often used to signal the end of a data set. These are indicated in the examples of this chapter by the words "BLANK LINE."

The code DUST makes extensive use of the subroutines INPTR, INPTI, INPTR2, READR, and READN for reading the input data. These procedures are explained below and will be referred to later in this chapter as necessary. 
Table 7.1 Sample finite difference input deck for a single material with containers failing at either 5 or 6 years. Release mechanisms include rinse and uniform dissolution. Blank cards are denoted by the word BLANK in columns $1-5$.

FDM EXAMPLE INPUT DECK

$\begin{array}{lllll}\text { RN Prop CS-137 } 3.017 E+01 & 1.000 E+01 & 1.370 E+02 & 1\end{array}$

NNP ITRANS $112 \quad 1$

BLANK

NTI DTCHG 1000 DATA SET 2: Time paraneters

TIME STEP 4.010E+00 1.000E-02 1.000E-02 1.600E+01

TIME DTCHG $1.600 \mathrm{E}+01$

BLANK

MMAT NCM

DATA SET 3: Material parameters
$\mathbf{K}-\mathbf{d}$ DENSITY DISP. DIFFUSION
0

BLANK

DATA SET 4: Output Control parameters

Print Control Parameters for each time step (NTI)

2222222222222222222222222000000000000000000000000000000000000000000000 0000000000000000000000000000000000000000000000000000000000000000000000 0000000000000000000000000000000000000000000000000000000000000000000000 0000000000000000000000000000000000000000000000000000000000000000000000 0000000000000000000000000000000000000000000000000000000000000000000000 0000000000000000000000000000000000000000000000000000000000000000000000 0000000000000000000000000000000000000000000000000000000000000000000000 0000000000000000000000000000000000000000000000000000000000000000000000 0000000000000000000000000000000000000000000000000000000000000000000000 0000000000000000000000000000000000000000000000000000000000000000000000 0000000000000000000000000000000000000000000000000000000000000000000000 0000000000000000000000000000000000000000000000000000000000000000000000 0000000000000000000000000000000000000000000000000000000000000000000000 J000000000000000000000000000000000000000000000000000000000000000000000

Trace var 00000000000000000000

Con Tr LOC $\quad 32 \quad 61 \quad 82 \quad 111$

$\begin{array}{lllll}\text { FX TI LOC } & 32 & 61 & 82 & 111\end{array}$

BLANK

Hght Area Delta-x

BLANK

BLANK

INIT INV

BLANK

BC FLAGS

TIME

VALUE

TIME

VALUE

BLANK

DATA SET 5: Facility Coordinate Data

$1.110 \mathrm{E}+02 \quad 6.500 \mathrm{E}+03$

$$
\begin{array}{cccccc}
1 & 112 & 1 & 0.000 E+00 & 1.000 E+00 & 0.000 E+00
\end{array}
$$

$$
\begin{array}{ccc}
\text { DATA SET } & 6: \text { Inttial and Boundary Conditions } \\
1112 & 1 & 0.000 \mathrm{E}+000.000 \mathrm{E}+00
\end{array}
$$$$
\begin{array}{lll}
1 & 1 & 2
\end{array}
$$

$0.000 \mathrm{E}+00 \quad 1.600 \mathrm{E}+01$

$0.000 \mathrm{E}+00 \quad 0.000 \mathrm{E}+00$

$0.000 \mathrm{E}+001.600 \mathrm{E}+01$

$0.000 \mathrm{E}+00 \quad 0.000 \mathrm{E}+00$ 


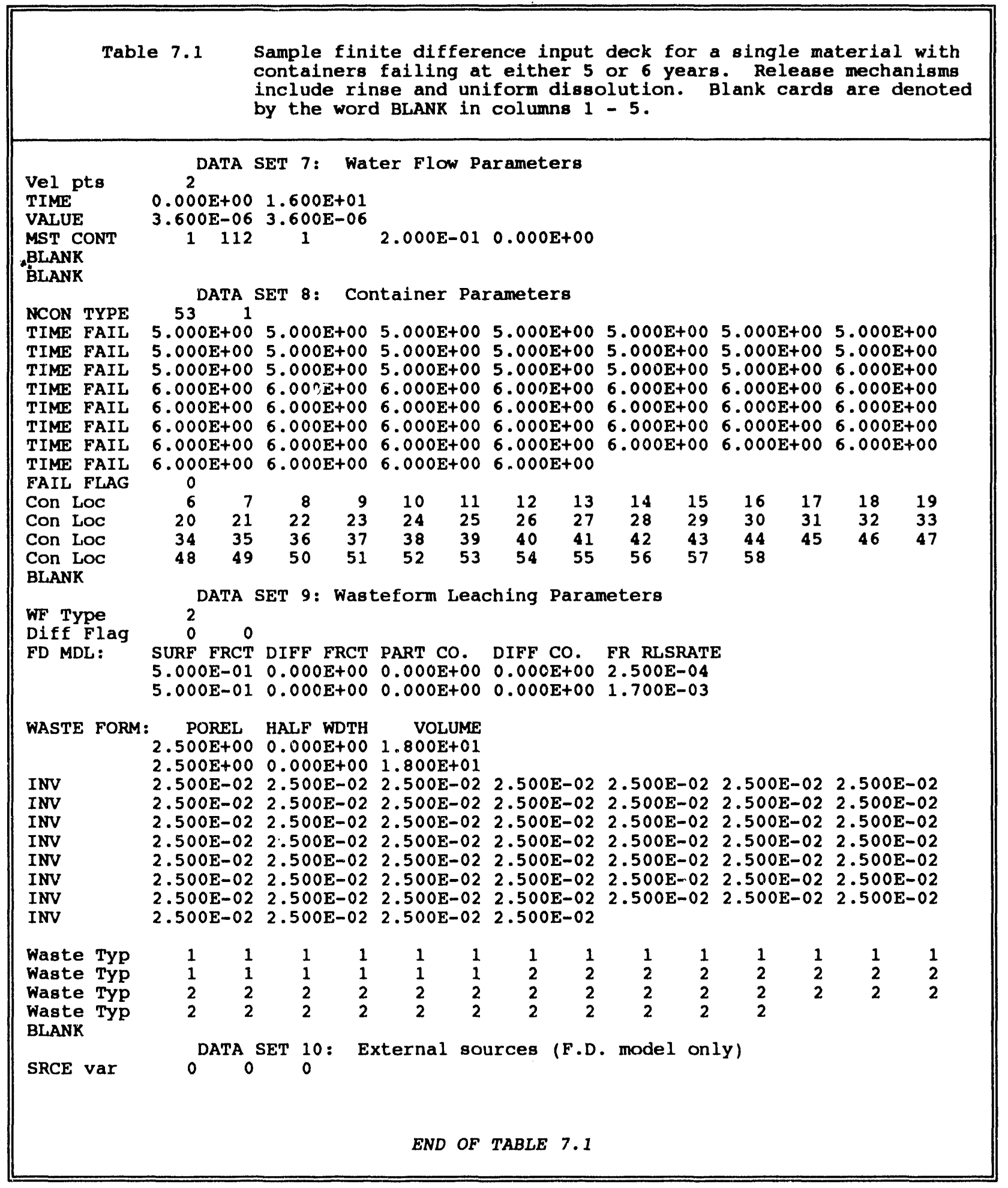




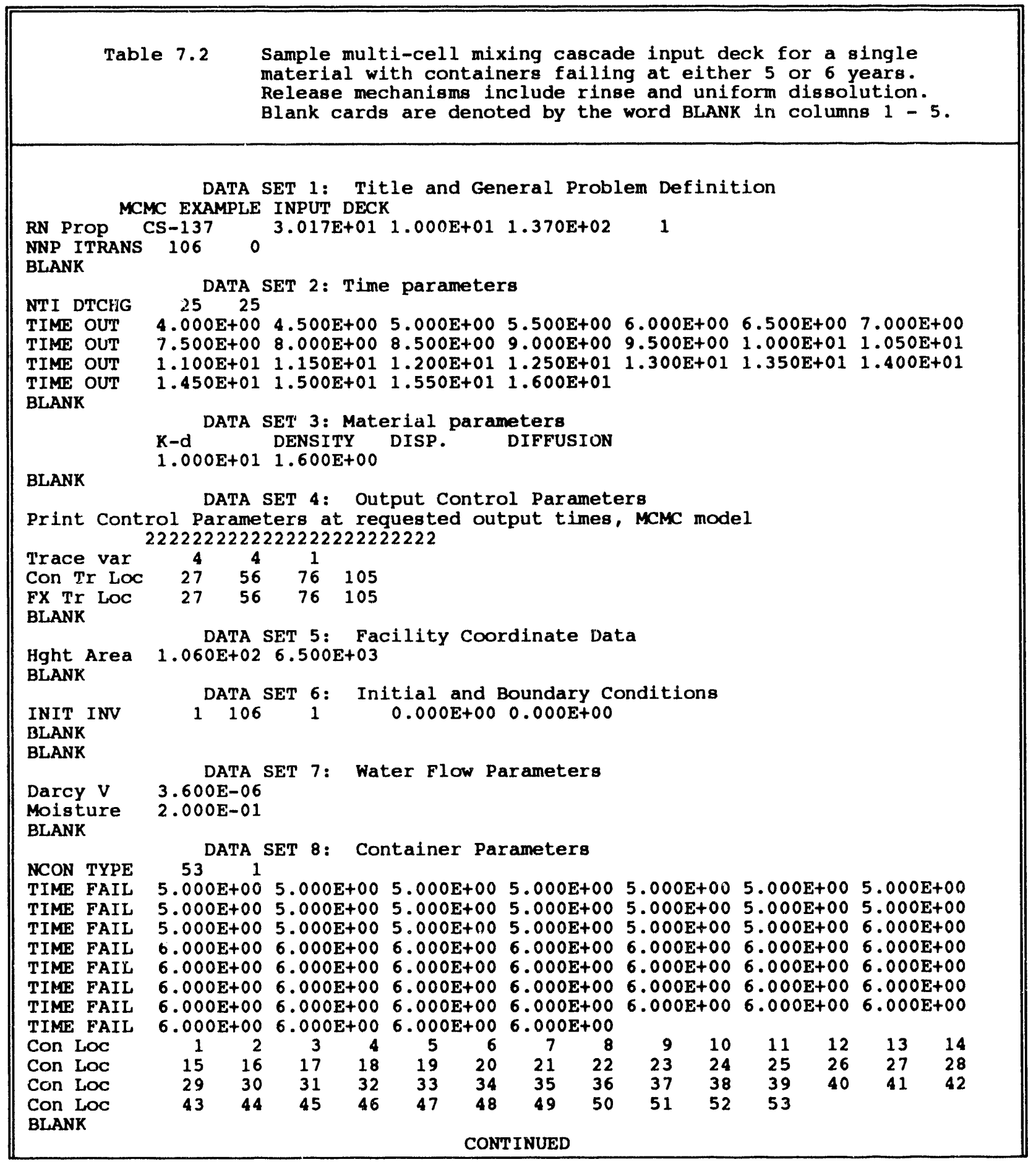




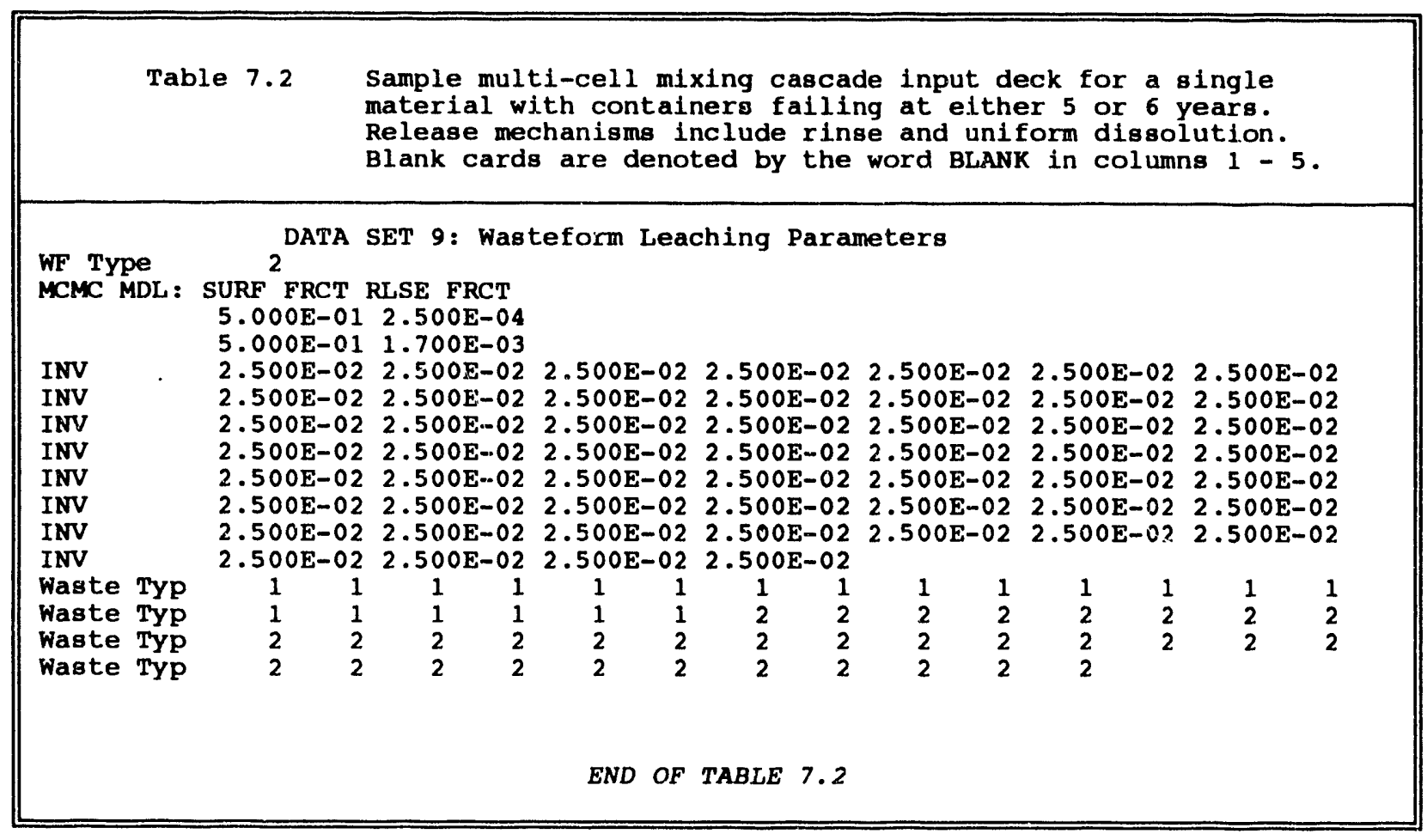




\section{COMPARISON OF MIXING CELL MODEL AND FINITE DIFF. MODEL}

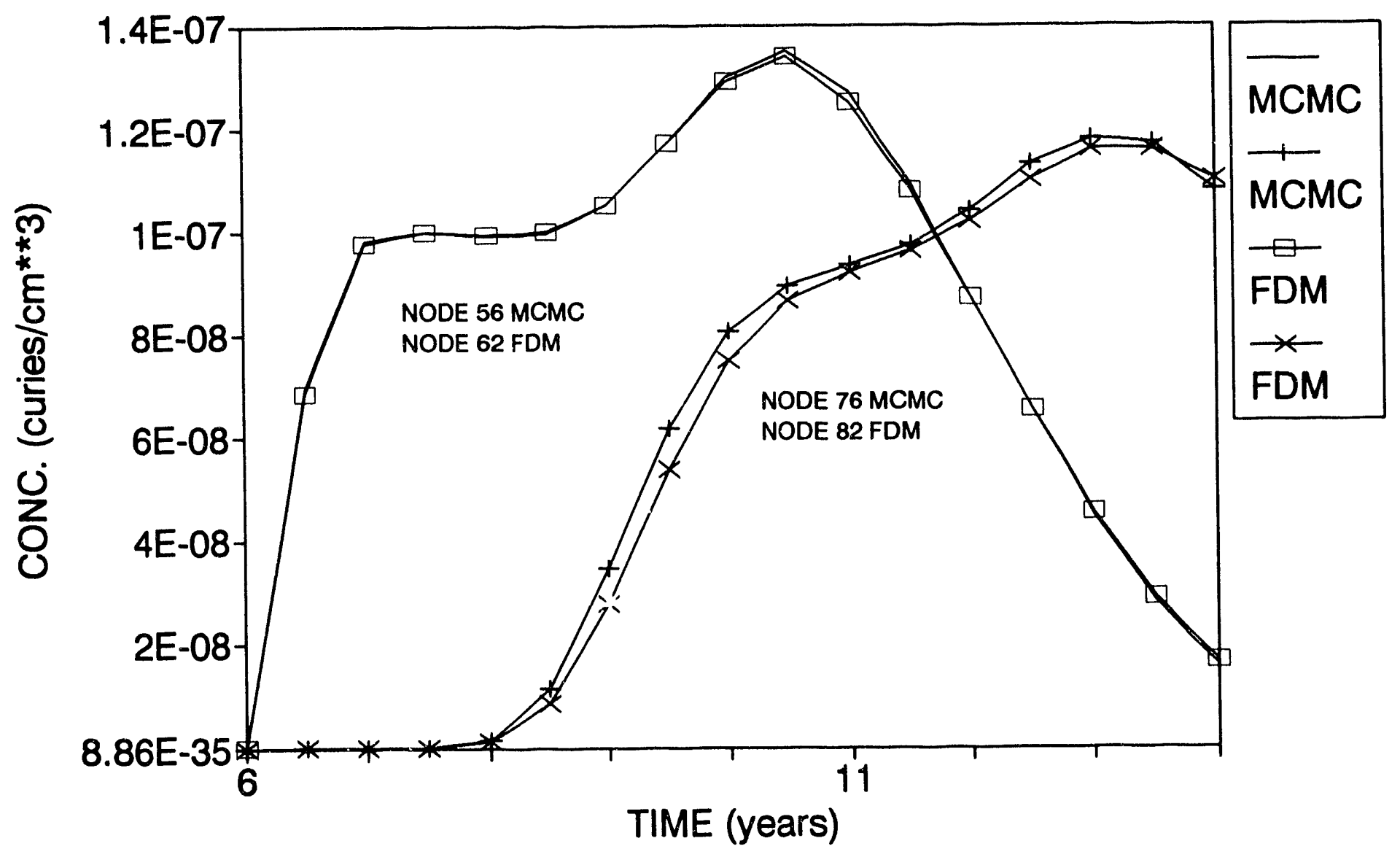

Figure 7.1 Plot of concentration versus time 3 and $23 \mathrm{~cm}$ beneath the last wasteform for the test problems in Table 7.1 (FD) and Table 7.2 (MCMC). 


\section{Subroutine INPTR}

The DUST code contains a subroutine, INPTR, which reads in real variables in blocks of 7 with a label in columns 1 - 10. The formatted read statement in INPTR is:

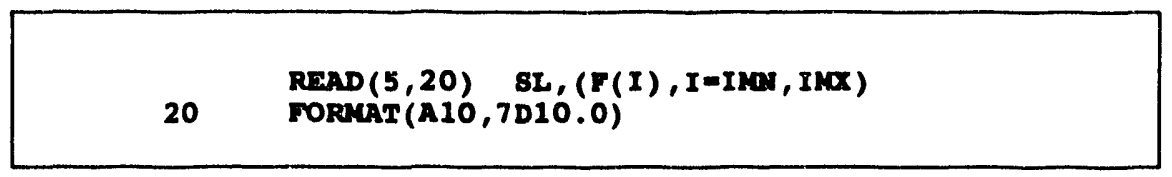

The variables, IMN and IMX are calculated by the code. In INPTR, there is a DO WHILE loop which increases the value of IMN until it exceeds the maximum number of input values requested. For example, the first time through the loop, IMN $=1$ and $I M X=7$. The second time through the loop, IMN $=8$ and $\mathrm{IMX}=14$, and so on. The value for IMX is limited to be no greater than the maximum number of variables to be specified. For example, if the maximum number to be specified is 10 , the second time through the loop IMX $=10$. In attempting to go through the loop a third time, IMN would be 15 which is greater than the maximum. Therefore, the input routine is terminated.

The array $\mathrm{F}$ and the maximum number of variables to be specified are determined through arguments in the call statement.

Subroutine INPTR is used to define the variables TIMOUT (output times for the MCMC model, times at which the time step is reset in the FD model), TIMEF (time of container failure), and WTINIT (initial mass in each container).

\section{Subroutine INPTI}

Subroutine INPTI is the analogue of INPTR for integer numbers. In this subroutine, 14 integer values are input per card. The read statement is:

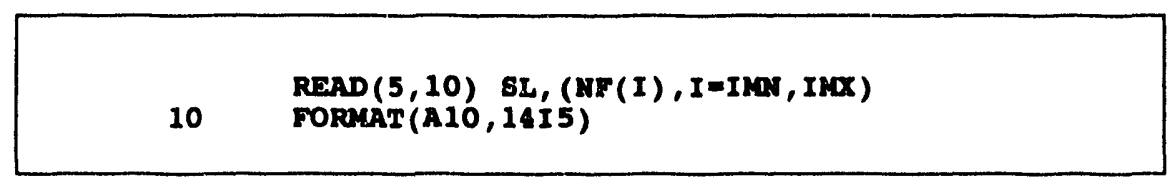

The first time through, IMN is 1 and IMX is the minimum of 14 or the maximum number to be redefined. Again, these values are increased in steps of 14 until IMN exceeds the maximum value.

The array NF and the maximum number of input variables are defined through calling arguments. 
Subroutine INPTI is used to define NTRCEC (nodes for concentration traces), NTRCEF (nodes for flux traces), IPIT (flag for modeling local failure), NELCON (nodal location of all containers), ICTYPE (container type at each location), IDIFF (geometry flag for the diffusion model), IWTYPE (waste type at each location), LSRC (nodal location of external sources), and ISTYPE (source type at each location).

\section{Subroutine INPTR2}

Subroutine INPTR2 is identical to INPTR except that instead of defining a onedimensional array, a two-dimensional array is defined. Two dimensional arrays are used to specify all of the tables involving a variable as a function of time. The read statement is:

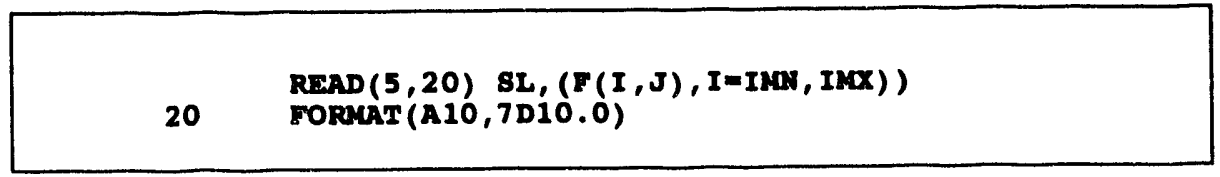

The procedure used to define IMN and IMX is identical to that used in INPTR. The dummy array $F$, the maximum number of values to be defined, and the index $J$ are all defined in the calling arguments. Typically, the call statement for INPTR2 is embedded in a loop in which $\mathrm{J}$ increases from 1 to the number of profiles requiring input.

Subroutine INPTR2 is used to define the tables of boundary condition versus time at both boundaries, Darcy velocity versus time, and source strength versus time.

\section{Subroutine READR}

READR takes a set of six numeric values and calculates a series of coordinates based on these values. These values are:

1) Number of the first node in the sequence, NI

2) Number of nodes defined by the sequence, NSEQ

3) Number to add to each node in the sequence, NAD

4) Value for the first node in the sequence, FNI

5) Value to add to each node in the sequence, FAD

6) Fractional increment in value for each node in the sequence, FRD

The location is specified through the following equation:

$$
\mathrm{F}\left(\mathrm{NI}+\mathrm{I}^{*} \mathrm{NAD}\right)=\mathrm{FNI}+\mathrm{FAD}^{*}(1+\mathrm{FRD})^{* *}(\mathrm{I})
$$


In the example presented in Table 7.1, the user specified the location of all 112 nodes to be $1 \mathrm{~cm}$ apart. With the following card:

\section{$\begin{array}{llllllll}\text { Delta-X } & 1 & 112 & 1 & 0.000 \mathrm{E}+00 & 1.000 \mathrm{E}+00 & 0.000 \mathrm{E}+0\end{array}$}

The format for this input statement in subroutine READR is:

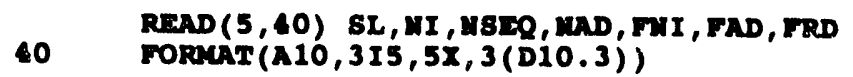

The subroutine READR continues to read input until the value for NI is zero. Therefore, a blank card is often used to signify the end of all READR input variables. After a zero is input, the code checks to insure that all NNP locations are defined (NNP is the number of nodal points specified in Menu 1). READR is also used for input of initial concentration and moisture content at each location.

\section{Subroutine READN}

READN is only used when redefining the location at which certain material properties exist. For this reason, the discussion of READN can be found in Section 7.3.

\subsection{DATA SET 1: Title and General Problem Definition (Menu 1)}

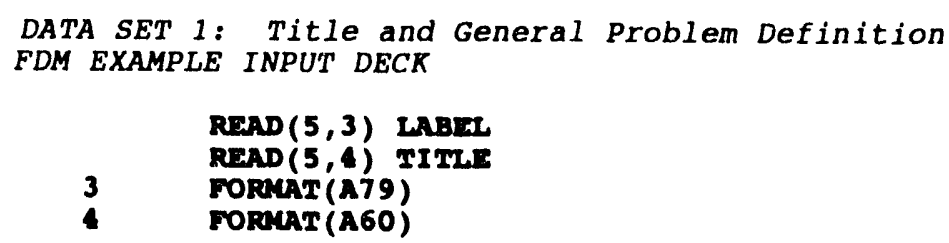

The first card in the input deck is the label for Data Set 1. The second card contains the title for this problem and can be up to 60 characters in length. Both the MCMC and the FD models require these cards. The format for reading the LABEL remains unchanged in DUST and will not be repeated in the remainder of this chapter.

Both the MCMC and FD models require the two data input cards in this section. The first card contains the alpha-numeric name for the radionuclide, the half-life in years, the atomic mass, the solubility limit in $\mathrm{gm} / \mathrm{cm}^{3}$, and the mass activity flag. The second card contains integer 
parameters to define the number of computational points and the flag for selecting the transport model. The READ statements are:

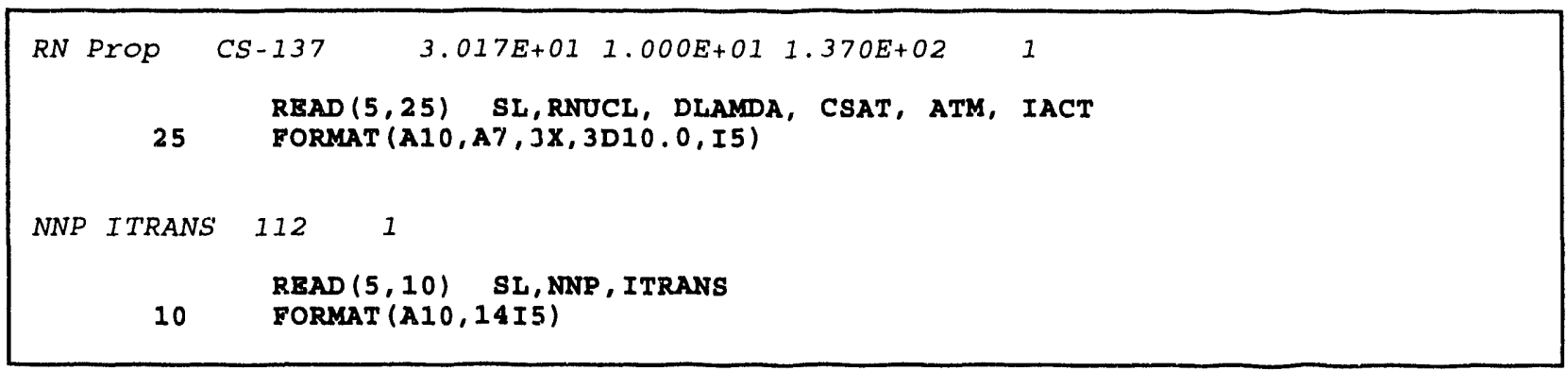

The parameter SL appears at the beginning of each data input card and is the 10 character label used to identify the data on the card. The FORMAT identified by statement 10 is used to read in all integer parameters used within DIST. The variables LABEL and SL are not used by the DUST code. Their sole purpose is to identify variables in the input deck.

\subsection{DATA SET 2: Time Parameters (Menu 2)}

After the two label cards, both the MCMC and FD models require the first data input card in this data set. This card contains the maximum number of time steps used when the FD transport model is used (this variable is unused in the MCMC model) and the variable NDTCHG. NDTCHG is a dual purpose variable, for the FD model it represents the number of times at which the time step is to be reset to its original value, while for the MCMC model it is the number of times at which a calculation is requested.

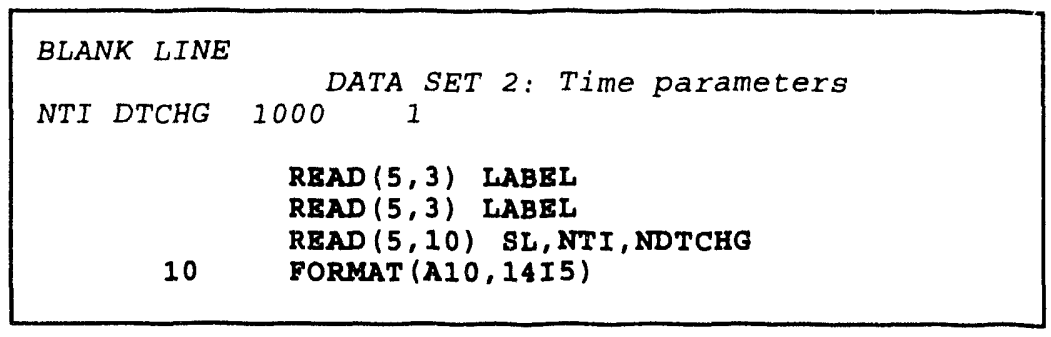

If the FD transport model is requested (ITRANS $=1$ ), the following input card which specifies the time step parameters, (initial time step, fractional change in time step, maximum time step, and maximum simulation time) is required. Inclusion of this card when the MCMC model is specified will cause an error. 
TIME STEP 4.010E+00 1.000E-02 1.000E-02 1.600E+01

20

In the example problem for the FD model, Table 7.1, the initial time step is 4.01 years while the maximum time step is $\mathbf{0 . 0 1}$ years. This is useful for taking a large time step prior to release of any contaminants. In the example, the containers do not breach until 5.0 years. However, if the user specified 5.01 years for the original time step, the code would model the breach to occur at 0 years, that is, the time of breach is defined as the time at the beginning of the time step in which the container fails. This type of approach should only be used if the boundary conditions are zero concentration or zero advective flux and the initial conditions are zero concentration. For non-zero initial or boundary conditions, the large time step may lead to large errors in the numerical solution.

The final set of data cards in this set specifies a dual purpose variable, TIMOUT. TIMOUT is the times at which the time step is reset if the FD model is used. It is the times at which a computation is performed for the MCMC model. The number of times required is specified by the variable NDTCHG. If NDTCHG $=0$ this card is not required. DUST uses the input routine INPTR to read in the values. The formatted read is:

TIME DTCHG $1.600 E+01$

20

$\operatorname{Rad}(5,20) 8 I,(I(I), I=I M a, I M x)$

romeat (A10,7D10.0)

On this call to INPTR, the dummy array $F$ represents the array TIMOUT. As on all calls to INPTR, an input card is required for every 7 values of this variable.

\subsection{DATA SET 3: Material Assignments/Properties (Menu 3)}

As with each new Data Set, the first two cards are label cards indicating the end of the previous data set and the beginning of a new data set. The input statements are:

BLANK LINE

DATA SET 3: Material parameters

READ (5, 3) IABEL

$\operatorname{RHAD}(5,3)$ IABEL 
The MCMC model permits only one material type and two material properties. In contrast, the FD model allows up to 10 material types and four material properties. Therefore, the input for the FD model is substantially greater than for the MCMC model. For this reason, the input requirements of the models are discussed separately.

\section{MCMC Model}

The MCMC model permits only 1 material (NMAT) and two material properties (NMPPM). The variable PROP $(1,1)$ is the distribution coefficient and the variable $\operatorname{PROP}(2,1)$ is the bulk density. For the material properties, an additional label card is required (therefore, there are three label cards in succession in this situation). If the DUSTIN code is used the label contains designators for these two properties in columns $11-20$ and $21-30$, respectively. The input statements are:

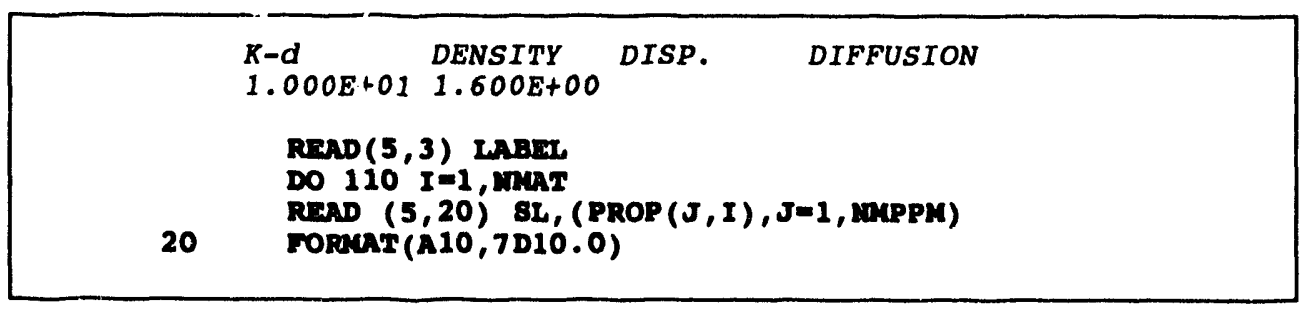

FD Model

The first card required by the FD model defines the number of materials (NMAT) and the number of materials that will be redefined from material 1 (NCM). Initially all nodes are assumed to have material properties specified by material 1 , the default material.

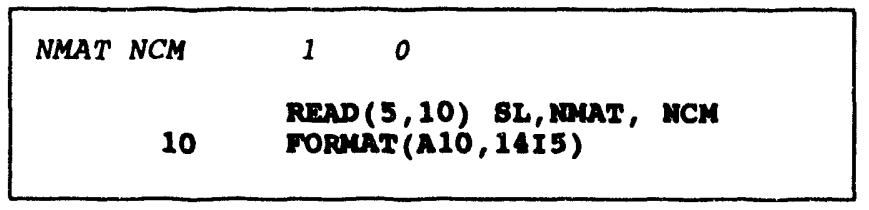

The next group of cards in this set requires the material properties for each material. The FD model has NMPPM properties (NMPPM $=4$ ). The properties for material $\mathrm{J}$ are PROP $(1, \mathrm{~J})$, the distribution coefficient; PROP(2,J), the soil bulk density; PROP(3,J), the dispersivity; and PROP(4,J), the effective diffusion coefficient (tortuosity effects should be included in this parameter). This set of cards requires a label card, similar to that needed by the MCMC model. The label card, if written by DUSTIN, contains identifiers for the four material properties. The input routine requires all properties for a material to appear on a single card. The input procedure is repeated for each material as follows: 


\begin{tabular}{|c|c|}
\hline 20 & 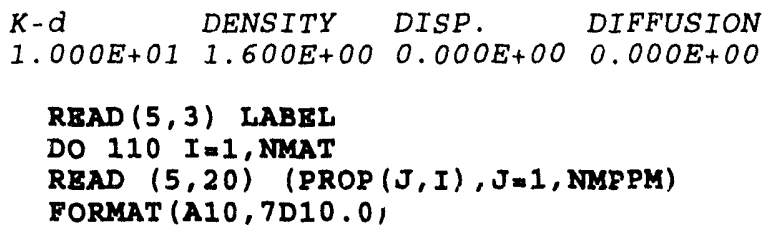 \\
\hline
\end{tabular}

In Table 7.1, the values for the dispersion and diffusion term are zero. This was done to match the MCMC model which does have these variables.

The final set of cards define the link between nodes and material type. Material type assignment is achieved using the subroutine READN. If the number of changed materials is zero, or the number of material types is 1 , this set of cards is not required, as in Table 7.1.

READN reads a sequence of cards having five integer values per card. The five values are used to automatically generate the material type reassignment. For material type assigniment, the five variables on each card are:

a) the location (node number) of the first node in the sequence (NI),

b) the number of nodes to be assigned (NSEQ),

c) the increment of nodes in the sequence (NADD),

d) the material type for the first node in the sequence (NITYP), and

e) the increment of material type for each node in the sequence (NTYPAD).

READN uses the following equation to assign material types

$$
\operatorname{IMAT}(\mathrm{NI}+\mathrm{NADD} * \mathrm{I})=\mathrm{NITYP}+\mathrm{NTYPAD} * \mathrm{I}
$$

where I ranges from 0 to NSEQ-1. The read statement is:

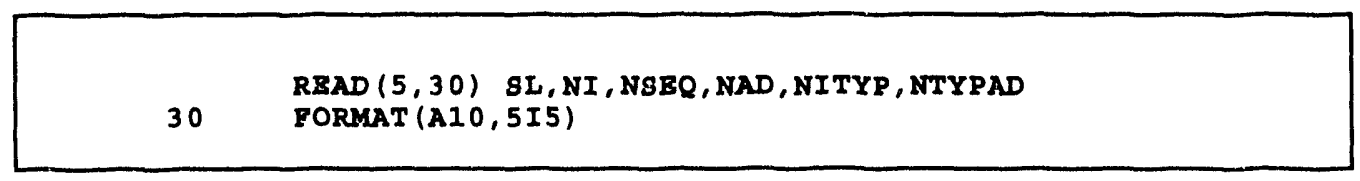

The number of cards required to redefine material properties is not fixed. READN keeps reading cards unti! NI is zero: This is often accomplished by including a blank card. Therefore if READN is used, two blank cards appear at the end of this Data Set, one to indicate the end of information for READN and one to indicate the end of the Data Set. After reading all the cards prior to the blank card, the DUST code determines if the number of material types assigned equals the number requested in variable NCM. If there is a discrepancy, an error message is printed and the code is stopped. A check is also performed to insure that the assigned material types are between 1 and the number of materials. 
An example of redefining nodes $90-99$ as material type 3 follows.

\section{NI NSEQ NADD NITYP NTYPAD \\ $\begin{array}{lllll}90 & 10 & 1 & 3 & 0\end{array}$}

For more details see Menu 3.2 in Chapter 6.

\subsection{DATA SET 4: Output Specifications (Menu 4)}

This data set begins with 3 label cards, (end of previous Data Set, beginning of this Data set, and a label for the output print flags). The next set of cards define the output requested at each computation time. This flag has up to four values, $(0=$ print nothing, $1=$ print concentrations; 2 = print 1 plus flux; and $3=$ print 2 plus wasteform release data (FD model only). The input procedure is:

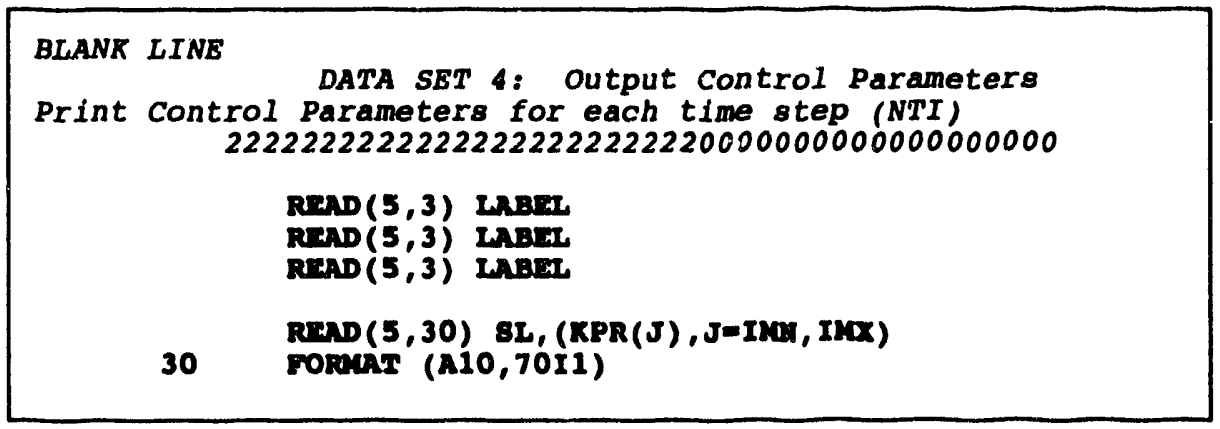

The procedure for determining KPR is identical to that used in INPTI except the format is different. The variables $\mathrm{IMN}$ and IMX start as 1 and 70, respectively, and are continually redefined until IMX equals the number of computation time intervals (NTI).

The next set of variables specify the number of concentration and flux traces as well as the number of time steps between writing these variables to their respective output files. Concentration trace variables are written to TRACECND.DAT, while flux trace variables (flux, mass release and mass release rate) are written to TRACEFXD.DAT. The format is:

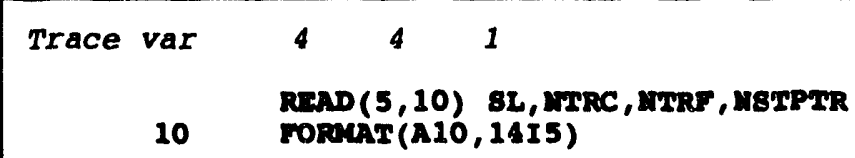


If the number of concentration traces, NTRC, is non-zero, the nodal locations for the concentration traces are read by the code. These variables are read through the subroutine INPTI which reads integer values using the following format.

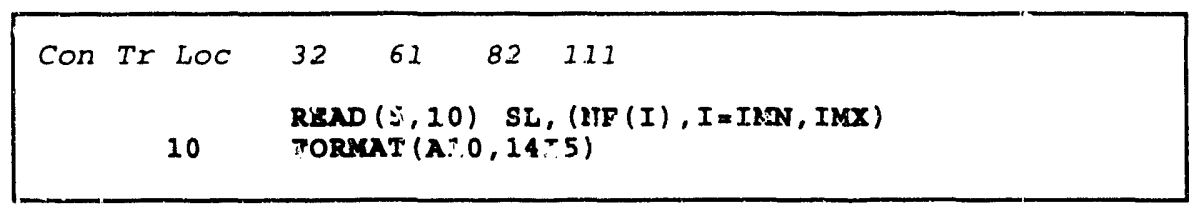
NTRCEC.

In the calling statemant for INPTI, the dummy array NF is assigned to the array

Similarly, if the number of fluis traces, NTRF, is non-zero, the trace locations are read in by the code using the INPTI subroutine while using the array NF to store values for NTRCEF.

For the MCMC model, the DUST code forces the flux and concentration traces to occur at the same location. If the input deck, specifies different locations, the code over rides the input values. This is done because the MCMC model only performs calculations at the points specified through the concentration trace locations.

\subsection{DATA SET 5: Facility Co-ordinate Data (Menu 5)}

The first two cards in this Data Set are the label cards for the end of the previous set and the beginning of this set. The next card is required by both transport models and contains the height and cross sectional area of the facility. The height of the facility is calculated when using the FD model and may be input as zerc. The format for these cards are:

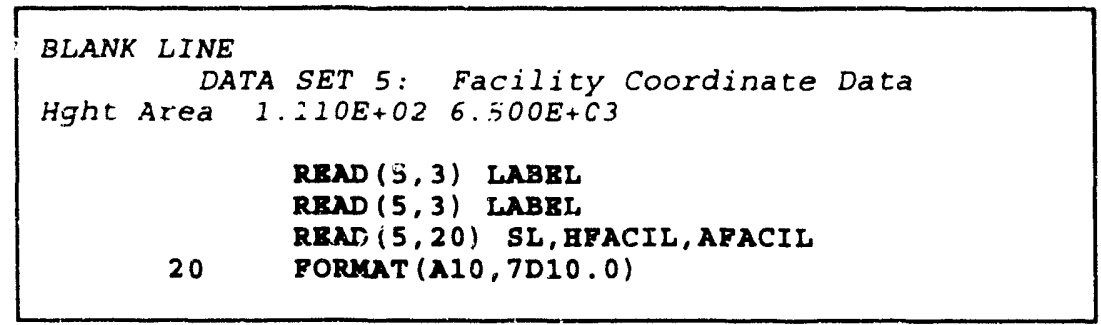

If the MCMC model is used, this data set is complete. The size of each computational cell is determined from the facility heighi and number of cells. If the FD model is used, the size of each cell must be specified. The procedure used to accomplish this is encoded in the subroutine READR. The format statement in READR is: 


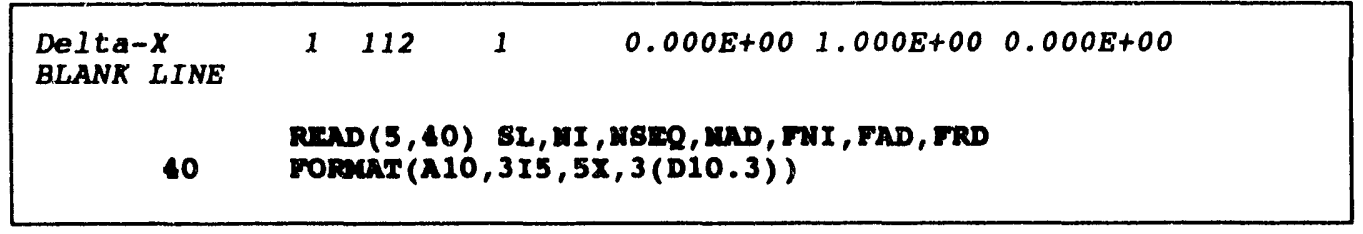

In the example presented in Table 7.1, the user spaced the location of all 112 control volumes $1 \mathrm{~cm}$ apart with the following card:

$$
\text { Delta-X } \quad \begin{array}{lllllll}
1 & 112 & 1 & 0.000 \mathrm{E}+00 & 1.000 \mathrm{E}+00 & 0.000 \mathrm{E}+0
\end{array}
$$

A blank card follows, signifying the end of the input to subroutine READR.

This places the first control volume between 0 and $0.5 \mathrm{~cm}$, the second between 0.5 and $1.5 \mathrm{~cm}$, etc. The last control volume is between $110.5 \mathrm{~cm}$ and $111 \mathrm{~cm}$. A complete discussion of the geometry definition for the FD model is in Appendix A.

\subsection{DATA SET 6: Initial and Boundary Conditions (Menu 6)}

The first two cards in this data set are label cards which indicate the completion of the previous data set and the beginning of this data set. The next set of cards are required by both transport models and are read by the subroutine READR and define the initial conditions for each computational pcint. The READ statements are:

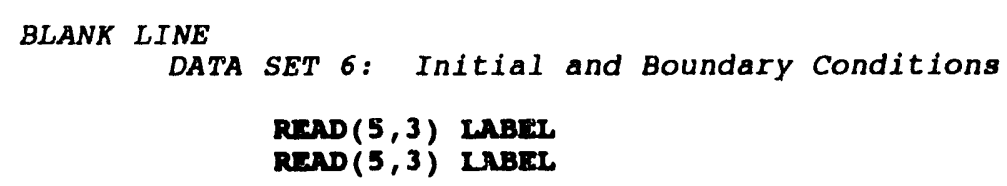

The format statement in subroutine READR is:

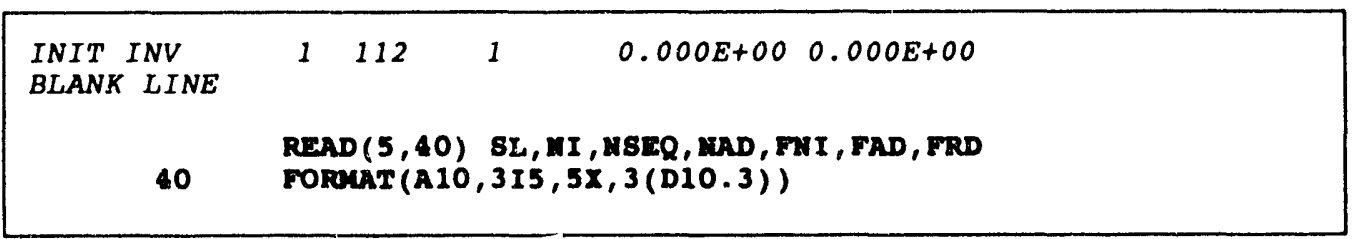

In this case, the values input into READR for FNI, FAD, and FRD are used to determine concentrations. 
Within READR, the READ statement is repeated until the value for NI is zero. Therefore, to end this segment of input a blank card is used. Again, the DUST code checks to insure that all locations are given an initial value. Example initial conditions are provided in the sample input decks, Table 7.1 and 7.2.

If the MCMC model is used, boundary conditions are not required and this Data Set is complete. However, if the FD model is used, the code user must specify the choice for the boundary condition at both boundaries. Four different boundary conditions (concentration, total flux, advective flux, and dispersive flux, respectively) may be selected in the parameter IBFLAG. The boundary conditions are determined using a table with values that depend on time. The units used for the values depend on the boundary condition flag. However, if the mass flag (Menu 1) is set to indicate that mass values are input in Curies, the boundary conditions mass units must be Curies. The first input card defines the flags used to determine the boundary condition and the number of points used to specify the boundary condition in the tables.

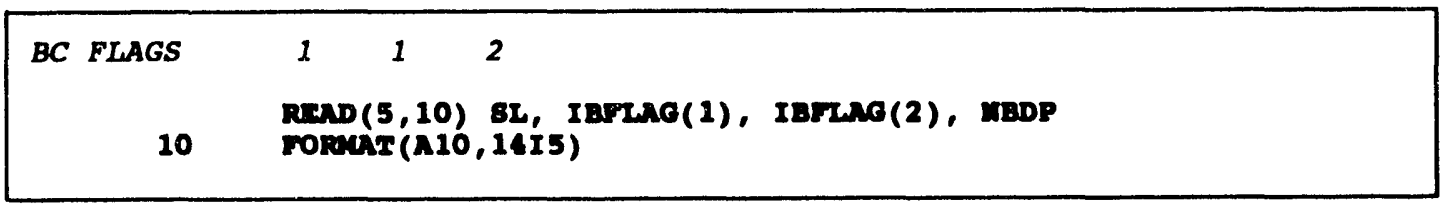

The tables of boundary condition versus time are specified using the subroutine INPTR2. INPTR2 is called to read the times for the top boundary (node 1) condition followed by the values at the top. Then, the times for the bottom boundary followed by the values at the bottom. INPTR2 is called by the following statement:

\section{CALL INPTR2(TBC,MXBDP,NBDP,I,MXBPR)}

In this example, TBC, time of the boundary condition is specified through input. Other variables in the call statement are MXBDP, the maximum boundary data points in a Table (currently 10), NBDP is the user specified number of points in the table, I is the boundary condition indicator ( $I=1$, top boundary; $I=2$, bottom boundary), and MXBPR is the maximum number of boundaries (currently 2).

INTPR2 uses the following read statement:

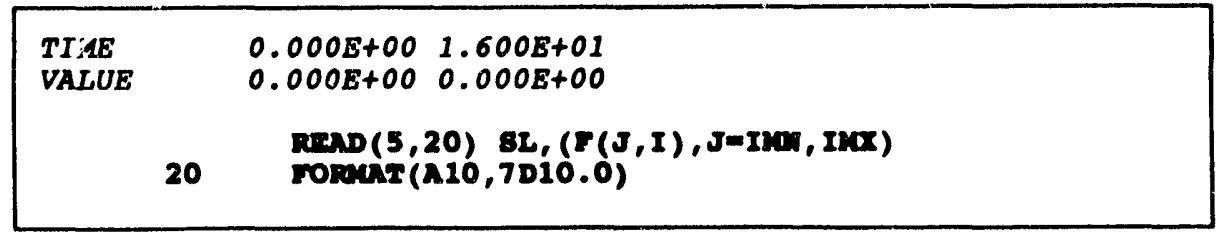


At this point, the procedure is repeated for the bottom boundary condition values.

An example of specifying both the top and bottom boundary concentrations to be zero for the duration of the problem is provided in Table 7.1.

\subsection{DATA SET 7: Water Flow Parameters (Menu 7)}

The first two cards in this data set are label cards which indicate the completion of the previous data set and the beginning of this data set. They are:

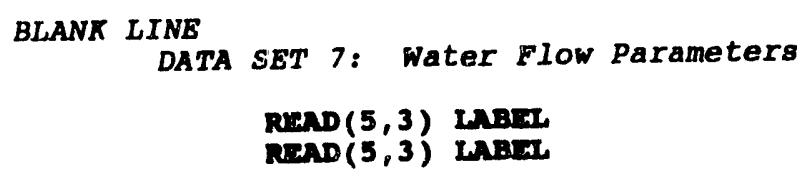

\section{$\underline{\text { MCMC Model }}$}

If the MCMC model is used, the Darcy velocity and the moisture content are single valued parameters which are input using the following statements:

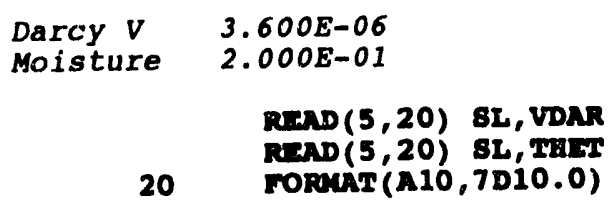

At this point, this Data Set is complete for the MCMC model.

\section{$\underline{\text { FD Model }}$}

If the FD model is used, the Darcy velocity can be a function of time and is determined by tabular input similar to that used to specify the boundary conditions. The moisture content may vary with location and therefore it is input similar to the approach used in specifying location.

The first data card for the FD model specifies the number of points in the Darcy velocity versus time table and is: 


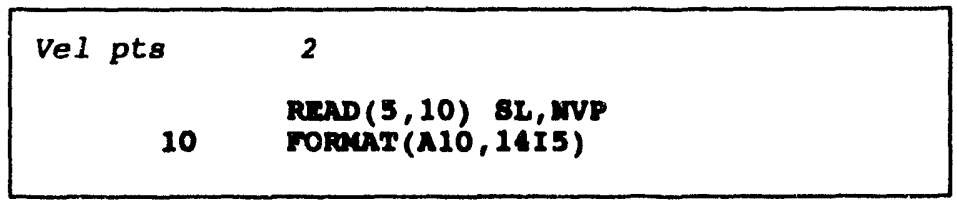

The tables for Darcy velocity are read using the subroutine INPTR2 which was described in the beginning of this chapter and in the discussion of boundary conditions, Section \%.6. The times used in the table are read in first, seven to a card beginning in column 11. After all the times are input, the Darcy velocity values are read. The example provided in Table 7.1 requires two values for the velocity table and defines the Darcy velocity to be $3.6 \mathrm{E}-6 \mathrm{~cm} / \mathrm{s}$ for the first 17 years (maximum problem time is 16 years).

The moisture content is input using the subroutine READR. Use of this subroutine is also described in the beginning of this chapter. The format used in the read statement is:

\begin{tabular}{|c|c|c|}
\hline \multirow[t]{2}{*}{$\begin{array}{l}\text { MST CONT } \\
\text { BLANK LINE }\end{array}$} & 1112 & $2.000 E-010.000 E+00$ \\
\hline & $\begin{array}{l}\operatorname{READ}(5,40) \\
\operatorname{POR}(\mathbf{A 1 0}, 3\end{array}$ & $\begin{array}{l}\text { SL, MI , MSEQ, wAD, FUI, FAD, FRD } \\
315,5 x, 3(D 10.3))\end{array}$ \\
\hline
\end{tabular}

Here, the variables FNI, FAD, FRD refer to moisture content. In the example provided in Table 7.1, the moisture content is set to 0.2 at all 112 points. READR continues reading cards until NI is zero. This is accomplished in the example through a blank card as shown above.

\subsection{DATA SET 8: Container Parameters (Menu 8)}

As usual, the first two cards in this data set are label cards which indicate the completion of the previous data set and the beginning of this data set. They are:

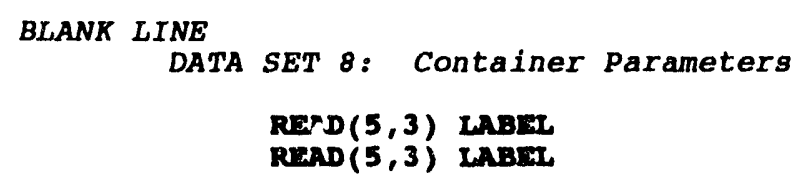

The next card specifies the number of containers and container types to be modeled. The code is dimensioned to allow up to 300 containers and 20 container types. A container is used to define a location at which a wasteform resides. Each container may have a unique time of failure (time at which the container no longer provides any barrier to radionuclide release). The number of container types indicates the number of different local failure rates. If local failure 
is not modeled, NCTYPE should be 1 (a value of 0 will be set to 1 by the code). The read statement is:

\begin{tabular}{|c|c|}
\hline NCON TYPE & 53 \\
\hline 10 & $\begin{array}{l}\operatorname{READ}(5,10) \text { BL, } \\
\operatorname{rOmeON}(\mathrm{A10}, 1415)\end{array}$ \\
\hline
\end{tabular}

After these values are defined, the failure time (in years) for each container must be specified. This is accomplished in subroutine INPTR with the following statement:

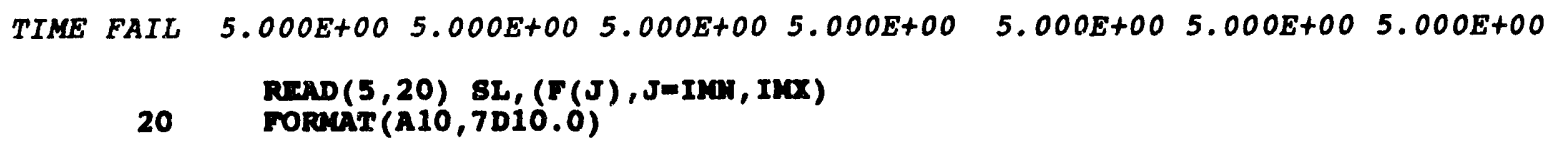

The dummy array $\mathrm{F}$ represents the time to failure, TIMEF in this call to INTPR. In the examples of Table 7.1 and 7.2 there are 53 containers, therefore, there are eight container failure time cards. The first seven contain seven failure times and the last one contains only four failure times.

\section{$\underline{\text { MCMC Model }}$}

At this point, the input requirements for the MCMC and FD models differ. For the MCMC model, the only remaining parameter to specify is the location (by mixing cell number) of each container. Container locations are specified using the subroutine INPTI. Each container must be given a unique location. In the example, there are 53 container locations specified by use of the following:

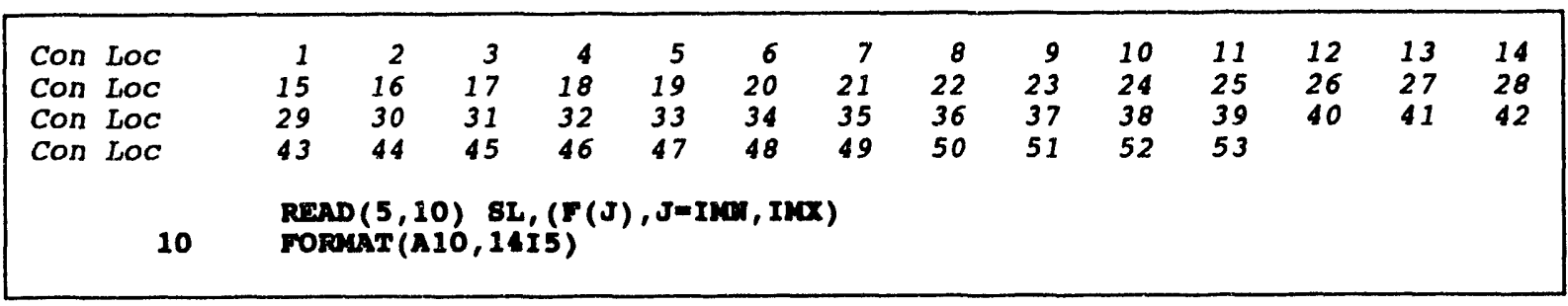

Here, the array $F$ is used to define NELCON. For 53 containers, four input cards are required (three containing 14 values and one containing 11 ). 
After completing the container location specification this Data Set is complete when the MCMC model is used.

\section{FD Model}

The FD model also permits localized (partial) failure of the containers. This requires further input. After specifying the time of failure, the user must specify if local failure is modeled for each separate container type (recall the minimum number of container types is 1 ). This is accomplished using the subroutine INPTI.

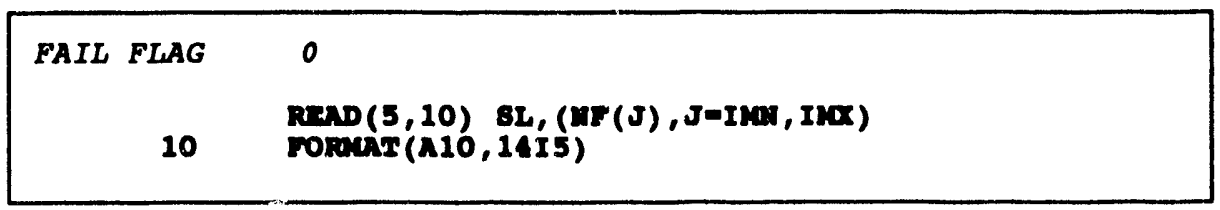

The dummy array NF is used to define the array IPIT.

With the array IPIT, the code cycles through each container type. If IPIT(J) is 1 , the flag indicates that local failure is modeled for the J-th container type. In this case, the parameters (defined in the discussion of Menu 8 in Chapter 6) that predict local corrosion must be input. Suggested values for these parameters are presented in Appendix C. The read statement is:

If IPIT(J) is zero, the previous statement is not executed and input is not required. Therefore, to model only general corrosion, the user can set the number of container types, NCTYPE, to 1 and set IPIT(1) to zero. This was done in the example, Table 7.1. If the user desired to use localized corrosion on only a few containers, this could be accomplished by defining two container types. Container type 1 would have IPIT(1) equal zero and therefore would consider only general corrosion. Container type 2 would have IPIT( 2$)=1$ and the local corrosion variables would be input only for this container.

After specifying the local container parameters, the location of each container must be specified. This is accomplished in the using subroutine INPTI in the same manner as for the MCMC model. The reader is referred to that section if clarification is needed. 
When more that one container types is defined, the user must specify the type of container in each container location. This is also accomplished using the subroutine INPTI. If only one container type is defined, the code automatically sets this parameter and input is not required. Specifying wasteform types requires an identical procedure. An example of this procedure is provided in Section 7.9.

\subsection{DATA SET 9: Wasteform Leaching Parameters (Menu 9)}

The first two cards in this data set are the label cards used to indicate the completion of the previous set and the beginning of this set. The third card contains the number of different waste types, currently 20 is the maximum number of waste types. The DUST code assumes that there is one wasteform in each container. A waste type defines a unique set of release parameters. The MCMC and FD models both permit multiple release rate parameters. The first three input cards for this data set are:

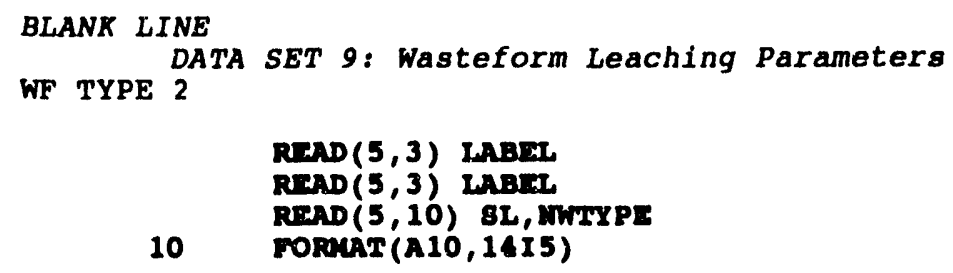

At this point, the input requirements for the MCMC and FD models differ. As such, they are discussed separately.

\section{MCMC Model}

This model requires only two wasteform release parameters for each waste type, the rinse release fraction and the yearly fractional release rate. The fraction available for uniform release is determined as 1.0 minus the rinse release fraction. The input routine first reads a label card containing identifiers for the variables, it then reads one data card per wasteform type. In the example in Table 7.2, there are two waste types.

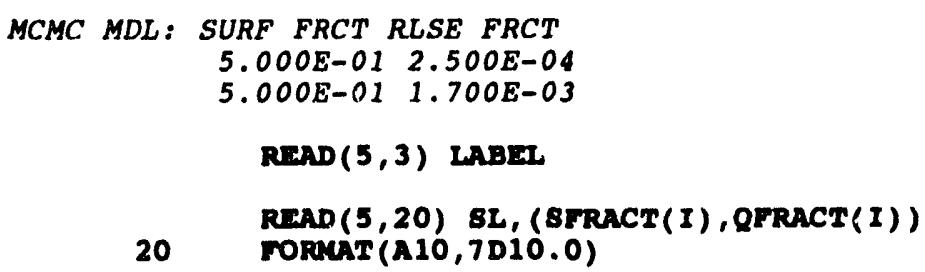


After defining the release parameters, the inventory for all NCON containers must be specified. Each container may have a unique inventory. This is accomplished using the subroutine INPTR which contains the following read statement:

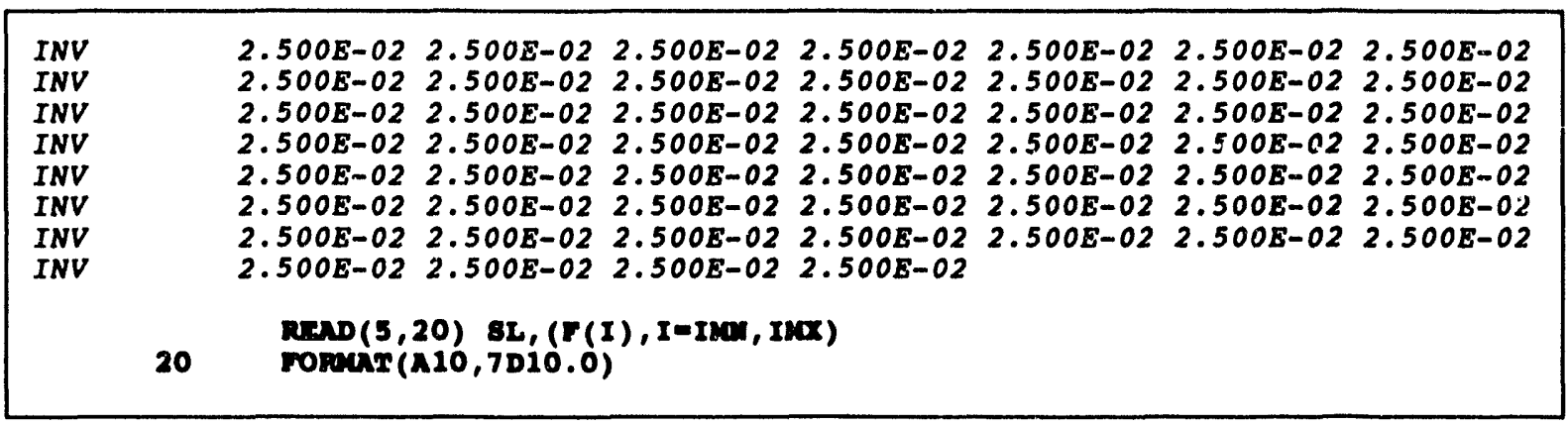

The dummy array $\mathrm{F}$ is used to determine the variable WTINIT used by DUST. This card is repeated until all NCON values for initial mass are specified.

The final input requirement is the assignment of wasteform release parameters to each container. This is only necessary if the number of waste types is greater than 1 . When required, the subroutine INPTI is used to read the input as follows:

\begin{tabular}{|c|c|c|c|c|c|c|c|c|c|c|c|c|c|c|c|}
\hline $\begin{array}{l}\text { Waste } \\
\text { Waste } \\
\text { Waste } \\
\text { Waste }\end{array}$ & $\begin{array}{l}\text { Typ } \\
\text { TYp } \\
\text { TYp } \\
\text { Typ }\end{array}$ & $\begin{array}{l}1 \\
1 \\
2 \\
2\end{array}$ & $\begin{array}{l}1 \\
1 \\
2 \\
2\end{array}$ & $\begin{array}{l}1 \\
1 \\
2 \\
2\end{array}$ & $\begin{array}{l}1 \\
1 \\
2 \\
2\end{array}$ & $\begin{array}{l}1 \\
1 \\
2 \\
2\end{array}$ & $\begin{array}{l}1 \\
1 \\
2 \\
2\end{array}$ & $\begin{array}{l}1 \\
2 \\
2 \\
2\end{array}$ & $\begin{array}{l}1 \\
2 \\
2 \\
2\end{array}$ & $\begin{array}{l}1 \\
2 \\
2 \\
2\end{array}$ & $\begin{array}{l}1 \\
2 \\
2 \\
2\end{array}$ & $\begin{array}{l}1 \\
2 \\
2 \\
2\end{array}$ & $\begin{array}{l}1 \\
2 \\
2\end{array}$ & $\begin{array}{l}1 \\
2 \\
2\end{array}$ & $\begin{array}{l}1 \\
2 \\
2\end{array}$ \\
\hline & 10 & $\begin{array}{l}R \mathbf{N} \\
\mathbf{r}\end{array}$ & $\mathbf{T}(2)$ & & & & $=I \times 1$ & Ixx) & & & & & & & \\
\hline
\end{tabular}

The dummy array NF is used to determine the variable IWTYPE used by DUST. The read statement is repeated until IMX $=$ NCON, the number of containers specified.

At this point, the input for the MCMC model is complete.

\section{FD Model}

The FD model is more general than the MCMC model and therefore requires more input. After reading the number of waste types the FD model requires a geometry flag for each waste type. This flag is only used when diffusion controlled release from the wasteform is modeled, however, this input is always required. A value of 0 indicates cylindrical geometry; 1 indicates rectangular geometry. INPTI is used to read in the values for the geometry flag, IDIFF. 


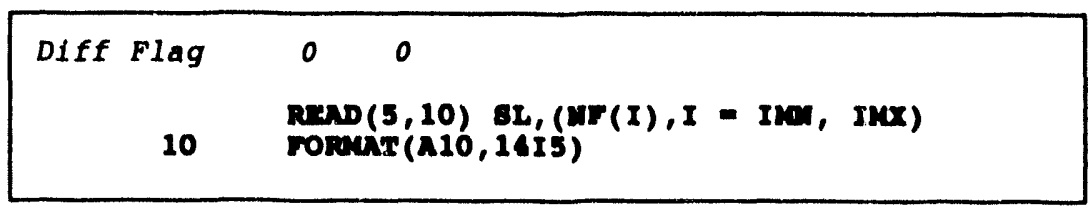

The read statement is repeated until IMX equals the number of waste types. The dummy array NF is used to assign valued to the array IDIFF used in DUST.

After specifying the geometry, the release parameters are input. The input routine reads a label card with identifiers for the parameters. Then the code reads one card per wasteform type with the specified values.

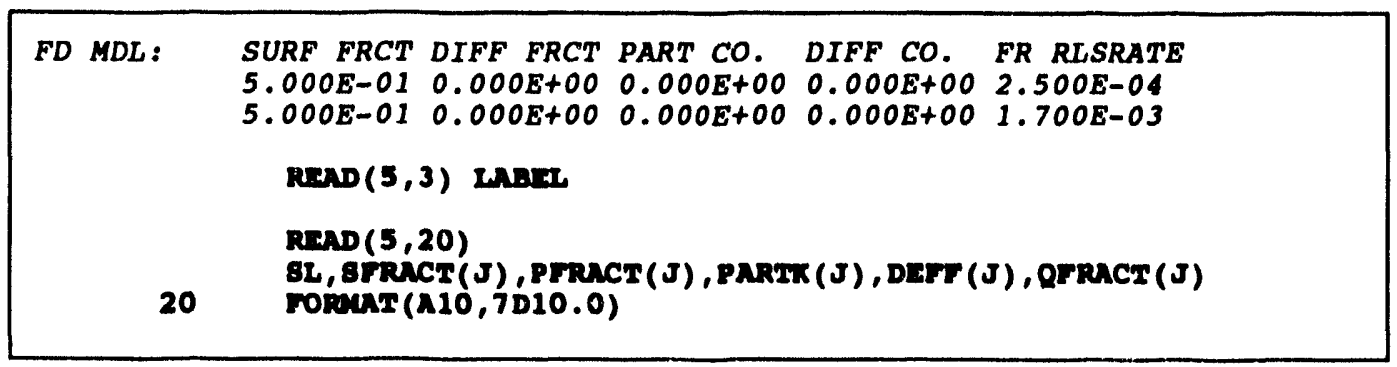

The variables are:

SFRACT, fraction available for surface rinse;

PFRACT, fraction available for diffusion release;

PARTK, the wasteform partition coefficient;

DEFF, the wasteform diffusion coefficient; and

QFRACT, the annual fractional release rate.

These variables have been discussed in detail in Chapter 6. In Table 7.1, there are two waste types therefore, there are two sets of release parameters.

After defining the release parameters, wasteform dimensions are required as input. These dimensions are required for calculating diffusive releases. If diffusion release is not modeled, wasteform dimensions are still required input. However, they may be input as zero. The input routine requires a label card to identify the wasteform dimensions required. This is followed by the input data for dimensions. 


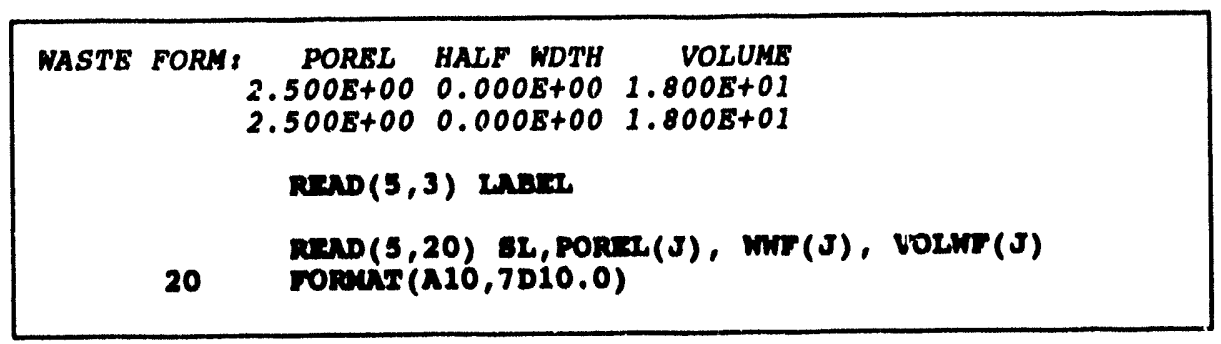

The previous statement is executed once for each waste type. The variables are:

POREL, the radius (cylindrical geometry) or half-height (rectangular geometry);

WWF, the half-width in rectangular geometry, unused for cylindrical geometry; and VOLWF, the volume of the wasteform.

These variables are used to calculate all geometric quantities needed by the analytical diffusion release models.

At this point, the input procedure is identical to that used for the MCMC model after specifying release parameters. The initial mass in each container, WTINIT, is specified using the subroutine INPTR and the wasteform type for each container, IWTYPE, is specified using subroutine INPTI. The reader is referred to the discussion in this section on the MCMC model for a complete description of this process.

\subsection{DATA SET 10: External Sources (FD model only)}

If the MCMC model is specified, input is not required for this section. The FD model permits an external source which is defined through tabular input of source strength versus time. The first two cards in the data set are label cards identifying the end of the previous data set and the beginning of this set. The third card specifies the total number of sources, the number of different source profiles, and the number of data points required to create the table of source strength versus time. The read statements are:

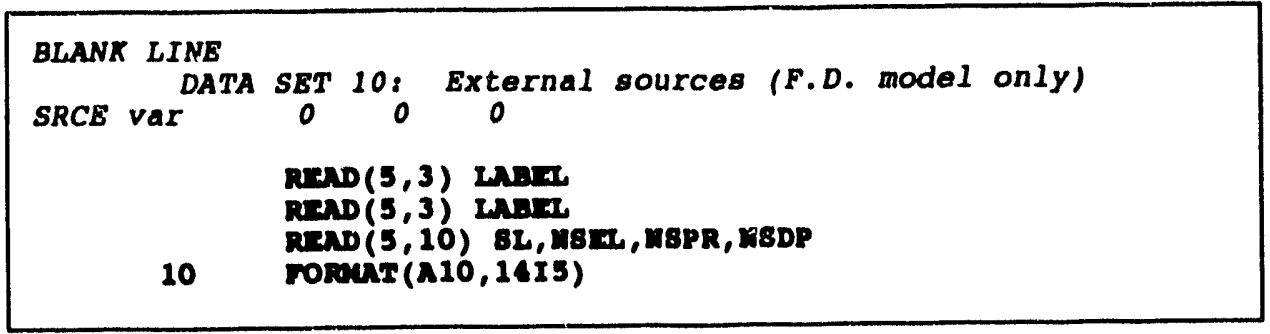


If the number of sources, NSEL, is zero, the required input is complete. This is the case in the example input deck, Table 7.1. If there are external sources, input to define the table of source strength versus time, the location of the sources, and which profile to use at each source location are required.

The table of source strength versus time is input for each different source profile. The DUST code uses the subroutine INPTR2 to translate the input into the variables required by DUST. The read statement in INPTR2 is:

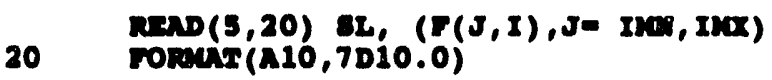

DUST calls INPTR2 to obtain the times in the external source table for table number I. After obtaining the times for profile, I, the source strength is obtained using INPTR2. If the mass/activity flag requires mass units of curies, the source should be input in curies $/ \mathrm{cm}^{3} / \mathrm{s}$. The code will translate this to grams $/ \mathrm{cm}^{3} / \mathrm{s}$ internally. After completing a profile, the index I is increased and the procedure repeated until all profiles are defined.

After completing the profiles, the source locations are specified. Source locations are defined using the same procedure when defining container locations. The subroutine INPTI is used to read in the locations as defined by control volume number.

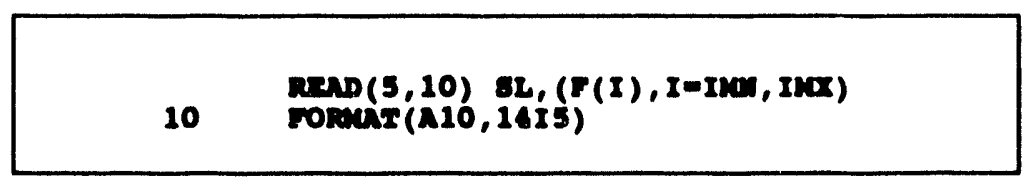
DUST.

In this case, the dummy array $F$ is used to assign values to the array LSRC used by

The final piece of input required is the assignment of source type to each source location. The input is similar to specifying container and wasteform types. The routine INPTI is again used. In this case, the dummy variable $\mathrm{F}$ represents the variable ISTYPE.

An example of the input required for specifying an external source is in Table 7.3. In this example, there are 3 source nodes, 2 source types, 4 data points in each table, the sources are located in nodes 20,30 and 40 , and nodes 20 and 40 use profile 1 , while node 30 uses source type 2. The type 1 source release rate is $1 \mathrm{E}-5$ for 0 to 10 years. After 10.01 years the release rate is zero. The type 2 source releases nothing for the first 10 years, increases to $1 \mathrm{E}-5$ after 10.0 years and remains constant until 1000 years. Linear interpolation is used to obtain source 
strength between data points in the table. If the value of time during the computation lies outside the range in the table, unpredictable results may occur.

\begin{tabular}{|c|c|c|c|c|}
\hline Table & Input $\mathbf{c}$ & cards required to & pecify an exte & nal source \\
\hline $\begin{array}{l}\text { SRCE var } \\
\text { TIME } \\
\text { VALUE } \\
\text { TIME } \\
\text { VALUE } \\
\text { SRC LOC } \\
\text { SRC type }\end{array}$ & $\begin{array}{c}3 \\
0.000 \mathrm{E}+00 \\
1.000 \mathrm{E}-05 \\
0.000 \mathrm{E}+00 \\
0.000 \mathrm{E}+00 \\
203 \\
1\end{array}$ & $\begin{array}{lc}2 & 4 \\
1.000 E+01 \\
1.000 E-05 \\
1.000 E+01 \\
0.000 E+00 \\
30 & 40 \\
2 & 1\end{array}$ & $\begin{array}{l}1.001 \mathrm{E}+01 \\
0.000 \mathrm{E}+00 \\
1.001 \mathrm{E}+01 \\
1.000 \mathrm{E}-05\end{array}$ & $\begin{array}{l}1.000 \mathrm{E}+03 \\
0.000 \mathrm{E}+00 \\
1.000 \mathrm{E}+03 \\
1.000 \mathrm{E}-05\end{array}$ \\
\hline
\end{tabular}




\section{DUST OUTPUT FILES}

DUST creates a number of output files that are useful for tracking the predictions of the models. While running the DUST code, the code prompts the user for the name of the primary output file. This file contains an echo check of the input as well as the output requested in the input file. In addition, up to five other files are written by the DUST code. These files include: TRACECND.DAT, TRACEFXD.DAT which may be written with either the MCMC or FD transport model, and CONCNT.DAT and LEACHRL.DAT which are written only if the FD transport model is used. The content of each of file, along with a small example, is presented in this chapter.

\subsection{Primary Output File}

Table 8.1 contains an abbreviated output file for the FD test problem presented in Chapter 5 that was used for comparing the MCMC and FD models. In this problem there were 12 containers with failure times ranging from 0 to 40 years. Containers were located in every other node beginning at node 11 and ending at node 33 . Each node was $100 \mathrm{~cm}$. in length. Release from each wasteform was modeled assuming that the amount available for rinse and diffusion release was zero. The fractional release rate for all wasteforms was $5 \%$ per year. The initial condition was zero concentration everywhere. The boundary conditions were zero concentration at each boundary.

This main output file writes all information within 80 columns. This was done to provide a convenient method of viewing the files on standard desktop video display units.

The beginning of this file provides an echo check of the input file. Each of the ten data sets are printed for review. The data sets are: (1) Problem title and definition; (2) Time parameters; (3) Material properties; (4) Output control parameters, including trace locations; (5) Nodal coordinates and facility dimensions; (6) Initial and Boundary Conditions; (7) Water flow parameters (Darcy velocity and moisture content); (8) Container parameters; (9) Wasteform parameters; and (10) Source/Sink parameters. In the output, all automatic generation of initial conditions, node locations, and moisture content are expanded to print the specified value at each location as seen in Table 8.1. The structure of this file is identical for the MCMC model, however, the details are slightly different due to the different input requirements of the models.

After completion of the input check, the code prints a message that input is complete and that the calculation is about to begin. At this point, output is printed every time step at which the input value for KPR is non-zero. In this example, KPR for the first time step was 3. Therefore, a full output of concentration, flux, facility mass release rate, container performance and wasteform release is printed. 
Table 8.1 rypical primary output file when the FD transport model is used

TITLE: FD MODEL - H-3, IK=0.2, VDAR=1.58E-6, MLT CONT

PROBLEA DEFINITION ***

RADIONUCLIDE. . . . . . . . . . H-3

HALF-LIFE (YRS) ............ . $1.23 \mathrm{E}+01$

SATURATION CONCENTRATION.:.:. $1.00 \mathrm{E}+01$

ATOMIC WEIGHT . . . . . . . . . . . . 3.0

ACTIVITY FLAG (0-GRANS, i=ACTIVITY). - 1

MURBER OF NODAL POINTS/MIXING CELLS . . 50

TRNSPRT FLAG (MIX CELL=0,FIN DIFF=1). . 1

\#*** TIME PARAMETERS *** *

NUMBER OF TIME INCREAENTS .. . . . . 90

WO. OF DISCRETE TIME CHAMGES. . . . . . 1

TIME IMCREAENT (DELT - YEAKS) . . . . 1.00E+00

MULTIPLIER FOR IMCREASING DELT. . . . 0.00B+00

MAXIMUA VALUE OF DELT (YEARS) . . . . 1.008+00

MAXIMUM VALUE OF TIME (YEARS) . . . $1.00 \mathrm{E}+03$

LIST OF TIES AT MHICH 'DELT' CHANGES:

NO. TIME (YRS) 30. TIME (YRS) NO. TIME (YRS) NO. TIME (YRS)

i. $1.00 \mathrm{E}+03$

YO. TIYTE (YRS) ( NO. TIME (YRS)

** material properties ***

MUMBER OF MATERIALS

NUMBER OF REDEFINED MATERIAJS . . . . 0

$\begin{array}{cclll}\text { MAT. HO. DISTK.COEFF. } & \text { DENSITY } & \text { DISPERS. } & \text { DIFFUSION } \\ 1 & 1.00 \mathrm{E}+00 & 1.60 \mathrm{E}+00 & 0.00 \mathrm{E}+00 & 0.00 \mathrm{E}+00\end{array}$

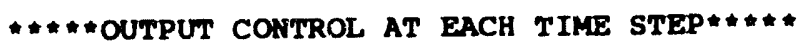

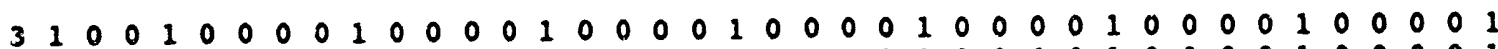

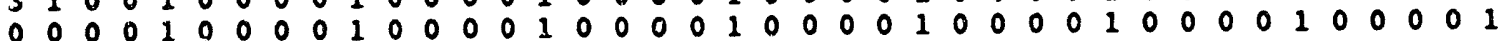

$0 \begin{array}{llllllllllll}0 & 0 & 0 & 0 & 1 & 0 & 0 & 0 & 0 & 1\end{array}$ 
Table 8.1 Typical prinary output file when the FD transport model 18 used

\begin{tabular}{|c|c|c|c|c|c|c|c|c|c|c|}
\hline $\begin{array}{c}\text { NODE } \\
11\end{array}$ & $\begin{array}{c}\text { NUMBERS } \\
17\end{array}$ & $\begin{array}{l}\text { FOR } \\
22\end{array}$ & $\begin{array}{c}\text { CONCEN } \\
33\end{array}$ & $\begin{array}{c}\text { NTRATION } \\
44\end{array}$ & RACES : & & & & & \\
\hline $\begin{array}{l}\text { NODE } \\
11\end{array}$ & $\begin{array}{c}\text { NUMBERS } \\
17\end{array}$ & $\begin{array}{l}\text { FOR } \\
22\end{array}$ & $\begin{array}{r}\text { FLUX } \\
33\end{array}$ & $\begin{array}{c}\text { TRACES : } \\
44\end{array}$ & & & & & & \\
\hline$\star \star \star \star \star$ & NODE CC & OORD & INATES & $\star \star \star \star$ & & & & & & \\
\hline $\begin{array}{r}\text { NODE } \\
1 \\
6 \\
11 \\
16 \\
21 \\
26 \\
31 \\
36 \\
41 \\
46\end{array}$ & $\begin{array}{r}C 0 O 1 \\
0 \\
500 \\
1000 \\
1500 \\
2000 \\
2500 \\
3000 \\
3500 \\
4000 \\
4500\end{array}$ & $\begin{array}{l}\text { RD. } \\
.0 \\
.0 \\
.0 \\
.0 \\
.0 \\
.0 \\
.0 \\
.0 \\
.0 \\
.0\end{array}$ & $\begin{array}{r}\text { NODE } \\
2 \\
7 \\
12 \\
17 \\
22 \\
27 \\
32 \\
37 \\
42 \\
47\end{array}$ & $\begin{array}{r}\text { COORD. } \\
100.0 \\
600.0 \\
1100.0 \\
1600.0 \\
2100.0 \\
2600.0 \\
3100.0 \\
3600.0 \\
4100.0 \\
4600.0\end{array}$ & $\begin{array}{r}\text { NODE } \\
3 \\
8 \\
13 \\
18 \\
23 \\
28 \\
33 \\
38 \\
43 \\
48\end{array}$ & $\begin{array}{l}\text { COORD. } \\
200.0 \\
700.0 \\
1200.0 \\
1700.0 \\
2200.0 \\
2700.0 \\
3200.0 \\
3700.0 \\
4200.0 \\
4700.0\end{array}$ & $\begin{array}{r}\text { NODE } \\
4 \\
9 \\
14 \\
19 \\
24 \\
29 \\
34 \\
39 \\
44 \\
49\end{array}$ & $\begin{array}{r}\text { COORD. } \\
300.0 \\
800.0 \\
1300.0 \\
1800.0 \\
2300.0 \\
2800.0 \\
3300.0 \\
3800.0 \\
4300.0 \\
4800.0\end{array}$ & $\begin{array}{r}\text { NODE } \\
5 \\
10 \\
15 \\
20 \\
25 \\
30 \\
35 \\
40 \\
45 \\
50\end{array}$ & $\begin{array}{r}\text { COORD. } \\
400.0 \\
900.0 \\
1400.0 \\
1900.0 \\
2400.0 \\
2900.0 \\
3400.0 \\
3900.0 \\
4400.0 \\
4900.0\end{array}$ \\
\hline
\end{tabular}

FACILITY DIMENSIONS

FACILITY HEIGHT X-SECT. AREA VOL.OF FACILITY

$\begin{array}{rrr}4.900 \mathrm{E}+03 & 9.850 \mathrm{E}+03 & 4.826 \mathrm{E}+07\end{array}$

INITIAL CONCENTRATION AT EACH NODE:

NODE CONCENTR. NODE CONCENTR. NODE CONCENTR. NODE CONCENTR. NODE CONCENTR.

\begin{tabular}{|c|c|c|c|c|c|c|c|c|}
\hline $\begin{array}{rl}1 & 0.0 \mathrm{E}+00 \\
6 & 0.0 \mathrm{E}+00 \\
11 & 0.0 \mathrm{E}+00 \\
16 & 0.0 \mathrm{E}+00 \\
21 & 0.0 \mathrm{E}+00 \\
26 & 0.0 \mathrm{E}+00 \\
31 & 0.0 \mathrm{E}+00 \\
36 & 0.0 \mathrm{E}+00 \\
41 & 0.0 \mathrm{E}+00\end{array}$ & $\begin{array}{r}2 \\
7 \\
12 \\
17 \\
22 \\
27 \\
32 \\
37 \\
42\end{array}$ & $\begin{array}{l}0.0 \mathrm{E}+00 \\
0.0 \mathrm{E}+00 \\
0.0 \mathrm{E}+00 \\
0.0 \mathrm{E}+00 \\
0.0 \mathrm{E}+00 \\
0.0 \mathrm{E}+00 \\
0.0 \mathrm{E}+00 \\
0.0 \mathrm{E}+00 \\
0.0 \mathrm{E}+00 \\
0.0 \mathrm{E}+00\end{array}$ & $\begin{array}{r}3 \\
8 \\
13 \\
18 \\
23 \\
28 \\
33 \\
38 \\
43 \\
48\end{array}$ & $\begin{array}{l}0.0 \mathrm{E}+00 \\
0.0 \mathrm{E}+00 \\
0.0 \mathrm{E}+00 \\
0.0 \mathrm{E}+00 \\
0.0 \mathrm{E}+00 \\
0.0 \mathrm{E}+00 \\
0.0 \mathrm{E}+00 \\
0.0 \mathrm{E}+00 \\
0.0 \mathrm{E}+00 \\
0.0 \mathrm{E}+00\end{array}$ & $\begin{array}{r}4 \\
9 \\
14 \\
19 \\
24 \\
29 \\
34 \\
39 \\
44 \\
49\end{array}$ & $\begin{array}{l}0.0 \mathrm{E}+00 \\
0.0 \mathrm{E}+00 \\
0.0 \mathrm{E}+00 \\
0.0 \mathrm{E}+00 \\
0.0 \mathrm{E}+00 \\
0.0 \mathrm{E}+00 \\
0.0 \mathrm{E}+00 \\
0.0 \mathrm{E}+00 \\
0.0 \mathrm{E}+00 \\
0.0 \mathrm{E}+00\end{array}$ & $\begin{array}{l}5 \\
10 \\
15 \\
20 \\
25 \\
30 \\
35 \\
40 \\
45 \\
50\end{array}$ & $\begin{array}{l}0.0 \mathrm{E}+00 \\
0.0 \mathrm{E}+00 \\
0.0 \mathrm{E}+00 \\
0.0 \mathrm{E}+00 \\
0.0 \mathrm{E}+00 \\
0.0 \mathrm{E}+00 \\
0.0 \mathrm{E}+00 \\
0.0 \mathrm{E}+00 \\
0.0 \mathrm{E}+00 \\
0.0 \mathrm{E}+00\end{array}$ \\
\hline
\end{tabular}


Table 8.1 Typical primary output file when the FD transport model is used

$\star \star \star \star$ BOUNDARY CONDITIONS $\star \star \star \star ~$

FLAGS FOR BOUNDARY CONDITIONS:

TOP BOUNDARY: FLAG SPECIFIED IS 1: Concentration

BOTTOM BOUNDARY: FLAG SPECIFIED IS 1: Concentration

NO. OF BOUND. COND. DATA POINTS IN EACH PROFILE: 2

TOP BOUNDARY CONDITION:

TIME (YRS) VALUE

$0.000 \mathrm{E}+00 \quad 0.000 \mathrm{E}+00$

$1.000 \mathrm{E}+03 \quad 0.000 \mathrm{E}+00$

BOTTOM BOUNDARY CONDITION :

$\begin{array}{cc}\text { TIME (YRS) } & \text { VALUE } \\ 0.000 \mathrm{E}+00 & 0.000 \mathrm{E}+00 \\ 1.000 \mathrm{E}+03 & 0.000 \mathrm{E}+00\end{array}$

WATER FLOW PARAMETERS

DARCY VELOCITY TABLE (CM/SEC):

$\begin{array}{rr}\text { TIME (YRS) } & \text { VDAR } \\ 0.000 \mathrm{E}+00 & 1.587 \mathrm{E}-06 \\ 1.001 \mathrm{E}+03 & 1.587 \mathrm{E}-06\end{array}$

$1.001 \mathrm{E}+03 \quad 1.587 \mathrm{E}-06$

MOISTURE CONTENT

$\begin{array}{rrrrrrrrrr}\text { NODE } & \text { THETA } & \text { NODE } & \text { THETA } & \text { NODE } & \text { THETA } & \text { NODE } & \text { THETA } & \text { NODE } & \text { THETA } \\ 1 & 0.20 & 2 & 0.20 & 3 & 0.20 & 4 & 0.20 & 5 & 0.20 \\ 6 & 0.20 & 7 & 0.20 & 8 & 0.20 & 9 & 0.20 & 10 & 0.20 \\ 11 & 0.20 & 12 & 0.20 & 13 & 0.20 & 14 & 0.20 & 15 & 0.20 \\ 16 & 0.20 & 17 & 0.20 & 18 & 0.20 & 19 & 0.20 & 20 & 0.20 \\ 21 & 0.20 & 22 & 0.20 & 23 & 0.20 & 24 & 0.20 & 25 & 0.20 \\ 26 & 0.20 & 27 & 0.20 & 28 & 0.20 & 29 & 0.20 & 30 & 0.20 \\ 31 & 0.20 & 32 & 0.20 & 33 & 0.20 & 34 & 0.20 & 35 & 0.20 \\ 36 & 0.20 & 37 & 0.20 & 38 & 0.20 & 39 & 0.20 & 40 & 0.20 \\ 41 & 0.20 & 42 & 0.20 & 43 & 0.20 & 44 & 0.20 & 45 & 0.20 \\ 46 & 0.20 & 47 & 0.20 & 48 & 0.20 & 49 & 0.20 & 50 & 0.20\end{array}$

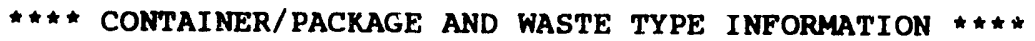

No. OF WASTE CONTA INERS/PACKAGES . . . . . . . 12

NO. OF CONTAINER/PACKAGE TYPES . . . . . . . . . . 1

TIME OF BREACH (YRS) FOR EACH CONTAINER

$\begin{array}{rrrrrrrr}\text { CONT. } & \text { TIME } & \text { CONT. } & \text { TIME } & \text { CONT. } & \text { TIME } & \text { CONT. } & \text { TIME } \\ 1 & 0.00 E+00 & 2 & 1.00 E+01 & 3 & 2.00 E+01 & 4 & 3.00 E+01 \\ 5 & 4.00 E+01 & 6 & 0.00 E+00 & 7 & 1.00 E+01 & 8 & 1.00 \mathrm{E}+01 \\ 9 & 2.00 E+01 & 10 & 2.00 \mathrm{E}+01 & 11 & 0.00 \mathrm{E}+00 & 12 & 3.00 \mathrm{E}+01\end{array}$




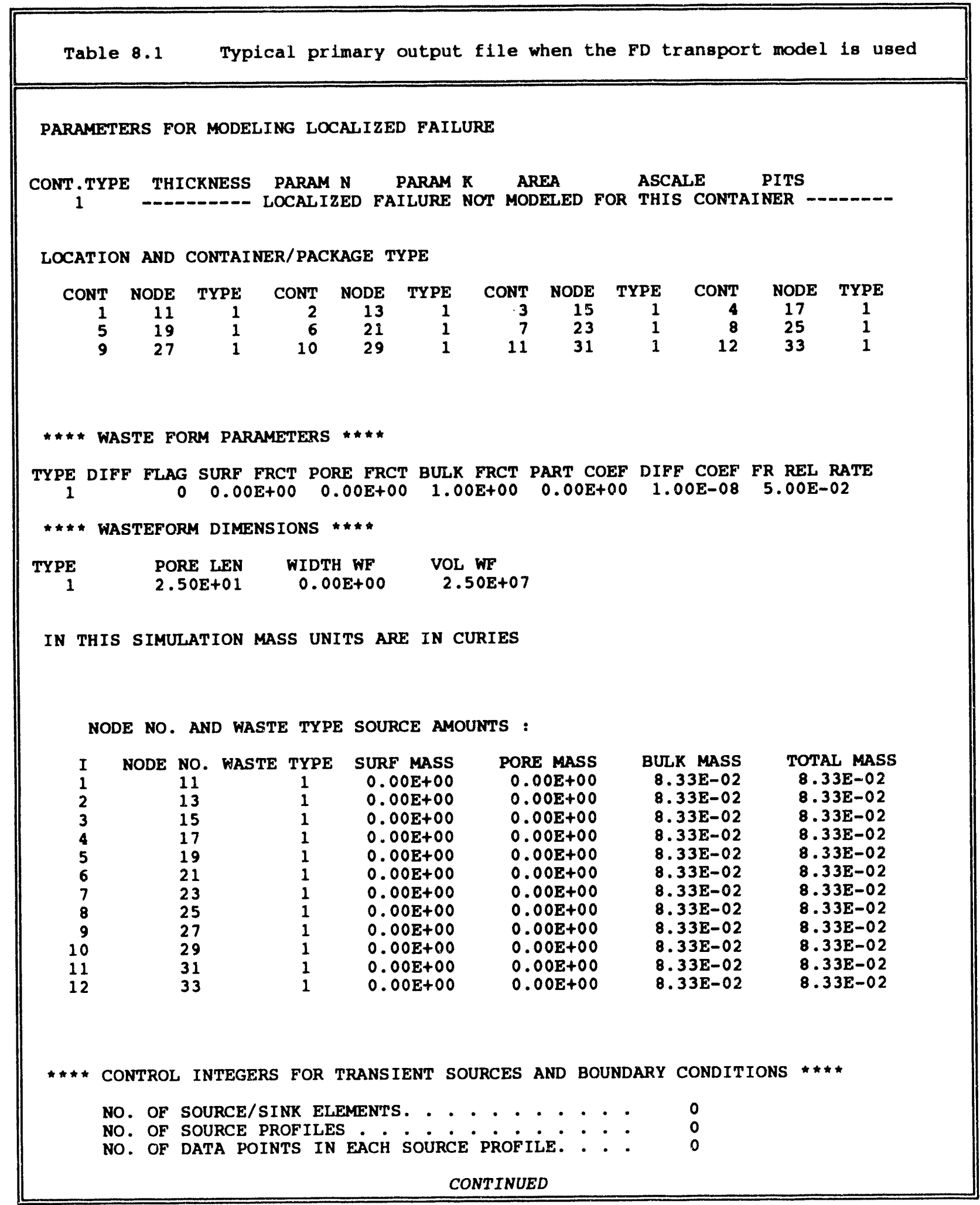


Table 8.1 Typical primary output file when the FD transport model is used

COMPLETION OF INPUT ROUTINE

BEGINNING OF CALCULATION

OUTPUT TABLE $1 \ldots$ CONCENTRATIONS AT TIME $=1.0000 E+00,($ DELT $=1.0000 E+00)$

$\star \star$ ITIME $=$

NODE I CONCENTRATION $\left(\mathrm{CI} / \mathrm{cm}^{\wedge} 3\right)$ AT NODES $\mathrm{I}, \mathrm{I}+1, \ldots, I+5$

$\begin{array}{rlllll}1 & 0.0000 \mathrm{E}+00 & 0.0000 \mathrm{E}+00 & 0.0000 \mathrm{E}+00 & 0.0000 \mathrm{E}+00 & 0.0000 \mathrm{E}+00 \\ 6 & 0.0000 \mathrm{E}+00 & 0.0000 \mathrm{E}+00 & 0.0000 \mathrm{E}+00 & 0.0000 \mathrm{E}+00 & 0.0000 \mathrm{E}+00 \\ 11 & 1.7118 \mathrm{E}-09 & 3.5691 \mathrm{E}-10 & 7.4417 \mathrm{E}-11 & 1.5516 \mathrm{E}-11 & 3.2352 \mathrm{E}-12 \\ 16 & 6.7454 \mathrm{E}-13 & 1.4064 \mathrm{E}-13 & 2.9324 \mathrm{E}-14 & 6.1142 \mathrm{E}-15 & 1.2748 \mathrm{E}-15 \\ 21 & 1.7118 \mathrm{E}-09 & 3.5691 \mathrm{E}-10 & 7.4417 \mathrm{E}-11 & 1.5516 \mathrm{E}-11 & 3.2352 \mathrm{E}-12 \\ 26 & 6.7454 \mathrm{E}-13 & 1.4064 \mathrm{E}-13 & 2.9324 \mathrm{E}-14 & 6.1142 \mathrm{E}-15 & 1.2748 \mathrm{E}-15 \\ 31 & 1.7118 \mathrm{E}-09 & 3.5691 \mathrm{E}-10 & 7.4417 \mathrm{E}-11 & 1.5516 \mathrm{E}-11 & 3.2352 \mathrm{E}-12 \\ 36 & 6.7454 \mathrm{E}-13 & 1.4064 \mathrm{E}-13 & 2.9324 \mathrm{E}-14 & 6.1142 \mathrm{E}-15 & 1.2748 \mathrm{E}-15 \\ 41 & 2.6580 \mathrm{E}-16 & 5.5421 \mathrm{E}-17 & 1.1555 \mathrm{E}-17 & 2.4093 \mathrm{E}-18 & 5.0235 \mathrm{E}-19 \\ 46 & 1.0474 \mathrm{E}-19 & 2.1839 \mathrm{E}-20 & 4.5534 \mathrm{E}-21 & 9.4939 \mathrm{E}-22 & 3.2760 \mathrm{E}-22\end{array}$

OUTPUT TABLE 2 Mass Flux at Time $=1.000 E+00$, Units are $\mathrm{Ci} / \mathrm{cm}^{\wedge} 2 / \mathrm{yr}$

$\star \star \star$ ITIME $=1$

\begin{tabular}{|c|c|c|c|c|c|c|c|}
\hline $\begin{array}{r}\text { NODE } \\
1 \\
5 \\
9 \\
13 \\
17 \\
21 \\
25 \\
29 \\
33 \\
37 \\
41 \\
45 \\
49\end{array}$ & $\begin{array}{r}\text { FLUX } \\
0.00 \mathrm{E}+00 \\
0.00 \mathrm{E}+00 \\
0.00 \mathrm{E}+00 \\
3.73 \mathrm{E}-09 \\
7.04 \mathrm{E}-12 \\
8.57 \mathrm{E}-08 \\
1.62 \mathrm{E}-10 \\
3.06 \mathrm{E}-13 \\
3.73 \mathrm{E}-09 \\
7.04 \mathrm{E}-12 \\
1.33 \mathrm{E}-14 \\
2.52 \mathrm{E}-17 \\
4.75 \mathrm{E}-20\end{array}$ & $\begin{array}{r}\text { NODE } \\
2 \\
6 \\
10 \\
14 \\
18 \\
22 \\
26 \\
30 \\
34 \\
38 \\
42 \\
46 \\
50\end{array}$ & $\begin{array}{r}\text { FLUX } \\
0.00 \mathrm{E}+00 \\
0.00 \mathrm{E}+00 \\
0.00 \mathrm{E}+00 \\
7.77 \mathrm{E}-10 \\
1.47 \mathrm{E}-12 \\
1.79 \mathrm{E}-08 \\
3.38 \mathrm{E}-11 \\
6.38 \mathrm{E}-14 \\
7.77 \mathrm{E}-10 \\
1.47 \mathrm{E}-12 \\
2.78 \mathrm{E}-15 \\
5.25 \mathrm{E}-18 \\
1.64 \mathrm{E}-20\end{array}$ & $\begin{array}{r}\text { NODE } \\
3 \\
7 \\
11 \\
15 \\
19 \\
23 \\
27 \\
31 \\
35 \\
39 \\
43 \\
47\end{array}$ & $\begin{array}{r}\text { FLUX } \\
0.00 \mathrm{E}+00 \\
0.00 \mathrm{E}+00 \\
8.57 \mathrm{E}-08 \\
1.62 \mathrm{E}-10 \\
3.06 \mathrm{E}-13 \\
3.73 \mathrm{E}-09 \\
7.04 \mathrm{E}-12 \\
8.57 \mathrm{E}-08 \\
1.62 \mathrm{E}-10 \\
3.06 \mathrm{E}-13 \\
5.79 \mathrm{E}-16 \\
1.09 \mathrm{E}-18\end{array}$ & $\begin{array}{r}\text { NODE } \\
4 \\
8 \\
12 \\
16 \\
20 \\
24 \\
28 \\
32 \\
36 \\
40 \\
44 \\
48\end{array}$ & $\begin{array}{r}\text { FLUX } \\
0.00 \mathrm{E}+00 \\
0.00 \mathrm{E}+00 \\
1.79 \mathrm{E}-08 \\
3.38 \mathrm{E}-11 \\
6.38 \mathrm{E}-14 \\
7.77 \mathrm{E}-10 \\
1.47 \mathrm{E}-12 \\
1.79 \mathrm{E}-08 \\
3.38 \mathrm{E}-11 \\
6.38 \mathrm{E}-14 \\
1.21 \mathrm{E}-16 \\
2.28 \mathrm{E}-19\end{array}$ \\
\hline
\end{tabular}


Table 8.1 Typical primary output file when the FD transport model is used

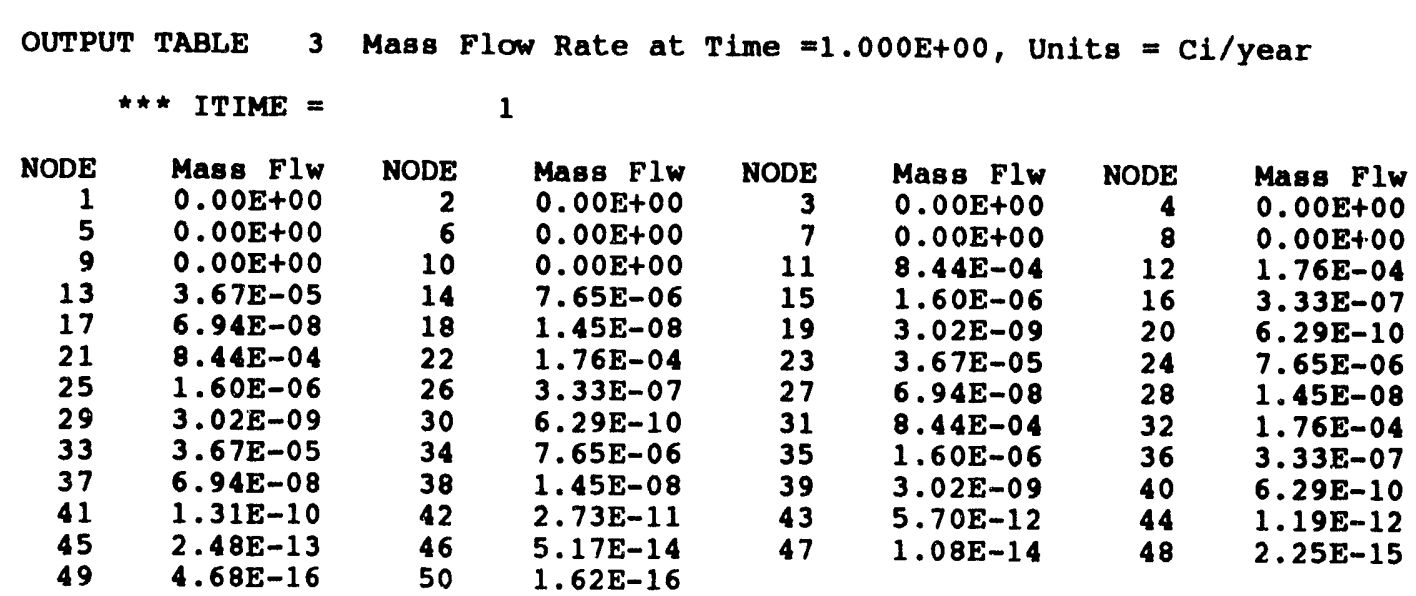

OUTPUT TABLE 3, WASTE FORM RELEASE DATA AT TIME $=1.0000 E+00$ $($ DELT $=1.00 \mathrm{E}+00) \quad \star \star \star$ ITIME $=1$

CONTAINER BREACH

LOCATION CONTAINER TIME BREACHED CONTAINER RATIO TYPE BREACH AREA AREA

$\begin{array}{llllll}11 & 1 & 0.00 \mathrm{E}+00 & 1.00 \mathrm{E}+00 & 1.00 \mathrm{E}+00 & 1.00 \mathrm{E}+00 \\ 21 & 1 & 0.00 \mathrm{E}+00 & 1.00 \mathrm{E}+00 & 1.00 \mathrm{E}+00 & 1.00 \mathrm{E}+00 \\ 31 & 1 & 0.00 \mathrm{E}+00 & 1.00 \mathrm{E}+00 & 1.00 \mathrm{E}+00 & 1.00 \mathrm{E}+00\end{array}$

SOURCE TERM TO THE TRANSPORT CALCULATION

IN $\mathrm{Ci} / \mathrm{CM}-\mathrm{CM}-\mathrm{CM}-\mathrm{S}$

LOCATION

CONTAINER WASTE

TYPE

TYPE

11
21
31

1
1
1

1
1

TOTAL
REL RATE

RINSE

REL RATE

$\begin{array}{ll}1.31 \mathrm{E}-16 & 0.00 \mathrm{E}+00 \\ 1.31 \mathrm{E}-16 & 0.00 \mathrm{E}+00 \\ 1.31 \mathrm{E}-16 & 0.00 \mathrm{E}+00\end{array}$

DIFFUSION REL RATE

$$
\begin{array}{ll}
0.00 \mathrm{E}+00 & 1.31 \mathrm{E}-16 \\
0.00 \mathrm{E}+00 & 1.31 \mathrm{E}-16 \\
0.00 \mathrm{E}+00 & 1.31 \mathrm{E}-16
\end{array}
$$

UNIFORM

REL RATE 


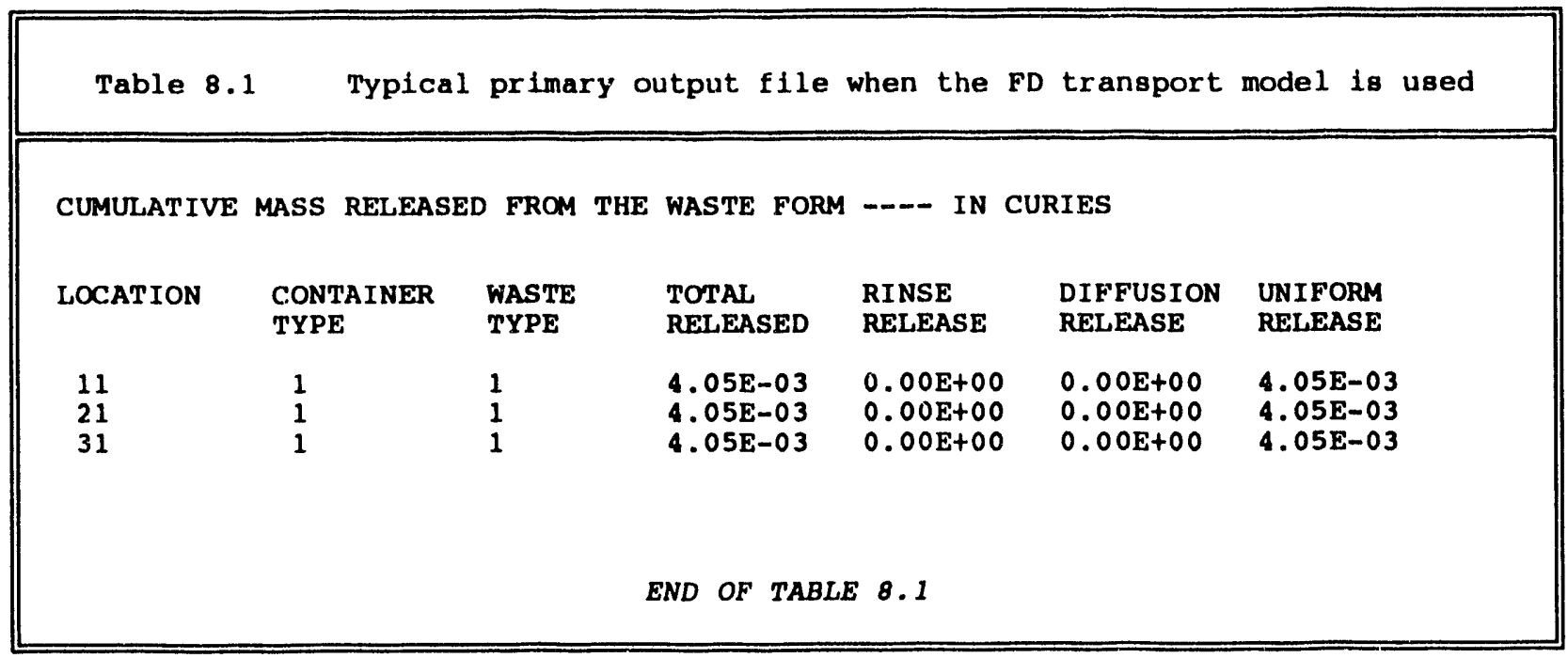


In Table 8.1 the first output from the calculation is the concentration at every point. These concentrations are also written to the file CONCNT.DAT which may be used by the graphics program GRAFXT. In the sample problem, containers in nodes 11, 21, and 31 all failed at the beginning of the problem, (Time $=0)$. From the concentrations we see that they are largest in these three nodes, as expected. Output for this time step would stop here if KPR $=1$.

Following the concentrations in Table 8.1 is the flux at each location. Again, highest fluxes occur at the source locations. Notice that the flux from node 11 is positive, i.e., flow is downstream only and there is no upstream dispersion. This is caused by the use of the upstream differencing and the use of zero values for dispersion and diffusion terms. This is the reason the concentration in nodes $1-10$ are zero. Output for this time step would stop here if KPR $=2$.

Following the fluxes, container breach information and wasteform release information is presented only for the containers that have failed. In this example, total failure occurs at time $=0$ for containers in nodes 11,21 , and 31 . For these containers, localized corrosion is not modeled, therefore, the container area and breached area are given default values of 1.0. If localized failure is modeled, the container and breached area would be printed. After the container information, the wasteform release rates and total mass released for all three containers are printed.

Further output is controlled by the value of KPR at each time step.

The output file for the MCMC model is slightly different once the calculation begins. In the MCMC model, the concentration, flux and mass flow rate are calculated only at the nodes specified through the use of the Trace Nodes. Further, since this model does not permit localized failure, all container failure times are known from the input. Also, wasteform release rates are known from input, either rinse release is specified, all released immediately after breach, or the fractional release rate is known. For these reasons, information on container failure time and wasteform release rate are not printed in the output.

If the output flag KPR is non-zero at a time step, and the MCMC model is specified, the location, concentration, flux, and mass flow rate at each requested computational point is printed. An example, is provided in Table 8.2, where nodes $1,7,12,23$, and 34 have been designated as trace nodes.

\subsection{Concentration Trace File (TRACECND.DAT)}

The concentration trace file is intended primarily for graphical output. It can be used directly by the program GRAFXT for visual output on the video screen as well as imported into graphics programs or spread sheets for hard copy output. It contains the concentrations at the requested trace locations as a function of time. 
Table 8.2 Typical primary output during the computation when the MCMC transport model is used

BEGINNING OF CALCULATION

OUTPUT TABLE 1, Concentration and Flux data at Time $=1.0000 \mathrm{E}+00$ Mass Units are Cí $i \star \star$ ITIME $=1$

MIXING BATH MODEL PREDICTED CONCENTRATIONS AND FLUXES

$\begin{array}{rlccc}\text { LOC } & \text { DIST ( } \mathrm{Cm}) & \text { CONC Cl/cm^3 } & \text { FLUX C1/cm^2/Yr } & \text { FLOW RATE C1/Yr } \\ 1 & 1.00 \mathrm{CH}+02 & 1.94 \mathrm{E}-09 & 9.71 \mathrm{E}-08 & 9.57 \mathrm{E}-04 \\ 7 & 7.00 \mathrm{E}+02 & 1.60 \mathrm{E}-16 & 8.03 \mathrm{E}-15 & 7.91 \mathrm{E}-11 \\ 12 & 1.20 \mathrm{E}+03 & 2.57 \mathrm{E}-10 & 1.29 \mathrm{E}-08 & 1.27 \mathrm{E}-04 \\ 23 & 2.30 \mathrm{E}+03 & 2.33 \mathrm{E}-11 & 1.17 \mathrm{E}-09 & 1.15 \mathrm{E}-05 \\ 34 & 3.40 \mathrm{E}+03 & 9.76 \mathrm{E}-25 & 4.89 \mathrm{E}-23 & 4.81 \mathrm{E}-19\end{array}$

OUTPUT TABLE 2, Concentration and Flux data at Time $=2.0000 \mathrm{E}+00$ Mass Units are Ci $i * \star$ ITIME $=2$

MIXING BATH MODEL PREDICTED CONCENTRATIONS AND FLUXES

$\begin{array}{rcccc}\text { LOC } & \text { DIST }(\mathrm{cm}) & \text { CONC } \mathrm{CI} / \mathrm{cm}^{\wedge} 3 & \text { FLUX C1/cm^2/Yr } & \text { FLOW RATE C1/Yr } \\ 1 & 1.00 \mathrm{CH}+02 & 3.22 \mathrm{E}-09 & 1.61 \mathrm{E}-07 & 1.59 \mathrm{E}-03 \\ 7 & 7.00 \mathrm{E}+02 & 1.52 \mathrm{E}-14 & 7.63 \mathrm{E}-13 & 7.52 \mathrm{E}-09 \\ 12 & 1.20 \mathrm{E}+03 & 8.14 \mathrm{E}-10 & 4.07 \mathrm{E}-08 & 4.01 \mathrm{E}-04 \\ 23 & 2.30 \mathrm{E}+03 & 1.44 \mathrm{E}-10 & 7.20 \mathrm{E}-09 & 7.09 \mathrm{E}-05 \\ 34 & 3.40 \mathrm{E}+03 & 1.37 \mathrm{E}-23 & 6.84 \mathrm{E}-22 & 6.74 \mathrm{E}-18\end{array}$

OUTPUT TABLE 3, Concentration and Flux data at Time $=5.0000 \mathrm{E}+00$ Mass Units are $C i \quad$ ITIME $=3$

MIXING BATH MODEL PREDICTED CONCENTRATIONS AND FLUXES

\begin{tabular}{|c|c|c|c|c|}
\hline $\begin{array}{r}\text { LOC } \\
1 \\
7 \\
12 \\
23 \\
34\end{array}$ & $\begin{array}{l}\text { DIST (CnI) } \\
1.00 \mathrm{E}+02 \\
7.00 \mathrm{E}+02 \\
1.20 \mathrm{E}+03 \\
2.30 \mathrm{E}+03 \\
3.40 \mathrm{E}+03\end{array}$ & $\begin{array}{c}\operatorname{CONC} \mathrm{CL}_{1 / \mathrm{cm}^{\wedge} 3} \\
4.79 \mathrm{E}-09 \\
3.83 \mathrm{E}-12 \\
2.58 \mathrm{E}-09 \\
1.05 \mathrm{E}-09 \\
2.04 \mathrm{E}-18\end{array}$ & $\begin{array}{c}\text { FLUX } \mathrm{Ci} / \mathrm{cm}^{\wedge} 2 / \mathrm{yr} \\
2.40 \mathrm{E}-07 \\
1.92 \mathrm{E}-10 \\
1.29 \mathrm{E}-07 \\
5.25 \mathrm{E}-08 \\
1.02 \mathrm{E}-16\end{array}$ & $\begin{array}{c}\text { FLOW RATE CI/YS } \\
2.36 \mathrm{E}-03 \\
1.89 \mathrm{E}-06 \\
1.27 \mathrm{E}-03 \\
5.17 \mathrm{E}-04 \\
1.01 \mathrm{E}-12\end{array}$ \\
\hline
\end{tabular}


At the top of this file are a number of cards which are used to define the problem. The format used on these cards is mandatory if the program GRAFXT is to be used. The first card in this file prints the title as defined by input. This is followed by a card providing the number of concentration traces and the units of concentration. This is followed by a blank card. Next is the list of the node numbers at which a trace is requested. These values are printed in columns such that the data underneath this label is the concentration at the specified node. The location of these nodes is printed on the next line. The last problem definition card prints the label TIME (YRS) over the first column of data and provides headings for the columns of concentration values.

The remainder of this file contains the time followed by the concentration at each of the trace locations. Up to 20 concentrations, the maximum number allowed by DUST, will be written on a single line. An example of a concentration trace file is presented in Table 8.3. The structure of this file is independent of the choice of transport inodel.

The file name TRACECND.DAT is used every time a trace file is requested by the DUST code. If the output of this file needs to be saved, the file must be renimed prior to using the DUST code.

\subsection{Flux Trace File (TRACEFXD.DAT)}

The flux trace file is also intended primarily for use with GRAFXT and other graphics programs. TRACEFXD contains the flux (units are mass $/ \mathrm{cm}^{2} / y r$ with the mass in either grams or Curies depending on mass units specified through input), an estimate for the cumulative amount of mass that has passed the location as a function of time (flux integrated over time and area, units of mass), and the mass flow rate (flux integrated over the area, units of mass/yr).

The structure of the file is similar to TRACECND, however, because it contains three variables per trace location, there are important differences. The first card is the title card. The next card provides the definition of the number of trace locations and the units used for the output. This is followed by a blank card.

At this point, the structure differs from that used in TRACECND. The values for the node are printed three times, once for the flux, the integrated flux, and for the mass flow rate. Up to 7 nodes, 21 values, are printed per line. If more than 7 flux trace nodes are specified, additional lines are printed. GRAFXT automatically adjusts for this. However, if TRACEFXD is imported into a spreadsheet or other graphics program, adjustments to the file may be required.

Following the node numbers, the location of each node is printed. Again, the location is printed three times, once for each variable of interest. After the locations are printed, the label TIME (YRS) is printed on a separate line and labels (flux, Mass Rel for the mass release, and Mass Rate for the mass flow rate) are printed for each column. 
Table 8.3 Typical concentration trace file, TRACECND.DAT

FD MODEL $-\mathrm{H}-3, M C=0.2$, VDAR=1.58E-6, MLT CONT

NUMBER OF CONCENTRATION TRACES $=5$ UnIts are Curies per cubic centimeter

\begin{tabular}{|c|c|c|c|c|c|}
\hline $\begin{array}{l}\text { NODE } \\
\text { DISTANCE } \\
\text { TIME (YRS) } \\
0.00 \mathrm{E}+00 \\
2.00 \mathrm{E}+00 \\
4.00 \mathrm{E}+00 \\
6.00 \mathrm{E}+00 \\
8.00 \mathrm{E}+00 \\
1.00 \mathrm{E}+01 \\
1.20 \mathrm{E}+01 \\
1.40 \mathrm{E}+01 \\
1.60 \mathrm{E}+01 \\
1.80 \mathrm{E}+01 \\
2.00 \mathrm{E}+01 \\
2.20 \mathrm{E}+01 \\
2.40 \mathrm{E}+01 \\
2.60 \mathrm{E}+01 \\
2.80 \mathrm{E}+01 \\
3.00 \mathrm{E}+01 \\
3.20 \mathrm{E}+01 \\
3.40 \mathrm{E}+01 \\
3.60 \mathrm{E}+01 \\
3.80 \mathrm{E}+01 \\
4.00 \mathrm{E}+01 \\
4.20 \mathrm{E}+01 \\
4.40 \mathrm{E}+01 \\
4.60 \mathrm{E}+01 \\
4.80 \mathrm{E}+01 \\
5.00 \mathrm{E}+01 \\
5.20 \mathrm{E}+01 \\
5.40 \mathrm{E}+01 \\
5.60 \mathrm{E}+01 \\
5.80 \mathrm{E}+01 \\
6.00 \mathrm{E}+01 \\
6.20 \mathrm{E}+01 \\
6.40 \mathrm{E}+01 \\
6.60 \mathrm{E}+01 \\
6.80 \mathrm{E}+01 \\
7.00 \mathrm{E}+01 \\
7.20 \mathrm{E}+01 \\
7.40 \mathrm{E}+01 \\
7.60 \mathrm{E}+01 \\
7.80 \mathrm{E}+01 \\
8.00 \mathrm{E}+01 \\
8.20 \mathrm{E}+01 \\
8.40 \mathrm{E}+01 \\
8.60 \mathrm{E}+01 \\
8.80 \mathrm{E}+01 \\
9.00 \mathrm{E}+01 \\
\end{array}$ & $\begin{array}{c}11 \\
1.00 \mathrm{E}+03 \\
\text { Conc } \\
0.00 \mathrm{E}+00 \\
2.90 \mathrm{E}-09 \\
4.22 \mathrm{E}-09 \\
4.69 \mathrm{E}-09 \\
4.70 \mathrm{E}-09 \\
4.49 \mathrm{E}-09 \\
4.18 \mathrm{E}-09 \\
3.82 \mathrm{E}-09 \\
3.47 \mathrm{E}-09 \\
3.13 \mathrm{E}-09 \\
2.81 \mathrm{E}-09 \\
1.58 \mathrm{E}-09 \\
8.87 \mathrm{E}-10 \\
4.98 \mathrm{E}-10 \\
2.80 \mathrm{E}-10 \\
1.57 \mathrm{E}-10 \\
8.82 \mathrm{E}-11 \\
4.95 \mathrm{E}-11 \\
2.78 \mathrm{E}-11 \\
1.56 \mathrm{E}-11 \\
8.77 \mathrm{E}-12 \\
4.92 \mathrm{E}-12 \\
2.76 \mathrm{E}-12 \\
1.55 \mathrm{E}-12 \\
8.72 \mathrm{E}-13 \\
4.90 \mathrm{E}-13 \\
2.75 \mathrm{E}-13 \\
1.54 \mathrm{E}-13 \\
8.67 \mathrm{E}-14 \\
4.87 \mathrm{E}-14 \\
2.73 \mathrm{E}-14 \\
1.54 \mathrm{E}-14 \\
8.62 \mathrm{E}-15 \\
4.84 \mathrm{E}-15 \\
2.72 \mathrm{E}-15 \\
1.53 \mathrm{E}-15 \\
8.57 \mathrm{E}-16 \\
4.81 \mathrm{E}-16 \\
2.70 \mathrm{E}-16 \\
1.52 \mathrm{E}-16 \\
8.53 \mathrm{E}-17 \\
4.79 \mathrm{E}-17 \\
2.69 \mathrm{E}-17 \\
1.51 \mathrm{E}-17 \\
8.48 \mathrm{E}-18\end{array}$ & $\begin{array}{c}17 \\
1.60 \mathrm{E}+03 \\
\text { Conc } \\
0.00 \mathrm{E}+00 \\
8.71 \mathrm{E}-13 \\
7.84 \mathrm{E}-12 \\
3.12 \mathrm{E}-11 \\
8.17 \mathrm{E}-11 \\
1.65 \mathrm{E}-10 \\
2.89 \mathrm{E}-10 \\
4.67 \mathrm{E}-10 \\
7.02 \mathrm{E}-10 \\
9.77 \mathrm{E}-10 \\
1.26 \mathrm{E}-09 \\
1.60 \mathrm{E}-09 \\
1.98 \mathrm{E}-09 \\
2.33 \mathrm{E}-09 \\
2.59 \mathrm{E}-09 \\
2.74 \mathrm{E}-09 \\
3.30 \mathrm{E}-09 \\
3.47 \mathrm{E}-09 \\
3.39 \mathrm{E}-09 \\
3.17 \mathrm{E}-09 \\
2.87 \mathrm{E}-09 \\
2.52 \mathrm{E}-09 \\
2.15 \mathrm{E}-09 \\
1.78 \mathrm{E}-09 \\
1.45 \mathrm{E}-09 \\
1.17 \mathrm{E}-09 \\
7.71 \mathrm{E}-10 \\
5.09 \mathrm{E}-10 \\
3.36 \mathrm{E}-10 \\
2.23 \mathrm{E}-10 \\
1.47 \mathrm{E}-10 \\
9.74 \mathrm{E}-11 \\
6.43 \mathrm{E}-11 \\
4.23 \mathrm{E}-11 \\
2.78 \mathrm{E}-11 \\
1.82 \mathrm{E}-11 \\
1.19 \mathrm{E}-11 \\
7.78 \mathrm{E}-12 \\
5.06 \mathrm{E}-12 \\
3.28 \mathrm{E}-12 \\
2.12 \mathrm{E}-12 \\
1.37 \mathrm{E}-12 \\
8.82 \mathrm{E}-13\end{array}$ & $\begin{array}{l}22 \\
2.10 \mathrm{E}+03 \\
\text { ConC } \\
0.00 \mathrm{E}+00 \\
8.73 \mathrm{E}-10 \\
1.95 \mathrm{E}-09 \\
2.78 \mathrm{E}-09 \\
3.28 \mathrm{E}-09 \\
3.50 \mathrm{E}-09 \\
3.52 \mathrm{E}-09 \\
3.41 \mathrm{E}-09 \\
3.22 \mathrm{E}-09 \\
3.00 \mathrm{E}-09 \\
2.77 \mathrm{E}-09 \\
2.27 \mathrm{E}-09 \\
1.73 \mathrm{E}-09 \\
1.30 \mathrm{E}-09 \\
9.98 \mathrm{E}-10 \\
8.22 \mathrm{E}-10 \\
7.40 \mathrm{E}-10 \\
7.26 \mathrm{E}-10 \\
7.59 \mathrm{E}-10 \\
8.21 \mathrm{E}-10 \\
8.95 \mathrm{E}-10 \\
9.75 \mathrm{E}-10 \\
1.06 \mathrm{E}-09 \\
1.13 \mathrm{E}-09 \\
1.19 \mathrm{E}-09 \\
1.22 \mathrm{E}-09 \\
1.22 \mathrm{E}-09 \\
1.18 \mathrm{E}-09 \\
1.12 \mathrm{E}-09 \\
1.04 \mathrm{E}-09 \\
9.41 \mathrm{E}-10 \\
8.32 \mathrm{E}-10 \\
7.17 \mathrm{E}-10 \\
6.03 \mathrm{E}-10 \\
4.94 \mathrm{E}-10 \\
3.95 \mathrm{E}-10 \\
3.10 \mathrm{E}-10 \\
2.39 \mathrm{E}-10 \\
1.81 \mathrm{E}-10 \\
1.35 \mathrm{E}-10 \\
9.97 \mathrm{E}-11 \\
7.28 \mathrm{E}-11 \\
5.27 \mathrm{E}-11 \\
3.78 \mathrm{E}-11 \\
2.69 \mathrm{E}\end{array}$ & $\begin{array}{c}33 \\
3.20 \mathrm{E}+03 \\
\text { Conc } \\
0.00 \mathrm{E}+00 \\
2.38 \mathrm{E}-10 \\
7.63 \mathrm{E}-10 \\
1.38 \mathrm{E}-09 \\
1.94 \mathrm{E}-09 \\
2.36 \mathrm{E}-09 \\
2.61 \mathrm{E}-09 \\
2.72 \mathrm{E}-09 \\
2.72 \mathrm{E}-09 \\
2.64 \mathrm{E}-09 \\
2.53 \mathrm{E}-09 \\
2.32 \mathrm{E}-09\end{array}$ & $\begin{array}{l}44 \\
4.30 \mathrm{E}+03 \\
\text { Conc } \\
0.00 \mathrm{E}+00 \\
2.76 \mathrm{E}-17 \\
7.27 \mathrm{E}-16 \\
7.24 \mathrm{E}-15 \\
4.22 \mathrm{E}-14 \\
1.73 \mathrm{E}-13 \\
5.52 \mathrm{E}-13 \\
1.45 \mathrm{E}-12 \\
3.30 \mathrm{E}-12 \\
6.61 \mathrm{E}-12 \\
1.20 \mathrm{E}-11 \\
2.00 \mathrm{E}-11 \\
3.11 \mathrm{E}-11 \\
4.54 \mathrm{E}-11 \\
6.28 \mathrm{E}-11 \\
8.29 \mathrm{E}-11 \\
1.05 \mathrm{E}-10 \\
1.28 \mathrm{E}-10 \\
1.51 \mathrm{E}-10 \\
1.73 \mathrm{E}-10 \\
1.93 \mathrm{E}-10 \\
2.11 \mathrm{E}-10 \\
2.26 \mathrm{E}-10 \\
2.38 \mathrm{E}-10 \\
2.48 \mathrm{E}-10 \\
2.56 \mathrm{E}-10 \\
2.63 \mathrm{E}-10 \\
2.68 \mathrm{E}-10 \\
2.74 \mathrm{E}-10 \\
2.78 \mathrm{E}-10 \\
2.83 \mathrm{E}-10 \\
2.86 \mathrm{E}-10 \\
2.89 \mathrm{E}-10 \\
2.91 \mathrm{E}-10 \\
2.91 \mathrm{E}-10 \\
2.88 \mathrm{E}-10 \\
2.84 \mathrm{E}-10 \\
2.77 \mathrm{E}-10 \\
2.67 \mathrm{E}-10 \\
2.56 \mathrm{E}-10 \\
2.42 \mathrm{E}-10 \\
2.27 \mathrm{E}-10 \\
2.11 \mathrm{E}-10 \\
1.94 \mathrm{E}-10 \\
1.78 \mathrm{E}-10 \\
1.61 \mathrm{E}-10\end{array}$ \\
\hline
\end{tabular}


The remainder of the file contains a series of columns of values. The first column is the time, the second column is the flux at the first trace location, the third column is the integrated flux (units of mass, either Curies or grams depending on the input), and the fourth column is the mass flow rate (units of mass per year with the mass units in Curies or grams depending on the input). The three values (flux, integrated flux, and mass flow rate), are repeated for each trace location. If more than 7 trace locations are specified, additional cards are needed to define the output at each time. An example of a flux trace file is presented in Table 8.4. Although, the example is for the FD transport model, the structure is identical if the MCMC model was used.

The file name TRACEFXD.DAT is used every time a trace file is requested by the DUST code. If the output of this file needs to be saved, the file must be renamed prior to reuse of the DUST code.

\subsection{Concentration File (CONCNT.DAT)}

When the FD transport model is used, every time the print flag KPR is greater than zero, the concentration at every location is written to the primary output file and to CONCNT.DAT. The file CONCNT.DAT complements the file TRACECND.DAT. Typically, CONCNT.DAT will have the concentrations at every location at a few specified times while TRACECND.DAT will have the concentrations at a few locations at almost every time step.

The first line in CONCNT.DAT contains the title specified by the code user. The second line defines the number of locations and the units for concentration in the output file. These are followed by the value for the locations beginning with the first node. In CONCNT.DAT, since the number of locations will vary between different problems, the WRITE statements print out seven values per line. The number of lines printed depends on the number of values. In the example provided in Table 8.5, there are 50 locations. Therefore, there are 8 lines containing locations (seven with seven values and the last with one value). After the locations are printed, a line containing the problem time at which the concentration values were written is displayed. This is followed by the values for the concentration at each node. Again, these are written seven to a line beginning with Node 1 .

Each time output is requested, the sequence of lines containing the time followed by the concentration values is repeated, Table 8.5 .

As this file stands, it is not in a form that is useful for importing into spreadsheets or other graphics packages. To overcome this limitation, the program IOSWTCH was written. IOSWTCH reads and reformats the file CONCNT.DAT. It creates a new file CONCNT2.DAT that contains the title card followed by a card specifying the number of locations. This is followed by a series of rows and columns of primarily numerical data. The first row contains the alphanumeric string "TIME/LOC" in column 1 while columns 2 through the end contain the output time. The second row contains the location in column 1 and the values for concentration at the various time in columns 2 through the end. That is, the output file can be viewed as a 
Table 8.4 Typlcal flux trace flle, TRACEFXD.DAT

FD MODEL $-H-3, M C \equiv 0.2, V D A R=1.58 E-6, M L T$ CONT

NUMBER OF FLUX TRACES $=5$ Flux units are Curles per $\mathrm{cm}^{\wedge} 2 / \mathrm{yr}$

$\begin{array}{lc}\text { NODE } & 11 \\ \text { DISTANCE } & 1.00 \mathrm{E}+03\end{array}$

TIME (YRS) FluX

$0.00 \mathrm{E}+00 \quad 0.00 \mathrm{E}+00$

$2.00 \mathrm{E}+00 \quad 1.45 \mathrm{E}-07$

4.00E+00 2.12E-07

$6.00 E+00 \quad 2.35 E-07$

8. $00 \mathrm{E}+00 \quad 2.36 \mathrm{E}-07$

$1.00 \mathrm{E}+01$

$1.20 E+01$

1. $40 \mathrm{E}+01$

$1.60 \mathrm{E}+01$

$1.80 E+01$

2. $00 \mathrm{E}+01$

$2.20 E+01$

2. $40 E+01$

2. 60E+01

2. $80 \mathrm{E}+01$

3. $00 \mathrm{E}+01$

3. $20 E+01$

3. $40 \mathrm{E}+01$

$3.60 \mathrm{E}+01$

$3.80 \mathrm{E}+01$

4.00E+01

4. $20 E+01$

4. $40 \mathrm{E}+01$

$4.60 \mathrm{E}+01$

4. $80 E+01$

5.00E+01

5. $20 E+01$

5. $40 \mathrm{E}+01$

5.60E+01

5.80E+01

6. $00 \mathrm{E}+01$

$6.20 \mathrm{E}+01$

$6.40 \mathrm{E}+01$

$6.60 \mathrm{E}+01$

$6.80 \mathrm{E}+01$

$7.00 E+01$

7. 20E+01

$7.40 \mathrm{E}+01$

$7.60 \mathrm{E}+01$

$7.80 \mathrm{E}+01$

8. $00 \mathrm{E}+01$

8. $20 \mathrm{E}+01$

8. $40 \mathrm{E}+01$

8. $60 \mathrm{E}+01$

$8.80 E+01$

9. $00 \mathrm{E}+01$
$2.36 E-07$
$2.25 E-07$

2.09E-07

1.92E-07

1.74E-07

1.57E-07

1. 41E-07

7.91E-08

4. 44E-08

$2.49 \mathrm{E}-08$

$1.40 \mathrm{E}-08$

$7.86 \mathrm{E}-09$

2.48E-09

1. 39E-09

7.82E-10

4.39E-10

2. $47 \mathrm{E}-10$

1. $38 \mathrm{E}-10$

7. $78 \mathrm{E}-11$

4.37E-11

2. 45E-11

1. $38 \mathrm{E}-11$

7. $73 \mathrm{E}-12$

4. 34E-12

2. 44E-12

1. 37E-12

7. $69 \mathrm{E}-13$

4. $32 \mathrm{E}-13$

$2.42 E-13$

1. 36E-13

7. 65E-14

4. 29E-14

2.41E-14

1. $35 \mathrm{E}-14$

7. $60 \mathrm{E}-15$

$4.27 \mathrm{E}-15$

2. $40 \mathrm{E}-15$

1. $35 \mathrm{E}-15$

$7.56 \mathrm{E}-16$

4. 25E-16

2. 38E-16
$4.42 \mathrm{E}-09$

\begin{abstract}
11
\end{abstract}
1. $00 \mathrm{OE}+03$

Masg Rel

$0.00 \mathrm{E}+00$

$2.28 E-03$

6.19E-03

$1.07 \mathrm{E}-02$

$1.54 \mathrm{E}-02$

1. $99 \mathrm{E}-02$

2.41E-02

2.80E-02

3. 15E-02

3. 46E-02

$3.75 \mathrm{E}-02$

3. $93 \mathrm{E}-02$

4. 03E-02

4.09E-02

$4.12 \mathrm{E}-02$

$4.14 E-02$

4.15E-02

$4.16 \mathrm{E}-02$

4.16E-02

4.16E-02

4.16E-02

4.16E-02

$4.16 \mathrm{E}-02$

4.16E-02

$4.16 \mathrm{E}-02$

$4.16 \mathrm{E}-02$

4.16E-02

$4.16 \mathrm{E}-02$

4.16E-02

$4.16 \mathrm{E}-02$

4.16E-02

$4.16 \mathrm{E}-02$

4. 16E-02

$4.16 \mathrm{E}-02$

$4.16 \mathrm{E}-02$

4.16E-02

4.16E-02

4.16E-02

$4.16 \mathrm{E}-02$

$4.16 \mathrm{E}-02$

4.16E-02

4. 16E-02

4.16E-02

4.16E-02

4.16E-02

4.16E-02
11

$1.00 E+03 \quad 1.60 E+03$

Mass Rate Flux

$0.00 \mathrm{E}+00 \quad 0.00 \mathrm{E}+00$

$1.43 E-03$

2.08E-03

2. 31E-03

2.32E-03

$2.22 \mathrm{E}-03$

2.06E-03

1.89E-03

$1.71 \mathrm{E}-03$

$1.54 \mathrm{E}-03$

$1.39 E-03$

7.79E-04

$4.37 \mathrm{E}-04$

2.46E-04

$1.38 \mathrm{E}-04$

$7.75 E-05$

4. 35E-05

2. 44E-05

1.37E-05

7. $70 \mathrm{E}-06$

4. 33E-06

$2.43 \mathrm{E}-06$

1. 36E-06

7. $66 \mathrm{E}-07$

4. 30E-07

2.42E-07

1. 36E-07

7. $62 \mathrm{E}-08$

4. 28E-08

2. $40 \mathrm{E}-08$

1. 35E-08

$7.57 \mathrm{E}-09$

4. 25E-09

2.39E-09

1.34E-09

7. $53 \mathrm{E}-10$

4. $23 E-10$

2. 38E-10

1. $33 E-10$

7. $49 \mathrm{E}-11$

4. 21E-11

2. 36E-11

1. $33 E-11$

7. $45 E-12$

4. 18E-12

2. $35 E-12$
$4.36 \mathrm{E}-11$

3. $93 \mathrm{E}-10$

1. $56 \mathrm{E}-09$

4. 09E-09

$8.27 \mathrm{E}-09$

1. $45 \mathrm{E}-08$

2.34E-08

3. $52 \mathrm{E}-08$

$4.89 \mathrm{E}-08$

$6.31 \mathrm{E}-08$

8.01E-08

9.93E-08

$1.17 \mathrm{E}-07$

1.30E-07

$1.37 \mathrm{E}-07$

1. $65 \mathrm{E}-07$

$1.74 E-07$

1.70E-07

$1.59 \mathrm{E}-07$

$1.44 \mathrm{E}-07$

1.26E-07

$1.08 \mathrm{E}-07$

8. $92 \mathrm{E}-08$

$7.28 \mathrm{E}-08$

5. $88 \mathrm{E}-08$

3.86E-08

2. 55E-08

4. 88E-09

3. 22E-09

2.12E-09

1. 39E-09

9. $14 \mathrm{E}-10$

5. $98 E-10$

3. $90 \mathrm{E}-10$

2. $53 E-10$

1. $64 \mathrm{E}-10$

$1.06 \mathrm{E}-10$

6. $86 \mathrm{E}-11$

$4.42 E-11$

2. 83E-11

1. 82E-11

1. $16 E-11$
1. $68 \mathrm{E}-08$

$1.11 E-08$

7. $38 \mathrm{E}-09$
17

$1.60 \mathrm{E}+03$

Mass Rel

$0.00 \mathrm{E}+00$

$4.99 \mathrm{E}-07$

$5.87 \mathrm{E}-06$

2.95E-05

. $57 E-05$

$2.36 \mathrm{E}-04$

$4.87 \mathrm{E}-04$

$9.00 \mathrm{E}-04$

$1.53 E-03$

$2.43 \mathrm{E}-03$

$3.60 \mathrm{E}-03$

5.09E-03

6.95E-03

$9.17 \mathrm{E}-03$

$1.17 \mathrm{E}-02$

$1.43 \mathrm{E}-02$

1. $75 \mathrm{E}-02$

$2.09 \mathrm{E}-02$

$2.43 E-02$

2.75E-02

3. $04 \mathrm{E}-02$

3. $29 \mathrm{E}-02$

$3.51 \mathrm{E}-02$

3. $70 \mathrm{E}-02$

$3.85 \mathrm{E}-02$

$3.97 \mathrm{E}-02$

4. 06E-02

4.11E-02

$4.15 \mathrm{E}-02$

$4.18 \mathrm{E}-02$

4.19E-02

4. 20E-02

4.21E-02

$4.21 E-02$

4.22E-02

4.22E-02

$4.22 \mathrm{E}-02$

4.22E-02

4. 22E-02

4.22E-02

4. 22E-02

4. 22E-02

$4.22 E-02$

4.22E-02

4. 22E-02

4. 22E-02 


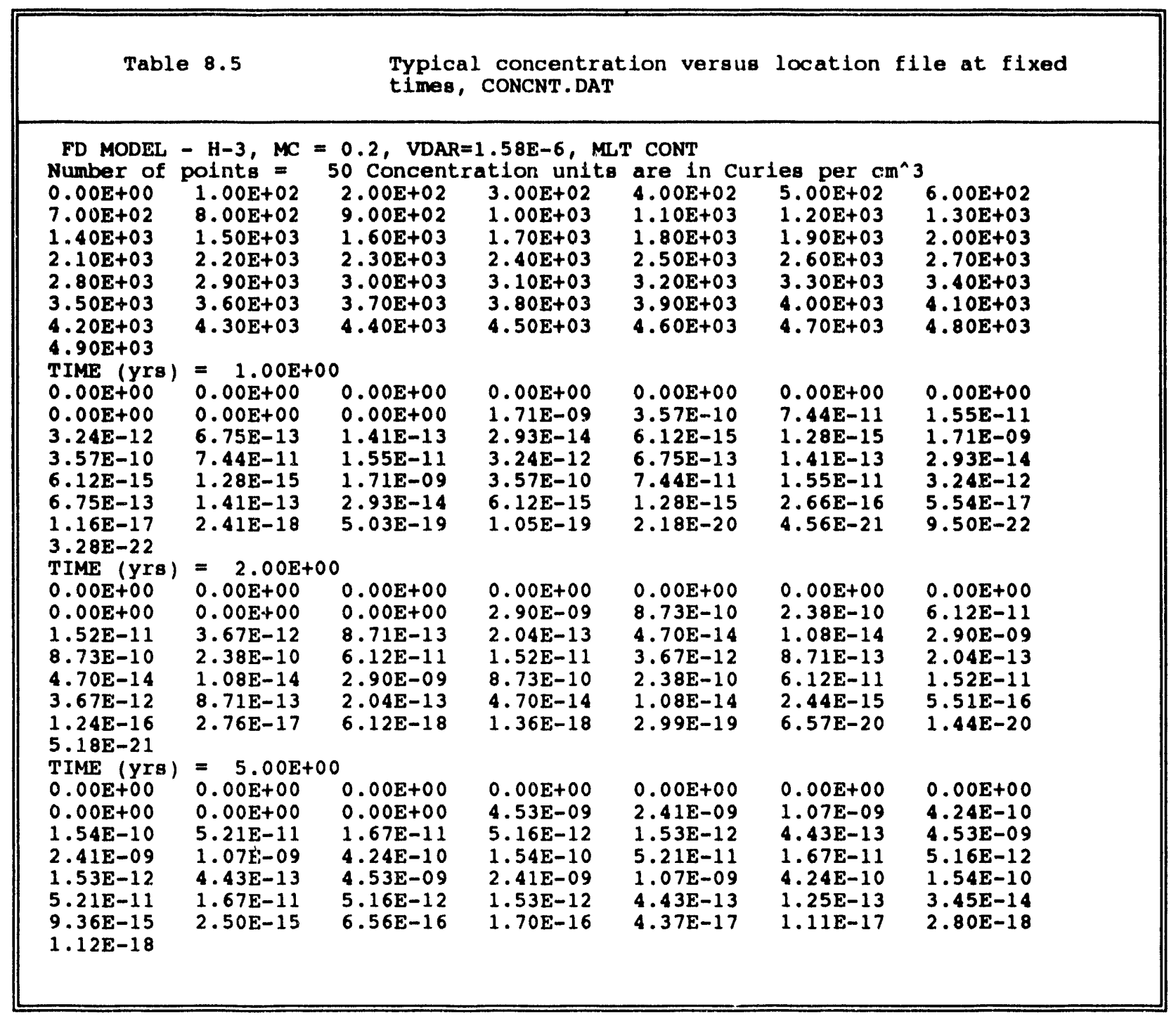


matrix containing columns of concentration versus location at fixed times (or alternatively, rows of concentration versus time at fixed locations).

Both CONCNT.DAT and CONCNT2.DAT are default file names which are overwritten by the programs that create them. Therefure, if the user desires to save the output, these files need to be renamed.

\subsection{Wasteform Release Data (LEACHRL.DAT)}

If the FD transport model is used, the file LEACHRL.DAT is written at every time step that a trace is performed. This file contains the total mass released by release mechanism (rinse, diffusion, or uniform) and the release rate for each mechanism. The ratio of the breached area to total area as well as the location of the wasteform are also printed. The fiie contains three label cards. The first identifies this file as the wasteform release file. The second and third label cards provide information to identify the columns, Table 8.6.

This file contains the following 11 columns:

\section{Columr:}

1) Time (yrs), the problem time at which the data are writien.

2) Cumulative, cumulative mass release from all mechanisms for this wasteform.

3) Rinse, cumulative mass release from the rinse model.

4) Diffusion, cumulative mass release from the diffusion model.

5) Uniform, cumulative mass release from the uniform release model.

6) Totai, total release rate from all mechanisms.

7) Rinse, rinse release rate.

8) Diffusion, diffusion release rate.

9) Uniform, uniform release rate.

10) Breach Ratio, tatio of breached container area to total area. A value of 1 indicates total failure of the container.

11) Node, location of the wasteform.

Columns 1-11 are written once for each wasteform at each time step. This file often becomes quitt large when information is written at every time step.

This file is written every time the DUST code uses the FD transport model. If this information needs to be saved, the file must be renamed upon completion of DUST.

The example in Table 8.6 is from the test problem discussed in Section 8.1. After three years, only containers in control volumes 11,21 , and 31 have failed. All other containers are unbreached and therefore have zero release. Also, the only release mechanism modeled is uniform release. 
Table 8.6

Typical wasteform release output file, LEACHRL.DAT

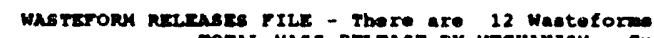

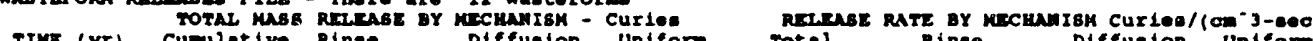

rotel pinse Diffueion Unifor

Broh Ratio Hodo

$2.00 \quad 7.0028-03$

$0.000 \Sigma+00$

$0.000 z+00$

$1.2325-26$

$0.000 z+00$

$0.000 z+00$

$1.2325-16$

$1.0005+00$

$2.00 \quad 0.0005+00 \quad 0.000 z+00 \quad 0.000 E+00 \quad 0.000 z+00$

$2.00 \quad 0.000 x+00 \quad 0.0005+00 \quad 0.0005+00$

$2.00 \quad 0.0005+00 \quad 0.0005+00$

$0.000 x+00 \quad 0.000 x+00$

$0.0005+00$

$0.0002+00 \quad 0.000 E+00$

$0.000 z+00 \quad 0.000 E+00$

$0.000 \mathrm{z}+00 \quad 0.000 \mathrm{~s}+00$

$0.0005+00$

$\begin{array}{lll}0.000 \Omega & 0.000 & 0.000 z+00\end{array}$

$\begin{array}{lll}7.8825-03 & 1.2325-16 & 0.0005+00\end{array}$

$0.0002+00$

$0.0005+00 \quad 7.8825-03$

$\begin{array}{lllll}2.00 & 0.0008+00 & 0.0005+00 & 0.000 \Sigma+00 & 0.000 \Sigma+00\end{array}$

$2.00 \quad 0.000 x+00 \quad 0.000 \Sigma+00 \quad 0.000 x+00$

$2.00 \quad 0.000 \Sigma+00 \quad 0.0005+00 \quad 0.000 \mathrm{x}+00$

$0.0002+00$
$0.000 E+00$

$0.0002+00$

$0.0008+00$

$\begin{array}{ll}0.000 \Sigma+00 & 0.000 \Sigma+00 \\ 0.000 z+00 & 0.000 z+00\end{array}$

$0.000 E+00 \quad 0.0005+00 \quad 0.0005+00 \quad 0.0005+00$

$0.0005+00 \quad 0.0005+00 \quad 0.0005+00$

$\begin{array}{llll}2.00 & 7.082 E-03 & 0.000 E+00 & 0.000 x+00\end{array}$

$0.0008+00$

$0.0005+00$

$0.000 \mathrm{E}+00$
$0.0005+00$

$\begin{array}{lll}2.00 & 0.000 E+00 & 0.0008+00 \\ 4.00 & 1.4938-02 & 0.0008+00\end{array}$

$0.0008+00 \quad 0.000 \mathrm{E}+00$

$0.0005+00$

$0.000 x+00$

$0.0002+00$

$1.693 \mathrm{E}-02 \quad 1.101 \mathrm{E}-16$

$0.000 x+00$

$0.000 \Sigma+00$
$0.000 \Sigma+00$

$0.0005+00 \quad 0.0005+00$

$0.000 E+00 \quad 0.000 x+00 \quad 0.0005+00$

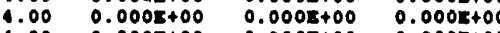

$1.00 \quad 0.0005+00 \quad 0.0005+00$

$0.0005+00 \quad 0.0005+00$

$0.000 E+00$ C.000E+00

$.000 x+00$

$0.0002+00$

$0.0002+00$

$0.0008+00$ 1.493E-02 1.101E-16

$0.000 \pi+00$

$0.000 \mathrm{x}+00$

$0.0005+00$

$0.0002+00$

$0.000 x+00$

$0.000 \Sigma+00 \quad 0.000 \pi+00 \quad 0.000 \Omega+00$

$0.000 z+00 \quad 0.0005+00 \quad 0.000 E+00$

$0.0002+00$

$\begin{array}{lll}0.000 \Sigma+00 & 0.000 E+00 & 0.000 z+00 \\ 0.000 \Sigma+00 & 0.000 \Sigma+00 & 0.000 \Sigma+00\end{array}$

$4.00 \quad 0.0002+00 \quad 0.000 \Sigma+00 \quad 0.000 \Sigma+00$

$\begin{array}{llll}.00 & 0.000 z+00 & 0.000 z+00 & 0.0008+00\end{array}$

$0.000 x+00$

$0.0008+00$

$1.4935-02$

$1.1018-16$

$0.0002+00$

$\begin{array}{lll}1.00 & 0.000 z+00 & 0.000 z+00\end{array}$

$0.0008+00$

$0.002=02$

$0.0002+00$

$0.0008+00$

$0.000 \mathrm{x}+00$

$6.00 \quad 0.0008+00 \quad 0.0008+00 \quad 0.000200$

$2.1225-02$
$0.000 E+00$

$0.0008+00$

$0.0005+00$

$0.000 \Sigma+00$

$0.000 \mathrm{E}+00$

$\begin{array}{llll}0.000 E+00 & 0.000 \Sigma+00 & 0.000 \Sigma+00 & 0.000 \Sigma+00 \\ 0.000 \Sigma+00 & 0.000 E+00 & 0.000 z+00 & 0.000 \Sigma+00\end{array}$

$0.000=00$

$0.000 z+00$

$0.000 z+00$

$0.0002+00$
$0.0000+00$

$0.0002+00$

$0.0005+00$
$2.1225-02$

$0.000 \mathrm{z}+00$

$0.000 \mathrm{E}+00$

$0.000 \mathrm{E}+00$

$0.000 E+00$

$0.0005+00$

$0.000 z+00 \quad 0.000 x+00$

$0.000 z+00 \quad 0.000 \Sigma+00$

$0.000 z+00$

$0.0005+00$
$0.000 E+00$

$0.000 E+00$
$0.000 E+00$

$6.00 \quad 0.000 E+00 \quad 0.000 \Sigma+00$

$0.000 E+00$

$0.000 E+00$

$0.000 E+00$
$0.000 E+00$

$0.000 E+00$
$0.000 E+00$

$0.000 E+00$
$0.000 z+00$

$\begin{array}{llll}6.00 & 0.000 \Sigma+00 & 0.000 z+00 & 0.000 \varepsilon+00 \\ 6.00 & 0.000 z+00 & 0.000 z+00 & 0.000 z+00\end{array}$

$0.000 E+00 \quad 0.0008+00 \quad 0.000 E+00$

$0.000 E+00$
$0.000 E+00$

$\begin{array}{llll}6.00 & 2.122 z-02 & 0.000 z+00 & 0.0008+00 \\ 6.00 & 0.000 z+00 & 0.000 z+00 & 0.000 z+00\end{array}$

$0.000 \mathrm{E}+00$

$0.000 E+00$

$0.000 E+00$

$0.0008+00$

$0.000 \Sigma+00$

$1.000=+00$

.

$1.000=+00$

$1.0005+00$

$1.2328-16 \quad 1.0008+00$

$0.000 x+00 \quad 1.000 x+00$

$1.1018-16 \quad 1.0008+00$

$0.0008+00 \quad 1.0008+00$

$0.000 z+00 \quad 1.000 x+00$

$1.000 z+00$

$1.000 x+00$

$1.1012-16 \quad 1.000 E+00$

$0.0002+00 \quad 1.000 E+00$

$1.000 \mathrm{z}+00$

$1.000 E+00$

$1.0002+00$

$0.000=+00 \quad 1.0002+00$

$0.840=17 \quad 1.0005+00$

$0.000=+00 \quad 1.000=+00$

$0.0008+00 \quad 1.0008+00$

$0.0008+00 \quad 1.0008+00$

$0.000 z+00 \quad 1.0005+00$

$9.8405-17 \quad 1.0005+00$

$0.000 z+00 \quad 1.000 z+00$

$1.000 z+00$

$0.0005+00 \quad 1.0005+00$

$0.000 \mathrm{E}+00 \quad 1.000 \mathrm{E}+00$

$.8408-17 \quad 1.0008+00$ 


\section{INSTRUCTIONS FOR USE OF GRAFXT}

GRAFXT was created to provide the DUST user with a means of rapidly analyzing the primary output, (concentrations and fluxes), of the code. GRAFXT takes the files TRACECND.DAT, TRACEFXD.DAT, and CONCNT.DAT and displays them on a video display device. The created graph is a linear $X-Y$ plot of the data.

GRAFXT was written using LAHEY FORTRAN version 5 and should work with any monitor. Although GRAFXT works on monochrome as well as color monitors, a color monitor is recommended as the display is greatly enhanced in this case.

GRAFXT is menu driven. Decisions on the variables to plot are resolved through interactive questioning of the user. As done in the DUSTIN chapter, text that appears on the console will appear in a double border box. Responses by the user will be italicized within this box.

\subsection{Selection of the File for Plotting}

The first series of questions involves determining which file should be used for plotting purposes.

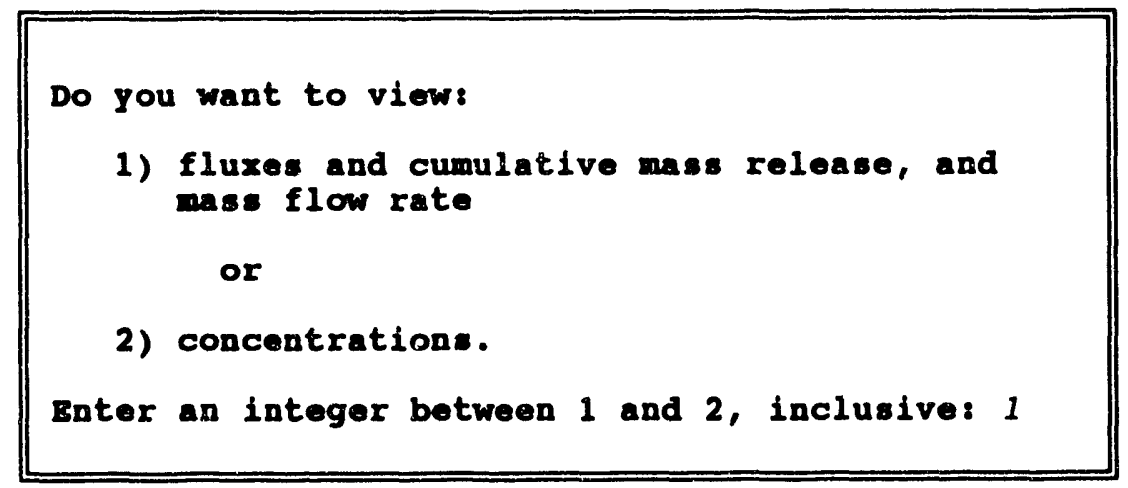

In this case, TRACEFXD.DAT was selected. If the user selected concentrations, the code would ask the question which conceritration file (TRACECND.DAT or CONCNT.DAT) should be opened.

\subsection{Selection of the Plot Variables}

After selecting the file, the type of plot must be determined. GRAFXT can take any one of the three files and plot the variables versus time or location. In general, the trace files, which have the variables at fixed locations at a large number of time steps, were designed to be plotted versus time at fixed locations. The CONCNT.DAT file was written to provide a plot of concentration versus location at fixed times. 


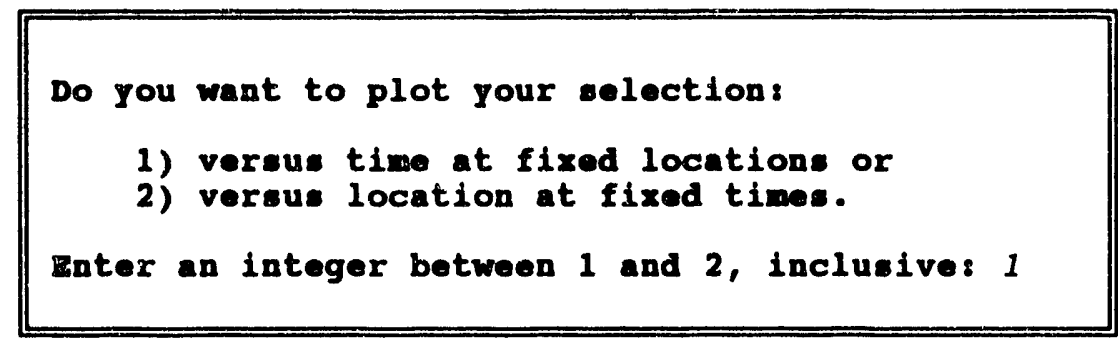

\subsubsection{Number of plots per graph - Independent variable is time}

In this case, the user selected a plot with time as the independent variable. At this point, the data file is read, the title card which labels each plot is printed and the number of trace nodes on the file are printed. The user is asked to provide the nodes at which a plot is requested. More than one plot can be drawn on the graph at one time. For color monitors, it is recommended that the number of plots does not exceed the number of different colors available. For monochrome monitors, it is recommended that only a few plots per graph be made due to difficulty in differentiating between plots.

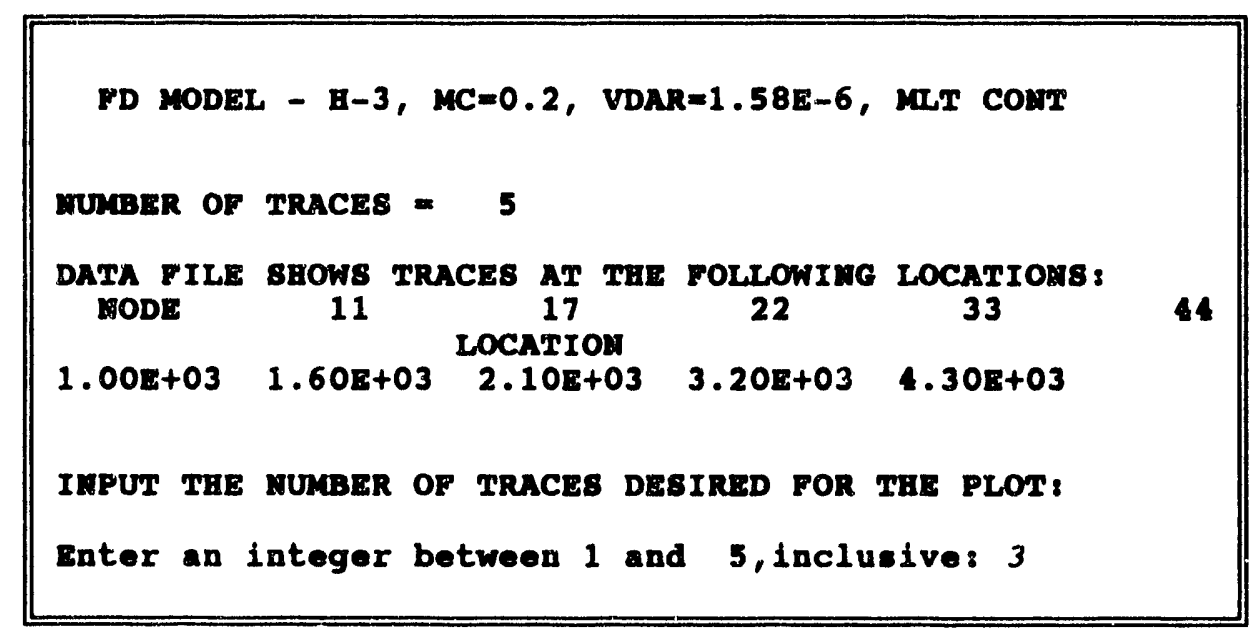

In the previous example, 3 plots are requested. The code then prompts the user for the node numbers to be used for plotting.

INPUT THE NODE NUMBERS FOR THE TRACES:

172233 
The node numbers can be input on a single line, separated by a space as in the example. If the code user requested that all five nodes be plotted, the code would automatically define the five plot nodes and the previous question is skipped.

\subsubsection{Number of plots per graph - Independent variable is distance}

If the user requested distance as the independent variable, the GRAFXT code asks a different series of questions. Namely, it determines the fixed times at which a plot is requested. The code first prints the locations of all of the nodes in the file. Then the table of output times is printed.

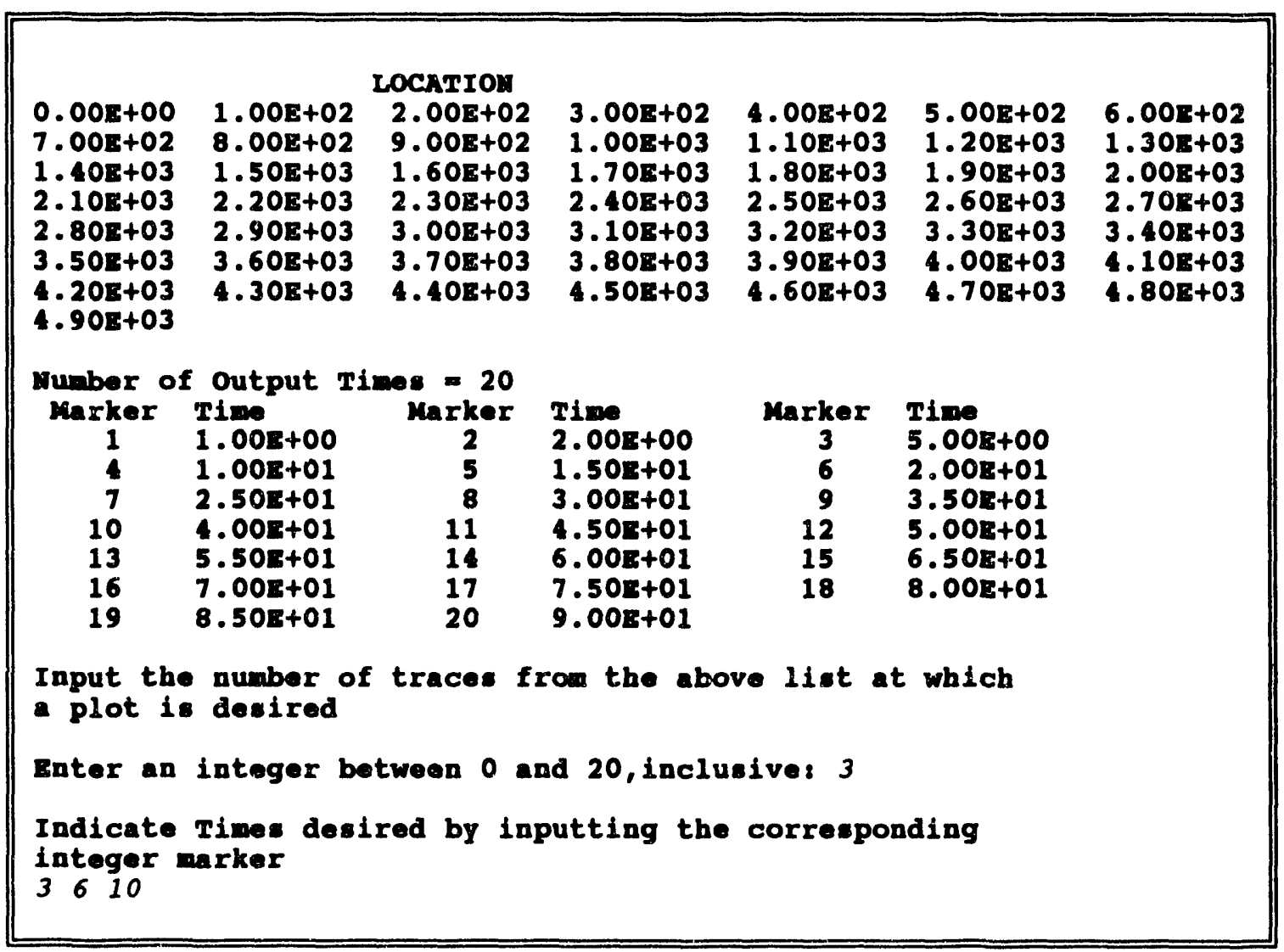

In this example, the file contained 20 output times. The times at which a plot are requested are determined through the integer markers. This was done to prevent problems with comparisons between real numbers as the code checks to make sure that the requested time is in the output file. Therefore, specifying 3,6 , and 10 as the plot markers gives output at times of 5,20 and 40 years. 


\subsection{Creating a Plot}

At this point, the code prompts the user if the data should be plotted as is, (automatic generation) or if the user wants to customize the plot.

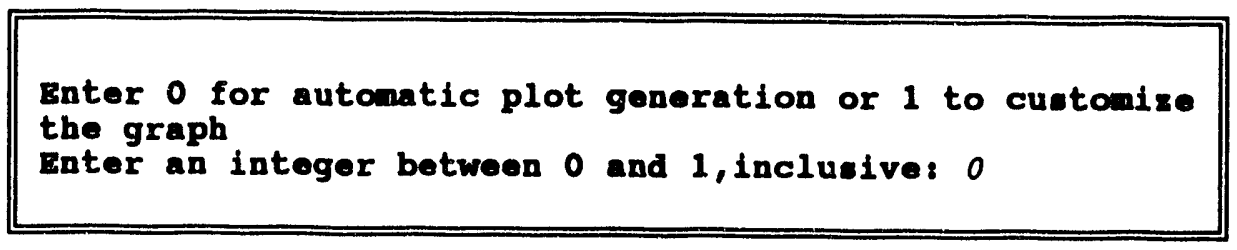

If automatic generation is used, the screen is changed to graphics mode and the plot is drawn.

Figure 9.1 displays a reproduction of a typical plot of flux versus time generated by GRAFXT. GRAFXT plots are on the video display and not easily reproduced on a hard copy. Therefore, a reproduction of the plot was created using the spreadsheet QUATTRO.PRO. In Fig. 9.1, the Y-Axis label, FLUX X $10^{\wedge}-15$, is at the top. This is done to prevent the need to write parallel to the axis using FORTRAN, which is quite cumbersome. The value for the exponent, -15 , in this label is calculated by the code and is problem dependent. Beneath the $\mathrm{Y}$-axis label, is the problem title supplied from the input file. The $\mathrm{X}$-axis is written beneath the values marked on this axis. The generation and labeling of all axis is performed automatically by the code and depends on the maximum and minimum values present. If the flux has both positive and negative values at different locations, this is handled automatically by the code. Although, different traces are highlighted with different markers in this figure. The actual output of GRAFXT does not include markers for data points. Curves are differentiated using different colors on the plot. Another difference between Figure 9.1 and a GRAFXT plot is the labels for the nodes appears on the left when GRAFXT is used.

After plotting, hitting any key will clear the screen and return to text mode.

\subsection{Customizing a Plot}

A plot can be customized in three ways, a) moving the location of the plot on the screen, b) redefining the axis labels, and c) limiting the range of the data. If customization is requested the following questions appear. 
FD Model $-\mathrm{H}-3, \mathrm{MC}=0.2, \mathrm{VDAR}=1.58 \mathrm{E}-6$ FLUXX $10^{\wedge}-15$

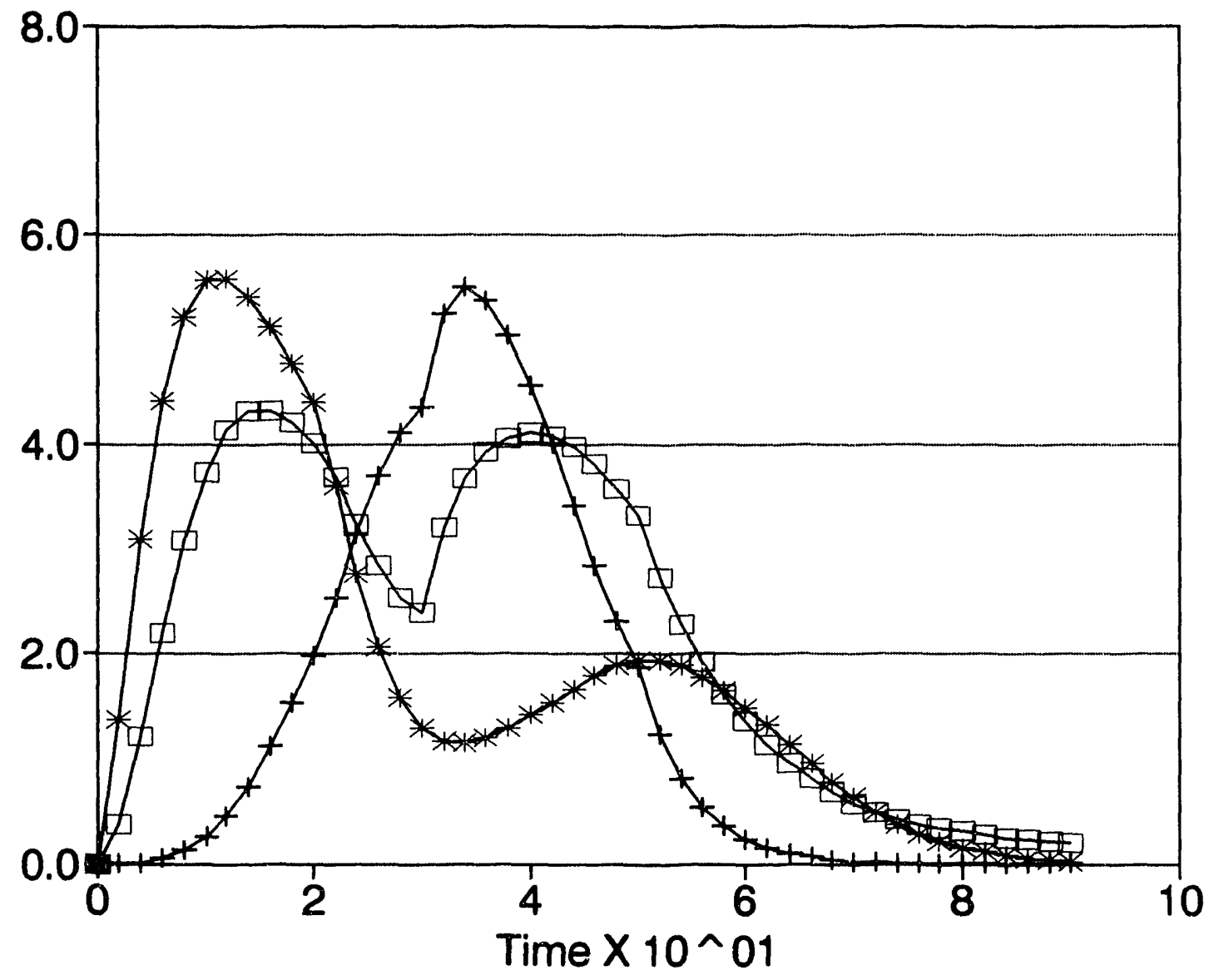

Figure 9.1 Typical graph created by the program GRAFXT. 


\subsubsection{Changing the Plot Origin}

The code GRAFXT selects the screen origin of the plot automatically. The value is chosen to get the maximum resolution on the screen. Under unusual circumstances, this may cause the axis labels to be off the screen. In this case, the origin can be redefined. The code asks the following question:

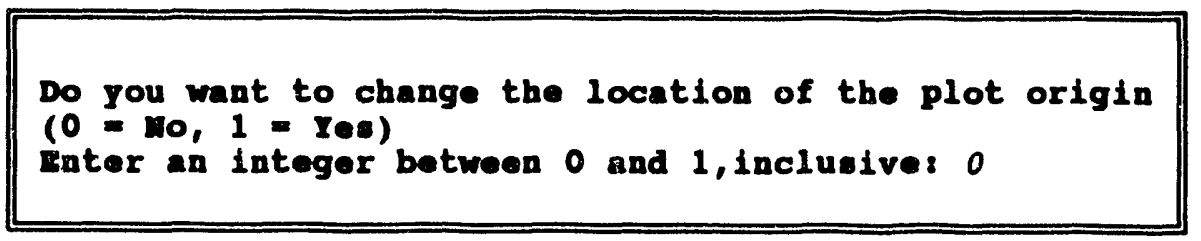

If the answer is yes, the code asks for the number of inches to move the $X$ and $Y$ coordinates of the origin.

\subsubsection{Changing the Axis Labels}

The code provides default labels for both the $\mathrm{X}$ and $\mathrm{Y}$ axis. If these are inadequate, they can be changed by responding 1 to the following question:

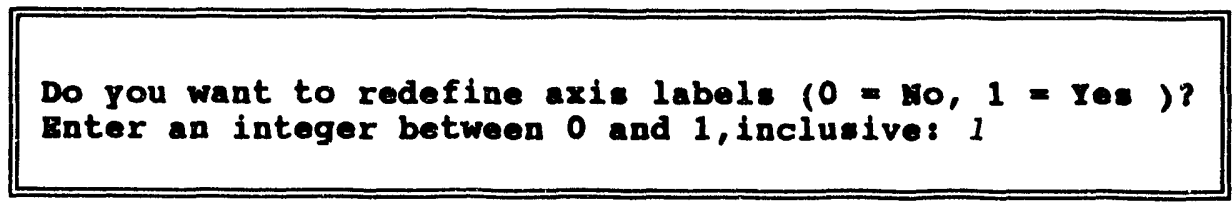

In this case, the code prompts the user for the both the $\mathrm{X}$ and $\mathrm{Y}$ axis labels. These are restricted to 20 characters in length.

\subsubsection{Limiting the range of the data}

GRAFXT provides a linear X-Y plot of the data. When automatic generation is used, all of the data is plotted on the graph. This causes poor resolution away from peak values. GRAFXT provides the user with a means of increasing the resolution at any point in the graph through specifying maximum and minimum values for both the $X$ and $Y$ parameters. The procedure follows: 


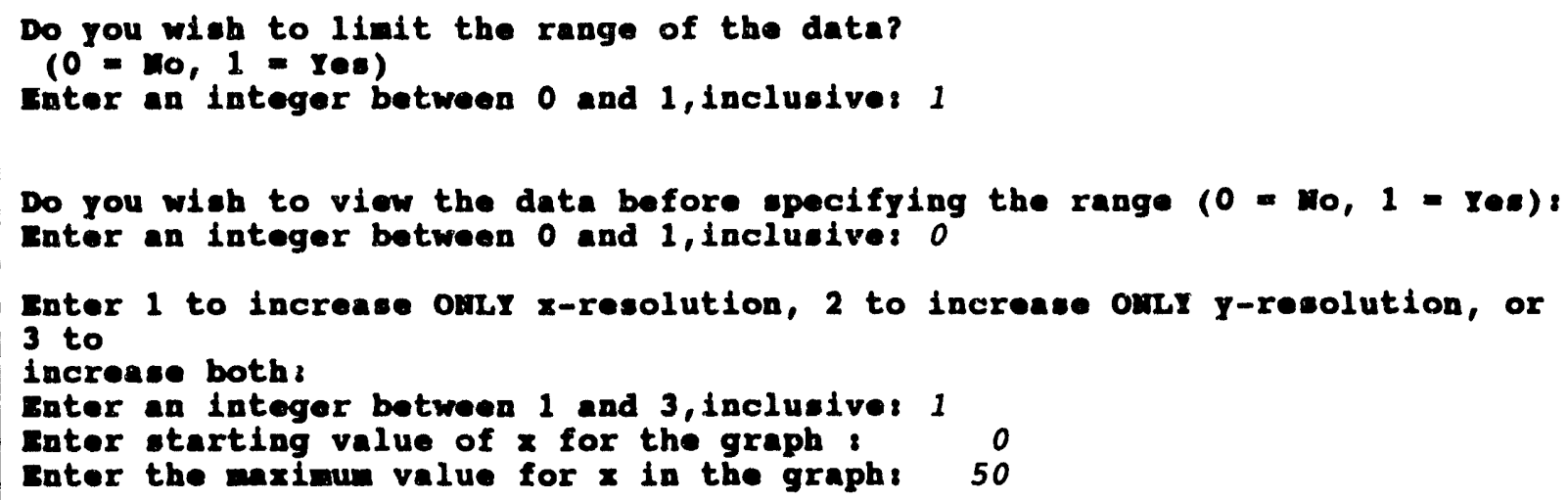

After a decision has been made to limit the data, the user is given the opportunity to view the selected plot data. If a value of 1 is input, all of the data at each of the plot locations is printed. This is useful when trying to determine appropriate ranges for the data. After this section, the user is provided three options for limiting the range of the data as presented above. In the example, the user excludes all points in which the value of $X$ is outside the range of 0 50. At this point, the user is asked to view the data before plotting. If this option is selected, only data that falls within the specified range is printed. If the range is inappropriate, the user can return and specify a new range. After the user is satisfied with the data, a plot is generated.

Use of this option is particularly useful in two cases: a) often in a simulation, releases are peaked around a small change in time. If this happens, the minimum and maximum values for time can be changed to provide better resolution about the peak. And, b) In certain cases, the values away from the peak are required. Due to the linear plot, these appear as near zero. By limiting the range of times to be far away from the peak, higher resolution can be obtained in these areas.

\subsection{Creating Additional Plots}

Although GRAFXT can creates one plot at any given time, after a plot has been viewed, the following question appears:

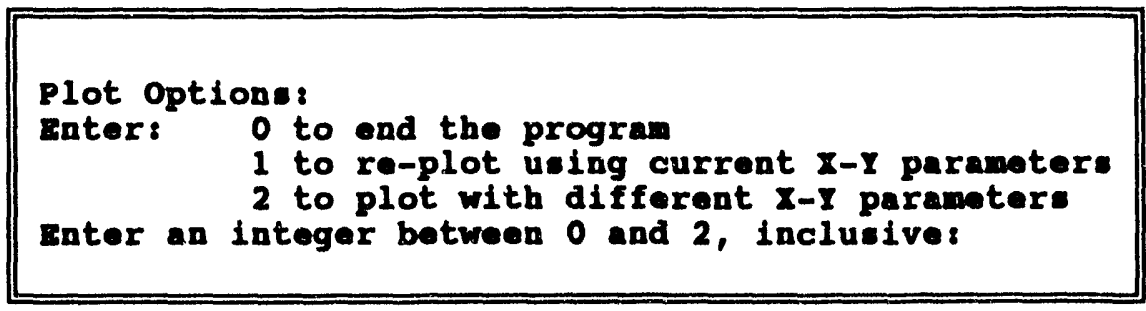


Option 0 ends the program. Option 1 permits further plots to be made with the current plot file. For example, if the previous plot was the flux versus time at specified locations, the user could request another plot with a restricted range of data, or create a similar plot with different trace locations. For the flux file, TRACEFXD, the user could also use the same locations and plot the cumulative mass release or the mass flow rate. With this option, the user can not change from plots of the function versus time to plots versus location, or vice versa. Option 2 returns the user to the initial menu and is equivalent to exiting the code and beginning over. All plot options are available. 


\section{CONCLUSIONS}

The DUST code, which predicts the release and transport of radioactive contaminants from a LLW disposal facility, has been developed, implemented, and tested. The DUST code contains models to predict the important processes (fluid flow, container degradation, wasteform leaching, and radionuclide transport) related to release and transport of radionuclides within the disposal facility.

The DUST code improves upon existing codes in that more flexibility is allowed in order to model the various waste stream/wasteform/container systems while still retaining relatively simple models that do not require extensive computer time or provide an undue burden on the code user in terms of input requirements.

To facilitate creation oi an input deck, the code DUSTIN has been written. DUSTIN is a menu driven program that guicies the user through all of the steps required to create an input deck. Upon completion of these steps, DUSTIN will write an input file for use by DUST.

In addition, to permit rapid analysis of the output data created by DUST, a graphics program GRAFXT has been written. GRAFXT is capable of plotting the concentration, flux, or mass that has passed a fixed location as a function of either time or location directly on a video console.

The DUST code improves upon the codes that currently exist, yet still retains a simplified structure in their treatment of the complex phenomena involved in the release and transport of radionuclides within a disposal facility. As such, the models are prone to misuse through improper choice of the input parameters. Enough emphasis cannot be placed on the need for justification and documentation of the choices for the input parameters. The model predictions are only as good as the least justifiable input.

In using DUST, as with all computer models, the validity of the predictions relies heavily on the validity of the input parameters. Often, the largest uncertainties arise from uncertainty in the input parameters. Therefore, it is crucial to document and support the use of these parameters. The DUST code, because of its ability to compute release rates quickly, will be extremely useful for screening to determine the radionuclides released at the highest rate, parameter sensitivity analyses, and, with proper choice of the input parameters, provide upper bounds to release rates. 


\section{REFERENCES}

[ANS, 1986]

American Nuclear Society, "Measurement of the Leachability of Solidified Low-Level Radioactive Wastes," Prepared by the American Nuclear Society Standards Committee Working Group ANS-16.1, 1986.

[Baes, 1983]

C.F. Baes, III, and R.D. Sharp, "A Proposal for Estimation of Soil Leaching and Leaching Constants for Use in Assessment Models," Journal of Environmental Quality, Vol. 12, No. 1, pp. 17-24, 1983.

[Chu, 1991]

M.S.Y. Chu, M.W. Kozak, J.E. Campbell, and B.M. Thompson, "A Self-Teaching Curriculum for the NRC/SNL Low-Level Waste Performance Assessment Methodology," NUREG/CR5539, SAND90-0585, Sandia National Laboratories, 1991.

[Clifton, 1989]

J.R. Clifton and L.I. Knab, "Service Life of Concrete," NUREG/CR-5466, NISTIR89-4086, National Institute of Standards and Technology, 1989.

[Cowgill, 1992]

M.G. Cowgill and T.M. Sullivan, "Source Term Evaluation for Low-Level Radioactive Waste Disposal Performance," NUREG/CR-5911, BNL-NUREG-52334, Brookhaven National Laboratory, 1993.

[Cowgill, 1992a]

M.G. Cowgill and T.M. Sullivan, "Source Term Evaluation for Performance Assessment of LLW Disposal," WM '92, ed. R.G. Post, Tucson, AZ, 1992.

[DOE, 1987]

Conference of Radiation Control Program Directors, Inc., "Compilation of State-by-State LowLevel Radioactive Waste Information," DOE/ID/12377-T1, October, 1987.

[DOE, 1990]

U.S. Department of Energy, "Integrated Data Base for 1990: U.S. Spent Fuel and Radioactive Waste Inventories, Projections, and Characteristics," DOE/RW-0006, Rev. 6, Oak Ridge National Laboratory, October, 1990.

[FR, 1982]

Federal Register, "Licensing Requirements for Land Disposal of Radioactive Waste," Vol. 47, No.248, Monday, December 27, 1982. 
[Gee, 1988]

G.W. Gee and D. Hillel, "Groundwater Recharge in Arid Regions: Review and Critique of Estimation Methods," Hydrological Processes, Vol. 2, p. 255-266, 1988.

[Gerhold, 1981]

W.F. Gerhold, E. Escalante, and B.T. Sanderson, "The Corrosion Behavior of Selected Stainless Steels in Soil Environments," NBSIR 81-2228, National Bureau of Standards, 1981.

[Gruhlke, 1986]

J.M. Gruhlke, J. Neiheisel, and L. Battist, "Estimates of the Quantities, Form and Transport of Carbon-14 in Low-Level Radioactive Waste," EPA 520/1-86-019, U.S. Environmental Protection Agency, 1986.

[Higginbotham, 1983]

L.R. Higginbotham, "Final Waste Classification and Waste Form Technical Position Papers," United States Nuclear Regulatory Commission, May 11, 1983.

[Huyakorn, 1989]

P.S. Huyakorn, J.B. Kool, and J.B. Robertson, "VAM2D - Variably Saturated Analysis Model in Two Dimensions," NUREG/CR-5352, HGL/89-01, HydroGeoLogic, Inc., 1989.

[Kozak, 1989]

M.W. Kozak, et. al., "Background Information for the Development of a Low-Level Waste Performance Assessment Methodology: Selection and Integration of Models," NUREG/CR-5453, SAND89-2509, Volume 3, Sandia National Laboratories, 1989.

[Kozak, 1989a]

M.W. Kozak, et. al., "Background Information for the Development of a Low-Level Waste Performance Assessment Methodology: Identification and Recommendation of Computer Codes," NUREG/CR-5453, SAND89-2509, Volume 4, Sandia National Laboratories, 1989.

[Kozak, 1990]

M.W. Kozak, et. al., Background Information for the Development of a Low-Level Waste Performance Assessment Methodology: Computer Code Implementation and Assessment," NUREG/CR-5453, SAND89-2509, Volume 5, Sandia National Laboratories, 1990.

[Kunz, 1982]

C.O. Kunz, "Radioactive Gas Production and Venting at a Low-Level Radioactive Waste Burial Site," Nucl. Chem. Waste Management, Vol. 3, p. 185, 1982.

[Lohaus, 1991]

P.H. Lohaus, "Technical Position on Waste Form, Revision 1," U.S. Nuclear Regulatory Commission, January 24, 1991. 
[Looney, 1987]

B.B. Looney, M.W. Grant, and C.M. King, "Estimation of Geochemical Parameters for Assessing Subsurface Transport at the Savannah River Plant," DPST-85-904, DE87-013051, E.I. duPont de Nemours \& Co., 1987.

[Matuszek, 1983]

J.H. Matuszek and L.W. Robinson, "Respiration of Gases from Near-Surface Radioactive Waste Burial Trenches," Waste Management '83, ed. R.G. Post, pp. 423 - 427, Tucson, AZ, 1983.

[Mughabghab, 1989]

S.F. Mughabghab and T.M. Sullivan, "Evaluation of the Pitting Corrosion of Carbon Steels and Other Ferrous Metals in Soil Systems," Waste Management, Vol. 9, pp. 239-251, 1989.

[Otzunali, 1981]

O.I. Otzunali, G.C. Re', P.M. Moskowitz, E.D. Picazo, and C.J. Pitt, "Data Base for Radioactive Waste Management: Impact Analyses Methodology Report," NUREG/CR-1759, Vol. 3, Dames and Moore, 1981.

[Otzunali, 1986]

O.I. Oztunali and G.W. Roles, "Update of Part 61 Impacts Analysis Methodology," NUREG/CR4370, Vol. $1,1986$.

[Roache, 1976]

P.J. Roache, Computational Fluid Dynamics, Hermosa Publishers, Albuquerque, NM, 1976.

[Roles, 1990]

G.W. Roles, "Characteristics of Low-Level Radioactive Waste Disposed During 1987 Through 1989", NUREG-1418, U.S. Nuclear Regulatory Commission, 1990.

[Romanoff, 1957]

M. Romanoff, "Underground Corrosion," National Bureau of Standards Circular 579, 1957.

[Shuman, 1991]

R. Shuman, N. Chau, and V. Rogers, "Improved Modeling of Engineered Barriers for Low-Level Waste Disposal," preserited at Waste Management '91, Tucson, AZ, 1991.

[Soo, 1990]

P. Soo and L.W. Milian, "The Impact of LWR Decontaminations on Solidification, Waste Disposal and Associated Occupational Exposure," NUREG/CR-3444, BNL-NUREG-51699, Vol. 7, Brookhaven National Laboratory, 1990. 
[Starmer, 1988]

R.J. Starmer, L.R. Deering, and M.F. Weber, "Performance Assessment Strategy for Low-Level Waste Disposal Sites," in the proceedings of the Tenth Annual DOE Low-Level Waste Management Conference, Denver, 1988.

[Streigel, 1985]

R.G. Streigel, "Methods for Determining the Transport of Radioactive Gases in the Unsaturated Zone," Department of Energy Low-Level Waste Management Participants Meeting, p. 579 - 587, Denver, CO., 1985.

[Sullivan, 1988]

T.M. Sullivan, C.R. Kempf, C.J. Suen, and S.M. Mughabghab, "Low-Level Radioactive Waste Source Term Model Development and Testing," NUREG/CR-5204, BNL-NUREG-52160, Brookhaven National Laboratory, 1988.

[Sullivan, 1988a]

T.M. Sullivan, C.J. Suen, and E. Sweeney, "Low-Le el Waste Source Term Evaluation Quarterly Progress Report: July - September, 1988," WM-3276-7, Brookhaven i iational Laboratory, 1988.

[Sullivan, 1989]

T.M. Sullivan and C.J. Suen, "Low-Level Waste Shallow Land Disposal Source Term Model: Data Input Guides," NUREG/CR-5387, 3NL-NUREG - 32206, Brookhaven National Laboratory, 1989.

[Sullivan, 1991]

T.M. Sullivan and C.J. Suen, "Low-Level Waste Source Term Model Development and Testing," NUREG/CR-5681, BNL-NUREG-52280, Brookhaven National Laboratory, 1991.

[Sullivan, 1991a]

T.M. Sullivan, "Selection of Models to Calculate the LLW Source Term," NUREG/CR-5773, BNL-NUREG-52295, Brookhaven National Laboratory, 1991.

[Sullivan, 1991b]

T.M. Sullivan and M.G. Cowgill, "Evaluation of the Data Available for Estimating Release Rates from Commercial Low-Level Waste Packages," BNL-NUREú-4676, DOE Low-Level Waste Management Conference, November 1991, Atlanta, GA.

[Sullivan, 1992]

T.M. Sullivan, "Development of DUST: A Computer Code that Calculates Release Rates from a LLW Disposal Unit," Waste Management '92, ed. R.G. Post, Tucson, AZ, 1992. 
[Thibault, 1990]

D.H. Thibault, M.I. Sheppard, and P.A. Smith, "A Critical Compilation and Review of Default Soil/Solid Liquid Partition Coefficients, $K_{d}$, for Use in Environmental Assessments," AECL10125, Atomic Energy of Canada Limited, 1990.

[Travis, 1991]

B.T. Travis and K.H. Birdsell, "TRACR3D: A Model of Flow and Transport in Porous Media: Model Description and User's Manual," LA-11798-M, Los Alamos National Laboratory, 1991.

[Van Genuchten, 1978]

M. Th. Van Genuchten, "Mass Transport in Saturated-Unsaturated Media: One-Dimensional Solution," Research Report 78-WR-11, Water Resources Program, Department of Civil Engineering, Princeton University, 1978.

[Walton, 1990]

J.C. Walton, L.E. Plansky, and R.W. Smith, "Models for Estimation of Service Life of Concrete Barriers in Low-Level Radioactive Waste Disposal," NUREG/CR-5542, EGG-2597, EG\&G Idaho National Engineering Laboratory, 1990.

[Yeh, 1987]

G.T. Yeh, "FEMWATER: A Finite Element Model of Water Flow Through SaturatedUnsaturated Porous Media - First Revision," ORNL-5567/R1, Oak Ridge National Laboratory, 1987. 


\section{APPENDIX A \\ MATHEMATICAL DESCRIPTION OF THE MODELS SELECTED FOR SOURCE TERM ANALYSIS}

The mathematical details pertaining to the models selected for source term analysis are described in this Appendix. The models that have involved mathematical descriptions cover the processes of wasteform diffusion release, and radionuclide transport. The complete models for water flow, container degradation, rinse release, and uniform release are presented in Chapter 2.

There are no models, per se, for the disposal facility radionuclide inventory. Rather, the inventory will be treated as a known quantity. The methodology required to obtain the inventory is also discussed in Chapters 2 and 3.

\section{Wasteform Release}

\section{Diffusion-Controlled Release}

Conceptually, release from many solidified wasteforms can be described as a diffusioncontrolled process. Two models, differing only in geometry, are provided. In these models, it is assumed that, at the outer edge of the wasteform, the radionuclide concentration is zero. This assumes that transport processes away from the wasteform are fast enough to remove any radionuclides supplied by diffusion out of the wasteform. Although this situation will never be realized exactly, it does provide for the maximum diffusive release rate from the wasteform. Further, in many cases of practical interest, the solution concentration outside of the wasteform will not increase to a level such that it will markedly influence release.

In both models, we analytically solve the diffusion equation corrected for decay.

$$
\frac{\partial C}{\partial t}=\nabla \cdot D \nabla C-\lambda C
$$

where $D=$ the effective diffusion coefficient, $C$ is the concentration, and $\lambda$ is the radioactive decay constant. 
The initial condition assumes a uniform concentration throughout the wasteform:

$$
C(x, y, z, 0)=C_{0}
$$

The boundary conditions assume symmetry about the midplane of the wasteform and zero concentration at the outer edge.

$$
\begin{aligned}
& C\left(x_{b}, y, z, t\right)=0 \\
& C\left(x, y_{b}, z, t\right)=0 \\
& C\left(x, y, z_{b}, t\right)=0
\end{aligned}
$$

where the subscript $b$ denotes a boundary.

Solution of Eqn. (A.1) subject to the initial and boundary conditions gives the concentration at any location within the wasteform. However, the quantity of interest is the release rate, which is the mass flux integrated over the surface area.

$$
Q(t)=\int d S \cdot J_{s}
$$

where:

$Q(t)$ is the mass release per unit time; and

$\mathrm{J}_{\mathrm{s}}$ is the mass flux at the surface.

For one-dimensional diffusion-controlled release,

$$
J_{s}=-D \frac{\partial C\left(x_{s}\right)}{\partial x}
$$

where $\mathbf{x}_{\mathrm{s}}$ denotes a surface of the wasteform. 


\section{Cylindrical Geometry}

The expression for the mass release rate from a cylindrical wasteform of height, $2 \mathrm{~L}$, and radius $R$ is [Pescatore, 1991]:

$$
Q(t)=Q_{r}(t)+Q_{z}(t)
$$

where:

$$
Q_{r}(t)=Q_{r}(\lambda ; t)=\frac{32}{\pi^{2} R^{2}} \cdot D C_{0} V e^{-\lambda t} S_{p}(t) S_{q}(t)
$$

and

$$
Q_{z}(t)=Q_{z}(\lambda ; t)=\frac{8}{L^{2}} \cdot D C_{o} V e^{-\lambda t} S_{c}(t) S_{d}(t)
$$

Here,

$$
\begin{aligned}
& S_{c}(t)=\sum_{m=1}^{\infty} \frac{e^{-(\beta m / R)^{2} D t}}{\left(\beta_{m}\right)^{2}} \\
& S_{p}(t)=\sum_{n=1}^{\infty} \frac{e^{-[(2 n-1) \pi t 2 L]^{2} D t}}{(2 n-1)^{2}} \\
& S_{q}(t)=\sum_{m=1}^{\infty} e^{-(\beta m / R)^{2} D t} \\
& S_{d}(t)=\sum_{n=1}^{\infty} e^{-[(2 n-1) \pi / 2 L]^{2} D t}
\end{aligned}
$$


where the $\beta_{m}$ are the zeroes of the zero-th order cylindrical Bessel function and are presented in Table A.1.

Table A.1 Values of the parameters $\beta_{\mathrm{m}}$ for $m=1$ to 20 .

These parameters satisfy the equation $\mathrm{J}_{0}\left(\beta_{\mathrm{m}}\right)=0$, with $\mathrm{J}_{0}(\mathbf{x})$ the zeroth order cylindrical Bessel function.

\begin{tabular}{cccc}
\hline $\mathrm{m}$ & $\beta_{\mathrm{m}}$ & $\mathrm{m}$ & $\beta_{\mathrm{m}}$ \\
\hline & & & \\
\hline 1 & 2.4048255577 & 11 & 33.7758202136 \\
2 & 5.5200781103 & 12 & 36.9170983537 \\
3 & 8.6537279129 & 13 & 40.0584257646 \\
4 & 11.7915344391 & 14 & 43.1997917132 \\
5 & 14.9309177086 & 15 & 46.3411883717 \\
6 & 18.0710639679 & 16 & 49.4826098974 \\
7 & 21.2116366299 & 17 & 52.6240518411 \\
8 & 24.3524715308 & 18 & 55.7655107550 \\
9 & 27.4934791320 & 19 & 58.9069839261 \\
10 & 30.6346064684 & 20 & 62.0484691902 \\
& & & \\
\hline
\end{tabular}

Efficient means of calculating the infinite series in Eqns. (A.9 - A.12) have been developed [Pescatore, 1991] and are implemented in the source term code.

\section{$\underline{\text { Rectangular Geometry }}$}

Given a rectangular-shaped, radioactive wasteform with dimensions $2 a, 2 b$, and $2 c$ along the $x, y$, and $z$ directions, respectively, and given the same assumptions about the wasteform as were utilized earlier for the cylindrical case, the concentration of radioactive species in the rectangular block is as follows:

$$
C(x, y, z, t)=\frac{64}{\pi^{3}} C_{o} e^{-\lambda s} T_{x}(a, t) T_{y}(b, t) T_{z}(c, t)
$$


where the generic function $T_{u}(L, t)$ represents the open series:

$$
T_{u}(L, t)=\sum_{n=1}^{\infty}(-1)^{n+1} \cdot \frac{e^{-((2 n-1) \pi / 2 L]^{2} D t}}{2 n-1} \cdot \cos \left(\frac{2 n-1}{2 L} \pi u\right)
$$

Equation (A.13) is obtained from the mathematically analogous expression for the temperature distribution within a heat-conducting parallelepiped.

Following [Pescatore, 1991], the release rate per unit area across the face $x=a$ of the wasteform is then:

$$
-\left.D \frac{\partial C}{\partial x}\right|_{x=a}=\frac{64}{\pi^{3}} C_{o} D e^{-\lambda} T_{y}(b, t) T_{z}(c, t)\left[\frac{\pi}{2 a} S_{d}(a, t)\right]
$$

where the function $S_{d}(a, t)$ is the open series [A.12] with $L=a$. The total release rate, $Q_{a}$, across the face $x=a$ is obtained upon integration of Eqn. [A.15] over the entire area of this face, yielding:

$$
Q_{x=a}(\lambda ; t)=\frac{512}{\pi^{4}} C_{o} D \frac{b c}{a} e^{-\lambda} S_{p}(b, t) S_{p}(c, t) S_{d}(a, t)
$$

where the functions $S_{p}(b, t)$ and $S_{p}(c, t)$ represent the open series (A.10) evaluated with $L=b$ and $\mathrm{L}=\mathrm{c}$, respectively. Analogous expressions for the total release rates from the other surfaces of the wasteforms can be obtained by substituting the dimensions of those surfaces in Eqn. (A.16).

\section{Radionuclide Transport}

Migration of contaminants through the disposal facility will be modeled in one of two ways: the multi-cell mixing cascade model or the finite difference solution of the advectiondispersion equation.

The mixing cell cascade approach is a generalization of the work performed by Sandia National Laboratories [Kozak, 1990] and relies on an analytical solution of the appropriate equations. With the analytical solution, the release rates from the disposal facility at any time are relatively easy to estimate. However, in order to obtain these solutions some restrictions on the generality of the problem must be made. 
Both models begin with a mass balance for the contaminant within a control volume:

$$
\begin{aligned}
& \frac{\partial}{\partial t}(\theta C)=-\frac{\partial J}{\partial x} \\
& -\lambda(\theta C+\rho S)-\frac{\partial}{\partial t}(\rho S) \\
& +q
\end{aligned}
$$

where:

$C=$ solution concentration;

$\theta=$ the volumetric moisture content of the region (dimensionless);

$\lambda=$ radioactive decay constant;

$\mathrm{S}=$ adsorbed concentration, the mass adsorbed per unit mass of the solid;

$\rho=$ bulk density of the solid; and

$\mathrm{q}=$ source/sink term used to model release from the waste form.

In Eqn. (A.17) we assume that the mass adsorbed on the solid surfaces is in equilibrium with the mass in solution. Further, we assume that this equilibrium can be described using a concentration independent partition coefficient, $\mathrm{K}_{d}$, as follows:

$$
S=K_{d} C
$$

Using the above relationship for $S$, assuming that the bulk density remains constant within the disposal facility and rearranging Eqn. (A.17), the following equation is obtained:

$$
\begin{aligned}
& \frac{\partial}{\partial t}(R \theta C)=-\frac{\partial J}{\partial x} \\
& -\lambda \theta R C+q
\end{aligned}
$$


where:

$$
R=1+\frac{\rho K_{d}}{\theta}
$$

$\mathrm{R}$ is known as the retardation coefficient.

The rate of change in mass flux with position provides a measure of the flow rate of mass entering or leaving the control volume and contains terms related to movement through the processes of diffusion/dispersion and advection. The mass flux is:

$$
J=-\theta D \frac{\partial C}{\partial x}+V_{d} C
$$

where:

D = the diffusion-dispersion coefficient,

$$
D=D_{m}+\frac{a_{t}\left|V_{d}\right|}{\theta} ;
$$

$\mathrm{D}_{\mathrm{m}} \quad$ = effective diffusion coefficient;

$\mathrm{a}_{\mathrm{t}} \quad=$ transverse dispersivity;

$\mathrm{V}_{\mathrm{d}} \quad=$ Darcy velocity;

Using Eqn. (A.21) in the mass balance equation with a linear sorption isotherm, Eqn. (A.19), yields:

$$
\begin{aligned}
\frac{\partial}{\partial t}(R \theta C) & =\frac{\partial}{\partial x}\left(\theta D \frac{\partial C}{\partial x}\right)-\frac{\partial}{\partial x}\left(V_{D} C\right) \\
& -\lambda \theta R C+q
\end{aligned}
$$




\section{Mixing-Cell Cascade}

The mixing cell cascade model divides the disposal facility into a number of uniform size mixing cells as depicted in Figure A.1. Within each cell it is assumed that the contaminant released from the waste form is uniformly mixed thereby giving a uniform solution concentration. In order to obtain an analytical solution the following assumptions are made:

a) Migration is dominated by advective flow and therefore diffusion and dispersion can be ignored.

b) The advection velocity, moisture content, and the retardation coefficient are constant throughout the disposal facility. Although these parameters will show variations due to different materials in the facility, they should be a selected to provide a representative average for the entire facility. becomes:

Using these assumptions in Eqn. (A.22) the transport equation for the $\mathrm{i}^{\text {th }}$ mixing cell

$$
\frac{\partial C_{i}}{\partial t}=-\frac{V_{D}}{\theta R} \frac{\partial C_{i}}{\partial x}-\lambda C_{i}+\frac{q_{i}}{\theta R}
$$

For a disposal facility of height $H$, there are $N$ mixing cells of height $h(h=H / N)$. Using this definition of the size of the mixing cell and upwind differencing (because migration is controlled by advection) to estimate the spatial derivative, Eqn. (A.23) becomes:

$$
\frac{d C_{i}}{d t}=-\alpha N\left(C_{i}-C_{i-1}\right)-\lambda C_{i}+\beta N Q_{i}
$$

where:

$\mathrm{Q}=$ the total release rate from the wasteform;

$\alpha=\mathrm{V}_{\mathrm{d}} /(\theta \mathrm{RH})$;

$\beta=1 /\left(\theta R_{H} A_{f}\right)$; and

$A_{f}=$ the area of the facility.

In this description, $\mathrm{HA}_{f} / \mathrm{N}$ is the volume of a single mixing cell. 

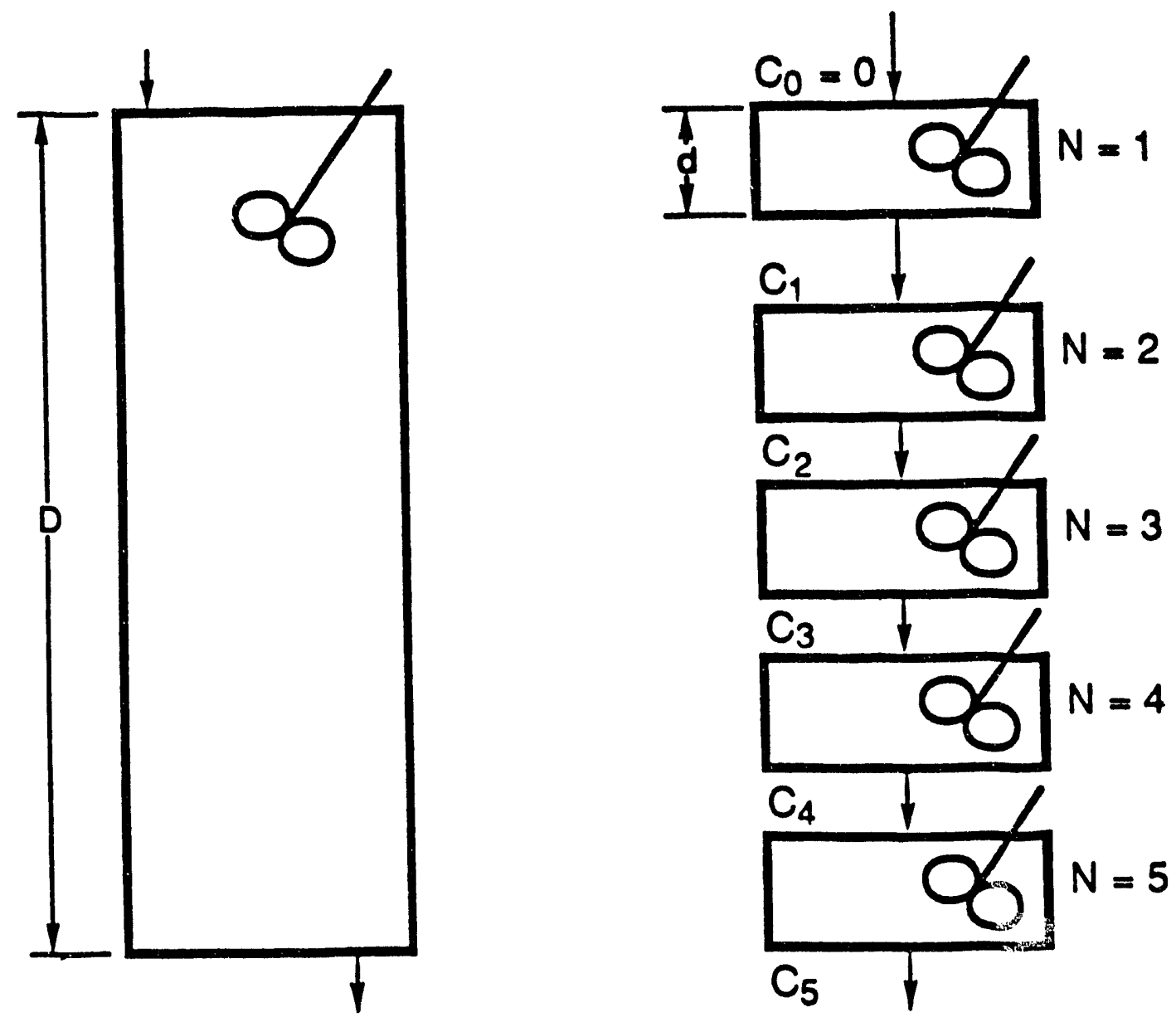

Figure A.1 Schematic representation of the mixing cell cascade approach. (a) a single mixing cell, (b) multiple mixing cells. [From Kozak, 1990] 
Equation (A.24) applies to each mixing cell. For the first cell, $C_{i-1}$ is set to zero. This is equivalent to assuming that no contaminant enters through the top of the facility. Therefore, we have a system of $\mathrm{N}$ coupled linear differential equations. This system of equations has been solved to provide an analytical solution for arbitrary wasteform sources, $q_{i}$, within each mixing cell.

The resulting expression for the concentration in the $\mathrm{N}^{\text {th }}$ mixing cell is:

$$
\begin{aligned}
C_{N}(t) & =e^{-\lambda t} e^{-\alpha N t} \sum_{n=0}^{N-1} \frac{(\alpha N t)^{n}}{n !} C_{N-n}(0) \\
& +\beta N e^{-\lambda t} e^{-\alpha N t} \sum_{n=0}^{N-1}(\alpha N)^{n} I_{n+1}
\end{aligned}
$$

where:

$$
I_{n+1}=\int_{0}^{1} d t_{1} \int_{0}^{t_{1}} d t_{2} \int_{0}^{t_{2}} \cdots \int_{0}^{t_{n}} d t_{n+1} \quad Q_{N-n}(t) e^{(\alpha N+\lambda) t}
$$

Equation (A.26) applies only if the parameter alpha is constant.

The first term in Eqn. (A.25) arises from the initial conditions, $\left(C_{n}(0)\right.$ is the concentration in the $\mathrm{n}^{\text {th }}$ cell at time $=0$ ). In most instances, the initial concentration in the solute is expected to be zero.

The integral in Eqn. (A.26) can be evaluated analytically for certain functional forms of the wasteform release rate term, $Q_{n}$. In particular, rinse release and dissolution release (constant release rate) with radioactive decay can be modeled as follows:

$$
\begin{aligned}
Q_{n}(t) & =C_{n}\left(\tau_{n, b}\right) \delta\left(t-\tau_{n, b}\right) \\
& +Q_{n}(0) e^{-\lambda}\left[H\left(t-\tau_{n, b}\right)-H\left(t-\tau_{n, \delta}\right)\right]
\end{aligned}
$$


where:

$$
C_{n}\left(\tau_{n, b}\right)=\frac{M_{r, n} e^{-\lambda \tau_{a, b}}}{\theta V_{n} R}
$$

where:

$\mathrm{M}_{\mathrm{r}, \mathrm{n}} \quad$ = the mass available for rinse release at $\mathrm{t}=0$;

$\tau_{n, b} \quad=$ time of total failure of the $n^{\text {th }}$ container;

$V_{n} \quad=$ the volume of the $n^{\text {th }}$ mixing cell (the volume of the entire disposal facility divided by the number of mixing cells, $\mathrm{N}$ );

$\delta\left(t-\tau_{\mathrm{n}, \mathrm{b}}\right) \quad=$ Dirac $\delta$ function;

$\mathrm{Q}_{\mathrm{n}}(0) \quad=$ release rate at time $=0$

$\mathrm{H}\left(\mathrm{t}-\tau_{\mathrm{n}, \mathrm{b}}\right) \quad=$ Heaviside function, defined as follows:

$$
\begin{array}{ll}
H\left(t-\tau_{n, b}\right)=0 & t<\tau_{n, b} \\
H\left(t-\tau_{n, b}\right)=1 & t>\tau_{n, b}
\end{array}
$$

$\tau_{\mathrm{n}, \mathrm{f}}=$ completion time for wasteform release, i.e., no further release is permitted after this time. This can be calculated by taking the inverse of the yearly fractional release rate and adding this value to the time of breach.

The first term represents the instantaneous surface wash-off that occurs immediately after container breach when water first contacts the wasteform. The second term models a constant release rate corrected for first order (radioactive) decay which starts immediately after container breach and finishes at the time the entire inventory is released.

If $Q_{n}(0)$ is used to model a dissolution controlled process, it would be estimated as the product of the dissolution velocity, surface area of the wasteform, and the concentration of contaminant within the wasteform as discussed in the wasteform release section of Chapter 2.

As currently written, the release rate term, $Q_{n}$, is not directly applicable to modeling the monotonically decreasing release rate representative of diffusion. Including a diffusion term is conceptually easy. However, because the analytical solution to diffusion release is an infinite series (see the diffusion release section in this Appendix), the multiple integrations required in Eqn. (A.29) are cumbersome. 
However, if the exponentially decaying release rate term is used to approximate release that is controlled by diffusion, $Q_{n}(0)$ would be estimated as the product of the diffusion coefficient, a geometric factor which involves the surface area, and the concentration in the wasteform.

Using the wasteform release rate term given in Eqn. (A.27) in the analytical solution, Eqn. (A.25), yields the following solution for the concentration in the last mixing cell:

$$
\begin{aligned}
& C_{N}(t)=e^{-\lambda t} e^{-\alpha N t} \sum_{n=0}^{N-1} C_{N-n}(0)(\alpha N t)^{n} / n ! \\
& +\sum_{n=0}^{N-1} C_{N-n}\left(\tau_{N-n, b}\right) H\left(t-\tau_{N-n, b}\right)\left(\alpha N\left(t-\tau_{N-n, b}\right)\right)^{n} \cdot e^{-(\alpha N+\lambda)\left(t-\tau_{N-\infty}\right)} / n ! \\
& +\frac{\beta}{\alpha} e^{-\lambda} \sum_{n=0}^{N-1} Q_{N-n}(0) H\left(t-\tau_{N-n, b}\right)\left(1-e^{-\alpha N\left(t-\tau_{N-m}\right)}\right) \\
& -\frac{\beta}{\alpha} e^{-\lambda} \sum_{n=1}^{N-1} Q_{N-n}(0) H\left(t-\tau_{N-n, b}\right) e^{-\alpha N\left(t-\tau_{N-a, b}\right)} \sum_{i=1}^{n} \frac{(\alpha N)^{i}\left(t-\tau_{N-n, b}\right)^{i}}{i !} \\
& -\frac{\beta}{\alpha} e^{-\lambda} \sum_{n=0}^{N-1} Q_{N-n}(0) H\left(t-\tau_{N-n \delta}\right)\left(1-e^{-\alpha N\left(t-\tau_{N-\alpha}\right)}\right) \\
& +\frac{\beta}{\alpha} e^{-\lambda s} \sum_{n=1}^{N-1} Q_{N-n}(0) H\left(t-\tau_{N-n f}\right) e^{-\alpha N\left(t-\tau_{N-2)}\right)} \sum_{i=1}^{n} \frac{\left(\alpha N\left(t-\tau_{N-n f}\right)\right)^{i}}{i !}
\end{aligned}
$$

The above expression for $\mathrm{C}_{\mathrm{n}}$ is quite general and permits each mixing cell to model a container with a unique time of breach, inventory, and release rate. The first term in Eqn. (A.29) represents the initial condition. The second term represents the concentration in solution due to surface rinse beginning immediately after breach. The remaining four terms represent the concentration of radioactivity in solution due to a wasteform that releases mass uniformly in time adjusted for radioactive decay.

A number of different situations can be adequately modaled with Eqn. (A.29) through proper selection of the input parameters. However, because of the restriction that the wasteform release rate be expressed either by surface rinse or an exponentially decaying rate, the above 
expression can not be used to investigate localized failures because they lead to time-dependent wasteform release rates (as the failure area grows, the release rate increases).

The mass flux out of the disposal facility is the concentration in the last cell multiplied by the Darcy velocity. The total mass flow rate is the mass flux multiplied by the area of the disposal facility.

$$
M_{\lambda}(t)=C_{n}(t) V_{d} A_{f}
$$

\section{Finite Difference Solution Procedure}

If the assumptions of the mixing cell cascade model (constant water flow, uniform retardation and moisture content, advection controlled transport, and only catastrophic container failures (e.g., no localized failures)) are not justified, the finite difference (FD) solution procedure is recommended.

The FD model uses a control volume approach when performing the mass balance. Figure A.2 presents a typical control volume discretization scheme. To define the system, the user supplies the number of positions at which the concentration is calculated, NNP, and the locations which are used to determine the control volumes, $X(I)$, where I ranges from 1 to NNP. In Figure A.2 the solid lines mark the locations defined through input. The dashed lines represent the control volumes. That is, the first concentration value is the average for the region contained by $X=X(1)$ and $X=1 / 2(X(2)-X(1))$; the second concentration value is for the region $X=1 / 2(X(2)-X(1))$ to $X=X(2)+1 / 2(X(3)-X(2))$; and so on. The average concentration can be viewed, although this is an approximation, as the concentration at the midpoint of the control volume. With this assumption, and for uniform spacing, the first concentration value represents the concentration at $(X(2)-X(1)) / 4$, the second represents the concentration at $\mathrm{X}(2)$, the third at $\mathrm{X}(3)$, etc. This pattern continues until the last control volume, where the concentration at the last computational point is approximately the concen'ration at the point given by $\mathrm{X}=\mathrm{X}(\mathrm{NNP})-1 / 4(\mathrm{X}(\mathrm{NNP})-\mathrm{X}(\mathrm{NNP}-1))$.

This indicates that the first and last concentration values are not boundary values. Boundary values are incorporated directly into the mass balance equation ven calculating the first and last concentration values. This procedure is described later in this Appendix.

Although it is not explicit in Fig. A.2, the size of the control volumes may vary with location. For non-uniform spacing, the midpoint of the control volume will not coincide with the input locations. For example, if $X(1)=0, X(2)=1$, and $X(3)=10$, control volume 1 will cover the range 0 to $1 / 2$, while control volume 2 is the region $1 / 2$ to 5.5 and has a midpoint of 3. This can become important when interpreting results. 


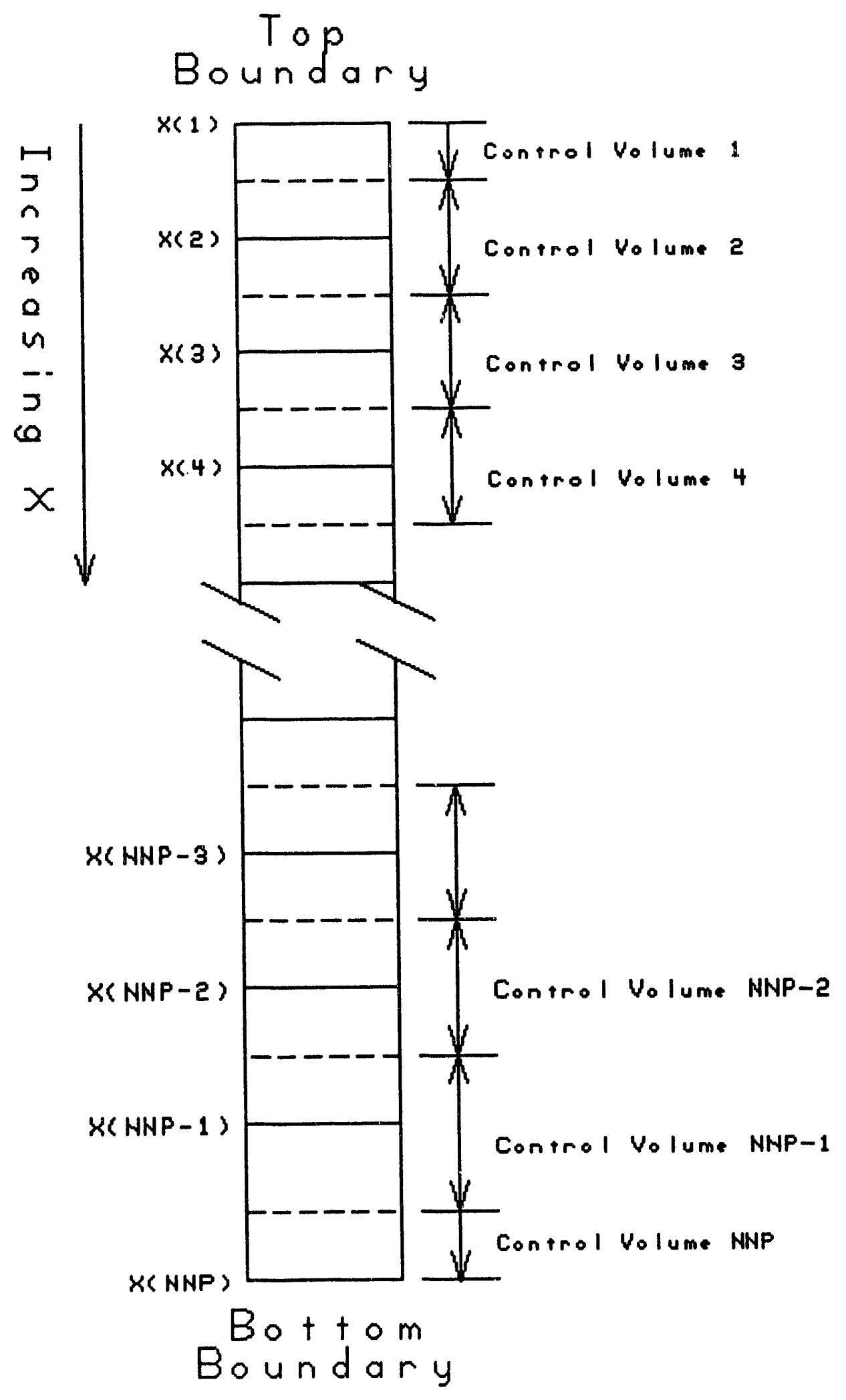

Figure A.2 Control volume discretization of a modeled region. 
In specifying containers, wasteform, and external source locations, the locations correspond to a control volume. That is, if a wasteform is placed in location 5 , it is in the region defined by control volume 5 .

The finite difference procedure begins with Eqn. (A.22). The second order derivative (the diffusion-dispersion term) is estimated using centered differences. The first order spatial derivative is approximated using upwind differencing. The time derivative term is approximated using a first order backward difference. Performing this, results in the following equation:

$$
\begin{aligned}
\frac{\left(\theta R C_{n}\right)^{i+1}}{\Delta t} & =\frac{\left(\theta R C_{n}\right)^{i}}{\Delta t} \\
& +C_{n+1}^{i+1}\left[\frac{(\theta D)_{n+1}^{i+1}}{\Delta X_{n} \Delta X_{n, a}}\right] \\
& -C_{n}^{i+1}\left[\frac{(\theta D)_{n}^{i+1}}{\Delta X_{n} \Delta X_{n, a}}+\frac{(\theta D)_{n}^{i+1}}{\Delta X_{n-1} \Delta X_{n, a}}+\frac{V_{D}^{i+1}}{\Delta X_{n-1}}+\lambda(\theta R)^{i+1}\right] \\
& +C_{n-1}^{i+1}\left[\frac{(\theta D)_{n-1}^{i+1}}{\Delta X_{n-1} \Delta X_{n, a}}+\frac{V_{D}^{i+1}}{\Delta X_{n-1}}\right] \\
& +q_{n}^{i+1}
\end{aligned}
$$

where $\Delta X_{n, a}=\frac{1}{2}\left(\Delta X_{n}+\Delta X_{n-1}\right)$

where the superscript $i$ refers to the time level of the calculation and the subscript $\mathrm{n}$ refers to the spatial location of the calculation. Except for the time-derivative term, all concentrations are evaluated at the new time level, $\mathrm{i}+1$. This is known as the implicit solution procedure and insures that the numerical solution is stable, i.e. numerical errors that occur in solving the equation are damped. 
Equation (A.31) applies for each control volume in the interior ( $\mathrm{n}$ not equal to 1 or $\mathrm{N}$ ) of the domain being simulated. Equation (A.31) illustrates that the concentration in control volume $\mathrm{n}$ is a function of its two nearest neighbors. The system of equations which accounts for the concentration at each point can be rearranged such that all unknown variables at the new time level are on the left hand side of the equation and all known variables (the release rate and concentrations at the old time level) are on the right hand side. Performing this arrangement and using matrix notation yields:

$$
\overline{A C}(i+1)=\bar{S}
$$

where $\mathrm{A}$ is a tridiagonal matrix comprised of the terms that multiply the concentrations in each control volume at time level $i+1, C$ is a vector that represents the concentration in each control volume at time level $i+1$, and $S$ is a source term comprised of the release rate from the wasteform over the time step plus the concentration at time level $i$. The system of equations represented by Eqn. (A.32) can be solved quite easily using standard numerical techniques.

\section{Boundary Conditions}

Special consideration is given to the first and last control volume. These control volumes are modified to reflect the boundary conditions imposed on the problem. Typical boundary conditions are tihat the concentration or the flux is specified as a function of time. The boundary condition applied to the top of the disposal facility could be zero mass flux entering the facility. At the bottom of the facility, the concentration or the flux may be specified. Requiring the concentration at the bottom of the facility to be zero would lead to the highest mass flux out of the facility. Similarly, requiring zero mass flux out would lead to the highest solution concentration. In addition, the user can specify the advective and the diffusive/dispersive flux at the boundary. Time dependence of the boundary conditions is modeled through an input table of values versus time.

The mass balance equation for any control volume of width $\Delta \mathrm{X}$ is:

$$
\frac{\partial(\theta R C)}{\partial t}=-\left[\frac{J_{\text {out }}-J_{\text {in }}}{\Delta X}\right]-\lambda \theta R C+q
$$

where $\mathrm{J}_{\text {out }}$ is the mass flux at the downstream boundary (largest value of $\mathrm{X}$ ) and $\mathrm{J}_{\mathrm{in}}$ is the mass flux at the upstream boundary. 
For interior control volumes, the mass fluxes are evaluated using Eqn. (A.21). At the boundary control volumes, the boundary mass flux is incorporated directly into the equation based on the choice of boundary condition.

\section{First Control Volume}

For the top boundary, e.g., the first control volume (Fig. A.2), the finite difference mass balance becomes:

$$
R_{1}\left[\frac{C_{1}^{j+1}-C_{1}^{i}}{\Delta t}\right]=-\left[\frac{J_{1}-J_{b}}{\Delta X / 2}\right]-\lambda R_{1} C_{1}+q_{1}
$$

where $\Delta \mathrm{X}=\mathrm{X}(2)-\mathrm{X}(1)$, the subscripts 1 refer to control volume 1 , and the superscript $\mathrm{i}$ refers to the time level of the calculation.

The boundary flux, $\mathrm{J}_{\mathrm{b}}$, is:

$$
J_{b}=-\theta D_{1}\left[\frac{C_{1}-C_{b}}{\Delta X_{1} / 4}\right]+V_{d} C_{b}
$$

This can be specialized for the four boundary conditions (BC) as follows:

BC 1: Specified Concentration

The $\mathrm{BC}$ is:

$$
C_{b}=g(t)
$$

where $g(t)$ is specified in an input table. 
In this case:

$$
J_{b}=-\theta D_{1}\left[\frac{C_{1}-g(t)}{\Delta X_{1} / 4}\right]+V_{d} g(t)
$$

BC 2: Specified Total Flux

This $\mathrm{BC}$ is:

$$
J_{b}(t)=g(t)
$$

BC 3: Specified Advective Flux

This $\mathrm{BC}$ is:

$$
V_{d} C_{b}=g(t)
$$

This is mathematically equivalent to specifying the concentration at the boundary.

In this case:

$$
J_{b}=-\theta D_{1}\left[\frac{C_{1}-g / V_{d}}{\Delta X / 4}\right]+g(t)
$$

BC 4: Specified Diffusive/Dispersive Flux

This $\mathrm{BC}$ is:

$$
-\theta D \frac{\partial C}{\partial x}=z(t)
$$


Here,

$$
J_{b}=g(t)+V_{d}\left[C_{1}+\frac{4}{\theta D \Delta X} g(t)\right]
$$

In this case, the boundary flux depends on the concentration in the first volume element, $C_{1}$. This reflects the link between the concentration at the boundary and in the first element due to diffusion/dispersion.

Depending on the choice of the BC, one of equations (A.37), (A.38), (A.40), or (A.42) is used directly in Eqn. (A.34) to generate the appropriate finite difference representation of the first control volume.

\section{Equations for the Last Control Volume}

For the bottom boundary, e.g., the last control volume, (Fig. A.2), the finite difference mass balance becomes:

$$
R_{N}\left[\frac{C_{N}^{i+1}-C_{N}^{i}}{\Delta t}\right]=-\left[\frac{J_{b}-J_{N}}{\Delta X_{N} / 2}\right]-\lambda R_{N} C_{N}+q_{N}
$$

where $\Delta \mathrm{X}_{\mathrm{N}}=\mathrm{X}(\mathrm{NNP})-\mathrm{X}(\mathrm{NNP}-1)$ in Fig. A.2, the subscript $\mathrm{N}$ refers to the last control volume, and $\mathrm{i}$ refers to the time level.

The boundary flux, $\mathrm{J}_{\mathrm{b}}$, is:

$$
J_{b}=-\theta D_{N}\left[\frac{C_{b}-C_{N}}{\Delta x_{N} / 4}\right]+V_{d} C_{N}
$$

Here the advective flux is independent of the boundary condition. This is consistent with the upwind finite differencing procedure used to generate the previous control volume mass balance equations. follows:

The four choices for boundary condition are used to calculate the boundary flux as 
BC 1: Specified Concentration

The $\mathrm{BC}$ is:

$$
C_{b}=g(t)
$$

where $g(t)$ is specified through tabular input.

In this case:

$$
J_{b}=-\theta D\left[\frac{g(t)-C_{N}}{\Delta x_{N} / 4}\right]+V_{d} C_{N}
$$

BC 2: Specified Total Flux

The $\mathrm{BC}$ is:

$$
J_{b}=g(t)
$$

BC 3: Specified Advective Flux

The $\mathrm{BC}$ is:

$$
V_{d} C_{b}=g(t)
$$

Here,

$$
J_{b}=-\theta D\left[\frac{g(t) / V_{d}-C_{N}}{\Delta x_{N} / 4}\right]+V_{d} C_{N}
$$


Again, this is mathematically equivalent to specifying the concentration. In general, at the last boundary unless the advective flux is zero, it will be difficult to specify a priori. Further, it may lead to an inconsistency in the interpretation of the boundary advective flux. In the mass balance equation, Eqn. (A.43), the advective flux is $V \mathrm{C}_{\mathrm{N}}$. However, the boundary condition advective flux is given as $g(t)$.

BC 4: Specified Diffusive/Dispersive Flux

The $\mathrm{BC}$ is:

$$
-\theta D \frac{\partial C}{\partial x}=g(t)
$$

In this case,

$$
J_{b}=g(t)+V_{d} C_{N}
$$

Depending on the choice of boundary condition, one of equations (A.46), (A.47), (A.49), or (A.51) is used in Eqn. (A.44) to generate the appropriate finite difference representation of the last control volume. 


\section{REFERENCES}

[Kozak, 1990]

M.W. Kozak, et. al., Background Information for the Development of a Low-Level Waste Performance Assessment Methodology: Computer Code Implementation and Assessment," NUREG/CR-5453, SAND89-2509, Volume 5, Sandia National Laboratories, 1990.

[Pescatore, 1991]

C. Pescatore, "Leach Rate Expressions for Performance Assessment of Solidified, Low-Level Radioactive Waste," accepted for publication by the Journal of Waste Management, also available as an informal report, WM-3276-12, Brookhaven National Laboratory, December, 1990. 


\section{APPENDIX B}

\section{FLOW CHART FOR THE DUST CODE}

The DUST code contains 17 subroutines as depicted in Figure B.1. This appendix provides a brief description of each subroutine and the organization of the computer code.

The main program is called DUST and it contains the dimension statements and common blocks for all of the primary variables. The DUST program calls the subroutine GM.

GM, general manager, is the shell that controls all program operations. Initialization of key variables, calling the input routine, DATAIN, controlling the choice of transport model (Finite Difference (FD) or Multi-Cell Mixing Cascade (MCMC)), determining when output is required, and advancing the calculation in time are all performed by $\mathrm{GM}$.

The subroutine DATAIN reads in all input parameters. As discussed in chapter 7, the input deck is divided into 10 data sets. Each data set contains the parameters required by the models used in the code, that is, one data set contains all the wasteform release parameters, etc. To facilitate the need for inputting a large number of parameters DATAIN relies on five auxiliary input subroutines: INPTR, INPTR2, INPTI, READR, and READN.

The INPTR subroutine is used to read in an array of real numbers. The DUST code reads the arrays: TIMOUT (output times for the MCMC model or time step change times for the FD model); TFAIL (time of total container failure); and WTINIT (initial mass in each wasteform) using INPTR.

The INPTR2 subroutine is used to read in two-dimensional arrays of real numbers which form the interpolation tables for boundary conditions, darcy velocity, and external sources. These arrays include: TBC (time of the boundary condition for each boundary); FBC (value of the boundary condition at the specified times for each boundary); TVDAR (time of the darcy velocity data points); FVDAR (value of the darcy velocity at the specified times); TSOSF (time of the source for each source profile); and SOSF (value of the source at the specified time for each profile).

The INPTI subroutine is used to read in arrays of integer numbers. The arrays include: NTRCEC (nodes for concentration traces); NTRCEF (nodes for flux traces); IPIT (pitting corrosion flag used in the FD model); NELCON (identifies the nodes with a waste container); ICTYPE (flag defining the container type in the FD model, that is, the DUST code permits several container types each with a unique set of localized failure parameters, this flag connects each container with a set of failure parameters), IDIFF (geometry flag for calculating diffusioncontrolled releases in the FD model); IWTYPE (flag defining the wasteform type); LSRC (location of external sources); and ISTYPE (flag defining the type of source). 
The READR subroutine is used to generate a sequence of values for selected real arrays. READR is used to define the node locations $(\mathrm{X})$ in the FD model, the initial conditions for both transport models, and the moisture content at each location in the FD model.

The READN subroutine is used to generate a sequence of values for the integer array IMAT which contains the material properties index when more than one material is used (FD model only).

The exact format and parameters required for each of these input routines is described in detail in Chapter 7 of the main body of this report.

Upon completion of the input routine program control is returned to subroutine GM. GM advances the time, calls the subroutine INTERP to calculate the value for external sources at the new time and checks to determine if the FD or MCMC transport model has been selected.

If the FD model is selected the subroutine BREACH is called. BREACH calculates localized failure rates and breached area as well as checks if the time to total failure has been exceeded. After failure occurs, material is released from the wasteform as calculated in subroutine LEACH. This release rate is fed into subroutine FDASSM which assembles the finite difference matrix used to calculate the migration of the radionuclides. FDASSM calls subroutine BOUND which incorporates the specified boundary conditions directly into the matrix. Upon completion of the generation of the matrix, the subroutine FDSLV, is used to solve the tridiagonal matrix equation giving the concentration at the new time level at each location.

If the MCMC model is selected, the subroutine MIXBATH is called. MIXBATH checks if the time to failure of each container has been exceeded. If failure occurs, MIXBATH calculates the release rate from these containers and solves the analytical expression for transport of radionuclides through the facility.

After completion of the calculation of the concentrations, checks are made to determine if output is requested at the current time step. Flux and concentration traces are written to separate files within subroutine TRACE. The primary output file is written by subroutine PRINTT.

After output has been completed, subroutine GM advances the time variable and the procedure is repeated until the maximum number of time steps or maximum problem simulation time are exceeded.

Although subroutines FLUX and INTERP are located between subroutines TRACE and PRINTT in the flow chart, these subroutines are called several times during the calculation procedure. In particular, subroutine FLUX which calculates the total flux at every point is called prior to entering TRACE or PRINTT. Subroutine INTERP, which interpolates from a table, is used to calculate external sources, darcy velocity, and boundary conditions at the current simulation time. 


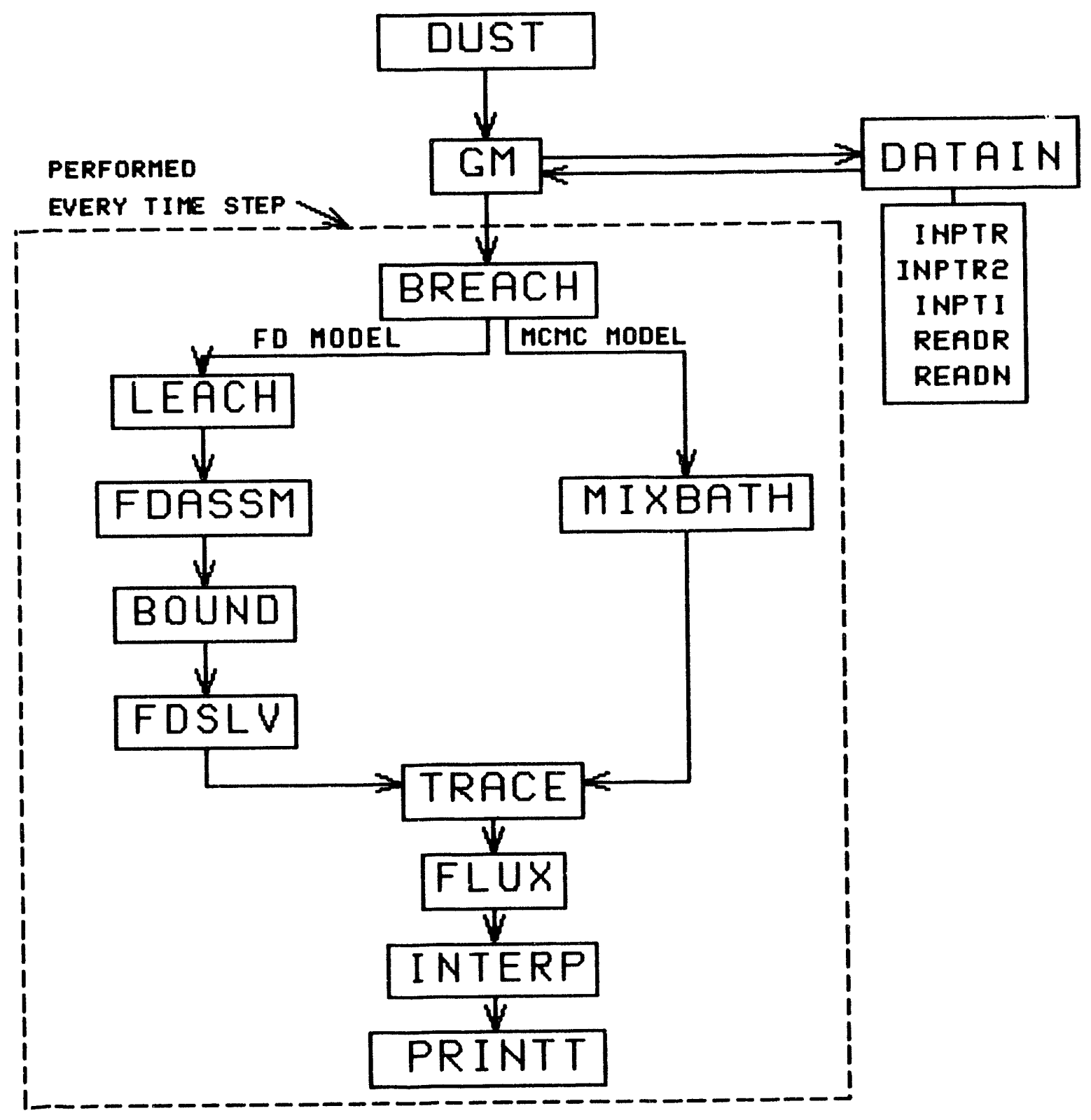

Figure B.1 DUST" Subroutine Flow Chart. 


\section{APPENDIX C}

\section{DATA REQUIREMENTS FOR THE PITTING MODEL IN SUBROUTINE BREACH}

The container degradation models require information on the thickness and corrosion rates of the container. For 55 gallon carbon steel drums, typical thicknesses range from 50 - 60 mils, $0.127-0.152 \mathrm{~cm}$.

For the pitting model, five parameters are required: the rate constants $k$ and $n$, the area of the container, the area scaling factor, and the number of penetrating pits in the container.

The values of $\mathbf{k}$ and $\mathrm{n}$ for carbon steel have been determined from the National Bureau of Standards, NBS, data on corrosion in soils. $k$ was found to range from $0.03-0.15 \mathrm{~cm} / \mathrm{yr}^{\mathrm{n}}$ with a mean value of $0.074 \mathrm{~cm} / \mathrm{yr}^{n}$. $\mathrm{n}$ was found to range from $0-0.92$ with a mean value of 0.39 . A list of values for $k$ and $n$ in 47 different soils can be found in Romanoff [Romanoff, 1957]. This list has been ordered by soil aeration and reproduced in the previous Source Term topical report [Sullivan, 1988]. $\mathrm{k}$ was found to be correlated with soil $\mathrm{pH}$. $\mathrm{n}$ was found to be strongly correlated with the degree of soil aeration. The poorer the aeration, the higher the value of $n$.

The data base for stainless steels was insufficient to calculate the pitting parameters $k$ and n. After 14 years, often there was no measurable pitting.

The area of a 55 gallon drum is $2.1 \mathrm{E} 4 \mathrm{~cm}^{2}$. For other waste forms, this model requires the surface area in $\mathrm{cm}^{2}$.

The area scaling factor has been measured by Logan [Logan, 1939] for wrought iron pipes buried in 47 different soils. Values ranged from $0.08-0.32$ with a mean value of 0.149 and a standard deviation of 0.042 . Table C-1 presents this data grouped by degree of soil aeration. The scaling factor did not exhibit a significant dependence on degree of aeration.

The number of penetrating pits has been estimated as 0.05 per $\mathrm{cm}^{2}$. DUST requires the number of penetrating pits per container. Thus, for a 55 gallon drum with a surface area of $2.1 \mathrm{E} 4 \mathrm{~cm}^{2}$, this implies 1050 penetrating pits.

The general corrosion rate for carbon steels and stainless steels has also been obtained from the NBS corrosion data in soils [Romanoff, 1957]. For carbon steels, these rates are presented in Table C-2 and ranged from $2.7 \mathrm{E}-11-6 \mathrm{E}-10 \mathrm{~cm} / \mathrm{s}$ in a sample of 15 different soils. The mean value was $1.8 \mathrm{E}-10 \mathrm{~cm} / \mathrm{s}$ and the standard deviation was $1.5 \mathrm{E}-10 \mathrm{~cm} / \mathrm{s}$. These values are the average rate for the duration of the experiment, 8 - 14 years. It was often noted that corrosion proceeded at a high initial rate and slowed down in time. 
In the same soils, for 304 stainless steel, the average corrosion rate was $8.9 \mathrm{E}-14 \mathrm{~cm} / \mathrm{s}$ with a standard deviation of $1.6 \mathrm{E}-13 \mathrm{~cm} / \mathrm{s}$. The range of measured corrosion rates was $5.3 \mathrm{E}-13$ $3.5 \mathrm{E}-15 \mathrm{~cm} / \mathrm{s}$. For 316 stainless steel, the average corrosion rate was $4.1 \mathrm{E}-14 \mathrm{~cm} / \mathrm{s}$ with a standard deviation of $4.9 \mathrm{E}-14 \mathrm{~cm} / \mathrm{s}$. The range of measured corrosion rates was $1.8 \mathrm{E}-13$ $8.9 \mathrm{E}-16 \mathrm{~cm} / \mathrm{s}$. Table C-3 presents the corrosion rates in the various soils for these two metals. Sensitized steels corrode at a rate of 1 to 2 orders of magnitude faster [Gerhold, 1981].

\section{REFERENCES}

[Gerhold, 1981]

Gerhold, W.F., E. Escalante, and B.T. Sanderson, "The Corrosion Behavior of Selected Stainless Steels in Soil Environments, " NBSIR 81-2228, National Bureau of Standards, 1981.

[Logan, 1939]

Logan, K.H., "Engineering Significance of National Bureau of Standards Soil-Corrosion Data," Journal of Research of the National Bureau of Standards, Vol. 22, January 1939.

[Romanoff, 1957]

Romanoff, M., "Underground Corrosion," National Bureau of Standards Circular 579, 1957.

[Sullivan, 1988]

Sullivan, T.M., C.R. Kempf, C.J. Suen, and S.F. Mughabghab, "Low-Level Radioactive Waste Source Term Model Development and Testing," NUREG/CR-5204, BNL-NUREG-52160, Brookhaven National Laboratory, 1988. 
Table $c-1$

Values for the area scaling factor on wrought iron samples [From Logan, 1939].

\begin{tabular}{|c|c|c|c|}
\hline Soil & Aeration & Area Scaling Factor & Comments \\
\hline $\begin{array}{r}3 \\
6 \\
11 \\
12 \\
13 \\
18 \\
22 \\
24 \\
31 \\
32 \\
35 \\
36 \\
38 \\
44 \\
46\end{array}$ & $\begin{array}{l}\text { Good } \\
\text { Good } \\
\text { Good } \\
\text { Good } \\
\text { Good } \\
\text { Good } \\
\text { Good } \\
\text { Good } \\
\text { Good } \\
\text { Good } \\
\text { Good } \\
\text { Good } \\
\text { Good } \\
\text { Good } \\
\text { Good }\end{array}$ & $\begin{array}{l}0.144 \pm 0.024 \\
0.144 \pm 0.028 \\
0.140 \pm 0.021 \\
0.140 \pm 0.031 \\
0.193 \pm 0.015 \\
0.111 \pm 0.018 \\
0.082 \pm 0.009 \\
0.172 \pm 0.035 \\
0.172 \pm 0.027 \\
0.165 \pm 0.039 \\
0.316 \pm 0.007 \\
0.097 \pm 0.014 \\
0.153 \pm 0.028 \\
0.148 \pm 0.038 \\
0.186 \pm 0.011\end{array}$ & $\begin{array}{l} \\
\\
\text { Average for Good } \\
0.158 \pm 0.054 \\
\text { Not including soil } 35 \\
\text { Average is } \\
0.146 \pm 0.032\end{array}$ \\
\hline $\begin{array}{l}9 \\
10 \\
14 \\
16 \\
19 \\
23 \\
25 \\
26 \\
37 \\
41 \\
42\end{array}$ & $\begin{array}{l}\text { Fair } \\
\text { Fair } \\
\text { Fair } \\
\text { Fair } \\
\text { Fair } \\
\text { Fair } \\
\text { Fair } \\
\text { Fair } \\
\text { Fair } \\
\text { Fair } \\
\text { Fair }\end{array}$ & $\begin{array}{l}0.172 \pm 0.028 \\
0.184 \pm 0.027 \\
0.155 \pm 0.033 \\
0.118 \pm 0.017 \\
0.103 \pm 0.010 \\
0.139 \pm 0.044 \\
0.088 \pm 0.008 \\
0.120 \pm 0.028 \\
0.191 \pm 0.017 \\
0.098 \pm 0.009 \\
0.095 \pm 0.009\end{array}$ & $\begin{array}{l}\text { Average for Fair } \\
0.133 \pm 0.037\end{array}$ \\
\hline $\begin{array}{r}1 \\
2 \\
4 \\
5 \\
7 \\
8 \\
15 \\
17 \\
20 \\
21 \\
27 \\
30 \\
34 \\
39 \\
40 \\
45 \\
47\end{array}$ & $\begin{array}{l}\text { Poor } \\
\text { Poor } \\
\text { Poor } \\
\text { Poor } \\
\text { Poor } \\
\text { Poor } \\
\text { Poor } \\
\text { Poor } \\
\text { Poor } \\
\text { Poor } \\
\text { Poor } \\
\text { Poor } \\
\text { Poor } \\
\text { Poor } \\
\text { Poor } \\
\text { Poor } \\
\text { Poor }\end{array}$ & $\begin{array}{l}0.149 \pm 0.021 \\
0.131 \pm 0.030 \\
0.160 \pm 0.020 \\
0.144 \pm 0.030 \\
0.116 \pm 0.009 \\
0.133 \pm 0.009 \\
0.100 \pm 0.015 \\
0.125 \pm 0.038 \\
0.188 \pm 0.038 \\
0.129 \pm 0.012 \\
0.176 \pm 0.037 \\
0.123 \pm 0.033 \\
0.157 \pm 0.012 \\
0.150 \pm 0.015 \\
0.144 \pm 0.019 \\
0.142 \pm 0.025 \\
0.237 \pm 0.030\end{array}$ & $\begin{array}{l}\text { Average for Poor } \\
0.147 \pm 0.032\end{array}$ \\
\hline
\end{tabular}


Table C-1 (cont.)

\begin{tabular}{llll} 
Soil & Aeration & Area Scaling Factor & \\
\cline { 3 - 4 } 28 & Very Poor & $0.106 \pm 0.045$ & \\
29 & Very Poor & $0.176 \pm 0.043$ & \\
33 & Very Poor & $0.168 \pm 0.028$ & Average for Very Poor \\
43 & Very Poor & $0.195 \pm 0.023$ & $0.161 \pm 0.039$
\end{tabular}

Soil properties are described in detail in Romanoff [Romanoff, 1957].

Table C-2

General Corrosion rates of Carbon Steels in soils

[Adapted from Table 15 in Romanoff, 1957].

\begin{tabular}{|c|c|c|}
\hline Soil & Time (yrs) & $\begin{array}{l}\text { Corrosion Rate } \\
(\mathrm{cm} / \mathrm{s}) \star 10^{10}\end{array}$ \\
\hline 51 & 14.3 & 1.8 \\
\hline 53 & 12.7 & 0.38 \\
\hline 53 & 14.3 & 0.38 \\
\hline 55 & 12.6 & 0.32 \\
\hline 55 & 14.3 & 0.27 \\
\hline 56 & 7.5 & 4.4 \\
\hline 56 & 8.9 & 3.9 \\
\hline 58 & 12.7 & 1.7 \\
\hline 58 & 14.3 & 2.2 \\
\hline 59 & 12.7 & 1.1 \\
\hline 59 & 14.2 & 0.32 \\
\hline 60 & 12.7 & 2.1 \\
\hline $\begin{array}{l}60 \\
61\end{array}$ & $\begin{array}{l}14.3 \\
12.7\end{array}$ & $\begin{array}{l}2.5 \\
0.73\end{array}$ \\
\hline 61 & 14.4 & 0.86 \\
\hline 62 & 12.7 & 0.67 \\
\hline 62 & 14.3 & 0.67 \\
\hline 63 & 12.6 & 1.8 \\
\hline 63 & 14.4 & 0.83 \\
\hline 64 & 7.3 & 6.0 \\
\hline 64 & 12.3 & 1.8 \\
\hline $\begin{array}{l}65 \\
65\end{array}$ & 12.7 & 0.70 \\
\hline $\begin{array}{l}65 \\
66\end{array}$ & $\begin{array}{l}14.2 \\
9.2\end{array}$ & $\begin{array}{l}1.1 \\
2.5\end{array}$ \\
\hline 66 & 12.7 & 2.0 \\
\hline 67 & 7.3 & 5.4 \\
\hline 67 & 11.1 & 4.1 \\
\hline 70 & 12.8 & 2.1 \\
\hline
\end{tabular}

Comments

For a complete description of the soils see Table 6 in Romanoff, 1957.

Several soils had different samples removed at different times.

Average Corrosion rate:

$1.810^{-10} \pm 1.610^{-10} \mathrm{~cm} / \mathrm{s}$ or

$6.410^{-3} \pm 510^{-3} \mathrm{~cm} / \mathrm{yr}$.

Range of Corrosion rates:

$(0.27-6) 10^{-10} \mathrm{~cm} / \mathrm{s}$ or

$(0.85-19) 10^{-3} \mathrm{~cm} / \mathrm{yr}$. 
Table $\quad c-3$

General Corrosion Rate of Stainless jueels in Soil Environments for 14 years [Adapted 1.om Table 25 in Romanoff, 1957].

\begin{tabular}{|c|c|c|}
\hline Soil & $\begin{array}{l}304 \text { SS } \\
\text { Corrosion Rate } \\
(\mathrm{cm} / \mathrm{s}) * 10^{14} \\
\end{array}$ & $\begin{array}{l}316 \text { SS } \\
\text { Corrosion Rate } \\
(\mathrm{cm} / \mathrm{s}) * 10^{14}\end{array}$ \\
\hline $\begin{array}{l}51 \\
53 \\
55 \\
56 \\
58 \\
59 \\
60 \\
61 \\
62 \\
63 \\
64 \\
65 \\
66 \\
67 \\
70\end{array}$ & $\begin{array}{c}27 . \\
0.8 \\
18 . \\
1.8 \\
0.62 \\
0.53 \\
0.36 \\
0.35 \\
53.1 \\
2.7 \\
8.9 \\
0.8 \\
0.71\end{array}$ & $\begin{array}{c}8.0 \\
18.0 \\
3.6 \\
4.4 \\
0.71 \\
1.8 \\
5.3 \\
3.6 \\
0.18 \\
0.89 \\
1.8 \\
0.36 \\
\\
8.9 \\
0.09\end{array}$ \\
\hline
\end{tabular}

Comments

For the physical and

ciemical characteristics of the soils see Table 6 in Romanoff, 1957.

Average Values:

304 SS $-(8.9 \pm 16) 10^{-14}$

316 SS $-(4.1 \pm 4.9) 10^{-14}$

Range of Values:

304 SS - $(0.35-53) 10^{-14}$

316 SS - $(0.09-18) 10^{-14}$

0.09 

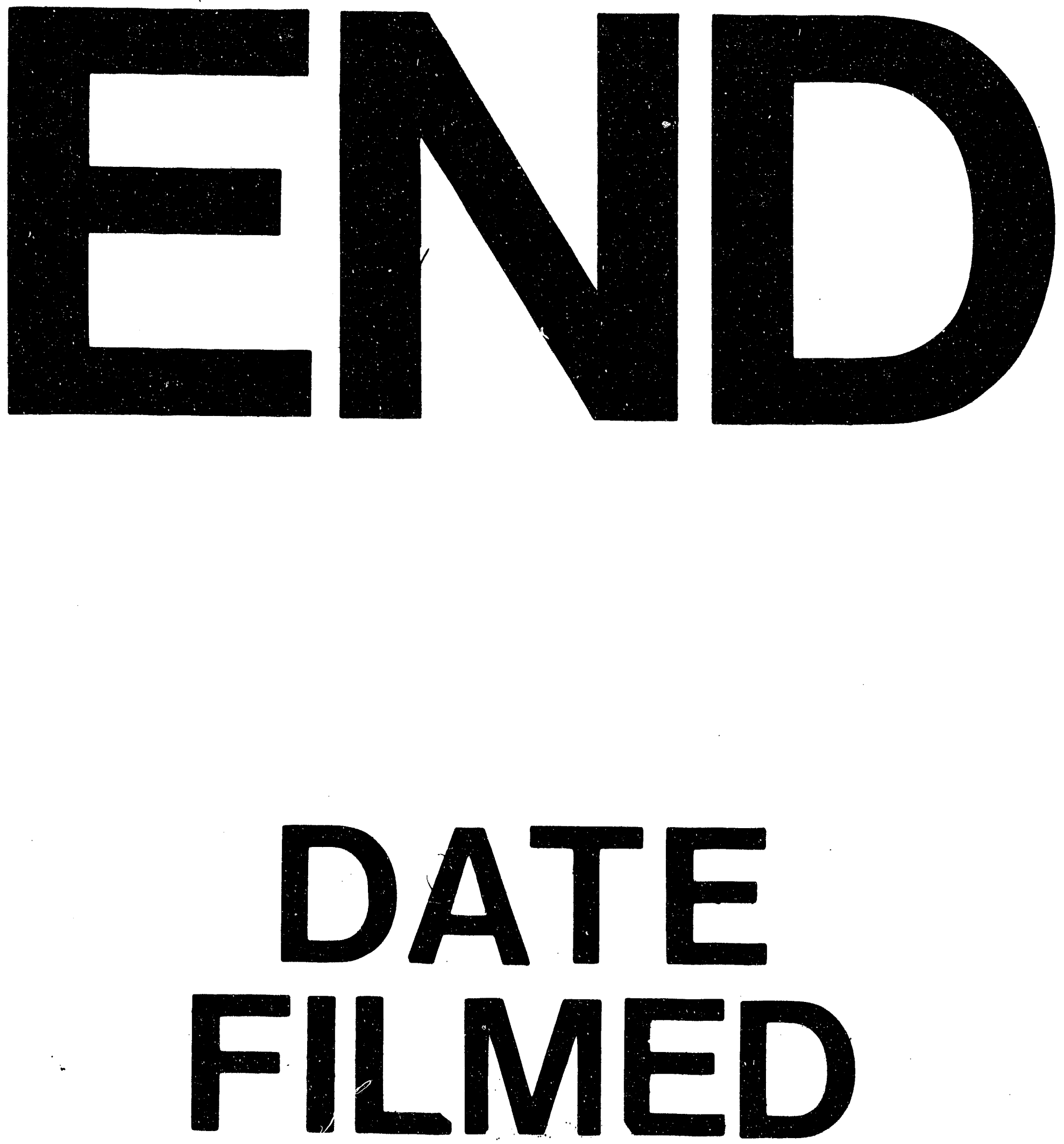

,

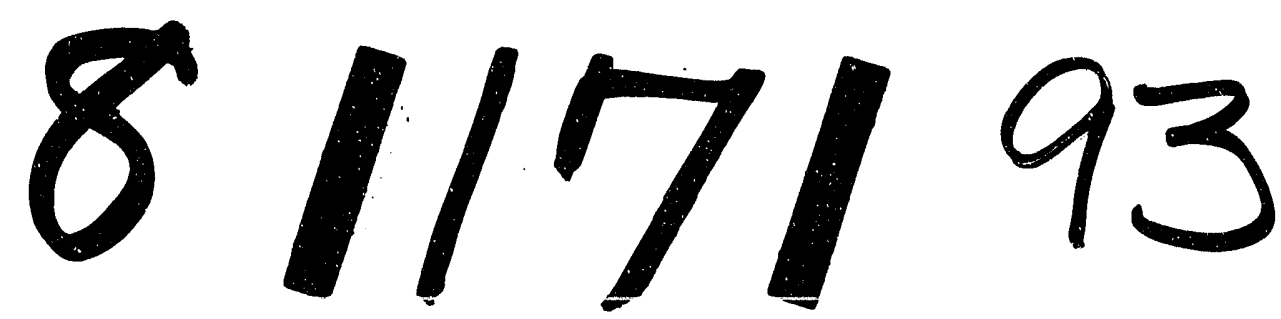


